PREDICTING SURFACE ROUGHNESS AND DELAMINATION WHEN ABRASIVE

WATERJET MACHINING FIBER REINFORCED POLYMER COMPOSITES

\author{
by
}

Jeff Schwartzentruber

MASc, Mechanical Engineering, Ryerson University, Canada, 2013

BEng, Aerospace Engineering, Ryerson University, Canada, 2011

\author{
A dissertation \\ presented to Ryerson University \\ in partial fulfillment of the \\ requirement for the degree of \\ Doctor of Philosophy \\ in the program of
}

Mechanical and Industrial Engineering

Toronto, Ontario, Canada, 2018

(C) Jeff Schwartzentruber, 2018 


\section{AUTHOR'S DECLARATION}

I hereby declare that I am the sole author of this dissertation. This is a true copy of the dissertation, including any required final revisions, as accepted by my examiners.

I authorize Ryerson University to lend this dissertation to other institutions or individuals for the purpose of scholarly research.

I further authorize Ryerson University to reproduce this dissertation by photocopying or by other means, in total or in part, at the request of other institutions or individuals for the purpose of scholarly research.

I understand that my dissertation may be made electronically available to the public. 


\title{
PREDICTING SURFACE ROUGHNESS AND DELAMINATION WHEN ABRASIVE WATERJET MACHINING FIBER REINFORCED POLYMER COMPOSITES
}

Doctor of Philosophy, 2018, Jeff Schwartzentruber, Mechanical and Industrial Engineering, Ryerson University

\begin{abstract}
The machining of composite materials is difficult because of their non-homogenous structure and their constituents commonly possess a high resistance to cutting. Abrasive waterjet machining (AWJM) is more attractive for composite substrates than conventional machining techniques because of its ability to rapidly machine a wide variety of materials with low reactionary forces on the workpiece, and without creating a heat-affected zone. However, AWJM is prone to producing variable surface roughness and delamination. This dissertation aimed to model these surface roughness and delamination mechanisms.
\end{abstract}

The thesis presents $2 \mathrm{D}$ and 3D roughness models capable of predicting the surface roughness during abrasive waterjet (AWJ) trimming of composite substrates. The models were able to predict the measured surface roughness with an average error of $10 \%$ and $16 \%$, for the 2D and 3D models, respectively.

The thesis also presents experimental and numerical results characterizing the delamination when AWJ piercing and cutting a carbon-fiber/epoxy laminate. Fluid-structure interaction (FSI) models created to simulate the piercing process showed that interlaminar delamination was due to the hydraulic shock ('water hammer') associated with liquid jet impact. As expected, increased pressure and nozzle size resulted in ply debonding, and was experimentally verified using 3D x-ray micro-tomography. The composite anisotropy was found to produce an asymmetric shock loading along the liquid-solid interface, which contributed to the asymmetric delamination.

The FSI model showed that delamination when cutting carbon-fiber/epoxy depended primarily on the normal interlaminar stress, with relatively large damage zones occurring ahead of the cutting front. This trend was also observed in x-ray micro-tomographs of an AWJ cut. The amount of delamination across different process parameters was also measured using a moisture uptake methodology, and showed that increase traverse speed, increased nozzle size, and 
decreased abrasive flow rate, increased delamination. Prediction and characterization of surface roughness and delamination when AWJM will allow further improvement of cut-surface finish and structural integrity of composite materials, respectively. 


\section{Acknowledgements}

I would like to thank:

$>$ Dr. Marcello Papini and Dr. Jan K. Spelt for providing me with the opportunity of joining their research team and their persistent support and advice in all steps of my $\mathrm{PhD}$.

$>$ The financial support of the Natural Sciences and Engineering Research Council of Canada (NSERC) and the Canada Research Chairs Program.

$>$ John Ingram and lab technicians at MAGNA Interiors (Concord, ON, CA) for providing the materials and technical support.

$>$ Dr. Chidambaram Narayanan for his advice and use of his abrasive waterjet energy model.

$>$ Ryerson University for the fantastic facilities and equipment provided for this research.

$>$ Chao Ma, Joseph Amankrah, Alan Machin, Grace He, and Qiang Li, from Ryerson University, for their technical support.

$>$ Dr. Mark Kortschot and Shiang Law from the University of Toronto for use of their 3D micro-tomography scanner.

My wife Dr. Kristy Schwartzentruber (Timmons) for her continual encouragement and support. 


\section{Table of contents}

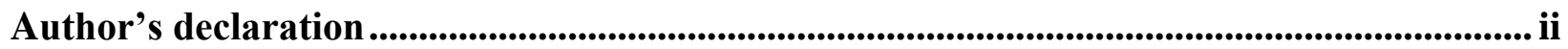

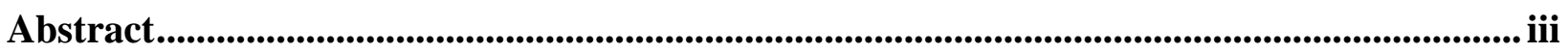

Acknowledgements .............................................................................................................................................. V

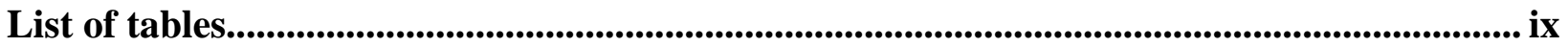

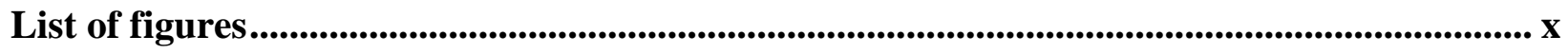

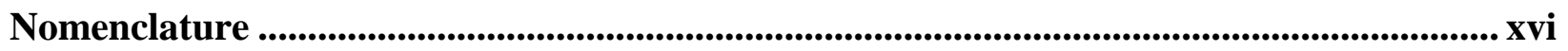

Chapter 1 Introduction..................................................................................................................................... 1

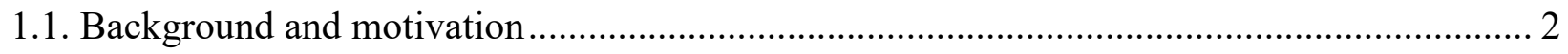

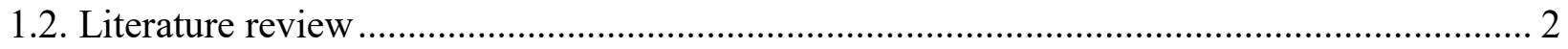

1.2.1. Waterjet technology 3

1.2.2. Solid particle erosion 4

1.2.3. AWJ Cutting of Fiber Polymer Matrix Composites 7

1.2.3.1 Observations when AWJ cutting of Fiber Reinforced Polymers ............................... 7

1.2.3.2 Modelling AWJ cutting of Fiber Reinforced Polymers ............................................ 11

$\begin{array}{ll}\text { 1.2.4. Delamination during AWJM } & 13\end{array}$

1.2.4.1 Delamination Observations when AWJM ……....................................................... 13

1.2.4.2 Modelling Delamination during AWJM .................................................................... 14

$\begin{array}{ll}\text { 1.2.5. Summary } & 17\end{array}$

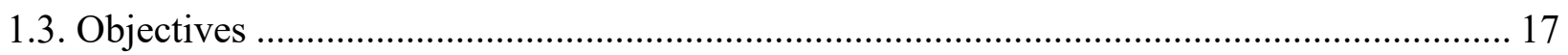

Chapter 2 Prediction of surface roughness in abrasive waterjet trimming of fiber reinforced polymer composites ........................................................................................................................... 19

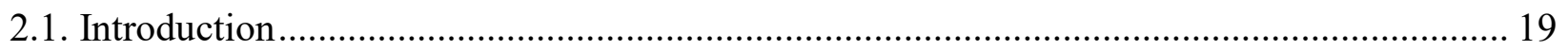

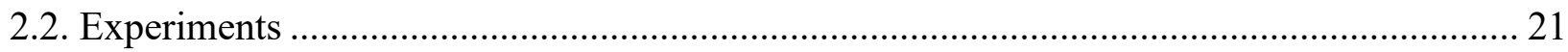

2.2.1. Abrasive Waterjet Machining Experiments 21

2.2.2. Dynamic Hardness Measurement 28

2.2.3. Surface Roughness and Taper Angle Measurements $\quad 30$ 


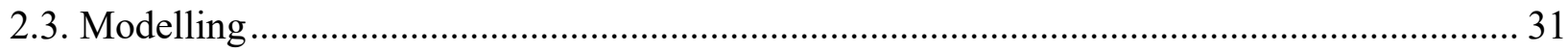

2.3.1. 2D Model Overview 31

2.3.2. Rigid-Plastic Model to Obtain Individual Particle Craters (Block A) 33

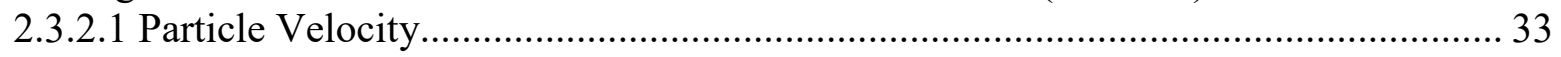

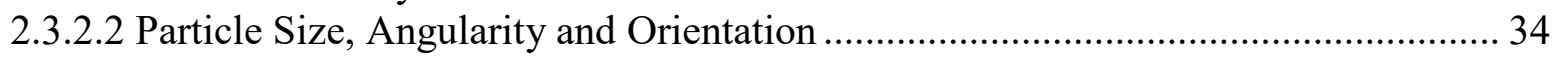

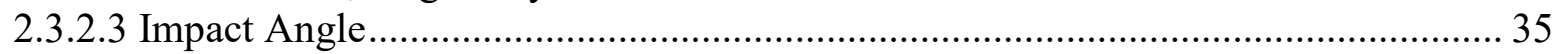

2.3.3. Multiple Particle Impact Profile (Block B) 37

2.3.4. Method to Superimpose Multiple Profiles (Block C) 38

2.3.5.3D Model 41

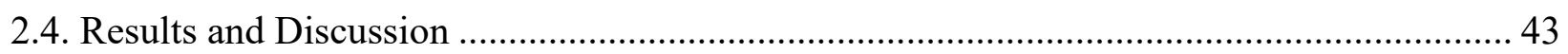

2.4.1. Measured Dynamic Hardness 44

2.4.2. Comparison of Predicted and Measured Roughness for 2D model 44

2.4.3. Effect of AWJ Parameters on Roughness $\quad 50$

2.4.4. Sensitivity Analysis $\quad 56$

2.4.5. 3D Model Results $\quad 57$

2.4.6. Limitations $\quad 59$

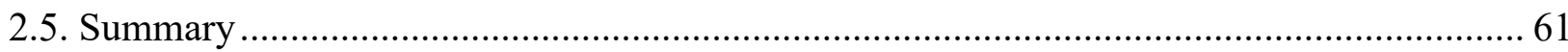

Chapter 3 Modelling of Delamination due to Hydraulic Shock when Piercing Anisotropic

Carbon-Fiber Laminates using an Abrasive Waterjet .................................................................... 62

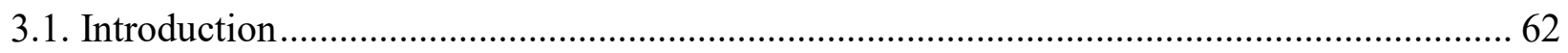

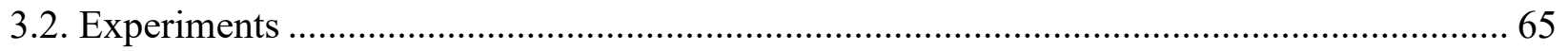

3.2.1. Piercing experiments 66

3.2.2. Quantification of impact delamination 68

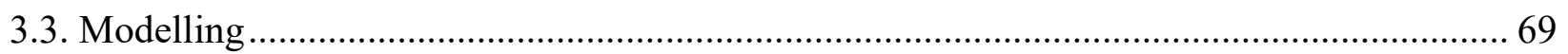

3.3.1. Impact delamination $\quad 70$

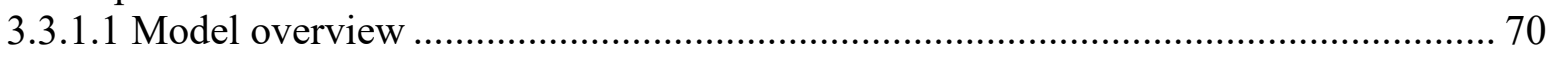

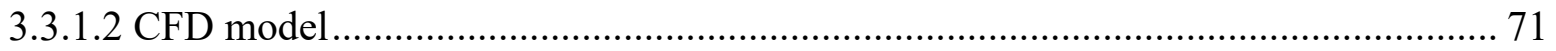

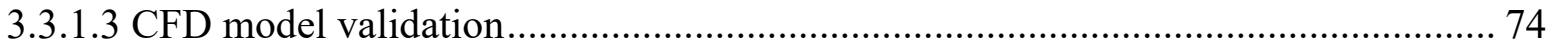

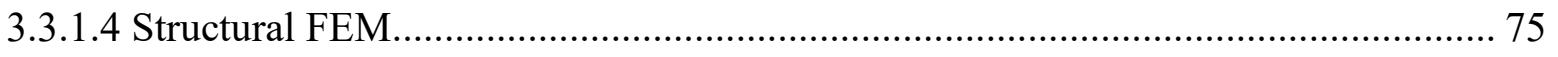

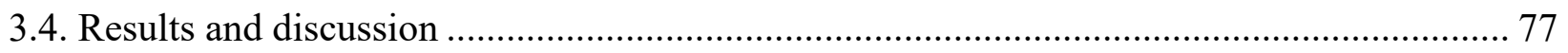

3.4.1. Impact modeling

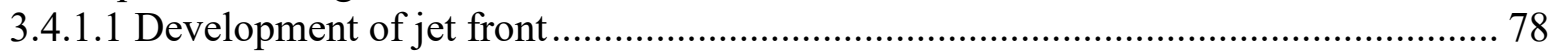

3.4.1.2 Development of hydraulic shock and interaction with substrate ............................. 78

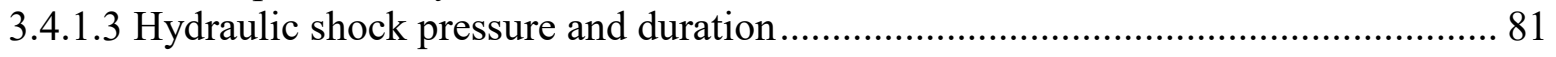

3.4.1.4 AWJ admittance of the CF-epoxy substrate ………................................................... 84

3.4.2. Damage in composite due to AWJ hydraulic shock 86

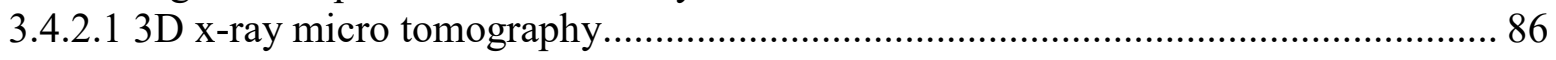

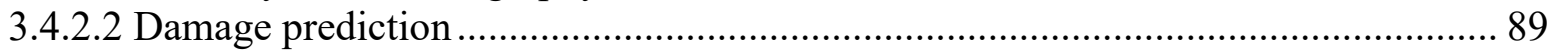




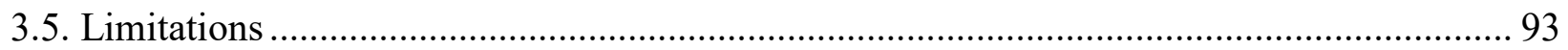

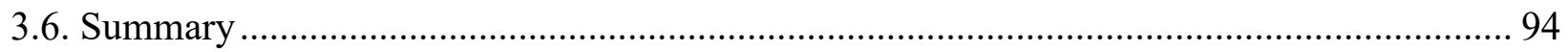

Chapter 4 Characterizing and modelling delamination of carbon-fiber epoxy laminates

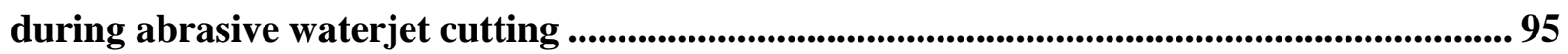

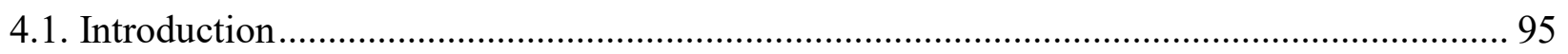

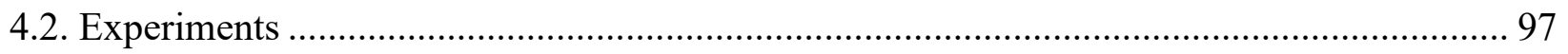

4.2.1. Moisture Uptake Experiment 97

4.2.2. Measurement of Jet Loading Force 101

4.2.3. Micro-tomography and Characterization of the AWJ Cut Geometry 102

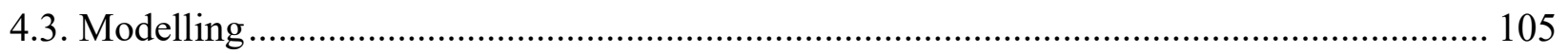

$\begin{array}{ll}\text { 4.3.1. FSI Model Overview } & 105\end{array}$

$\begin{array}{lr}\text { 4.3.2. CFD Model } & 108\end{array}$

$\begin{array}{ll}\text { 4.3.3. Structural Model } & 108\end{array}$

4.3.4. Experimental Results and Discussion 109

4.3.5. Comparison of the Jet Loading for Cutting and Trimming 110

4.3.6. 3D X-ray Micro-tomography of AWJM Through-Cuts 111

4.3.7. Moisture Uptake Results 113

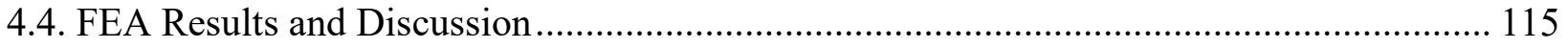

4.4.1. Delamination Onset when AWJ Cutting 115

4.4.2. Comparison of FEA and Moisture Uptake Results 118

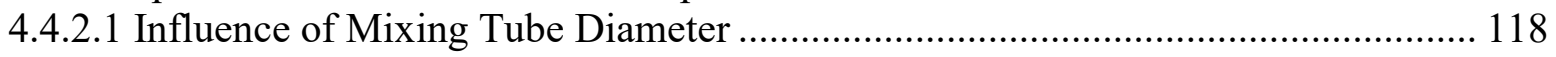

4.4.2.2 Influence of Traverse Speed on Delamination..................................................... 121

4.4.3. Limitations 123

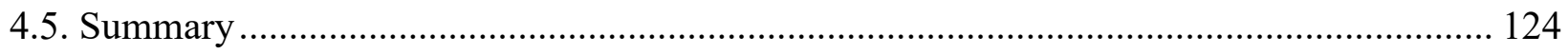

Chapter 5 Summary and Conclusions ................................................................................................... 125

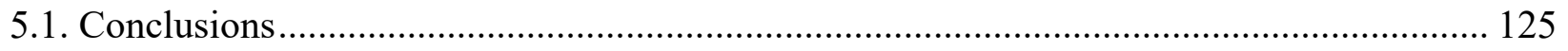

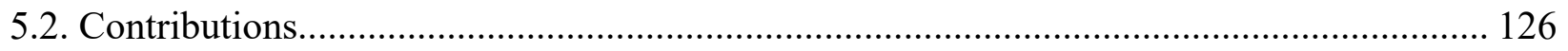

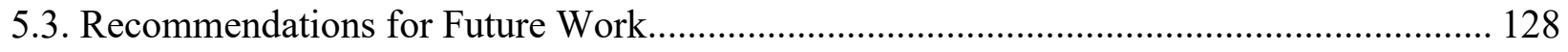

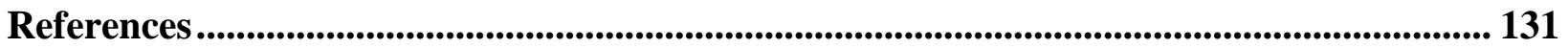




\section{List of tables}

Table 1.1 Effect of various AWJ machining parameters on AWJ cut responses...................... 10

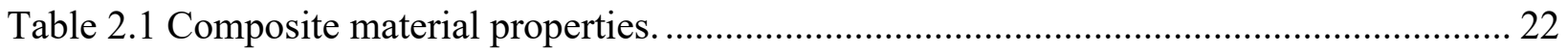

Table 2.2 Experimental conditions, measured taper angles (Fig. 2.3) \pm 1 standard deviation and

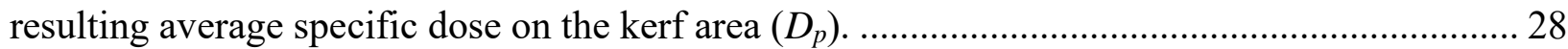

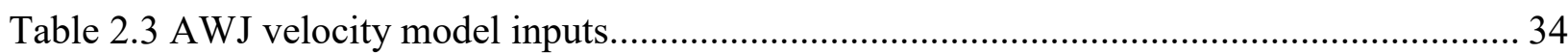

Table 2.4 Dynamic hardness measurements on the target materials. Average particle and crater properties and standard deviations based on 20 measurements......................................... 44

Table 2.5 Model input parameters for sensitivity analysis. ................................................... 56

Table 3.1 Composite material properties as provided by the manufacturer. Moduli and Poission's ratio correspond to properties of a single ply. Tensile strength and tensile modulus corresponded

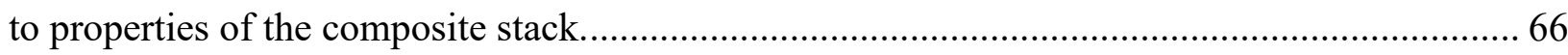

Table 3.2 Experimental conditions for the piercing experiments. ......................................... 68

Table 3.3. Standard $k$ - $\epsilon$ turbulence model [145] and Tait equation parameters [100].............. 73

Table 4.1 Carbon-fiber/epoxy laminate material properties. ................................................ 98

Table 4.2 Factors and levels used in the moisture uptake tests, the x-ray micro-tomography experiments at the two traverse speeds (medium and fast), and the conditions used in the FE models of the kerf for the slow, medium and fast traverse speeds for each nozzle size. Table 4.3 Significant ANOVA results. 'Sig.' represents the probability that data could occur by chance. 


\section{List of figures}

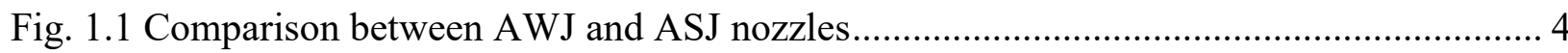

Fig. 1.2 Damage regions associated with AWJ machining of FRP composites (kerf side view) [26].

Fig. 1.3 AWJ machined surface of unidirectional graphite/epoxy composite with different fiber orientations. Fiber Volume fraction $=0.65$, thickness $=5 \mathrm{~mm}$, pressure $=240 \mathrm{MPa}$, Standoff $=1.0$ $\mathrm{mm}$, traverse speed $=1.6 \mathrm{~mm} / \mathrm{s}$, nozzle diameter $=1.0 \mathrm{~mm}[28]$.

Fig. 1.4 LEFM delamination model schematic. $H$ is thickness of the laminate, $a$ is the radial size of the assumed existing crack, and $h$ is the uncut depth [44]. 15

Fig. 1.5 Cuts made by UHP water jets. a) waterjet only, b) $3 \mathrm{~g} / \mathrm{s}$ abrasive jet with no delay of abrasive entrainment, and c) $3 \mathrm{~g} / \mathrm{s}$ abrasive jet with 3 -second entrainment delay. Pressure $=210$ MPa [46]. 16

Fig. 2.1 Abrasive particle size distribution (projected area diameter). Inset micrograph of particle shape. 23

Fig. 2.2 a. Schematic representation of trimming at four different trim widths (note that in the back view of part a, the jet is traversing into the page). $b$. The effect of trimming width on kerf roughness. Material: CF-SMC, pressure: $225 \mathrm{MPa}$, SOD: $1 \mathrm{~mm}, m a=0.2 \mathrm{~g} / \mathrm{s}$, abrasive: 75 micro garnet. Error bars show the variation in surface roughness among three specimens. 25 Fig. 2.3 Schematic of AWJM edge trimming and the location of the profilometer scan used to measure $\mathrm{R}_{\mathrm{a}}$ on the kerf of the trimmed edge.

Fig. 2.4 Size distribution of steel shot used to measure target dynamic hardness. The inset shows micrograph of particles. 29

Fig. 2.5 Typical measured taper profile showing measured angle for specimen ID 1 in Table 2.2. Coordinates defined as in Fig. 2.3. 30

Fig. 2.6 Structure of surface roughness model. 32

Fig. 2.7 Schematic of a model particle as it plows a crater in a ductile substrate according to the rigid-plastic model $[18,19]$. $V_{i}$ - impact velocity, $h$ - idealized rhomboid length, $A_{1}$ and $A_{2}$ particle angularity, $\theta$ - particle orientation, $\alpha$ - impact angle, $D$ - particle length, $d$ - particle width.

Fig. 2.8 Impact of an idealized rhomboid striking the kerf of the trimmed edge. The direction of nozzle traverse is normal to the plane of the figure. 36 
Fig. 2.9 Predicted single-particle impact crater on CF-epoxy substrate for a particle with $A_{l}=56.4^{\circ}, A_{2}=33.6^{\circ}, \rho_{p}=4000 \mathrm{~kg} / \mathrm{m}^{3}, \alpha=0.97^{\circ}, \theta=7.6^{\circ}, V_{i}=650 \mathrm{~m} / \mathrm{s}$. Note the large difference in depth and length scales.

Fig. 2.10 Roughness profile of 17 contiguous single-particle impact craters for the CF-epoxy substrate for the conditions of ID 8 in Table 2.2. 38

Fig. 2.11 Comparison of current method vs. additive superposition relative to iteration number for specimen ID 6 in Table 2.2. 39

Fig. 2.12 Method to superimpose profiles. Solid horizontal lines represent means of the profiles.

Fig. 2.13 3D surface model topology generation process. Block D shows an example of a generated 3D conical crater. Block E shows the superposition of the 3D conical crater on a segment of virtual kerf plane. Block F shows the surface topography of the virtual segment after 50000 iterations. Block $\mathrm{G}$ is the $2 \mathrm{D}$ profile extracted from a randomly generated line along the kerf.

Fig. 2.14 Kerfs of composites trimmed with a $\mu$ AWJ: a. CF-epoxy (ID 1 from Table 2.2) b. CFSMC (ID 16 from Table 2.2). Jet traversed from left to right.

Fig. 2.15 Surface roughness profiles for CF-SMC ID 10 in Table 2.2: a. measured $\left(\mathrm{R}_{\mathrm{a}}=2.41\right.$ $\mu \mathrm{m})$, and $\mathrm{b}$. predicted $\left(\mathrm{R}_{\mathrm{a}}=2.76 \mu \mathrm{m}\right)$.

Fig. 2.16 Fourier transform of: a. experimental, and b. model surface roughness profiles corresponding to ID 10 in Table 2.2. The inset shows magnified component of Fourier transform for smaller wavelength.

Fig. 2.17 Measured and predicted: a. maximum amplitude for all specimens shown in Table 2.2. Error bars correspond to maximum and minimum values of three measurements for each experimental specimen.

Fig. 2.18 a. Skewness, and b. kurtosis of the experimental and model profiles in Table 2.2. Error bars represent the difference between the minimum and maximum values of three measurements for each experimental sample.

Fig. 2.19 Experimental data, 2D model and 3D model roughness results for the experiments of Table 2.2. The error bars for the experimental results and the 3D model results correspond to the maximum and minimum surface roughness measurements on the same specimen (based on three and five measurements per specimen for the experimental and 3D model results, respectively). 49 
Fig. 2.20 Measured taper angle $(\beta)$ as a function of the nozzle traverse speed for the two materials $(P=211.5 \mathrm{MPa}, \mathrm{SOD}=1 \mathrm{~mm}, m p=0.5 \mathrm{~g} / \mathrm{s})$ 50

Fig. 2.21 $R_{a}$ vs. taper angle for a. CF-epoxy and b. CF-SMC, corresponding to the experimental conditions in Table 2.2. The horizontal and vertical error bars represent the maximum and minimum within the specimen measurements for the taper angle, and surface roughness, respectively. The dashed lines are the linear trend lines for the experimental and model data. The $\mathrm{R}^{2}$ is for the experimental data only. 51 Fig. 2.22 Predicted (2D model) and measured $\mathrm{R}_{\mathrm{a}}$ as a function of predicted average particle velocity. Experimental values correspond to ID numbers: 1, 4, 7, 10, 13 and 16 in Table 2.2. Error bars show the standard deviation within each value based on three measurements. 53 Fig. 2.23 Contour plots relating kerf projected dose, particle velocity and taper angle for a. CFepoxy, and b. CF-SMC.

Fig. 2.24 $R_{a}$ as a function of target dynamic hardness (based on the average $\mathrm{R}_{\mathrm{a}}$ for all test cases for CF-epoxy and CF-SMC, respectively). Predicted results are from 2D roughness model. ..... 55 Fig. 2.25 Sensitivity analysis for the surface roughness input factors with input parameters corresponding to Table 2.5 . 56

Fig. 2.26 3D surface topography and 2D extracted profiles for ID No. 1 in Table 2.2 for: a. experimental measurement, and b. 3D model results. 58

Fig. 2.27 Experimental vs. model results for two test cases of the PPLG composite machined using $75 \mu \mathrm{m}$ garnet at $m a=0.5$ with $A_{1}=32^{\circ} \pm 22^{\circ}, A_{2}=58^{\circ} \pm 22^{\circ}$. Test 1: $P=242 \mathrm{MPa}, V_{P}=589$ $\mathrm{m} / \mathrm{s}, U=0.0042 \mathrm{~m} / \mathrm{s}, \beta=2.08$. Test $2: P=195 \mathrm{MPa}, V_{p}=524 \mathrm{~m} / \mathrm{s}, U=0.0033 \mathrm{~m} / \mathrm{s}, \beta=1.83^{\circ} \ldots . .60$ Fig. 2.28 Comparison of oblique scanning electron micrographs of the $\mu \mathrm{AWJ}$ cut surfaces for the three composite substrates. 60

Fig. 3.1 Schematic impact process of AWJ piercing on laminates. 63

Fig. 3.2 Cross-sections of pierced laminate taken through the major and minor axes of the elliptical damage zone. $0^{\circ}$ plies had C-fibers that were parallel to the $x$-axis.

Fig. 3.3 CFD and structural control volumes used in the FSI model. 71

Fig. 3.4 Measured [146] and CFD predicted maximum impact pressure across the jet/substrate contact surface with respect to time. 75

Fig. 3.5 Mixed-mode bi-linear, traction-separation relationship for cohesive elements [149] .... 77 
Fig. 3.6 FSI results of experiment 1 in Table 3.2 for: a. time-steps $0.25-2.12 \mu$ s showing the VF frames during its flight time across the SOD and corresponding to the $z y$-plane of the entire domain space as shown in Fig. 3.3 (circles show magnified views of the jet front), and, b. magnified results (corresponding to the region within the dashed box in a.) from time-steps $t=$ $2.12-2.20 \mu \mathrm{s}$ showing the hydraulic shock action. The VF frames of each time step show the shape and morphology of the waterjet during impact. Similarly, the Pressure/EES frames show the pressure generated inside the waterjet, and the equivalent elastic strain (ESS) response due to the waterjet loading, at each time step. 80

Fig. 3.7 CFD results of the maximum pressure exerted on the substrate for: (a) the $254 \mu \mathrm{m}$ nozzle and (b) the $381 \mu \mathrm{m}$ nozzle. ' $\mathrm{X}$ ' marks the endpoint of the hydraulic shock, which is defined later in the current section.

Fig. 3.8 Maximum hydraulic shock pressures predicted by CFD model and water hammer equations of Cook (equation 3.1)[101], and Heymann (equation 3.14)[108] 82 Fig. 3.9 Jet front axial velocity profiles as a function of mixing tube radius. 84

Fig. 3.10 Variation of impact pressure along the substrate surface when pressure was maximum for a. isotropic polyethylene, b. anisotropic model of the CF-epoxy, and c. isotropic model based on weight-fraction averaged moduli. Isotropic pressure contours generated using the same FE model as specimen 1 in Table 3.2, but with isotropic properties. Anisotropic pressure contour taken from specimen 1 in Table 3.2. Diameter of pressure zone was $104 \mu \mathrm{m}$. 85

Fig. 3.11 Target surface deformation at maximum deflection for the a. isotropic (polyethylene), b. anisotropic (CF-epoxy), and c. weight-fraction averaged isotropic cases, along the centerline of the $x z$ and $y z$-planes, respectively. 86

Fig. 3.12 X-ray micro-tomography scans along the centerline of the pierced holes along the $x z$ plane for: a. specimen 1, and b. specimen 6 in Table 3.2. Dashed lines represent the lamina interfaces. Jet was incident from the top. 87

Fig. 3.13 Elliptical damage zone area in the top ply as a function of nozzle pressure for the two nozzle diameters.

Fig. 3.14 Comparison of the influence of jet power on the measured top-ply elliptical damage zone (left axis) and FEM top-ply debonding area (right axis) (conditions of Table 3.2). Results normalized by maximum values. Trend lines are to guide the eye. 
Fig. 3.15 Predicted equivalent elastic strain (top row in the $y z$-plane normal to the target surface) and debonding (bottom row in the $x y$-plane of the target surface) at key time steps during the AWJ impact simulation zoomed to plies 1 and 2. Debonding is shown as the dark areas in the delamination row. 92

Fig. 3.16 Magnified portion of delamination corresponding to squares in Fig. 3.15 for time steps $\mathrm{t}=2.25 \mu \mathrm{s}$ and $\mathrm{t}=2.29 \mu \mathrm{s}$.

Fig. 3.17 Delamination from a larger $762 \mu \mathrm{m}$ mixing tube nozzle that exceeded the observable space from the micro CT-scans. 93

Fig. 4.1 Schematic of AWJ cutting of the carbon fiber epoxy laminate (not to scale) showing sidewall angle $\beta$. Jet traverse shown parallel to $0^{\circ}$ fibers of the first ply. The CF-epoxy sheet from which the specimens were cut fully supported on the bottom and clamped to the AWJ bed restricting lateral and vertical movement. Final specimen sizes were $85 \times 12.5 \mathrm{~mm} . M_{d}$ is the inner mixing tube diameter. The composite sheet $(30 \times 30 \mathrm{~cm})$ was fixed to the cutting bed along its four edges. 98

Fig. 4.2 Schematic of the force transducer used to measure the loads applied to the cutting front and kerf edges by the water jet. 102

Fig. 4.3 Position and orientation of the orthogonal slices in the CT scans in the vicinity of the cutting front. $X Y$-plane is approximately $200 \mu \mathrm{m}$ from the bottom of the composite sheet....... 103 Fig. 4.4 a. Profilometer scan of the cutting front for medium and fast traverse speeds. The best-fit curves are $3^{\text {rd }}$ degree polynomials. b. Profilometer scan of the kerf taper for medium and fast traverse speeds fitted to straight lines. Height measurements were normalized using the maximum value in each case. Error bars represent maximum and minimum values of 3 measurements at different sections on the same specimen.

Fig. 4.5 Schematics of the three traverse speed case studies and their control volumes for the CFD/FEM coupled analysis: a. slow traverse speed, b. medium traverse speed, c. fast traverse speed. The footprint diameter of $304 \mu \mathrm{m}$ was for the $254 \mu \mathrm{m}$ nozzle. 106 Fig. 4.6 Predicted pressures and CZM inter-ply damage regions on the cut sidewalls from the 1way FSI model for the fast traverse speed. 107 Fig. 4.7 Scanning electron micrograph of delamination in the CF-epoxy substrate as a result of AWJ cutting. Operating conditions: $P=225 \mathrm{MPa}, \mathrm{SOD}=2 \mathrm{~mm}, \mathrm{AFR}=0.93 \mathrm{~g} / \mathrm{s}$, traverse speed: 
$2000 \mathrm{~mm} / \mathrm{min}, 254 \mu \mathrm{m}$ mixing tube diameter and $128 \mu \mathrm{m}$ orifice diameter. Top ply was oriented with fibers perpendicular to the jet traverse; i.e. at $90^{\circ}$ as shown. ......................................... 110

Fig. 4.8 Strain-gauge measurements for the through and trim cut experiments........................ 111 Fig. 4.9 CT scans of AWJ cuts corresponding to the a. fast, and b. medium traverse speeds with operating conditions shown in Table 4.2. Dashed lines encompass delamination damage. Fibers oriented as in Fig. 4.1. The rectangular shaded areas shown in the $x y$-plane indicate the delamination that would be eroded by the advancing cutting front. The depth of the $x y$-planes (right-most micrographs) in the $z$-direction was $\sim 200 \mu \mathrm{m}$.

Fig. 4.10 Scanning electron micrograph of abrasive grains (garnet) wedged into an interlaminar crack along the side-wall of an AWJ cut. Operating parameters were: pressure $=225 \mathrm{MPa}$, $\mathrm{AFR}=0.9 \mathrm{~g} / \mathrm{s}$, and a traverse speed $=4000 \mathrm{~mm} / \mathrm{min}$, using the smallest nozzle size.

Fig. 4.11 Moisture uptake experimental results. 113 Fig. 4.12 Interlaminar stresses for the fast traverse and smallest nozzle. Flags represent the location of maximum stresses. Fibers have the same orientation as in Fig. 4.1. 116

Fig. 4.13 Comparison of predicted $\sigma_{z}$ interface stresses $(\mathrm{Pa})$ with damaged CZM elements for the fast traverse speed case with the $254 \mu \mathrm{m}$ nozzle.

Fig. 4.14 Comparison of experimental damage assessment using moisture uptake and the delamination predicted by the FEA/CZM for three nozzle sizes.. 119 Fig. 4.15 Comparison of predicted $\sigma_{z}$ stress fields $(\mathrm{Pa})$ at the ply interfaces with delaminated CZM elements for nozzle diameters: a. $254 \mu \mathrm{m}$, b. $381 \mu \mathrm{m}$, and c. $762 \mu \mathrm{m}$, at the fastest traverse speed. 120

Fig. 4.16 Comparison of experimental damage assessment using moisture uptake and the delamination predicted by the FEA/CZM for three traverse speeds for the 254 nozzle size. .... 121 Fig. 4.17 Comparison of predicted $\sigma_{z}$ stress field $(\mathrm{Pa})$ at the ply interfaces with disbonded CZM elements for three traverse speeds: a. slow, b. medium, and c. fast, with the $254 \mu \mathrm{m}$ nozzle.... 122 


\section{Nomenclature}

$\alpha$

$\alpha_{L}$

$\beta$

$\beta_{d}$

$\rho$

$\rho_{c}$

$\rho_{L}$

$\rho_{G}$

$\rho_{M}$

$\rho_{s}$

$\rho_{o}$

$\rho_{w}$

$\mu$

$\mu_{G}$

$\mu_{L}$

$\delta_{n}$

$\delta_{n}^{c}$

$\delta_{t}^{c}$

$\delta_{t}$

$\varphi$

$\mu$

$v$

$\lambda$

$\eta$

$\sigma_{Z}$

$\sigma_{k}, \sigma_{\epsilon}$
Angle of impact

Volume fraction of liquid phase

Kerf taper angle

Non-dimensional parameter

Fluid density

Composite density

Density of the liquid phase

Density of the gaseous phase

Target material density

Density of solid

Reference density

Density of water

Viscosity of the mixture

Viscosity of gaseous phase

Viscosity of liquid phase

Normal displacement

Critical normal displacement

Critical tangential displacement

Tangential displacement

Impact angle

Shear modulus of the material

Volume fraction of the material

Dimensional effective displacement parameter

Erosion efficiency

Normal interlaminar stress

Turbulent Prandtl numbers 


\begin{tabular}{|c|c|}
\hline$\sigma_{n}$ & Normal cohesive traction \\
\hline$\sigma_{t}$ & Tangential cohesive traction \\
\hline$\tau_{x z}, \tau_{y z}$ & Shear interalaminar stresses \\
\hline$\theta$ & Particle orientation angle \\
\hline$A$ & Area of delamination \\
\hline$A F R$ & Abrasive flow rate \\
\hline$A_{o}$ & Area of nozzle orifice \\
\hline$a$ & Existing crack length \\
\hline C & Maximum crack length \\
\hline$C_{d}$ & Discharge coefficient \\
\hline$C_{1 \epsilon}, C_{1 \epsilon}, C_{\mu}$ & Turbulence model constants \\
\hline$c$ & Fluid speed-of-sound \\
\hline$c_{2}-c_{6}$ & Constants in brittle erosion model \\
\hline$c_{s}$ & Acoustic velocity of the solid \\
\hline$c_{s, a v e}$ & Average acoustic velocity for a composite solid \\
\hline$c_{w}$ & Acoustic velocity of the water \\
\hline$d$ & Diameter of the orifice \\
\hline$D_{a}$ & Elliptical damage area \\
\hline$D_{m}$ & Damage parameter \\
\hline$d_{j}$ & Vena-contracta diameter of the jet \\
\hline$d_{p}$ & Geometric mean particle diameter \\
\hline$E$ & Modulus of elasticity \\
\hline$E_{c}$ & Average modulus of elasticity \\
\hline$E_{11}$ & Longitudinal Young's modulus \\
\hline$E_{22}$ & Transverse Young's modulus \\
\hline$E_{33}$ & Normal Young's modulus \\
\hline$E_{n}$ & Erosion number \\
\hline$E_{t}$ & Target materials Young's modulus \\
\hline
\end{tabular}




\begin{tabular}{|c|c|}
\hline$F$ & Force the jet exerts at the bottom of a blind hole \\
\hline$F_{x}, F_{y}, F_{z}$ & Force vectors \\
\hline G & Energy release rate \\
\hline$G_{I C}$ & Critical energy release rate \\
\hline$G_{I c}$ & Mode I critical energy release rate \\
\hline$G_{I}$ & Mode I energy release rate \\
\hline$G_{I I c}$ & Mode II critical energy release rate \\
\hline$G_{I I}$ & Mode II energy release rate \\
\hline$G_{n}$ & Normal fracture energy \\
\hline$G_{t}$ & Tangential fracture energy \\
\hline$G_{c n}$ & Normal critical fracture energy \\
\hline$G_{c t}$ & Tangential critical fracture energy \\
\hline$h$ & Uncut depth of the material under the waterjet \\
\hline$H_{M}$ & Target material hardness \\
\hline$H_{v}$ & Material hardness \\
\hline$K_{I C}$ & Target material fracture toughness \\
\hline$K_{o}$ & Reference bulk modulus \\
\hline$K_{n}$ & Normal stiffness \\
\hline$K_{t}$ & Tangential stifness \\
\hline$L_{D}$ & Longitudinal direction \\
\hline$m_{m}$ & Erosion-debris mass flow rate \\
\hline$m_{p}$ & Abrasive particle mass \\
\hline$M_{P}$ & Poisson's ratio of the material \\
\hline$\dot{m}_{s}$ & Slurry mass flow rate \\
\hline$n$ & Density exponent \\
\hline$P$ & Water pressure \\
\hline$p_{d}$ & Dynamic hardness \\
\hline$P_{o}$ & Abrasive waterjet power \\
\hline
\end{tabular}




\begin{tabular}{|c|c|}
\hline$p_{o}$ & Reference pressure \\
\hline$p_{i}$ & Impact hydraulic pressure ('water hammer pressure') \\
\hline$p_{s}$ & Hydraulic pressure from Heymann equation \\
\hline$Q$ & Mass flow rate \\
\hline$R$ & Jet radius \\
\hline$R_{H}$ & Hole radius \\
\hline$R_{p}$ & Nominal particle radius \\
\hline$S, S O D$ & Stand-off Distance \\
\hline$T_{D}$ & Transverse direction \\
\hline$t$ & Time \\
\hline$U$ & Traverse speed \\
\hline$U_{e}$ & Strain energy stored in the material body \\
\hline$v$ & Fluid velocity \\
\hline$V$ & Impact velocity \\
\hline$v_{e l}$ & Collision velocity at which the elastic limit is reached \\
\hline$V_{m}$ & Volume of material removed \\
\hline$V_{\text {Mdef }}$ & Material removal volume during deformation wear \\
\hline$V_{t}$ & Traverse speed \\
\hline$v_{p}$ & Abrasive particle velocity \\
\hline$W_{f}$ & Fiber weight fraction \\
\hline$x$ & Displacement of bottom ply due in the axial direction \\
\hline
\end{tabular}




\section{Chapter 1 Introduction}

This is a manuscript-based dissertation is based on the following publications:

1) J. Schwartzentruber, J. K. Spelt, and M. Papini, "Prediction of surface roughness in abrasive waterjet trimming of fiber reinforced polymer composites," International Journal of Machine Tools and Manufacture, vol. 122, pp. 1-17, 2017. (Schwartzentruber conducted the trimming experiments, analyzed the results, developed the MATLAB component of the 2D and 3D surface roguhness models, and led the writing of the manuscript. The rigidplastic MATHCAD model was developed by Papini).

2) J. Schwartzentruber, J. K. Spelt, and M. Papini, "Modelling of delamination due to hydraulic shock when piercing anisotropic carbon-fiber laminates using an abrasive waterjet," submitted to International Journal of Machine Tools and Manufacture. (Schwartzentruber conducted the piercing experiments, analyzed the x-ray microtomography results, developed the finite element models, and led the writing of the manuscript. The x-ray tomography of the specimens were done by Mr. Shiang Law, from the University of Toronto).

3) J. Schwartzentruber, J. K. Spelt, and M. Papini, "Characterizing and modelling delamination of carbon-fiber epoxy laminates during abrasive waterjet cutting," submitted to Composites Part A: Applied Science and Manufacturing. (Schwartzentruber conducted the cutting experiments, analyzed the x-ray micro-tomography and moisture uptake results, designed and fabricated the pressure transducer, developed the finite element model, and led the writing of the manuscript. The x-ray tomography was conducted by Shiang Law, from the University of Toronto. The moisture uptake results were generated by the MAGNA PPD Lab (Concord, ON, CA), under the guidance of John Ingram and Keith Ward). 


\subsection{Background and motivation}

The applications of composite materials in the automotive, aerospace, structural and biomedical industry have steadily risen due to their superior specific strength and stiffness properties. However, the distinct multiphase structure of composite materials makes them difficult to machine. For example, Mackey found that machining composite materials with conventional cutting tools cause drastic wear on the cutting tools and damages the material in the form of chipping, cracking and delamination [1]. A study using $\mathrm{CO}_{2}$ laser cutting of a polymer matrix showed that the cut quality was extremely poor, producing large dimensional errors, exhibiting excessive burr formation, and resulting in heat affected zones (HAZ) that led to severe thermal damage [2].

Abrasive waterjet machining (AWJM) uses the mechanism of single particle impact erosion to rapidly remove material from a target substrate by accelerating particles using an ultra-high pressure (UHP) water jet. AWJM of composite materials is an attractive method due to its many advantages over traditional methods such as no heat-affected zones (HAZ), no tooling wear, rapid etch rates and the ability to cut virtually any composite material. Although AWJM technology has many benefits over competing technologies when it comes to machining composites, it is prone to producing delamination and variable surface finishes when cutting and piercing these materials. It is thus of great interest to develop principles, models, and practices to reduce damage and increase cut quality when AWJM of composite materials.

\subsection{Literature review}

This section presents a brief literature review of previous studies regarding AWJM composite materials and investigations into its modelling. The objective of this section is to illustrate the areas where further research is required. More extensive literature reviews on specific topics are provided at the beginning of Chapters 2-4. 


\subsubsection{Waterjet technology}

Waterjet technology is an umbrella term that refers to three types of jets: pure waterjets, abrasive waterjet (AWJ) and slurry suspension jet (ASJ). These technologies follow the same basic principles but employ different abrasive and mixing mechanisms.

Pure waterjets achieve high-speed flow by pumping water through an orifice at very high pressures [3]. The first waterjet design was patented by Leslie Tirrell [4] in 1940. Due to the technological limitations of that era, the application of Tirrells' design was limited to surface finishing. Not until the 1970s did pumping methods evolve enough to produce commercially viable 400-MPa pumping systems [3]. However, these waterjets were limited to cutting soft materials due to the relatively small erosion power they generated. The natural evolution of the waterjet system was to entrain abrasives into the waterjet flow. This method would drastically increase the erosion power of the cutting jet. AWJs were commercialized in the mid-1980s, but due to the lack of precision controllers and mechanisms, their use was limited to rough cutting hard-to-machine materials [3].

Both AWJs and ASJs have been developed (Fig. 1.1). These systems are distinguished by whether the abrasive is entrained into the flowing water as a dry powder in an air stream or whether it is entrained as a liquid slurry (e.g. Miller [5], Haghbin et al. [6]). The work presented in this thesis focuses on UHP (ultra-high pressure) $\mathrm{AWJ} /$ micro-abrasive waterjet machining ( $\mu \mathrm{AWJM}$ ) using an abrasive-air injection system. The power of an AWJ is dependent on the flow rate and velocity of the exiting fluid, which are primarily dictated by the size of the orifice and pump pressure. Orifice sizes typically can range from 0.1-1.2 $\mathrm{mm}$ and operate a pump pressures between $150-600 \mathrm{MPa}$. 


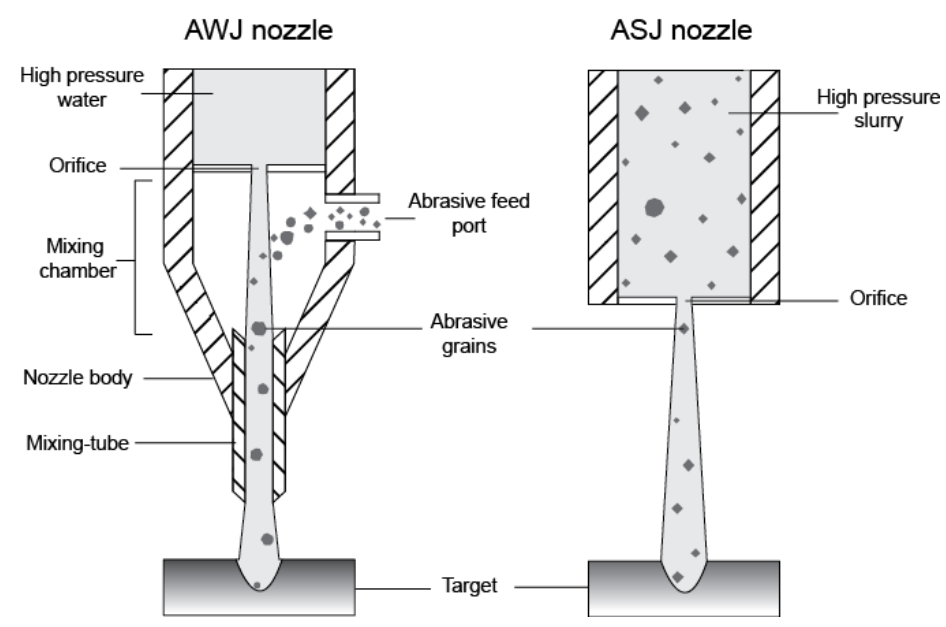

Fig. 1.1 Comparison between AWJ and ASJ nozzles

Since the 1980s, AWJM technology has become more developed, versatile and accepted as a viable manufacturing technology. AWJM applications now include milling, turning, drilling and thin wafer machining [7]. This versatility has made AWJM a useful manufacturing process in many fabrication industries.

\subsubsection{Solid particle erosion}

Solid particle erosion is the fundamental principle governing material removal in AWJ technology. Finnie [8] and Bitter [9, 10] identified many of the fundamental material removal mechanisms. Solid particle erosion is a wear mechanism where the material is deformed/removed due to repetitive contact between impinging particles and a target. As the number of contacts (the dose) rises, so does the amount of erosion. AWJM systems exploit this by accelerating the erosion phenomena to rapidly remove material.

Erosion is affected by many different parameters. One important parameter is the dependence of erosion on the particle impingement angle. It has been determined that brittle materials show peak erosion at high impact angles (normal to the target surface), while ductile materials erode more at shallower impact angles (oblique angles $\sim 30^{\circ}$ ) [9, 11-14]. Erosion of brittle materials is most predominant at impact angles perpendicular to the target and is commonly known as deformation wear. The kinetic energy of the particles upon impact is 
converted into elastic deformation energy which generates fractures. The material removal mechanism in a brittle material is thus due to crack propagation and chipping.

Upon impact of a spherical particle on a target material (either brittle or ductile), the highest stress concentration occurs at the center of the contact area. If the stress maximum does not reach the yield strength of the material, only elastic deformation occurs and the impact is perfectly elastic. Repetitive elastic impacts should cause no damage besides fatigue damage. For ductile materials, if the stress concentration exceeds the material yield strength, then a plastic region forms and hardens the material, thus increasing the elastic limit of the material due to strain hardening. If plastic deformation continues, the limit will eventually become as large as the material strength. Once the material strength is reached, the impact surface will become relatively hard and brittle and unable to deform plastically. At this point, the surface layer is destroyed and fragments are removed. In comparison to brittle materials where plastic yielding is minimal yet present, the erosion occurs from chipping due to crack propagation and usually begins from defects in the microstructure.

Momber and Kovacevic [7] state that solid particle erosion is generally characterized by the erosion number, $E_{n}$, which is equivalent to the quotient of erosion-debris mass flow rate $\left(m_{m}\right)$ to the abrasive particle mass $\left(m_{p}\right)$ as shown the equation (1.1).

$$
E_{n}=\frac{m_{m}}{m_{P}}
$$

Thus the volume of material removal $\left(V_{m}\right)$ per solid particle impact can be expressed as

$$
V_{M}=\frac{E_{n} \cdot m_{P}}{\rho_{M}}
$$

where $\rho_{M}$ is the target material density [7]. Bitter [9] solved for this material removal volume during deformation wear $\left(V_{M d e f}\right)$ as:

$$
V_{M d e f}=\frac{m_{P} \cdot\left(v_{P} \cdot \sin \varphi-v_{e l}\right)^{2}}{2 \cdot \varepsilon_{M d e f}}
$$


where $v_{p}$ is the abrasive particle velocity, $\varphi$ is the impact angle and $v_{e l}$ is the velocity of collision at which the elastic limit is reached. The term $\varepsilon_{M d e f}$ is also known as the deformation wear factor, i.e., the amount of energy needed to remove a unit volume of material from the body surface by deformation wear. Equation (1.3) is only valid if deformation wear occurs [9]. It is worth mentioning that not all energy during plastic deformation is absorbed into lattice distortion. Heat generation occurs during the impact process and liberates some of the energy [10].

Finnie [8] developed a model to predict material removal of a single rigid particle impacting a ductile (plastic) target, which was later extended by Hutchings [15], resolving the kinematics for spherical and square particles under normal and oblique impact. The accuracy of the work regarding spherical particle impact was subsequently improved by Hutchings et al. [16] and Rickerby and Macmillian [17] when predicting energy loss, crater volume and collision kinematics. The square particle impact model of Hutchings [15] was later generalized by Papini and Spelt $[18,19]$ to account for rhomboids of variable angularity and target material dynamic hardness. It was shown that the model was capable of predicting the rebound trajectories of idealized rhomboid particles as they ploughed through an aluminum target [20].

With respect to single particle impact erosion on composite materials, Patnaik et al. [21] developed a theoretical, multi-particle impact model to predict the erosion rate on a glasspolyester cross-ply composite. The theoretical erosion wear rate $\left(E_{r t h}\right)$ was defined as,

$$
E_{r t h}=\frac{\rho_{c} \eta V^{2} \sin ^{2} \alpha}{2 H_{v}}
$$

where $\rho_{c}$ is the density of the composite, $\eta$ is the experimentally determined erosion efficiency, $V$ is the impact velocity, and $H_{v}$ is the hardness of the material. 
Brittle erosion models are generally well developed because of extensive research performed on indentation fracture mechanics. Most empirical brittle erosion models are in the following general form [7]

$$
V_{M}=C_{1} \cdot\left(\frac{d_{P}}{2}\right)^{C_{2}} \cdot v_{P}^{C_{3}} \cdot H_{M}^{C_{4}} \cdot K_{I c}^{C_{5}} \cdot E_{t}^{C_{6}}
$$

where $d_{p}$ is the geometric mean particle diameter, $H_{M}$ is the target material hardness, $K_{I c}$ is the fracture toughness, $E_{t}$ is the material's Young's modulus, and $C_{1}-C_{6}$ are constants solved via experimental fitting. The exponents $c_{2}-c_{6}$ vary depending on the model being used. The validity of equation (1.5) has be confirmed through the research of Wiederhorn and Lawn [22] who developed a quasi-static lateral crack model for sharp particles impacting glass, Evans et al. [23] who developed a numerical model for impact damage in brittle materials, Marshall et al. [24] when modelling lateral cracks during sharp indentation of ceramics, and Buijs [25] when developing an indentation model for glass.

\subsubsection{AWJ Cutting of Fiber Polymer Matrix Composites}

\subsubsection{Observations when AWJ cutting of Fiber Reinforced Polymers}

AWJM produces three distinct regions on cut surfaces of fiber reinforced polymer (FRP) composites: an initial damage region (IDR), a smooth cutting region (SCR) and a rough cutting region (RCR) [26]. These regions are shown schematically in Fig. 1.2. The IDR is characterized by the rounded jet entrance commonly associated with AWJM due to the secondary slurry erosion of the escaping slurry mixture. The SCR is the region where striations are yet to appear since the abrasive particles maintain a high energy. The RCR begins when striations occur, and increases in waviness and roughness become more apparent as the jet increases in depth. Ramulu and Arola [27] have also observed these cutting regions, but have classified them as the initial impact zone, cutting wear zone and deformation wear zone. 


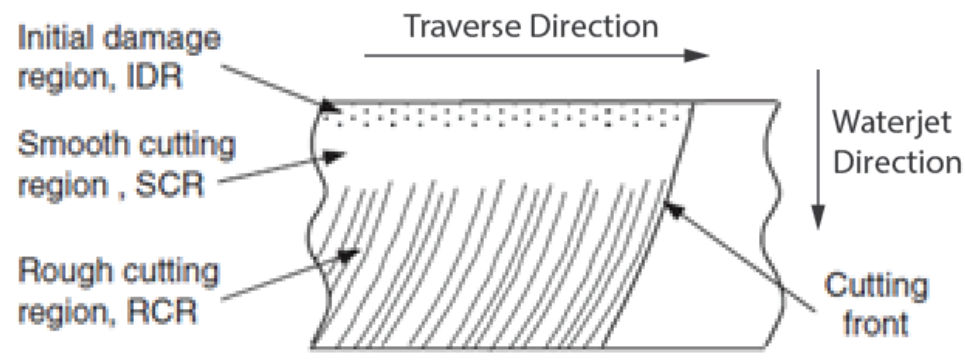

Fig. 1.2 Damage regions associated with AWJ machining of FRP composites (kerf side view) [26].

Scanning electron micrographs (SEM) of an AWJ a graphite/epoxy composite (IM6/3501-6) cut using an AWJ for fiber orientations of $0^{\circ}, 45^{\circ}$, and $90^{\circ}$ are shown in Fig. 1.3 [26]. The results suggest that the primary mode of material removal of the fibers was fracturing by either shear fracture or micro-bending near the jet exit; while micro-cutting of the fibers and matrix were predominant in the SCR. The micrographs revealed the absence of matrix between the cut fiber ends. This result indicates that the weaker matrix material was eroded more readily by the waterjet. The cutting of the $45^{\circ}$ unidirectional composite produced more pronounced striations than that of the $0^{\circ}$ and $90^{\circ}$ composites [28]. The larger degree of striations was commonly accompanied by a high degree of delamination. The $45^{\circ}$ and $90^{\circ}$ kerf surfaces contained larger cavities due to fiber pull out, whereas the $0^{\circ}$ composite did not exhibit striations, but contained voids due to fiber roll out. The divergence between angled composites and $0^{\circ}$ composites with respect to striations is expected under identical machining parameters since a majority of the erosion causing separation is the matrix material for the $0^{\circ}$ orientation. Since polymer matrix materials are much weaker than their fiber counterpart, they erode more readily causing cleaner separation. 


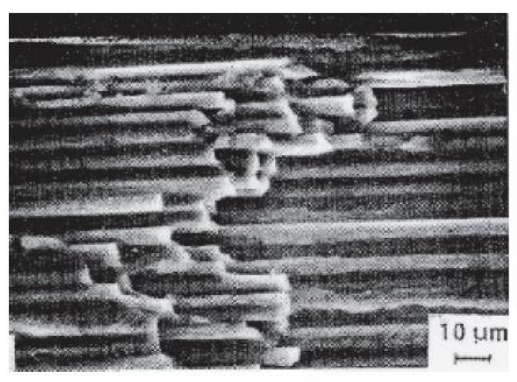

(a) $0^{\circ}$

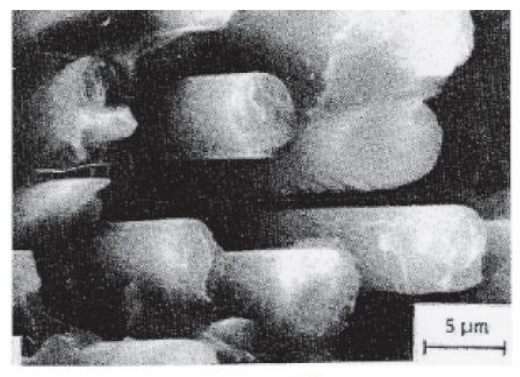

(b) $45^{\circ}$

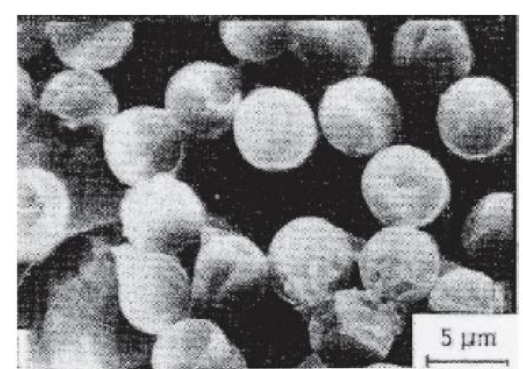

(c) $90^{\circ}$

Fig. 1.3 AWJ machined surface of unidirectional graphite/epoxy composite with different fiber orientations. Fiber Volume fraction $=0.65$, thickness $=5 \mathrm{~mm}$, pressure $=240 \mathrm{MPa}$, Standoff $=1.0$ $\mathrm{mm}$, traverse speed $=1.6 \mathrm{~mm} / \mathrm{s}$, nozzle diameter $=1.0 \mathrm{~mm}[28]$.

It has been observed that a high degree of pull-out irregularities occur in the IDR. SheikhAhmad [29] attributed these irregularities to the abrasive particles at the periphery of the jet, and stated that since particulate density is low at the jet periphery, and the particles have a high energy, thus contributing to the non-uniform damage. This explanation is inconsistent with the work of Ramulu and Arola [27], who observed that abrasive grit size and stand-off distance (SOD) have the most influence on the surface roughness at low cutting depth, and that grit size and traverse speed were most influential in the deformation wear zone, while pressure and abrasive flow rate (AFR) had the least influence of surface roughness at any depth. Liu has shown that the rounded target edges at the jet entrances were due to secondary slurry erosion [30].

A number of investigators have discussed the makeup of the abrasive waterjet itself, which can be helpful in explaining some of these effects. For example, Simpson [31] has shown that the distribution of abrasive concentrations within the jet is saddle-like, i.e., higher at the outer zone of the jet. Simpson [31] also determined that increasing SOD increases the concentration of the particulates towards the jet periphery, while increasing pressure and AFR results in more abrasives being present in the central jet region [31]. Himmelreich and Riess [32] have determined the water-only jet and AWJ velocity profiles along the radial axis of the jet. Their results showed that the water-only jet produces an axial velocity jet plume similar to the Hagen-Poiseuille flow profile, while the abrasive's axial velocity was relatively constant along the diameter of the jet, and not increasing as Sheikh-Ahmad [29] would suggest. Srinivasu and 
Axinte [33] have also shown that the Gaussian velocity profile the water-only jet plume contributes to reduced erosion rates at the edges of the jet. The decrease in water axial velocity near jet edges has also been shown by the CFD analyses of Liu et al. [34].

Based on this previous research, a more reasonable explanation than that proposed by Sheikh-Ahmad for the increase in fiber pullout near the IDR could be attributed to flow directionality. The secondary slurry erosion caused by the jet results in curved particle trajectory which allows the abrasives to attack the edge from a direction other than perpendicular. These changes in particle trajectory are most common in the IDR and the bottom of the RCR. The particle trajectory during the SCR is nearly vertical (i.e., incoming and outcoming jets are parallel to each other), resulting in a smoother surface finish.

Sheikh-Ahmad [26] reported the effect that various machining parameters have on cut quality. The responses are summarized in Table 1.1 .

Table 1.1 Effect of various AWJ machining parameters on AWJ cut responses.

\begin{tabular}{|c|c|c|c|c|c|c|}
\hline \multirow[b]{2}{*}{ Parameter } & \multirow[b]{2}{*}{ Value } & \multicolumn{5}{|c|}{ Response Trend } \\
\hline & & $\begin{array}{l}\text { Entrance } \\
\text { Surface } \\
\text { Roughness }\end{array}$ & $\begin{array}{l}\text { Exit Surface } \\
\text { Roughness }\end{array}$ & $\begin{array}{l}\text { Kerf } \\
\text { Entrance } \\
\text { width }\end{array}$ & Kerf Exit with & Kerf Taper \\
\hline \multirow[t]{2}{*}{$S O D$} & Higher & - & - & Increased & Insignificant & - \\
\hline & Lower & - & - & Decreased & Insignificant & - \\
\hline \multirow[t]{2}{*}{ Pressure } & Higher & - & - & - & - & Decreased \\
\hline & Lower & - & - & - & - & Increased \\
\hline \multirow[t]{2}{*}{ Traverse Speed } & Higher & Increased & Increased & Increased & Increased & Increased \\
\hline & Lower & Decreased & Decreased & Decreased & Decreased & Decreased \\
\hline \multirow[t]{2}{*}{ Grit Size } & Higher & Decreased & Decreased & - & - & Increased \\
\hline & Lower & Increased & Increased & - & - & Decreased \\
\hline
\end{tabular}

Substantial research in AWJ cutting of FRP has focused on qualitatively and quantitatively examining the kerf morphologies with respect to various machining parameters. However, the AWJ nozzle diameters used in previously published research have all been greater than $762 \mu \mathrm{m}$ when machining composite materials. Minimal work has assessed the trends of $\mu \mathrm{AWJM}$ composite substrates, which utilizes nozzles capable of producing micro-features. Due 
to this research gap, studies on $\mu \mathrm{AWJM}$ (i.e., nozzles capable of producing feature sizes less than $500 \mu \mathrm{m}$ ) of composite substrates will be examined in this dissertation.

\subsubsection{Modelling AWJ cutting of Fiber Reinforced Polymers}

Previous work on predicting surface roughness and taper when performing AWJM has mostly been semi-empirical at best. Azmir et al. [35] performed an analysis of variance (ANOVA) statistical analysis on the AWJM of an aramid (Kevlar 129) reinforced phenolic composite, focusing on SOD, AFR, traverse speed and pressure as the factors and the cut surface roughness and taper as the responses. They concluded that the most significant factors contributing to surface roughness and taper were traverse speed and pressure, with a trend indicating that decreasing traverse speed and increasing pressure would both independently decrease surface roughness and taper [35]. In addition to the parametric analysis, they applied a linear regression to predict the roughness and taper values [35]. The empirical models were capable of predicting the surface roughness and taper with a coefficient of determination of 0.828 and 0.736 , respectively.

Besides the fact that their model does not explain the underlying physics of the problem, another critique of their experiment would be with regard to their surface roughness measurement methodology. Azmir et al. [35] took surface roughness measurements at the midline of the cut surfaces, however their material was a laminate consisting of $0^{\circ}$ and $90^{\circ}$ orientations. The surface roughness will likely vary depending on the ply being examined, where $0^{0}$ fiber oriented plies will most likely have a varying surface roughness topology contributed to fiber roll out, and $90^{\circ}$ have a roughness profile due to fiber pull out. Obtaining a roughness profile of the same ply among samples will yield similar results, however the precision of taking the measurement would require very stringent alignment during the profile measurements and near perfect reproducibility during the manufacturing of the composite samples.

Azmir and Ahsan [36] published another very similar statistical analysis to the cutting of the Kevlar composite but based it on the cutting of glass/epoxy composites. The glass/epoxy samples were chopped fiber and woven ply laminates. In addition to the previous factors

(pressure, SOD, AFR, and traverse rate), Azmir and Ahsan also introduced the factors of cutting 
direction (the various cutting orientations were $0^{\circ}, 22.5^{\circ}$, and $45^{\circ}$ ) and abrasive type (garnet and aluminum oxide), and analyzed their effects on surface roughness. They concluded that the most significant control factor on surface roughness during AWJ machining was the type of abrasive used, where the harder alumina abrasive fractured more than the garnet on contact, thus reducing the surface roughness [36]. The optimal cutting conditions for reducing surface roughness were the ones that produced the highest jet kinetic energy on impact (i.e. highest hydraulic pressure, smallest SOD, lowest traverse rate and largest AFR) [36]. Their analysis of cutting orientation proved that it was an insignificant factor. The insignificance of the cutting orientation makes sense in this investigation since the fiber orientations were random and woven, with no distinct direction, unlike unidirectional composites. In 2009, Azmir and Ahsan [37] expanded on the abrasive water jet cutting of glass/epoxy laminates focusing on surface roughness and kerf taper. They delivered very similar results to their previous experiment.

Wang [2] performed a comprehensive analysis of AWJ cutting of polymer matrix composites. His design of experiments consisted of a 4 level 3 factor set, with variation of the pressure, traverse speed, and abrasive flow rate. He also examined the effects of jet inclination angle with respect to various pressures and traverse speeds. Due to external influences, the jet angle experiments were not consistent with respect to the 4 level 3 factor experiments in terms of abrasive flow rate, and this added discrepancies between the results. Wang's [2] investigation also assessed the effect jet angle had on the depth of cut, the smooth cutting region depth, the kerf width, the kerf taper angle, and the surface roughness. His results showed that the depth of cut increased when the trajectory of the jet became more perpendicular. In Wang's [38] subsequent analysis, the maximum depth of cut was observed at an $80^{\circ}$ jet angle. The smooth cutting region was relatively unaffected by the jet angle. The kerf width only varied by approximately $100 \mu \mathrm{m}$ between the $50^{\circ}$ and $90^{\circ}$ orientations, leading to the conclusion that the jet angle was independent of kerf width. Surface roughness showed a considerable improvement (approximately $50 \%$ reduction) when the jet angle was increased from $50^{\circ}$ to $70^{\circ}$ [2]. Based on the observations, Wang developed predictive empirical models for top kerf width, kerf taper angle, surface roughness and depth of smooth cutting region using a statistical regression. The results of the regression models had all coefficients of determination greater than 0.8 . 
Wang's investigation in the cutting of polymer composites was extensive but did not assess the material removal rate (MRR) associated with the erosion of the target. It is known that ductile materials erode more efficiently at angles of impingement of $\sim 30^{\circ}$, and thus examining the depth of cut only does not completely justify the effectiveness of a jet. Shortly after, Wang and Guo [38] developed a semi-empirical model for depth of cut when AWJM composite substrates using a jet energy approach. Their results were in good agreement with experimental values and capable of predicting the depth of cut at varying operating conditions.

Shukla and Tambe [39] modelled the surface roughness and kerf widths when AWJ cutting Kevlar composites using neural networks. However, the inputs of the neural network were based on experimental results, from which the model would perform an optimized statistical analysis. The results of the model were in good approximation of the experimental values.

In summary, the literature regarding the modelling of various surface kerf morphologies during AWJ cutting of composite materials has been heavily regression based. Surface roughness models are mostly based on experimental trials. Due to the variability to AWJM apparatuses and environmental conditions, the applicability of these semi-empirical models is limited. The work in this dissertation will address the need for a theory-based surface roughness model during AWJM, which has not been accomplished before.

\subsubsection{Delamination during AWJM}

\subsubsection{Delamination Observations when AWJM}

Delamination of plies within laminates commonly occurs when cutting composite materials. Sheikh-Ahmad [29] states that low pressure and fast traverse speeds increase the amount of delamination present in the composite structure [26]. It is also noted that in these cases, large striations are seen in the material at the jet exit due to losses in erosive potential. Colligan et al. [40] determined that there exists a critical traverse rate and abrasive flow rate for a given set of machining parameters that promotes ply separation. Koenig et al. [41] had comparable results 
when machining $3 \mathrm{~mm}$ aramid-glass, fiber reinforced polymer (FRP) at a jet pressure of 350 $\mathrm{MPa}$ and a SOD of $2 \mathrm{~mm}$.

Hashish [42] performed multiple experiments on cutting, turning, milling and drilling composite materials. He determined that by reducing the jet diameter and supporting the material at the bottom, delamination could be reduced.

Observations of delamination during AWJM have thus far been limited to qualitative analysis using scanning electron microscopes. The research in this dissertation will fill this gap, by developing a methodology that will quantitatively assess delamination after AWJM using moisture uptake testing and the use of x-ray microtomography.

\subsubsection{Modelling Delamination during AWJM}

Ho-Cheng [43] described the delamination during drilling by waterjet piercing using a fracture mechanics approach with plate theory. He proposed a model that related delamination damage to waterjet pressure and laminate properties, and observed that delamination occurs near the exit of a through-pierce as a result of the reducing strength of the uncut thickness, as drilling depth increases [43]. As a result of the reduced strength, bending of the plies occurs before the laminate is completely through-pierced, causing delamination. As an interlaminar crack is made, water rushes into the opening creating a hydrodynamic pressurization. Koenig et al. [41] have attributed this as a possible cause for damage around the holes when water jet drilling. In summary, Ho-Cheng [44] described the delamination as two events: the first event is the interlaminar separation caused by the thrust of the jet, and the second event is the propagation of delamination damage by hydrodynamic pressurization. These relations only apply to laminated composites. Shanmugam et al. [45] also supports the two event delamination process.

Ho-Cheng's [44] model uses linear elastic fracture mechanics (LEFM), since composite materials show high moduli of elasticity and a lower degree of plasticity. The model utilizes the Energy Release Rate $(G)$ in order to quantify the delamination as shown in equation (1.6) [43].

$$
G d A=F d x-d U_{e}
$$


The right hand side term, $F d x-d U_{e}$, is the rate of change of the potential energy in an elastic body, commonly notated as $\Pi$, where $F$ is the force the jet exerts, $x$ is the displacement, $U_{e}$ is the stored strain energy in the material body and $d A$ is the change in the area of delamination. The incremental area of delamination $d A$ can be further expressed as

$$
d A=\pi(D+2 a) d a
$$

These terms are graphically represented in Fig. 1.4.

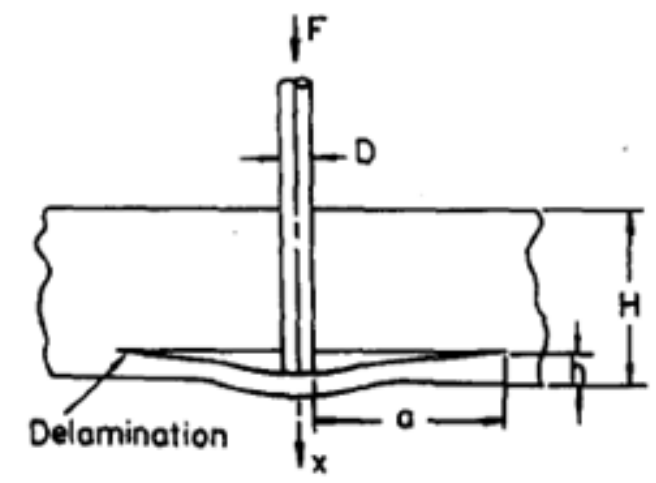

Fig. 1.4 LEFM delamination model schematic. $H$ is thickness of the laminate, $a$ is the radial size of the assumed existing crack, and $h$ is the uncut depth [44].

Ho-Cheng [44] correlated the displacement, force and strain energy using Kirchhoff's classic plate theory for a circular plate with a concentrated load and clamped ends. The critical waterjet pressure at the onset of delamination for an existing interlaminar crack was given by

$$
P^{*}=\sqrt{\frac{8 E G_{I C}}{3 R^{4}\left(1-\mu^{2}\right)}} \cdot h^{\frac{2}{3}}
$$

where $G_{I C}$ is the critical energy release rate, $E$ is the modulus of elasticity, $h$ is the uncut depth under the waterjet, $R$ is half the jet diameter, and $\mu$ is the laminate's Poission's ratio. The model was capable of predicting the maximum delamination pressure within $\sim 17 \%$ error, but makes several assumptions that the author admits to which ultimately diminishes its accuracy. HoCheng [44] assumed the material was isotropic to simplify the relationship; however, it is well known that composite materials can be highly anisotropic. 
Shanmugam et al. [45] performed delamination experiments on a graphite epoxy composites focusing on the mechanism that causes delamination, and developed a semiempirical predictive model for delamination. The results from the experiments can be seen in Fig. 1.5.
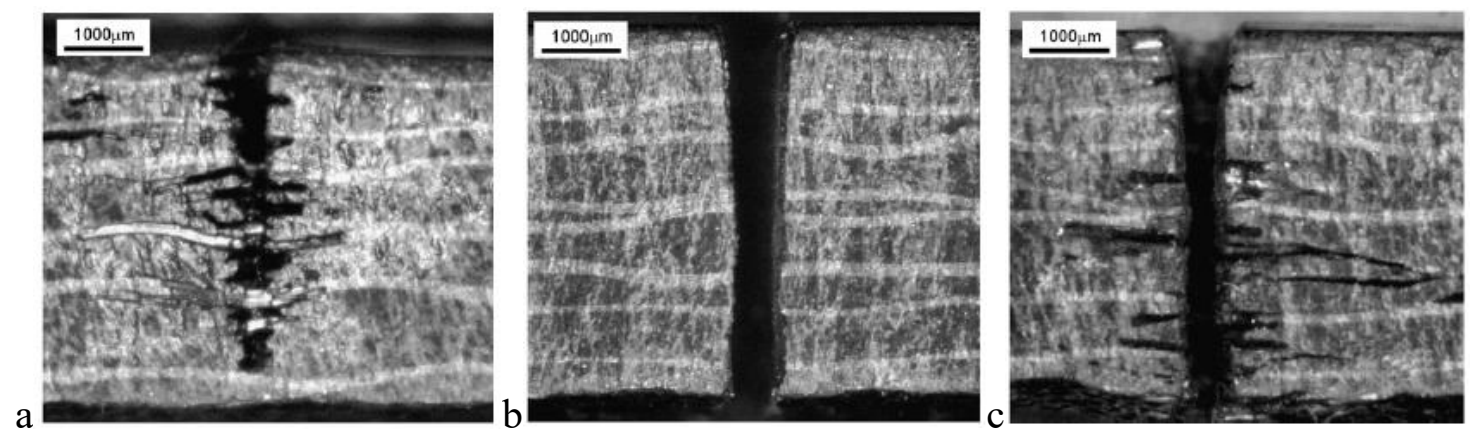

Fig. 1.5 Cuts made by UHP water jets. a) waterjet only, b) $3 \mathrm{~g} / \mathrm{s}$ abrasive jet with no delay of abrasive entrainment, and c) $3 \mathrm{~g} / \mathrm{s}$ abrasive jet with 3 -second entrainment delay. Pressure $=210$ $\mathrm{MPa}$ [45].

The results show that the waterjet only, and delayed entrainment jet produced delaminated cuts. The waterjet only was unable to pierce through the material, while the delayed abrasive entrainment jet wedged abrasive grains between delaminated plies. Shanmugam et al. [45] attribute the poor performance of the waterjet only to its material removal mechanism by shock wave impact. They modeled this failure by impact as a mode I fracture. The fractures developed are then propagated by the hydrodynamic pressurization of the inbound waterjet. The delamination model proposed by Shanmugam et al. [45] is a function of the AWJ operating parameters and material properties, and can be expressed in its general form as

$$
C=f\left(V_{t}, d_{j}, P, S, \dot{m}_{S}, E_{11}, E_{22}, v_{1}, v_{2}, \mu_{1}, \mu_{2}, M_{P 1}, M_{P 2}\right)
$$

where $C$ is the maximum crack length, $V_{t}$ is the traverse speed, $P$ is the water pressure, $S$ is the standoff distance, $\dot{m}_{S}$ is the slurry mass flow rate, $E_{11}$ and $E_{22}$ is the longitudinal and transverse Young's modulus, $v$ is the volume fraction of the fiber and matrix, $\mu$ is the shear modulus, and 
$M_{P}$ are the Poisson's ratio, of the material, where the subscripts 1 and 2 refer to the laminate and laminate bonding, respectively. Similar to the model generated by Ho-Cheng [43], Shanmugam et al. [45] base their model on LEFM, and the energy release rate. Unlike Ho-Chengs' model [44], the model proposed by Shanmugam at al. [45] takes into account anisotropy. The research in the present dissertation focuses more on determining the location of delamination along the depth of a laminate composite during AWJ cutting.

\subsubsection{Summary}

While a significant amount of experimental studies of AWJM of composites have been performed, there are relatively few predictive models of surface roughness and delamination. Most models are empirical or semi-empirical, relying on non-fundamental operating parameters, e.g. pump pressure, making them suitable for only a limited set of process conditions, and specific to the AWJ apparatus and target material being used. The research in this dissertation will focus on predictive modelling of surface roughness and delamination occurring during AWJM cutting and piercing operations, based on knowledge of fundamental operating parameters such as particle velocity, particle shape, stagnation pressure, material properties, etc.

\subsection{Objectives}

The overall objective of this thesis is to develop and verify models to predict the surface roughness and delamination that occur when performing AWJM, as a function of the process parameters. To do so, the following secondary objectives will be accomplished:

Qualitatively and quantitatively assess the kerf surface roughness quality when cutting the composite substrates as a function of $\mathrm{AWJM} / \mu \mathrm{AWJM}$ process parameters. This will assist in understanding the cut surface topology, and define the range of operating parameters throughout testing (Chapter 2).

Develop a surface roughness model based on the AWJ operating conditions using the rigid-plastic model of Papini and Spelt [46] (Chapter 2). 
$>$ Determine the differences in material response between piercing and cutting laminate composites. Piercing with abrasive waterjet causes a water hammer effect that can generated increased delamination. This will serve to separate the two delamination mechanisms (Chapter 3 and 4).

$>\quad$ Conduct moisture uptake tests to determine the extent of delamination with respect to the abrasive waterjet (AWJ) process parameters. The results of this analysis will quantify the extent of delamination during AWJ cutting, and assist in validating the delamination model (Chapter 3)

$>\quad$ Conduct CFD/FEM of the fluid flow at various operating parameters in order to extract force and pressure information needed as inputs for predictive models of cut topography. This will be compared with experimental jet force results to determine the location of crack initiation when AWJ cutting (Chapter 3).

$>\quad$ Develop models to predict CFRP laminate delamination as a function of the AWJ process parameters (Chapter 3 and 4) 


\section{Chapter 2 Prediction of surface roughness in abrasive waterjet trimming of fiber reinforced polymer composites}

This chapter is based on the following published paper:

J. Schwartzentruber, J. K. Spelt, and M. Papini, "Prediction of surface roughness in abrasive waterjet trimming of fiber reinforced polymer composites," International Journal of Machine Tools and Manufacture, vol. 122, pp. 1-17, 2017

\subsection{Introduction}

AWJM uses an ultra-high pressure water jet to entrain a mixture of abrasive particles and air. AWJM is ideal for processing composite materials containing hard reinforcing particles or fibers because it produces no heat affected zone, minimal tool wear, and results in negligible residual stresses and workpiece reaction forces [3]. However, the erosion mechanisms associated with AWJ material removal result in variable surface finish and roughness, depending on the operating conditions [47-50]. Surface roughness resulting from AWJM and $\mu$ AWJM is one of the major quantifying attributes of part quality [51-53]. For example, Liu et al. [54] showed that surface roughness is a major factor in the fatigue life of aircraft aluminum alloy dog-bone specimens produced using AWJM. Annoni et al. [55] showed that surface roughness is very important to the fatigue performance of piezoelectric ceramics processed using AWJM. Therefore, the modelling of surface roughness as a function of AWJM process parameters has direct application in predicting such component performance, and leads to a greater understanding of the means by which it can be minimized.

Several authors have developed process models for predicting surface roughness when using abrasive jets to machine brittle materials such as glass. For example, Jafar et al. [56] developed a numerical model for air abrasive jet machining (AJM) of micro-channels in borosilicate glass as a function of particle size, velocity, dose, impact angle and target material properties. The model simulated brittle erosion using two damage mechanisms: crater removal due to lateral crack formation and edge chipping. Roughness predictions were within a $29 \%$ of measured values. In another study by Jafar et al. [57] a similar model was used to predict roughness on borosilicate glass resulting from low-pressure abrasive slurry jet micro-machining (ASJM). These models 
cannot be used to predict roughness resulting from AJM of ductile materials, since the erosion mechanisms are cutting and ploughing rather than chipping.

Vikram and Babu [58] developed a surface roughness topography model for AWJ cuts in black granite (a brittle substrate) by superimposing irregularities produced by single abrasive particle impacts in order to generate an instantaneous surface topology. The irregularities were based on the predicted volume of material removed, the number of impacts and the crosssectional area of the abrasive particle. The instantaneous surface topography was then superimposed based on the amount of overlap of the jet (based on its depth of jet penetration and traverse speed) to generate the final effective topography. However, their model was only applied to brittle materials, and their surface roughness predictions neglected the effect of taper, required determination of empirical constants to resolve jet properties, and focused on the overall macro-mechanisms of AWJ material removal. Vikram and Babu's model predicted roughnesses on granite that were within $23 \%$ of those measured.

A number of empirical studies exist characterizing the roughness resulting from AWJM of ductile materials. The majority of these use regression analyses to fit a set of experimental observations to an empirical model. Examples of these include Jankovic et al. [59] for AWJM of 6060 aluminum alloy, Zohoor and Nourain [60] for hardox steel, Unde et al. [61] for carbonfiber reinforced plastics, Ramulu and Arola [62] for carbon-fiber/epoxy laminates, Azmir et al. [35] for aramid fiber reinforced polymer (FRP) composites, and Azmir and Ahsan [37] for glassfiber/epoxy laminates. Another empirical approach uses expert learning systems in an attempt to predict roughness trends based on experimental data. For example, Caydas and Hascalik [53] varied traverse speed, pressure, standoff distance (SOD), abrasive grit size and abrasive flow rate when machining 7075-T6 aluminum, and used artificial neural networks (ANN) to predict the resulting roughness trends. Shukla and Tambe [39] used a similar ANN approach to predict surface roughness of AWJM on Kevlar composites. Kok et al. [63] use genetic expression programming (GEP) to develop surface roughness models based on experimental results for AWJM of 7075 aluminum alloy. Cojbasic et al. [64] showed that the extreme learning machine (ELM) neural net methodology was more accurate than traditional ANN and GEP, when developing relations among AWJM operating conditions for surface roughness predictions. 
In summary, surface roughness is a key attribute in quantifying the quality of components cut using AWJM, but previous efforts to develop models for its prediction have been mostly empirical. The few analytical models that exist have focused on brittle materials and ignored changes in geometry brought about tapered kerfs. The present model is based on the fundamental principles of particle impact erosion on ductile materials, and predicts the surface roughness on the taper left after edge trimming of fiber-reinforced composite materials using $\mu \mathrm{AWJ}$ as a function of the process parameters, abrasive and substrate properties. The results of the model were compared with surface roughness measurements for two C-fiber composite materials machined under a wide range of conditions.

\subsection{Experiments}

\subsubsection{Abrasive Waterjet Machining Experiments}

AWJ edge-trimming cuts were performed on composite substrates in order to evaluate the predictive capabilities of the developed model. Two composite substrates were used in the experiments: a continuous carbon fiber ( $60 \%$ by wt.)/epoxy, uni-directional ply, laminate $[0 / 90 / 0]_{\mathrm{s}}$, and a randomly oriented chopped carbon fiber ( $50 \%$ by wt.)/vinyl sheet molding compound (SMC), with a nominal fiber length of $25 \mathrm{~mm}$. The material properties of the composite substrates shown in Table 2.1 were provided by the manufacturer (MAGNA Interiors, Concord, ON, Canada). 
Table 2.1 Composite material properties.

\begin{tabular}{|c|c|c|}
\hline Property & $\begin{array}{l}\text { Carbon fiber epoxy (CF-epoxy) - } \\
\text { single ply unidirectional }\end{array}$ & $\begin{array}{l}\text { Carbon fiber SMC (CF- } \\
\text { SMC) }\end{array}$ \\
\hline Matrix & Epoxy & Vinyl ester \\
\hline Reinforcement & Continuous carbon fiber & Chopped carbon fiber \\
\hline Fiber weight $\%$ & 60 & 50 \\
\hline Fiber orientation & Uni-directional & Random \\
\hline Thickness, $t,(\mathrm{~mm})$ & $1.2 \pm 0.017$ & $2.5 \pm 0.028$ \\
\hline $\begin{array}{l}\text { Specific gravity } \\
\left(\mathrm{g} / \mathrm{cm}^{3}\right)\end{array}$ & 1.53 (ASTM D792) & 1.41 (ASTM D792) \\
\hline $\begin{array}{l}\text { Tensile strength } \\
(\mathrm{MPa})\end{array}$ & 1277 (ASTM D3039) & 217 (ASTM D638) \\
\hline $\begin{array}{l}\text { Tensile modulus } \\
(\mathrm{GPa})\end{array}$ & 122 (ASTM D3039) & 28 (ASTM D638) \\
\hline
\end{tabular}

An OMAX 2626 Jet Machining Center (OMAX Corp. Kent, WA, USA), fitted with programmable nozzle movement allowing positional accuracy of $\pm 80 \mu \mathrm{m}$ over a $30 \mathrm{~cm}$ length, was used to perform the edge-trimming experiments on the two composites. The AWJM apparatus was fitted with a micro-nozzle having a $128 \mu \mathrm{m}$ diamond orifice and a $254 \mu \mathrm{m}$ mixing tube diameter, producing a $\sim 335 \mu \mathrm{m}$ footprint on a flat target surface at a SOD of $1 \mathrm{~mm}$ [65]. All experiments were conducted in air; i.e. with a jet and workpiece not submerged in water. The abrasive used in the experiments was $75 \mu \mathrm{m}$ garnet (Barton HPX®, Glen Falls, NY, USA) with a Mohs hardness of 7.5-8.5, a density of $\rho_{p}=4000 \mathrm{~kg} / \mathrm{m}^{3}$ [66]. Fig. 2.1 shows the distribution of the abrasive particle projected area diameters, based on sieve analysis data provided by the supplier. 


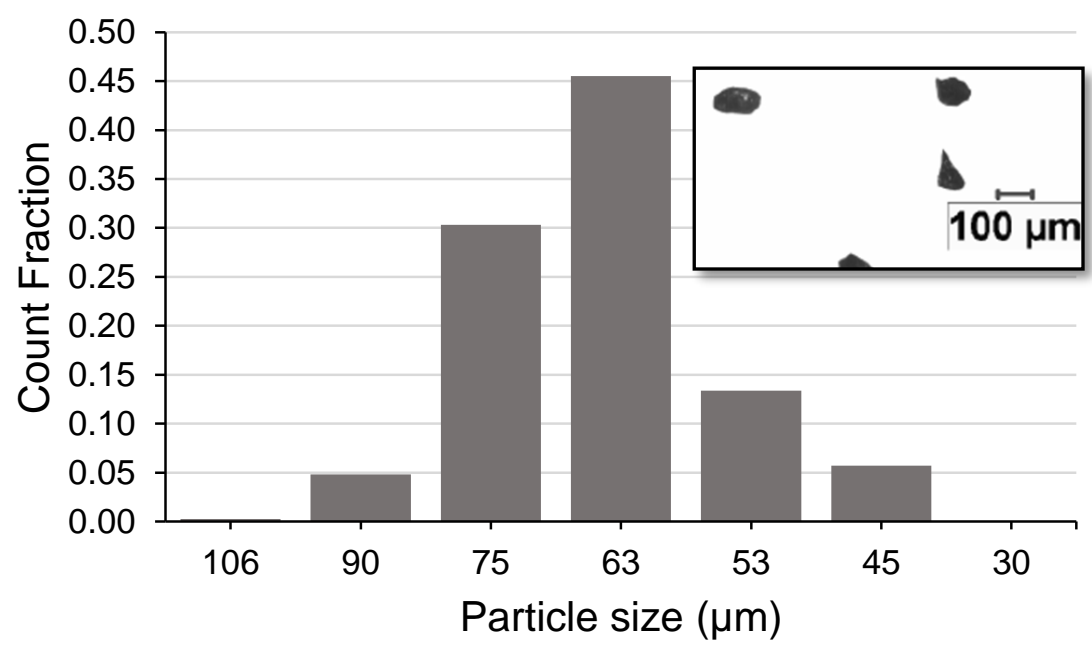

Fig. 2.1 Abrasive particle size distribution (projected area diameter). Inset micrograph of particle shape.

During AWJM cutting, particles can ricochet from one side of the cut to the other (Fig. 2.2a and Fig. 2.3). Trimming cuts were made to eliminate the effects of secondary particle strikes, and focus solely on the effects of the incident particles. The nozzle path was adjusted to perform trimming operations over a $10 \mathrm{~cm}$ length in which the cut edge of a slot was trimmed by a second pass of the nozzle that had been shifted half the jet width $(\sim 162.5 \mu \mathrm{m}$; positioning accuracy of AWJ apparatus was $12.5 \mu \mathrm{m} / \mathrm{step}$ ) (Fig. 2.3). It has been shown in the literature that surface roughness commonly increases with increasing depth of AWJM cuts due to drag forces on the particles and an increased local particle impact angle due to the bending of the jet at the cutting front [58]. In general, decreasing the traverse speed results in an increase in cut quality and a reduction in the variation of roughness and waviness along the kerf ( $z$ direction in Fig. 2.3) [67].

Previous investigators [53] [29, 68, 69] have found that three cutting regions exist along an AWJ kerf: an initial damage region along the entrance of the kerf, a smooth cutting region near the top of the cut, and a rough cutting region near the bottom of the kerf. However, with proper selection of the operating conditions, the cut quality can be improved such that the smooth cutting region can be effectively extended through the entire thickness of the cut [2], with little variation of the surface roughness with depth. Therefore, in its current state, the proposed model 
is only capable of predicting the surface roughness during steady-state cutting for such cases, i.e. when there is a constant roughness along the depth of the kerf.

The traverse speed was chosen to ensure that the trimming cuts were within this steady state cutting regime (i.e. surface roughness was constant along the depth of the kerf in the $z$-direction of Fig. 2.3). The trimming cuts used in the experiments ensured that the surface was cut at a steady state, since the drag on the incoming jet was unaffected by the confines of a slot and secondary particle strikes from sidewall to sidewall were eliminated when the slot was wide enough, as illustrated in Fig. 2.2. The influence of cutting front curvature, i.e. perpendicular to the direction of traverse associated with AWJM was not considered in the current model. Ahmed et al. [70] have shown that even for a zero trim width (i.e., a through cut), virtually all the particles strike the sidewalls at very shallow angles, indicating very few particles ricochet onto the sidewalls from the curved cutting front. Moreover, the curvature of the cutting front at the leading edge of the jet was not considered to influence the erosion of the sidewalls and was thus not included in the current model.

The measurements of the arithmetic average roughness $\left(R_{a}\right)$ in the $z$-direction along the kerf of each trim are shown in Fig. 2.2b as a function of the trim width defined in Fig. 2.2a. When the trim width is zero (a through-cut slot), $R_{a}$ is relatively high due to the second strike of particles as illustrated in the figure. As the trim width increased, the number of secondary particle strikes impacting the trimmed kerf decreased, reaching a minimum at about $150 \mu \mathrm{m}$ when there are relatively few second strikes. After this point, the roughness begins to increase as the jet position was moved from trimming and back into the through cutting regime. Therefore, subsequent experiments were conducted with a trim width equal to half the jet footprint $(162 \mu \mathrm{m})$ to minimize particle second strikes and simplify the roughness modelling along the kerf. 

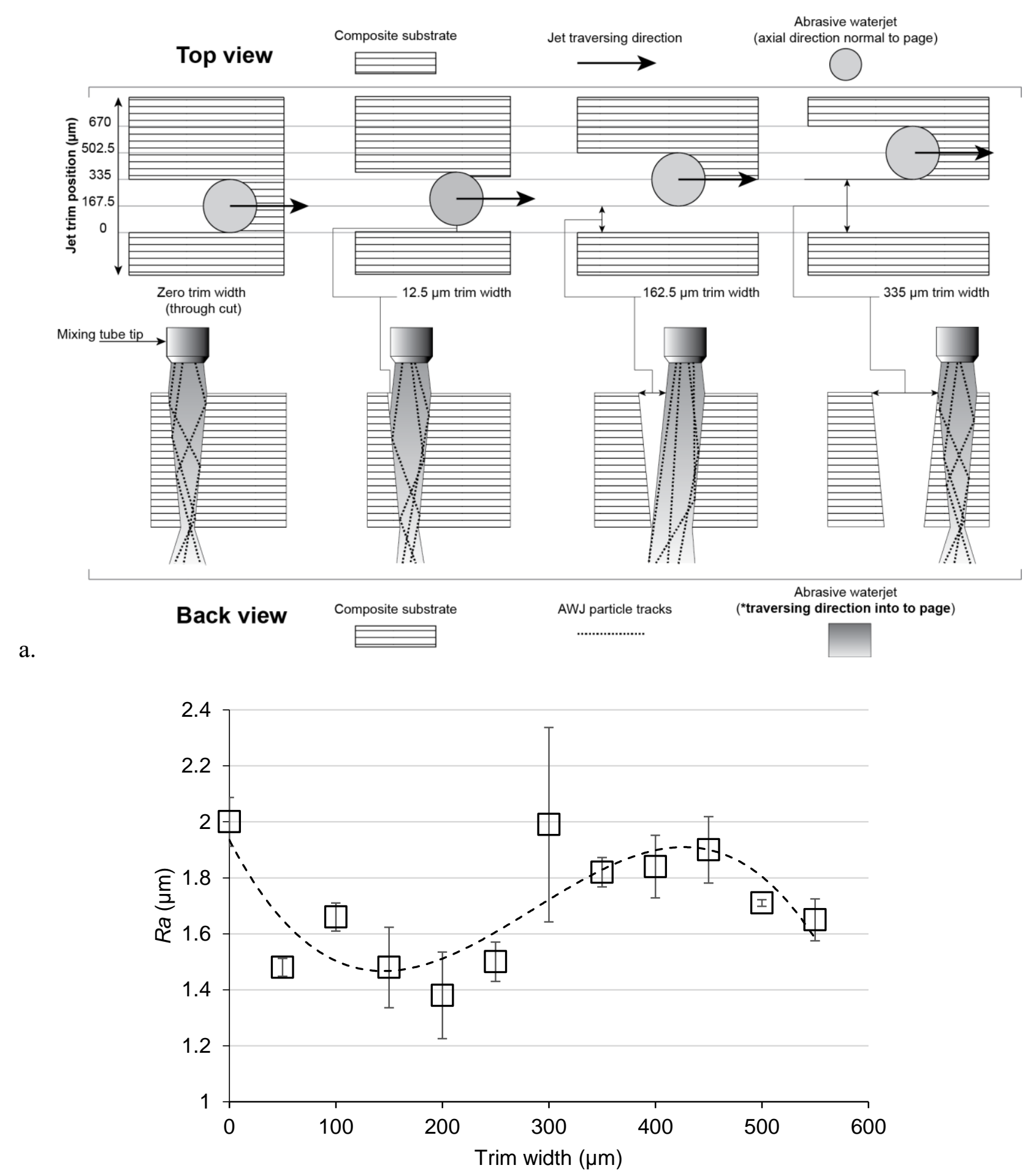

b.

Fig. 2.2 a. Schematic representation of trimming at four different trim widths (note that in the back view of part a, the jet is traversing into the page). b. The effect of trimming width on kerf roughness. Material: CF-SMC, pressure: $225 \mathrm{MPa}$, SOD: $1 \mathrm{~mm}, \dot{m}_{a}=0.2 \mathrm{~g} / \mathrm{s}$, abrasive: 75 micro garnet. Error bars show the variation in surface roughness among three specimens. 
The specimens were clamped along their edges to a rigid steel grate with $40 \mathrm{~mm}$ openings.

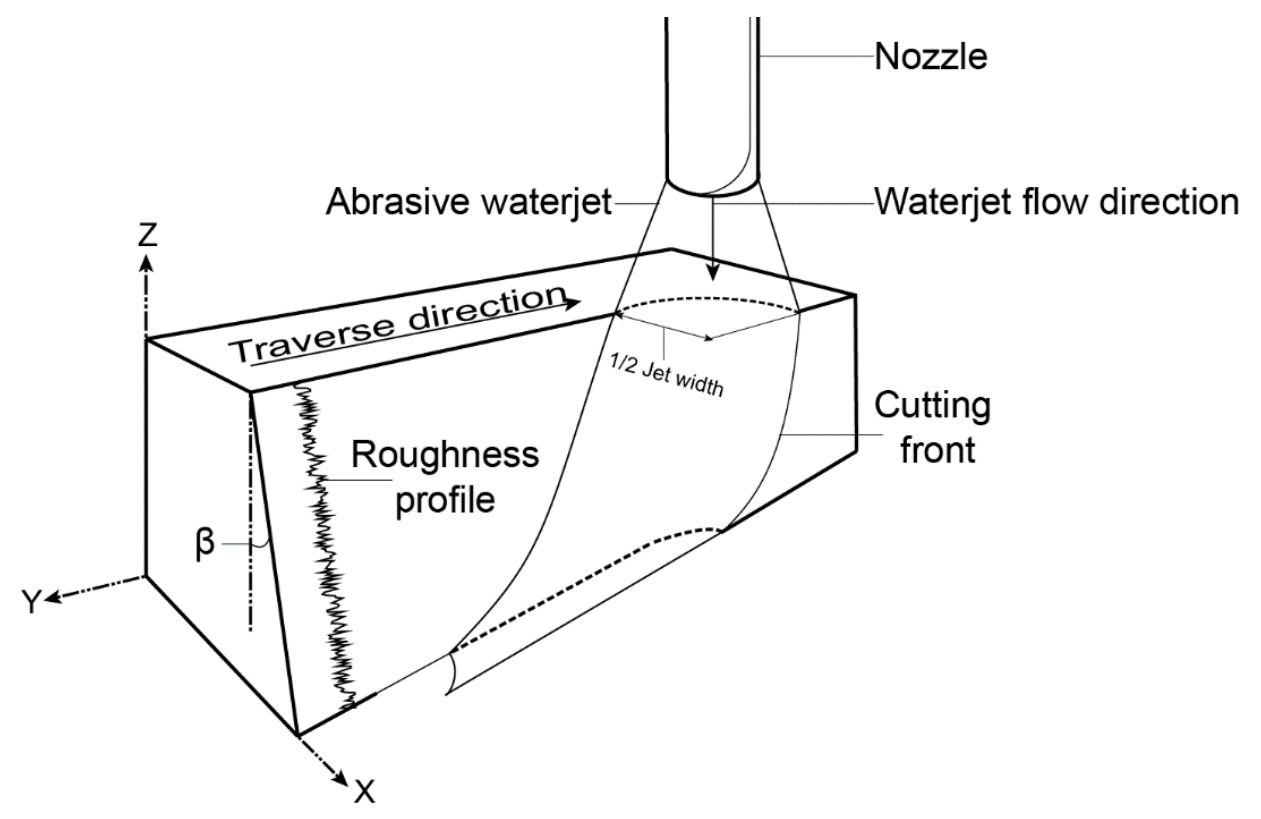

Fig. 2.3 Schematic of AWJM edge trimming and the location of the profilometer scan used to measure $R_{a}$ on the kerf of the trimmed edge.

The experiments were performed according to a two-factor (abrasive velocity, average specific surface dose, $D_{s, \text { ave }}$ ), three-level design of experiments, shown in Table 2.2. Abrasive velocity and flux are fundamental parameters governing AWJ erosion since they quantify the number of erodent particles striking the surface and their kinetic energy. They are in turn functions of the operating parameters for the specific AWJ machine being used; e.g. pressure, nozzle size, abrasive flow rate, standoff distance and traverse speed. The abrasive velocity was estimated using the model developed by Narayanan et al. [71], which has been shown to predict axial particle velocities within 5\%. The abrasive velocities were chosen within the limitations of the pump and the cutting requirements of the composite materials. 
The average specific surface dose, i.e. the mass of particles that would be delivered per unit area of the substrate surface in a single traverse is given by

$$
D_{s, a v e}=\frac{\dot{m}_{a} \cdot L_{t}}{U \cdot A}=\frac{\dot{m}_{a}}{U \cdot D_{f}}
$$

where $\dot{m}_{a}$ is the abrasive mass flow rate (held constant at $\sim 0.5 \mathrm{~g} / \mathrm{s} \pm 0.05 \mathrm{~g} / \mathrm{s}$ ), $U$ is the nozzle traverse speed $(\mathrm{m} / \mathrm{s})$, and the area of the target exposed to the abrasive stream is $A=L_{t} \cdot D_{f}$, where $L_{t}$ is the length of the nozzle traverse and $D_{f}$ is the diameter of the abrasive waterjet footprint. The footprint diameter was determined using the work of Haghbin et al. [72], and was held relatively constant $\left(D_{f} \approx 335 \mu \mathrm{m}\right)$, at a stand-off distance of $1 \mathrm{~mm}$ throughout all experiments. The average specific dose $\left(\mathrm{kg} / \mathrm{m}^{2}\right)$ of particles striking the kerf of the edge trim is then given by

$$
D_{p}=D_{s, a v e} \cdot \sin (\beta)
$$

where $\beta$ is the measured taper angle shown in Fig. 2.3.

All experiments were performed under typical AWJM operation conditions [72-74] at a jet angle of $90^{\circ}$, and an input water temperature of $12^{\circ} \mathrm{C}$. The conditions are shown in Table 2.2. 
Table 2.2 Experimental conditions, measured taper angles (Fig. 2.3) \pm 1 standard deviation and resulting average specific dose on the kerf area $\left(D_{p}\right)$.

\begin{tabular}{|c|c|c|c|c|c|c|c|}
\hline ID & $\begin{array}{l}\text { Abrasive } \\
\text { Velocity } \\
(\mathbf{m} / \mathbf{s})\end{array}$ & $D_{s, \text { ave }}\left(\mathrm{kg} / \mathrm{m}^{2}\right)$ & $\begin{array}{l}\text { Pressure } \\
\text { (MPa) }\end{array}$ & $U(\mathrm{~cm} / \mathrm{s})$ & $\begin{array}{l}\text { Target } \\
\text { Material }\end{array}$ & $\beta\left({ }^{\circ}\right)$ & $D_{p}\left(\mathrm{~kg} / \mathrm{m}^{2}\right)$ \\
\hline 1 & 550 & 36.2 & 212 & 4.12 & CF-epoxy & $12 \pm 0.5$ & 7.29 \\
\hline 2 & 550 & 80.4 & 212 & 1.85 & “" & $8 \pm 0.5$ & 11.85 \\
\hline 3 & 550 & 442.2 & 212 & 0.33 & “ & $6 \pm 1.3$ & 48.70 \\
\hline 4 & 600 & 36.2 & 250 & 4.12 & “ & $8 \pm 0.3$ & 5.18 \\
\hline 5 & 600 & 80.4 & 250 & 1.85 & “ & $7 \pm 1.3$ & 9.70 \\
\hline 6 & 600 & 442.2 & 250 & 0.33 & “ & $4 \pm 0.6$ & 27.30 \\
\hline 7 & 650 & 36.1 & 292 & 4.12 & “ & $4 \pm 1.4$ & 2.49 \\
\hline 8 & 650 & 80.4 & 292 & 1.85 & “ & $5 \pm 0.2$ & 7.05 \\
\hline 9 & 650 & 442.2 & 292 & 0.33 & “ & $3 \pm 0.1$ & 22.46 \\
\hline 10 & 550 & 110.5 & 212 & 1.35 & CF-SMC & $11 \pm 0.2$ & 20.87 \\
\hline 11 & 550 & 201.0 & 212 & 0.74 & “ & $7 \pm 0.3$ & 23.47 \\
\hline 12 & 550 & 602.9 & 212 & 0.24 & “ & $3 \pm 0.2$ & 31.36 \\
\hline 13 & 600 & 110.5 & 250 & 1.35 & “ & $10 \pm 0.6$ & 19.51 \\
\hline 14 & 600 & 201.0 & 250 & 0.74 & “ & $9 \pm 0.2$ & 30.38 \\
\hline 15 & 600 & 602.9 & 250 & 0.25 & “ & $4 \pm 0.3$ & 40.75 \\
\hline 16 & 650 & 110.5 & 292 & 1.35 & “ & $8 \pm 0.1$ & 15.55 \\
\hline 17 & 650 & 201.0 & 292 & 0.74 & “ & $6 \pm 0.1$ & 20.27 \\
\hline 18 & 650 & 602.9 & 292 & 0.25 & “ & $5 \pm 0.3$ & 50.38 \\
\hline
\end{tabular}

\subsubsection{Dynamic Hardness Measurement}

The rigid-plastic model used in modelling the craters left by individual particle impacts (described in Section 2.3.2) required the input of dynamic hardness $p_{d}$, a measure of the resistance of the target material to dynamic indentation. It is defined as the average force per unit projected area resisting the impact, and can be approximated as [75-78]

$$
p_{d}=\frac{0.5 \cdot m \cdot V_{p}^{2}}{V}
$$

where $m$ and $V_{p}$ are the impacting spherical particle mass and velocity, respectively, and $V$ is the volume of the resulting spherical crater [77]

$$
V=\left(\frac{\pi}{6}\right)\left(R_{p}-\sqrt{R_{p}^{2}-r^{2}}\right)\left(3 r^{2}+\left(R_{p}-\sqrt{R_{p}^{2}-r^{2}}\right)^{2}\right)
$$


where $R_{p}$ is the nominal particle radius and $r$ is the crater radius.

The dynamic hardness of the two target materials was inferred from the measured volume of craters left by the impact of 79.2 $\pm 12.6 \mu \mathrm{m}$ steel shot (Vulkan-INOX, Germany) whose size distribution is shown in Fig. 2.4. The crater circular diameters were measured using Clemex PE Vision 5.0 image analysis software (Clemex Technologies Inc. Longueuil, QC, Canada), based on micrographs of the steel shot obtained using a Leica DM2500 M microscope (Leica Microsystems, Buffalo Grove, IL, USA) with magnification capabilities of $25 \mathrm{x}-1000 \mathrm{x}$ using a reflected light source. In order to produce widely-spaced single impact craters, the particle jet was directed through a dual-disk anemometer (DDA) [79] spinning at 23,000 RPM. The steel shot waterjet was produced using the same setup described in Section 2.2.1 at a pressure of 134 $\mathrm{MPa}$, stand-off distance of $12.4 \mathrm{~mm}$, and a steel shot flow rate of $3.8 \mathrm{~g} / \mathrm{min}$. Twenty crater radius measurements were made for each of the CF-epoxy and CF-SMC materials.

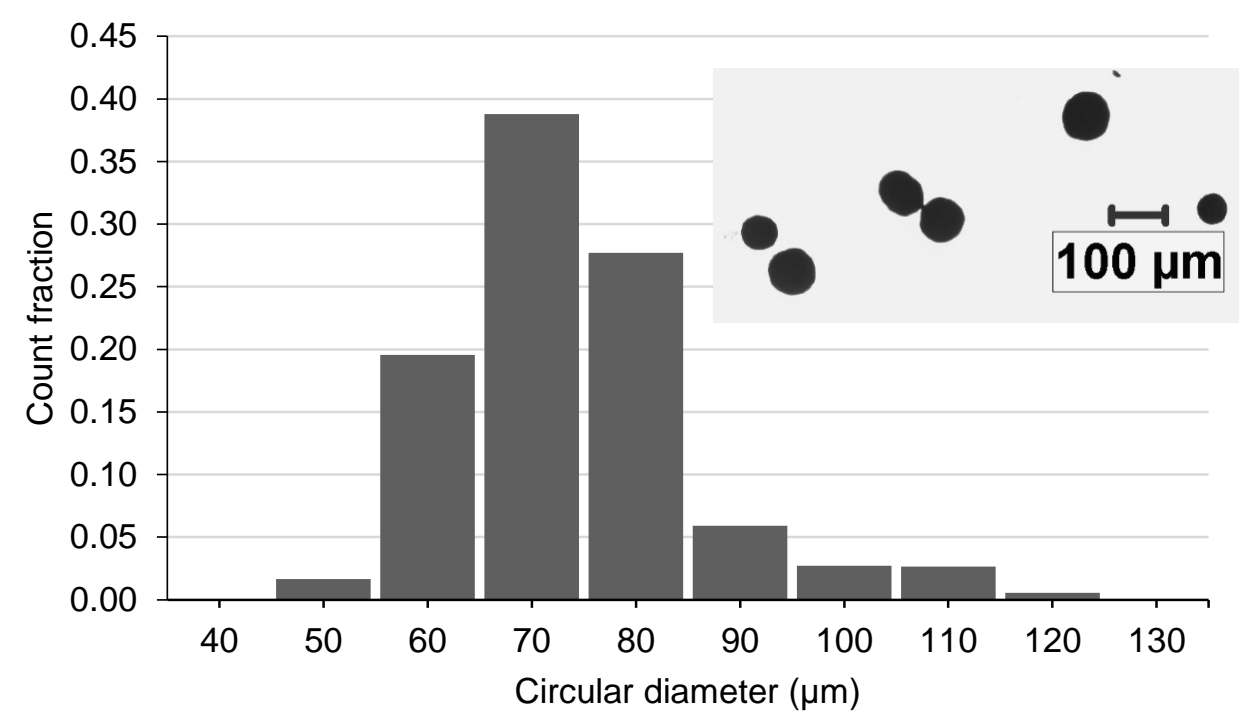

Fig. 2.4 Size distribution of steel shot used to measure target dynamic hardness. The inset shows micrograph of particles.

The average mass of each steel shot particle was calculated as $2.05 \mu \mathrm{g}$ based on the average particle diameter of $79.2 \mu \mathrm{m}$, and the average particle velocity was $436 \mathrm{~m} / \mathrm{s}$ using the model developed by Narayanan et al. [71]. 


\subsubsection{Surface Roughness and Taper Angle Measurements}

The surface roughness measured vertically along the walls of the kerfs (Fig. 2.5) was obtained using an optical profilometer having a lateral resolution of $426 \mathrm{~nm}$ and depth resolution of $16 \mathrm{~nm}$ (ST400, Nanovea Inc., CA, USA). The arithmetic mean roughness $\left(R_{a}\right)$ was used to assess the surface topography in accordance with ISO 4287. Due to the relatively thin crosssections, the evaluation length (1.2 and $2.5 \mathrm{~mm}$ for CF-epoxy and CF-SMC, respectively) spanned the entire kerf, with a cut-off wavelength equivalent to $20 \%$ of the evaluation length (240 and $500 \mu \mathrm{m}$ for CF-epoxy and CF-SMC, respectively). The roughness profile was evaluated using Mountain 6 software (Digital Surf, France). The taper angle (Fig. 2.5) was a measured characteristic of the cut under the given machine parameters (Table 2.2) since there is currently no analytical model that can accurately predict it.

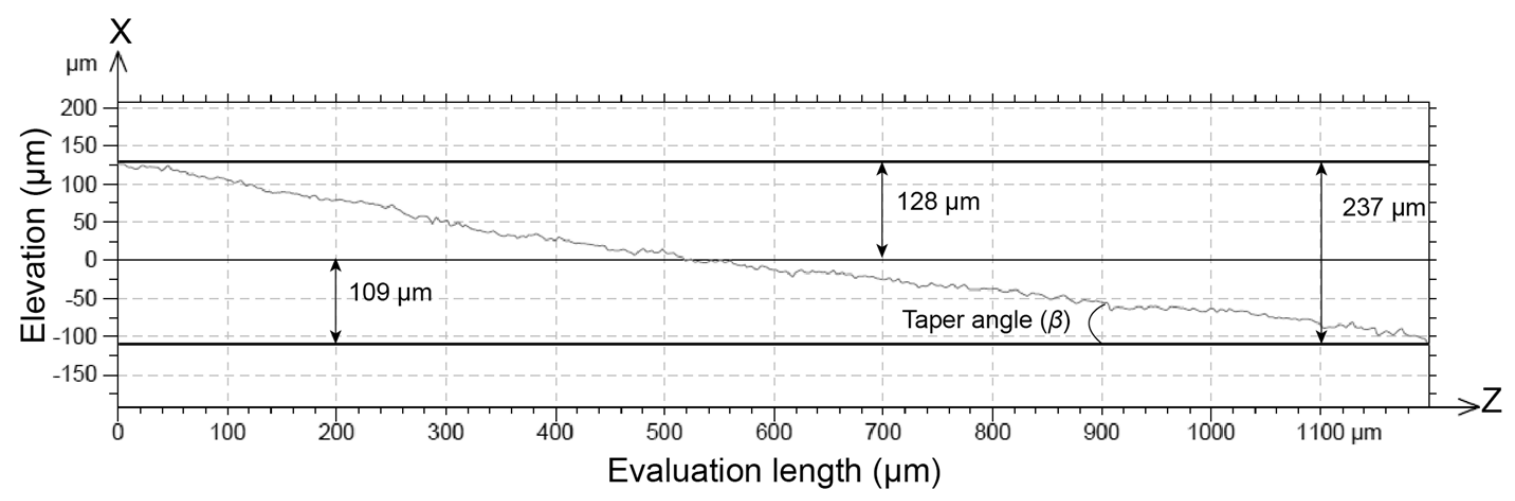

Fig. 2.5 Typical measured taper profile showing measured angle for specimen ID 1 in Table 2.2. Coordinates defined as in Fig. 2.3. 


\subsection{Modelling}

Two and three dimensional models were developed to predict the sidewall surface roughness (Fig. 2.3) of an AWJ trimming cut as a function of the AWJ operating conditions of pressure, traverse speed, abrasive flow rate, abrasive properties and composite material properties.

\subsubsection{D Model Overview}

The model superimposed two dimensional (2D) craters resulting from individual particle impacts along the side of the kerf in order to produce a 2D profile of the sidewall. This approach is consistent with Zhou et al. [80] who hypothesized that the surface generated by an AWJ in the smooth cutting region is formed by the superposition of micro-dimples created by individual abrasive impacts. Fig. 2.6 shows the program structure as implemented in MATLAB R2014 (Mathworks, MA, USA) and Mathcad 14 (PTC, MA, USA) mathematical software.

In the first step (block A in Fig. 2.6), individual 2D craters were generated using the rigidplastic impact model of Papini and Spelt $[18,19]$ which assumes the impact of rigid rhomboid particles on a fully-plastic ductile target. This model, described in Section 2.3.2, takes as inputs the abrasive particle size, angularity and orientation (Section 2.3.2.2), abrasive density, impact angle (Section 2.3.2.4) and the target dynamic hardness, and outputs the coordinates of an impact crater profile. As described in Section 2.3.2.3, the impact angle includes the effect of the taper angle and the random component of radial velocity in the jet (Section 2.3.2.1). The program repeatedly generated single-particle craters until there were sufficient craters to span the kerf length (Fig. 2.3) when positioned consecutively adjacent to each other with no overlap (block B in Fig. 2.6). The kerf length was approximated as the thickness of the target material, which was justified by the relatively small kerf angles. This was then repeated, each time generating a profile of contiguous, non-overlapping craters that were then superimposed on the previous profile about the midline to create a multi-particle impact profile (block C in Fig. 2.6; described in Section 2.3.4). Successive profiles were superimposed until the model reached a constant steady-state surface roughness. 


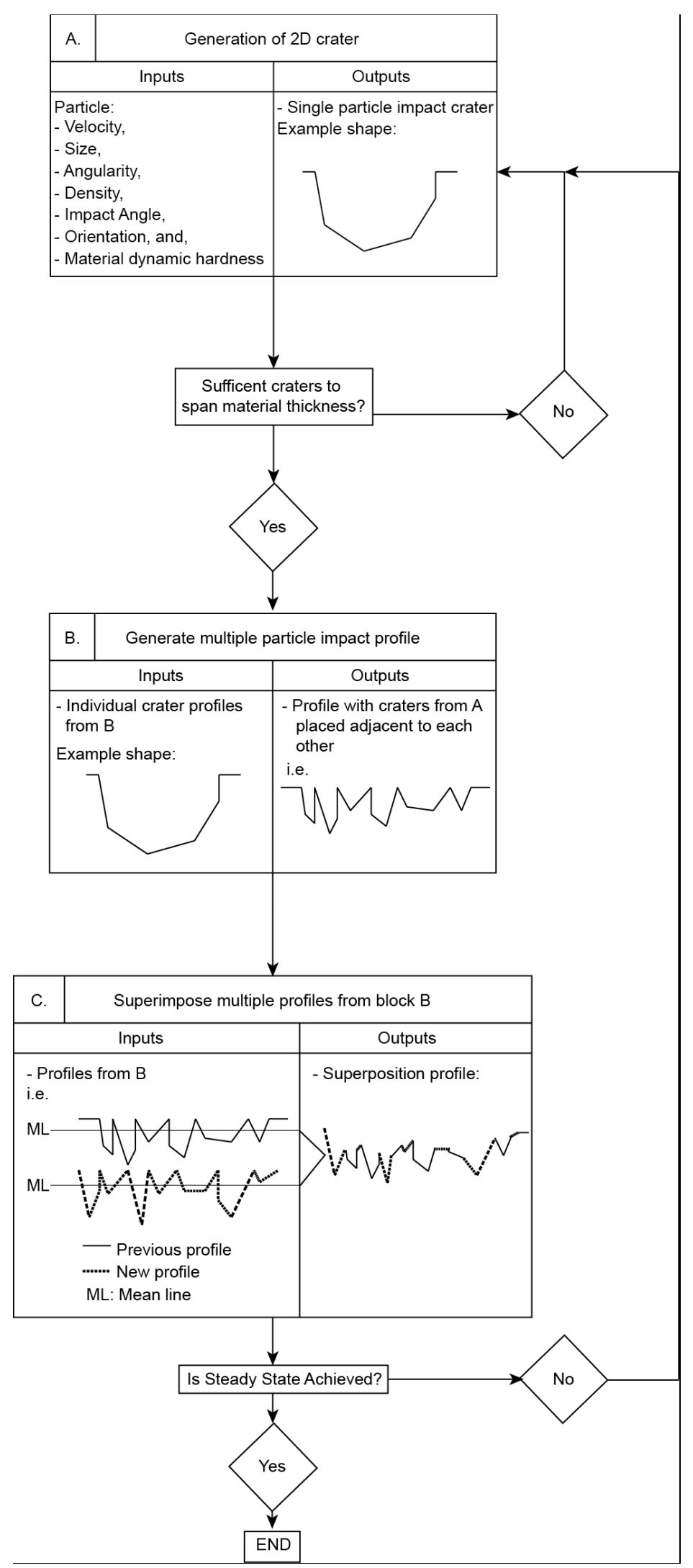

Fig. 2.6 Structure of surface roughness model. 


\subsubsection{Rigid-Plastic Model to Obtain Individual Particle Craters (Block A)}

A detailed description of the model used in block A of Fig. 2.6 can be found in the work of Papini and Spelt [18, 19]. Briefly, it assumes a non-deforming rhomboid (Fig. 2.7) 'rigid' particle impacting a fully-plastic target material which resists the indentation with an instantaneous contact force equal to the product of the constant dynamic hardness, $p_{d}$, and the instantaneous contact area. The resulting equations of motion describing the particles as they plough or cut through the target must be integrated numerically because the contact area, and thus the magnitude and direction of the contact force, changes throughout the impact as the particles translate and rotate. The inputs are the particle velocity $\left(V_{i}\right)$, size $(h)$, angularity $(A)$, orientation $(\theta)$, impact angle $(\alpha)$, and the density and dynamic hardness, $p_{d}$, of the target (Fig. 2.7). The output is the coordinates of a crater resulting from a single particle impact.

\subsubsection{Particle Velocity}

The impact particle velocity was approximated using the model developed by Narayanan et al. [71]. The model was used with the inputs shown in Table 2.3. 
Table 2.3 AWJ velocity model inputs.

\begin{tabular}{ll} 
Parameter & Value \\
\hline Orifice diameter $(\mu \mathrm{m})$ & 127 \\
Mixing tube length $(\mathrm{mm})$ & 27.9 \\
Mixing tube diameter $(\mu \mathrm{m})$ & 254 \\
Abrasive feed pipe length $(\mathrm{m})$ & 0.50 \\
Abrasive feed port diameter $(\mathrm{mm})$ & 1.52 \\
Water density $\left(\mathrm{kg} / \mathrm{m}^{3}\right)$ & 1000 \\
Water viscosity $(\mathrm{kg} / \mathrm{m} \mathrm{s})$ & 0.00100 \\
Air density $\left(\mathrm{kg} / \mathrm{m}^{3}\right)$ & 1.17 \\
Air viscosity $\left(\mathrm{kg} / \mathrm{m} \mathrm{s}^{3}\right)$ & $1.8 \times 10^{-5}$ \\
Pressure $(\mathrm{MPa})$ & As shown in Table 2.2 \\
Abrasive flow rate $(\mathrm{g} / \mathrm{s})$ & 0.50 \\
Abrasive density $\left(\mathrm{kg} / \mathrm{m}^{3}\right)$ & 4000 \\
Mixing chamber diameter $(\mathrm{mm})$ & 6.32 \\
Mixing chamber length $(\mathrm{mm})$ & 5.99 \\
Ambient pressure $(\mathrm{kPa})$ & 101 \\
Ambient temperature $(\mathrm{K})$ & 300 \\
Discharge coefficient & 0.65 \\
Mean abrasive diameter $(\mu \mathrm{m})$ & 70 \\
\hline
\end{tabular}

The standoff distance of $1 \mathrm{~mm}$ ensured that particle deceleration was negligible since the core of the jet extended to the target surface [81]. The radial component of the particle velocity was estimated based on the work of Balz et al. [82] who showed that the radial particle velocity in AWJ ranges from $0-4 \%$ of the axial velocity.

\subsubsection{Particle Size, Angularity and Orientation}

Similar to the particle embedment studies of Getu et al. [77], the dimensions $(D, d)$ and angularity $\left(A_{1}, A_{2}\right)$ of the idealized rhomboids used to model the actual particles (Fig. 2.7) were based on the particle size (Fig. 2.1) and aspect ratio (AR) taken from the measured distributions. Specification of the particle thickness was not required since the model was two-dimensional with particle mass, moment of inertia, and the volume of the crater expressed on a per-unitthickness basis. 


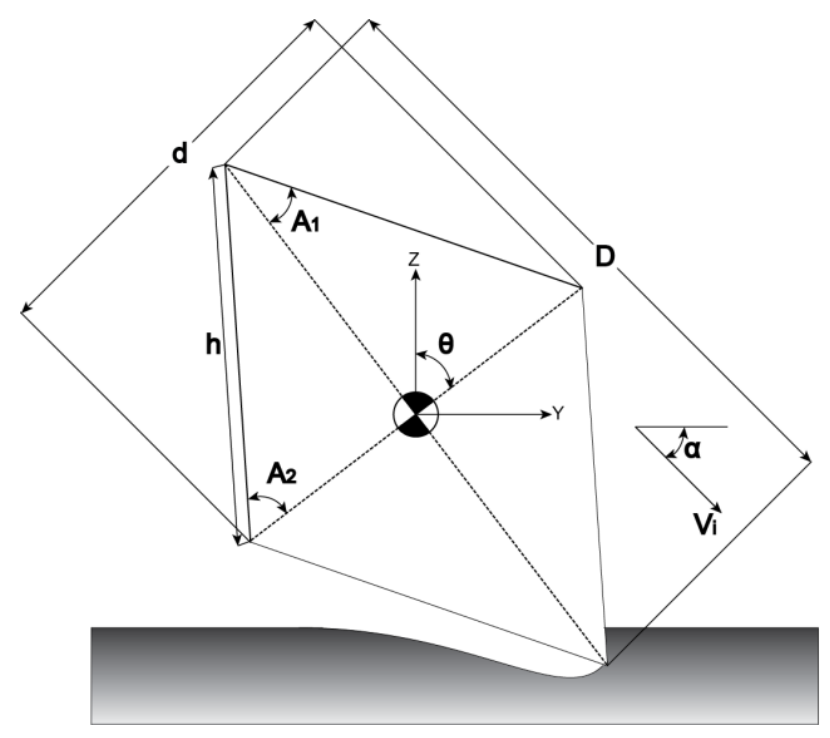

Fig. 2.7 Schematic of a model particle as it plows a crater in a ductile substrate according to the rigid-plastic model $[18,19]$. $V_{i}$ - impact velocity, $h$ - idealized rhomboid length, $A_{l}$ and $A_{2}$ particle angularity, $\theta$ - particle orientation, $\alpha$ - impact angle, $D$ - particle length, $d$ - particle width.

The $A R$ values were measured using photographs of the abrasive particles taken with the same microscope setup described in Section 2.2.2. The abrasive had a mean aspect ratio of $A R=1.63$ with a standard deviation of \pm 0.403 , which implies

$$
\begin{aligned}
& A_{1}=\tan ^{-1}(A R)=58^{\circ} \mp 22^{\circ} \\
& A_{2}=90^{\circ}-A_{1}
\end{aligned}
$$

The values of $A_{1}$, and as a result, $A_{2}$, were randomly assigned to particles within the bounds of the standard deviation in equation (2.5). The orientation of the particle upon impact was randomly varied from $0^{\circ}-360^{\circ}$ which meant that a given particle could either impact with an angularity corresponding to $A_{1}$ or $A_{2}$, depending on which vertex was closest to the target.

\subsubsection{Impact Angle}

The impact angle was the vector sum of the taper angle $(\beta)$ (Fig. 2.5 and Fig. 2.8) and resultant particle impact velocity vector angle, as shown in Fig. 2.8. Measurements of the kerf taper angles (Table 2.2) on the trimmed edges revealed that the kerf could be modelled 
approximately as a straight line [83], and that its slope $(\beta)$ depended strongly on the traverse speed, with a higher traverse speed leading to a shallower slope. This is consistent with the observations of Shanmugam and Masood [84] for AWJM in layered composites, Azmir et al. [85] for fiber reinforced plastics, Shanmugam et al. [86] for alumina ceramics, Hascalik et al. [87] for titanium alloy, and Hlavac et al. [88] for steels. The taper angle varied with the operating parameters, mainly the traverse speed since it affected the dose on the trimmed edge, and primarily controlled the particle impact angle in the analytical model.

As mentioned in Section 2.3.2.1, the radial particle velocities were randomly assigned between 0 and $4 \%$ of their axial velocity, based on the results of Balz et al. [82].

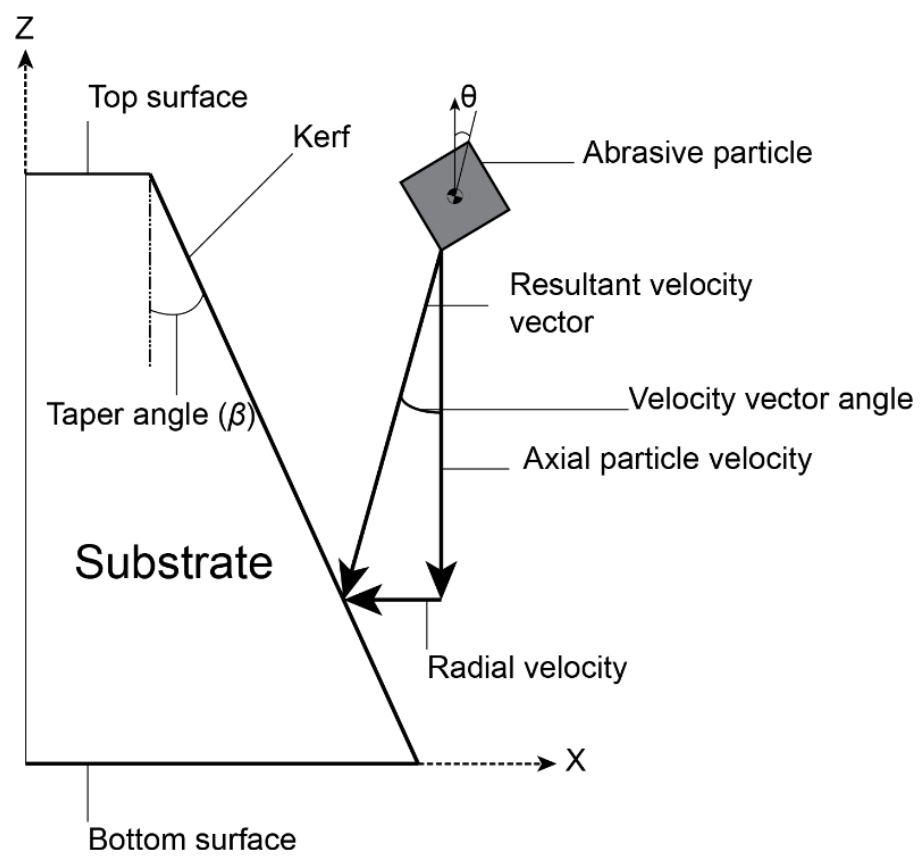

Fig. 2.8 Impact of an idealized rhomboid striking the kerf of the trimmed edge. The direction of nozzle traverse is normal to the plane of the figure. 


\subsubsection{Multiple Particle Impact Profile (Block B)}

Typical single-particle impact craters obtained from the rigid-plastic model were generally quite long and shallow in both target materials, as illustrated in Fig. 2.9 for the CF-epoxy. The model predicted the ploughing of such craters as the particle translated and rotated in contact with the ductile target $[18,19]$.

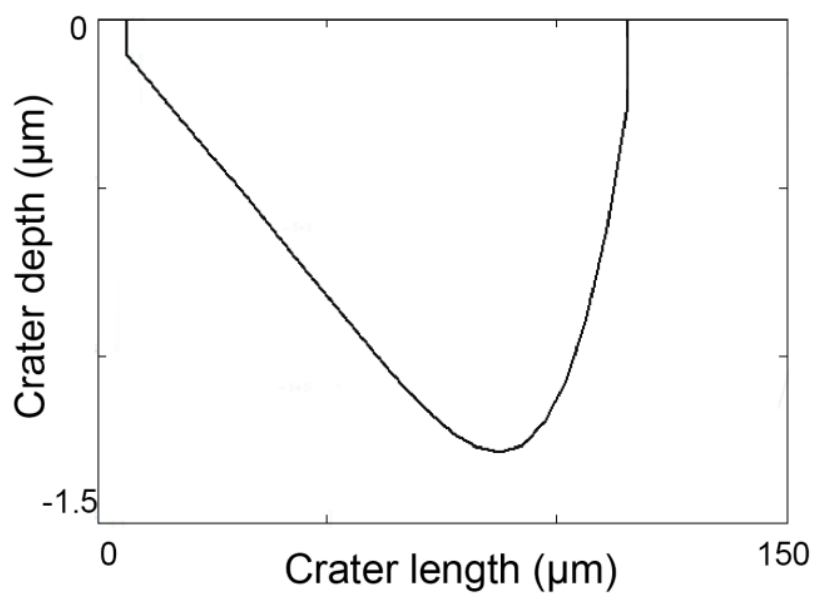

Fig. 2.9 Predicted single-particle impact crater on CF-epoxy substrate for a particle with $A_{l}=56.4^{\circ}, A_{2}=33.6^{\circ}, \rho_{p}=4000 \mathrm{~kg} / \mathrm{m}^{3}, \alpha=0.97^{\circ}, \theta=7.6^{\circ}, V_{i}=650 \mathrm{~m} / \mathrm{s}$. Note the large difference in depth and length scales.

Fig. 2.10 illustrates the concatenation of single-impact model craters over the length of the kerf for the conditions of ID 8 in Table 2.2. For simplicity of notation, the same $x z$ coordinates are used as in Fig. 2.3 and Fig. 2.8, even though they strictly should be rotated through the small angle $\beta$ in the counter-clockwise direction so that $z$ is directed along the surface of the kerf. 


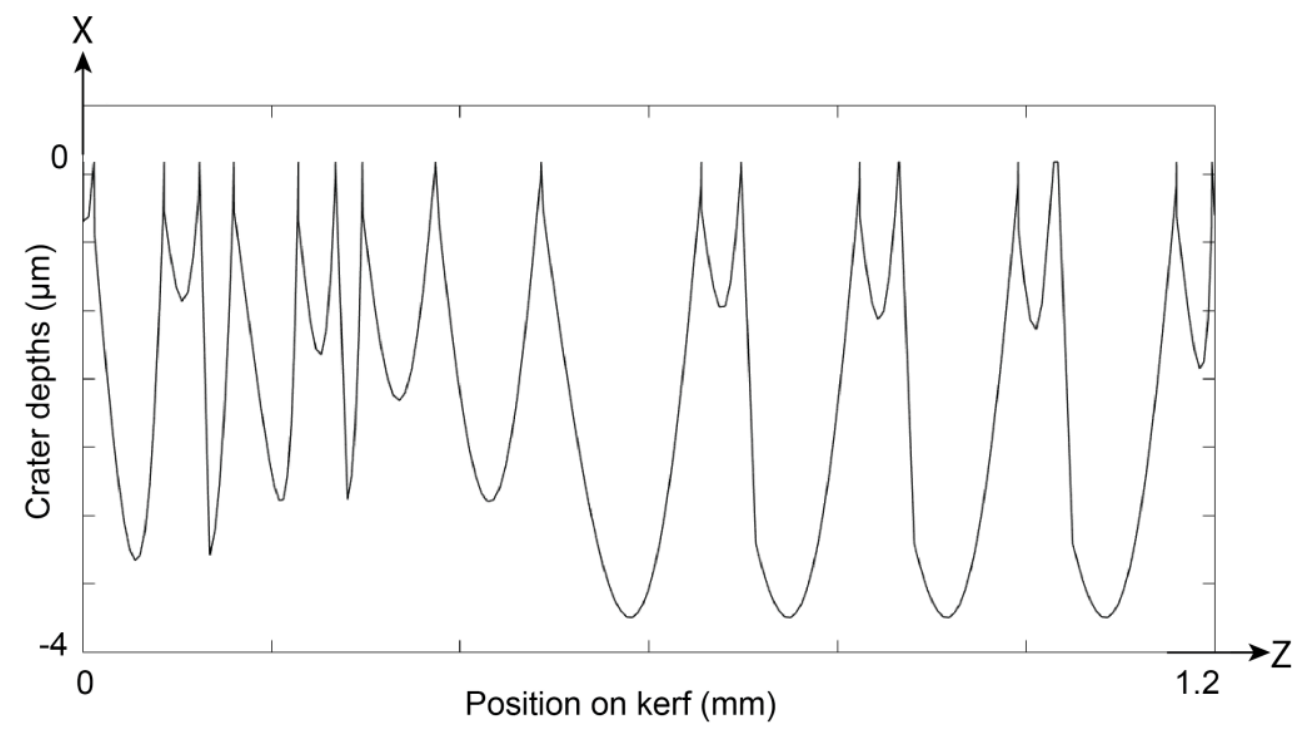

Fig. 2.10 Roughness profile of 17 contiguous single-particle impact craters for the CF-epoxy substrate for the conditions of ID 8 in Table 2.2.

\subsubsection{Method to Superimpose Multiple Profiles (Block C)}

Two approaches to superimposing successive profiles were investigated. In the first, simple additive superposition of the profiles was used, under the assumption that the depth of any point on the profile at any given iteration would be proportional to the simple sum of the erosive energy incident at that point. However, as shown in Fig. 2.11, this resulted in $R a$ predictions that rapidly increased to values that were far higher than measured, and which never reached steady state. Such a scheme overestimates the erosion of the valleys, because, for the mostly low angle, glancing kerf impacts that occur during edge trimming, the peaks tend to protect the valleys, but this shielding effect is not simulated in the simple additive superposition model. To avoid this, an alternate technique for superimposing profiles was utilized which allowed peaks to be eroded, but valleys to be preserved unless directly exposed to an impact. The technique is analogous to that used by Jafar et al. [56] in the simulation of the smoothing of glass surfaces due to the edge chipping of peaks when impacted by particles at oblique incidence. 


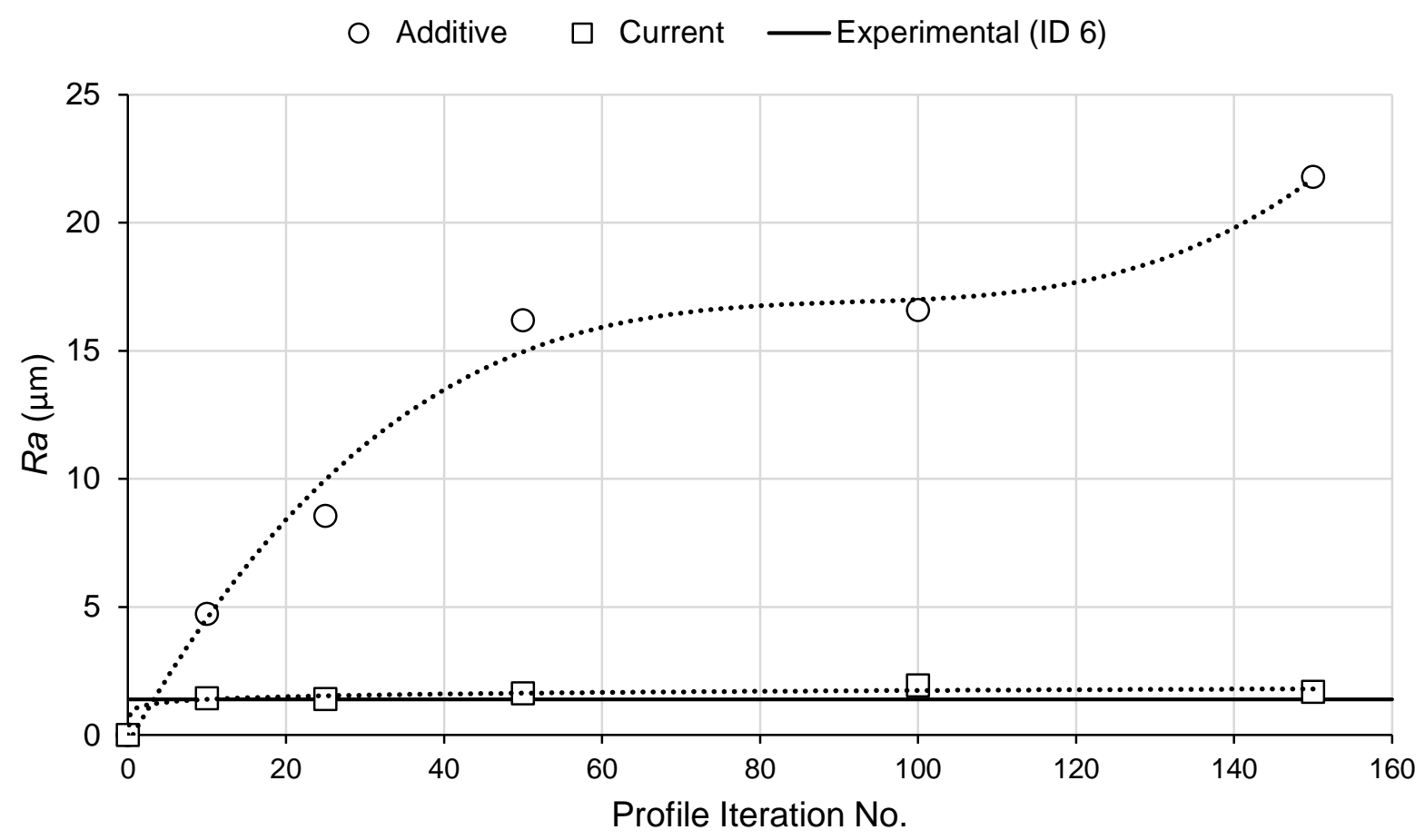

Fig. 2.11 Comparison of current method vs. additive superposition relative to iteration number for specimen ID 6 in Table 2.2.

Multiple profiles were superimposed as illustrated in Fig. 2.12 using the second approach. Successive roughness profiles were aligned about the elevation of their mean lines, $M_{L}$, which for equally spaced profile data points was given by

$$
M_{L}=\frac{\sum_{i=1}^{n} x_{i}}{n}
$$

where $x$ is the depth of the data point at point $i$ along the kerf and $n$ is the number of data points. 


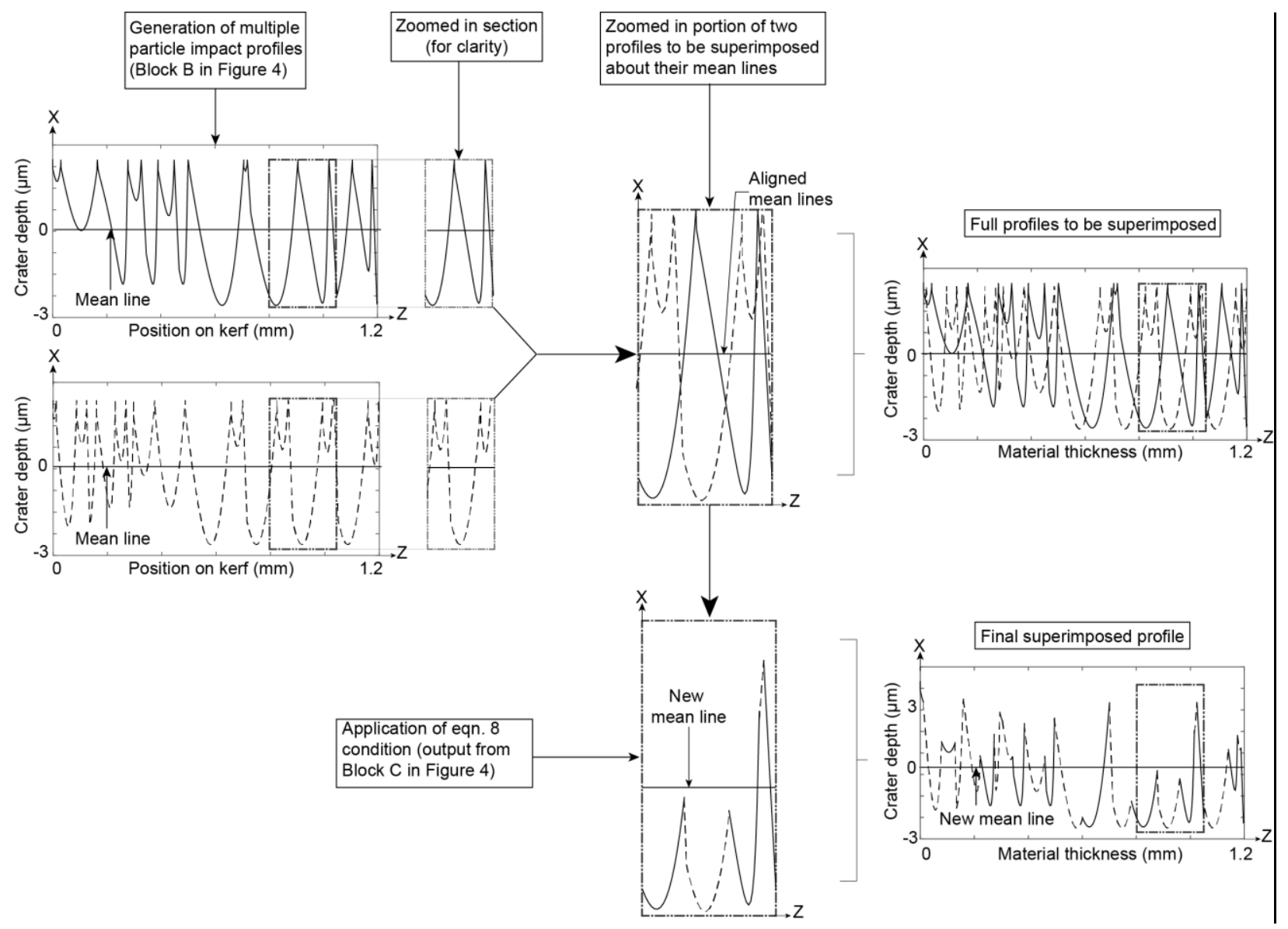

Fig. 2.12 Method to superimpose profiles. Solid horizontal lines represent means of the profiles.

The algorithm then marched along the $z$-axis comparing the $x$ coordinates of the current (superimposed) and new (from a set of single impacts) profiles, preserving the one that was deeper; i.e.

$$
\begin{aligned}
& x_{m, z}^{j}=x_{s, z}^{j} \quad \text { if } \quad x_{s, z}^{j}<x_{m, z}^{j-1} \\
& x_{m, z}^{j}=x_{m, z}^{j-1}
\end{aligned}
$$

where $j$ is the iteration number, and $x_{m, z}$ and $x_{s, z}$ are the depths of point $z$ for the multi-particle impact profile (block C in Fig. 2.6) and single particle impact profile (block B in Fig. 2.6), respectively. After the profiles were superimposed in this manner, the model generated a new unique single particle impact profile (Fig. 2.10), which was then again superimposed on the previous multi-impact profile. The model iterated in this manner until it reached a steady state surface roughness; i.e. where the difference in surface roughness between consecutive iterations 
was less than $0.001 \mu \mathrm{m}$. As can be seen in Fig. 2.11, the predicted roughness using this method was much closer to the measured value, and reached a steady-state profile after about 10 interations.

\subsubsection{D Model}

A three-dimensional (3D) roughness model was developed as an extension of the 2D model, except that the target now was a plane, and the craters were assumed to be conical. This allowed for craters to be randomly placed on a plane, as opposed to being concatenated on a line. The individual conical craters (Block D in Fig. 2.13) had a depth and width equal to the maximum depth and width predicted by the 2D single impact model (Section 2.3.3). The conical craters were superimposed on the plane at random impact locations (Block E in Fig. 2.13). The volume of the conical crater that penetrated into the virtual substrate created a permanent deformation of the substrate mesh (analogous to preserving the deepest point as was done for the 2D model), resulting in a roughened surface topology (Block F in Fig. 2.13). The dimensions of the virtual plane was $335 \mu \mathrm{m}$ x $1200 \mu \mathrm{m}$ (Block E in Fig. 2.13) and had a mesh resolution of $1 \mu \mathrm{m}$. The model continued to superimpose 3D craters until it reached a steady-state solution after approximately 50,000 iterations. The surface roughness of the 3D model was assessed using the approach of Jafar et al. [57], where 2D profiles were extracted along randomly placed lines on the $3 \mathrm{D}$ surface to yield 2D roughness profiles (Block $\mathrm{G}$ in Fig. 2.13). The $R_{a}$ value for a given condition was reported as the average of 5 such randomly extracted profiles. 


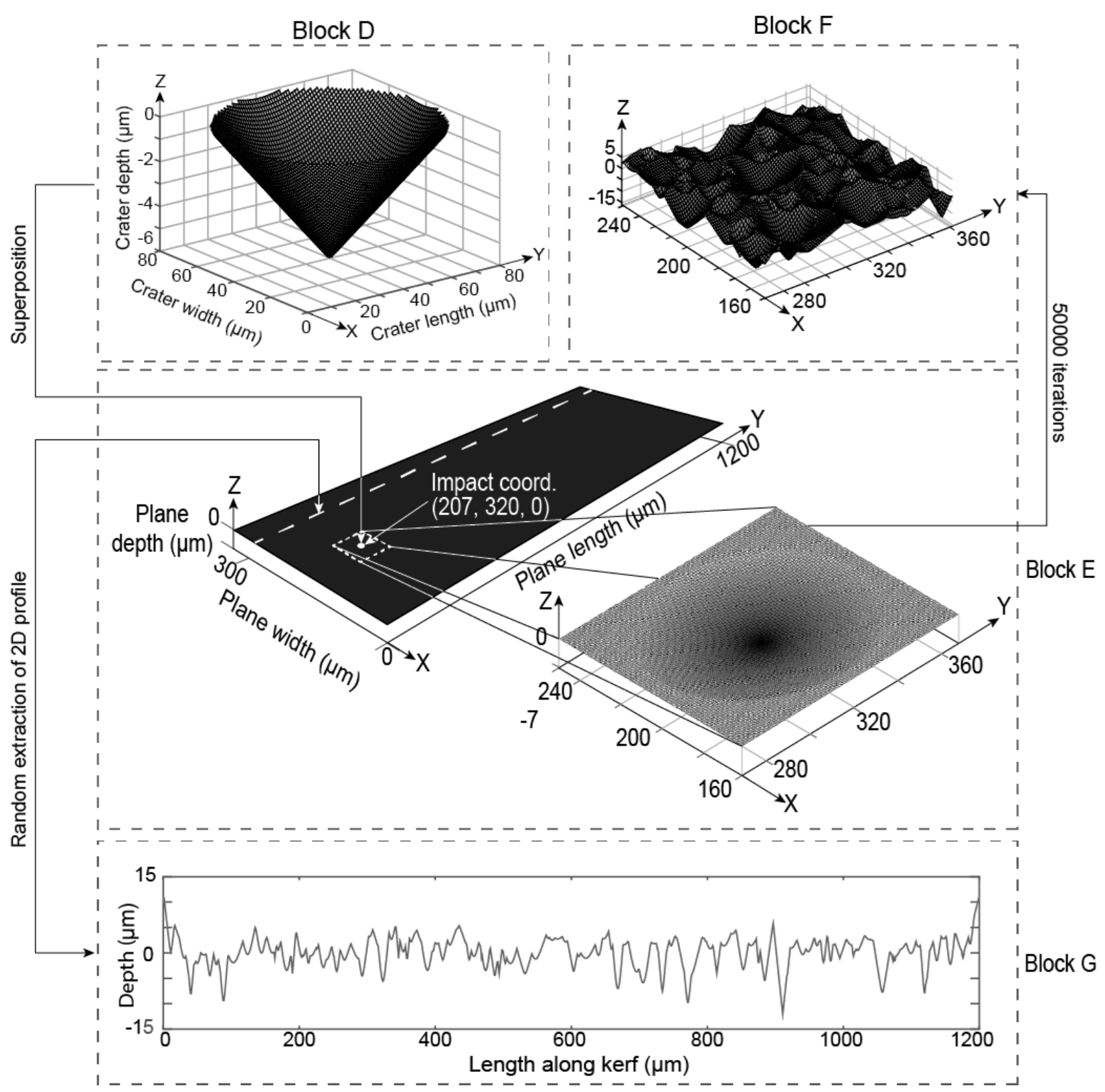

Fig. 2.13 3D surface model topology generation process. Block D shows an example of a generated 3D conical crater. Block E shows the superposition of the 3D conical crater on a segment of virtual kerf plane. Block F shows the surface topography of the virtual segment after 50000 iterations. Block $\mathrm{G}$ is the $2 \mathrm{D}$ profile extracted from a randomly generated line along the kerf. 


\subsection{Results and Discussion}

Fig. 2.14 shows the kerf of both carbon-fiber composite substrates after trimming using the $\mu \mathrm{AWJM}$. There was a relatively uniform surface texture, without striations and with minimal fiber pull-out, indicating a steady state cutting regime. The lack of delamination/fiber pullout was attributed to the use of the $\mu \mathrm{AWJ}$ nozzle, which greatly reduced the bending deformation of the work piece due to the small jet size.

a.
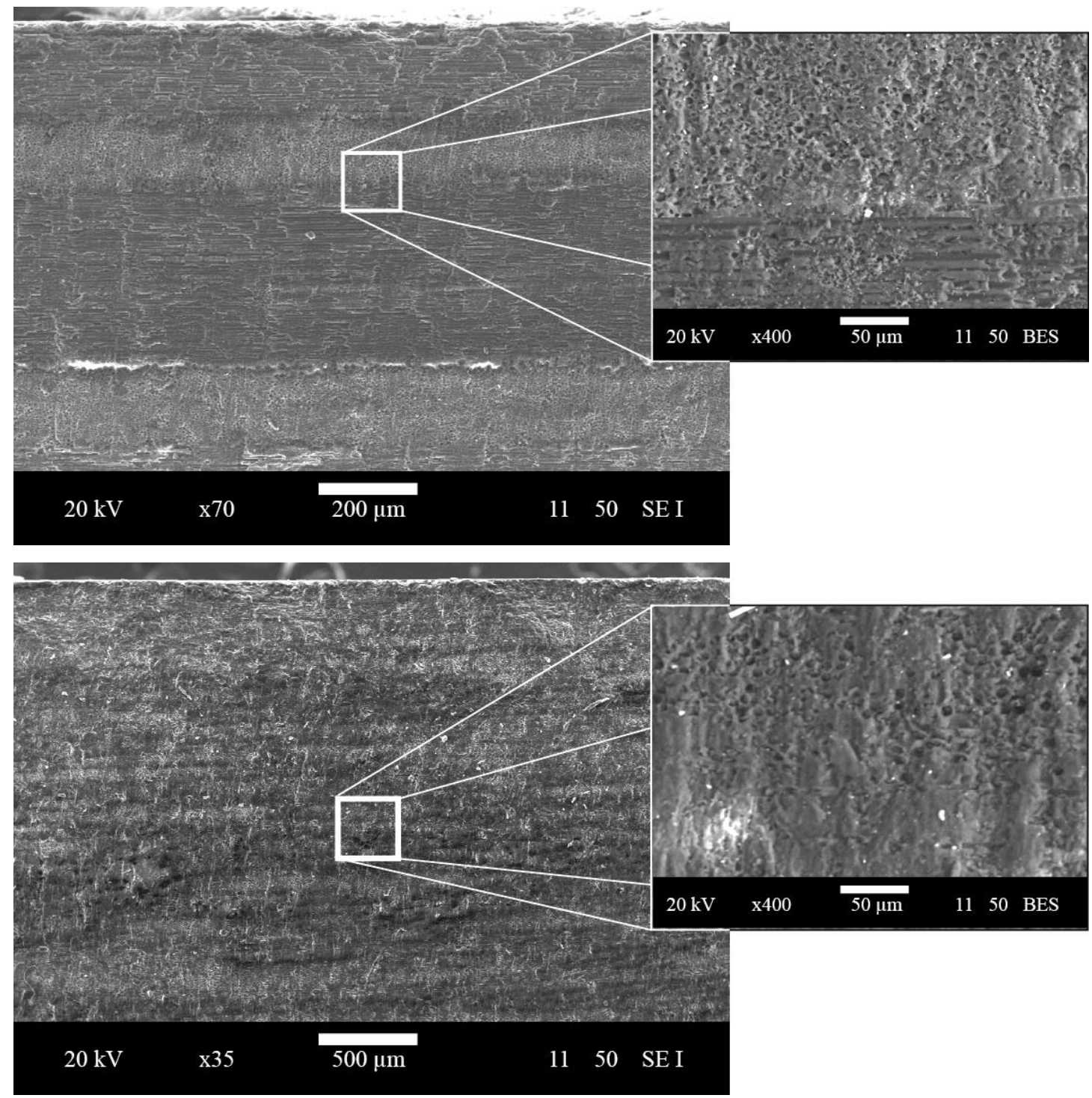

Fig. 2.14 Kerfs of composites trimmed with a $\mu \mathrm{AWJ}$ : a. CF-epoxy (ID 1 from Table 2.2) b. CFSMC (ID 16 from Table 2.2). Jet traversed from left to right. 


\subsubsection{Measured Dynamic Hardness}

Table 2.4 shows that the dynamic hardness of CF-epoxy was slightly higher than that of CFSMC. Dynamic hardness is often approximated as three times the tensile yield strength for homogeneous, isotropic materials [89]. The orthotropic properties of the CF-epoxy and CFSMC, caused by fiber reinforcement, made this approximation invalid, as seen by comparison with the tensile yield strengths of epoxy and vinyl ester resins; $80 \mathrm{MPa}$ [90] and $82 \mathrm{MPa}$ [91], respectively. This is consistent with the nano-indentation data of Qian et al. [92] on epoxy composites with carbon nano-tube silica fibers, and with the dynamic micro-indentation experiments on dental resin composites of Hirayama et al. [93].

Table 2.4 Dynamic hardness measurements on the target materials. Average particle and crater properties and standard deviations based on 20 measurements.

\begin{tabular}{llllll} 
Material & $\begin{array}{l}\text { Radius of } \\
\text { crater } \\
(\boldsymbol{\mu m})\end{array}$ & $\begin{array}{l}\text { Radius of } \\
\text { particle } \\
(\boldsymbol{\mu m})\end{array}$ & $\begin{array}{l}\text { Volume of } \\
\text { crater }\left(\boldsymbol{\mu m}^{\mathbf{3}}\right)\end{array}$ & $\begin{array}{l}\text { Particle } \\
\text { velocity } \\
(\mathbf{m} / \mathbf{s})\end{array}$ & $\begin{array}{l}\text { Dynamic } \\
\text { hardness } \\
(\mathbf{M P a})\end{array}$ \\
\hline $\begin{array}{l}\mathrm{CF}- \\
\text { epoxy }\end{array}$ & $38.2 \pm 5.9$ & $38.2 \pm 6.3$ & 79700 & 436 & 2450 \\
$\mathrm{CF}-\mathrm{SMC}$ & $38.9 \pm 7.5$ & $38.2 \pm 6.3$ & 94300 & 436 & 2070 \\
\hline
\end{tabular}

\subsubsection{Comparison of Predicted and Measured Roughness for 2D model}

The $2 \mathrm{D}$ roughness model required the superposition of approximately 15-25 roughness profiles to reach a steady-state value of $R_{a}$. and took 100-200 s to compute using an i7-4790k CPU, operating at $4.70 \mathrm{GHz}$, with $32 \mathrm{~GB}$ of RAM. As an example, Fig. 2.15 compares the measured and predicted steady-state surface roughness profiles on the CF-SMC substrate under the conditions of ID 10 in Table 2.2. 


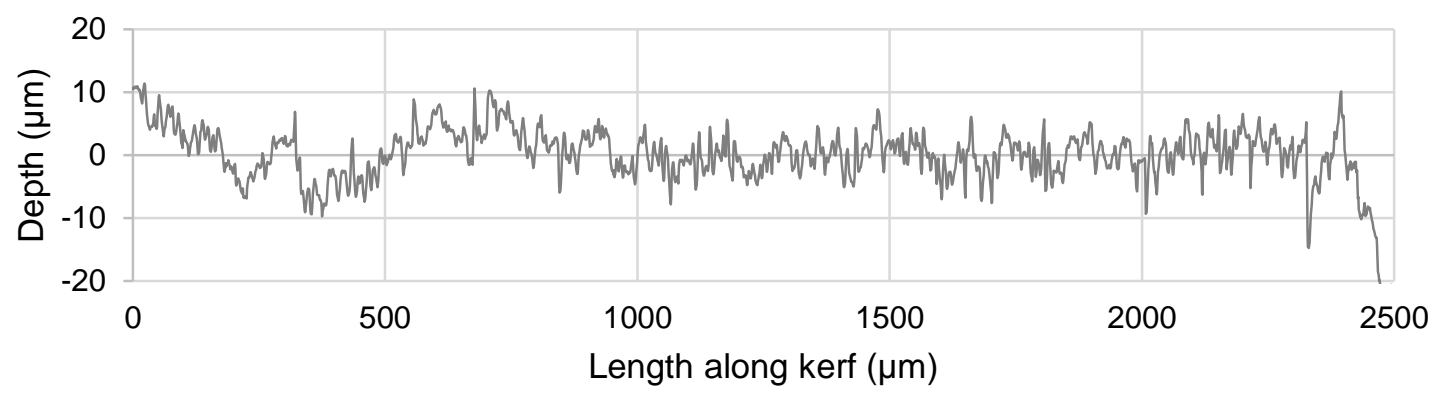

a.

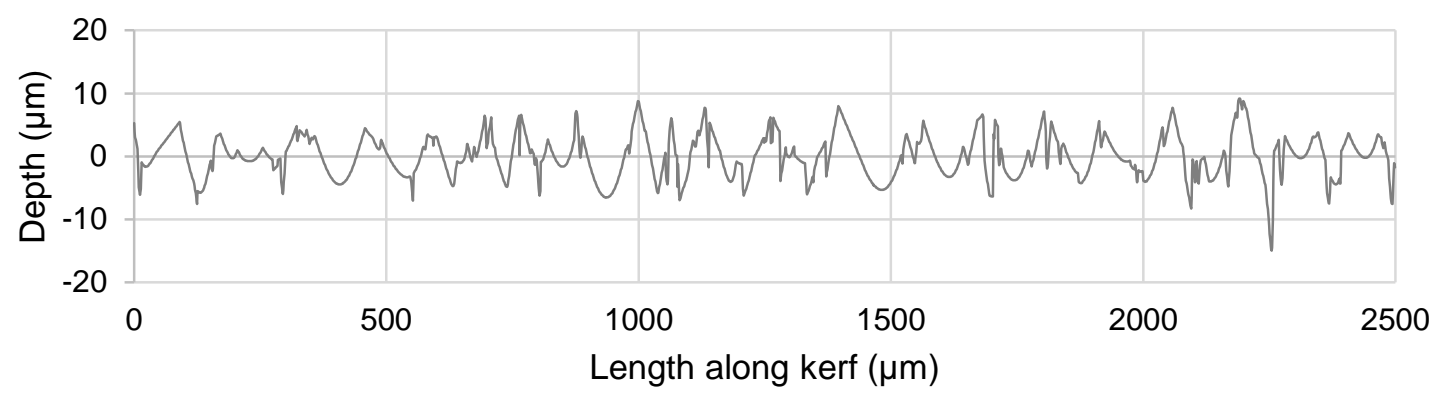

b.

Fig. 2.15 Surface roughness profiles for CF-SMC ID 10 in Table 2.2: a. measured $\left(\mathrm{R}_{\mathrm{a}}=2.41\right.$ $\mu \mathrm{m})$, and $\mathrm{b}$. predicted $\left(\mathrm{R}_{\mathrm{a}}=2.76 \mu \mathrm{m}\right)$.

The measured profiles (for both CF-epoxy and CF-SMC) exhibited a higher frequency of profile feature fluctuation than did the model profile, which tended to have larger peaks and troughs. This difference in profile morphology can be explained by the kerf surface microstructure and model simplifications. The craters generated by the rigid-plastic model (Fig. 2.9) were modelled by curve fitting a set of points that defined the shape of a single particle impact crater $[18,19]$. As a result, the superimposed multiple impact profiles were made up of a series of relatively smooth curves which neglected the microtopography created by fiber pullout and protruding fibers [29]. Additionally, the idealized rhomboids neglected the small-scale roughness features of the actual particles, which would have generated additional scratches not resolvable in the current model. The high resolution of the profilometer revealed these smallscale, high-frequency features of the machined kerf. Nevertheless, a Fourier analysis (FFT) of five representative predicted profiles showed that the dominant frequencies of the model remained constant regardless of the number of iterations, thus confirming that a steady state solution was achieved. Fig. 2.16 compares the FFT of the experimental (Fig. 2.15a) and 
predicted (Fig. 2.15b) surface roughness profiles for ID 10 in Table 2.2. Although the wavelength of the predicted model for the spectral component of maximum amplitude was slightly higher than that measured, the predicted and measured amplitudes were similar $(\sim 3 \mu \mathrm{m})$. Moreover, the amplitudes and trends of the spectra were similar at the dominant wavelength $<20$ $\mu \mathrm{m}$, with negligible amplitudes below $10 \mu \mathrm{m}$. As seen in the inset graphs of Fig. 2.16, the FFT for the model roughness profile showed a sharper decrease in the amplitude spectrum after the maximum confirming the previous observation that the experimentally measured profile contained higher frequencies resulting from small-scale features. As expected, the FFT of the predicted profile (Fig. 2.16b) did not exhibit significant high wavelength amplitudes since the model generated craters on a flat line, and thus produced negligible waviness.

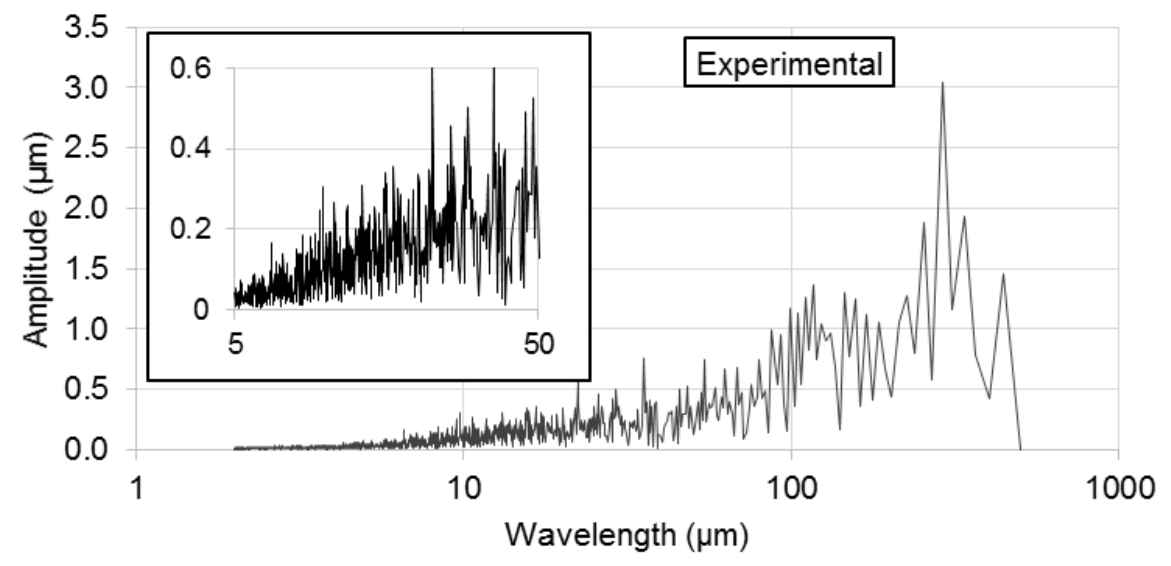

a.

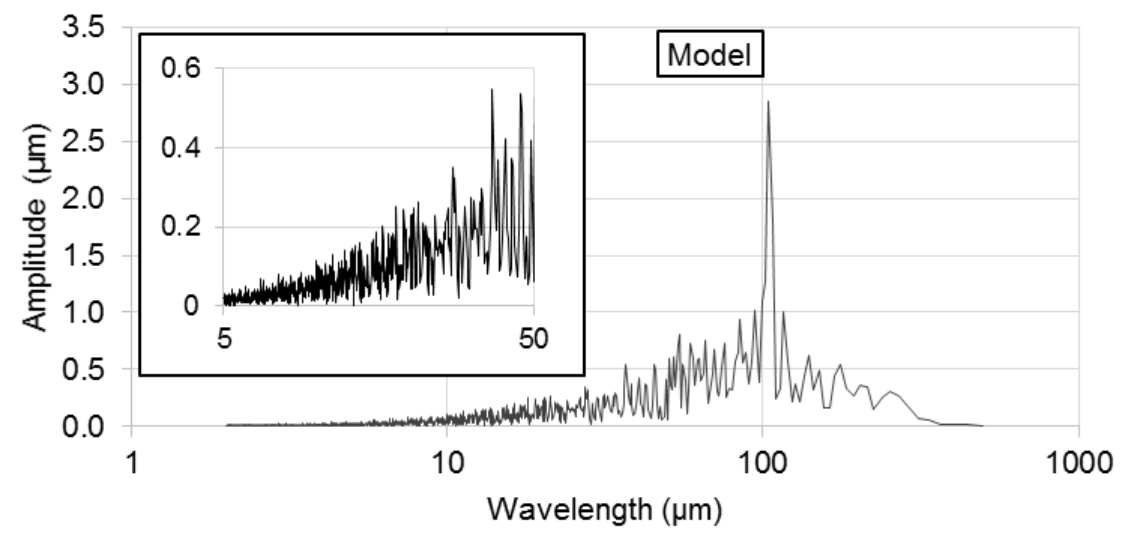

b.

Fig. 2.16 Fourier transform of: a. experimental, and b. model surface roughness profiles corresponding to ID 10 in Table 2.2. The inset shows magnified component of Fourier transform for smaller wavelength. 
Fig. 2.17 compares the predicted and measured maximum amplitudes extracted from the FFT analyses of all samples of Table 2.2 and the frequencies at which they occurred. The agreement between the maximum amplitudes in the model and measured spectra (Fig. 2.17) is reasonably good with an average difference of $14 \%$. Figure 2.17 shows that the predicted and measured maximum amplitudes for ID 10 were significantly higher than the other specimens. This was simply because that particular condition led to a relatively low erosive potential (i.e. lowest abrasive velocity, highest traverse speed, and lowest dose), which, when coupled with the higher thickness of the CF-SMC, resulted in a kerf with a very high taper, and thus high roughness.

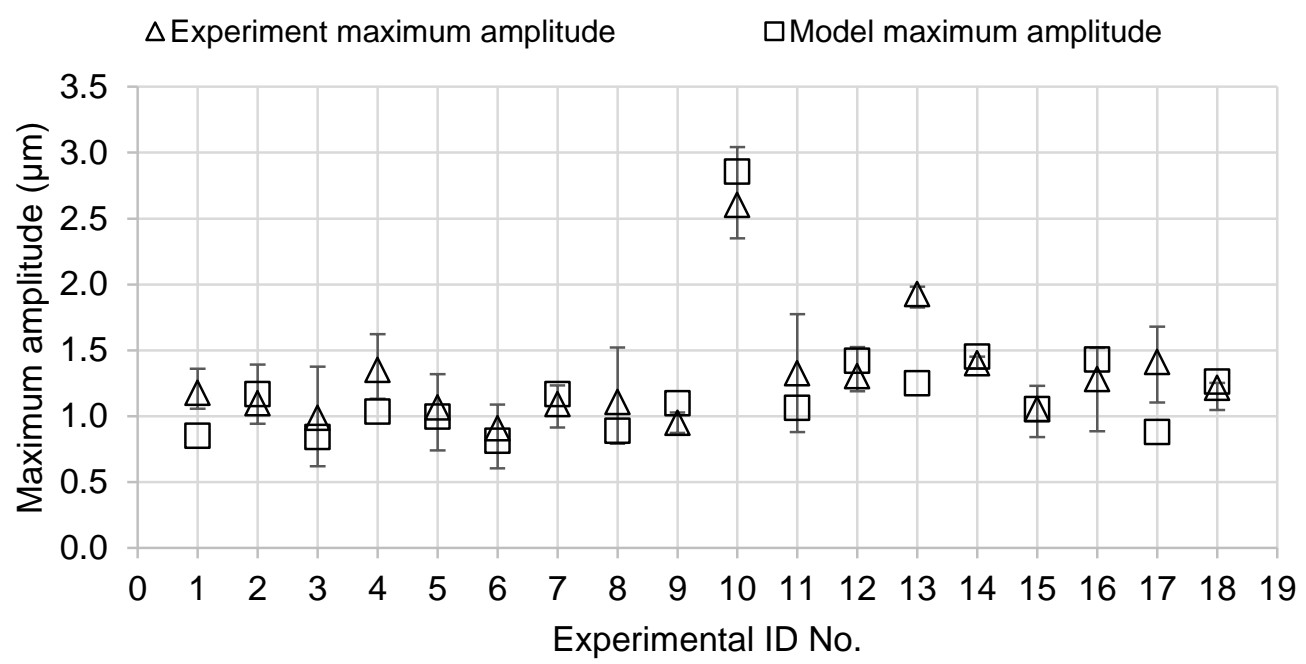

Fig. 2.17 Measured and predicted: a. maximum amplitude for all specimens shown in Table 2.2. Error bars correspond to maximum and minimum values of three measurements for each experimental specimen.

Profile skewness $\left(R_{s k}\right)$ is a measure of asymmetry in the distribution of the heights of the surface profile points, while kurtosis $\left(R_{k u}\right)$ can be used to compare the distribution of heights of the profile points to a normal (Gaussian) distribution for which $R_{k u}=3$ [94]. Fig. 2.18a shows that the skewness values of the experimental results (Table 2.2) were close to zero, consistent with the work of Kovacevic et al. [95] and Kunaporn et al. [96] that found little asymmetry in the shapes of peaks and valleys in an AWJ surface profile; i.e. the sharpness of peaks and valleys 
was similar. The model $R_{s k}$ results were relatively close to the measured values, although they were usually slightly negative, indicating a model profile that had sharper valleys and more rounded peaks. This is consistent with the profiles of Fig. 2.15 and was a consequence of protruding fibers/lips and the relatively smooth curves used to generate the model profiles, as discussed in Section 2.4.2.

Fig. 2.18b shows that the kurtosis values of the of the experimental and model results were generally quite similar and were less than 3 , indicating that in both cases the distribution of profile points was broader than would be expected of a Gaussian distribution. The slightly smaller values of $R_{k u}$ for the measured profiles are consistent with the presence of a greater fraction of small-scale roughness features as mentioned above in the context of the $R_{s k}$.

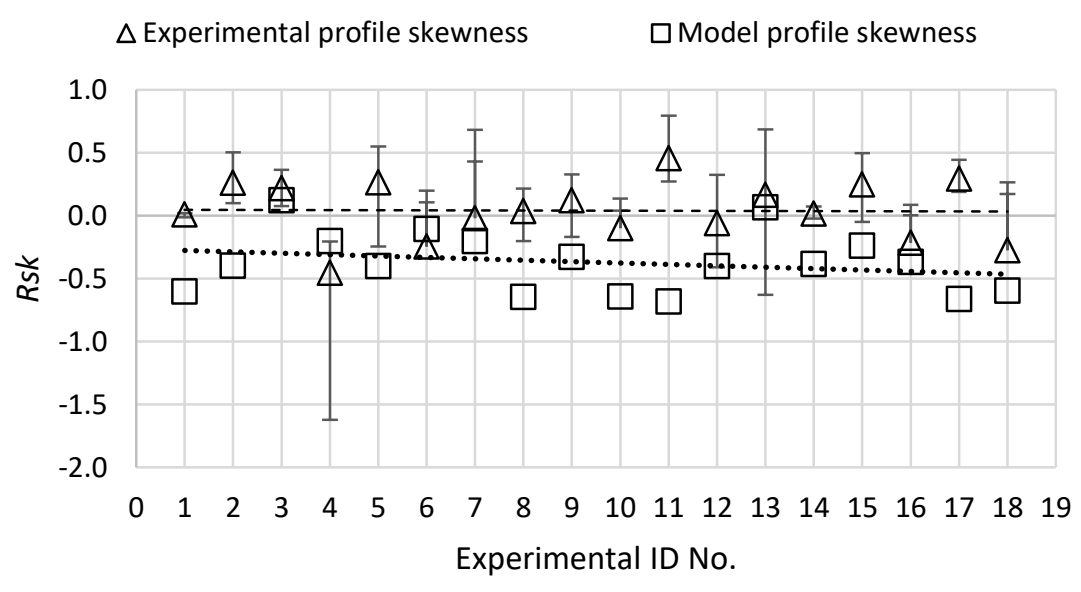

a.

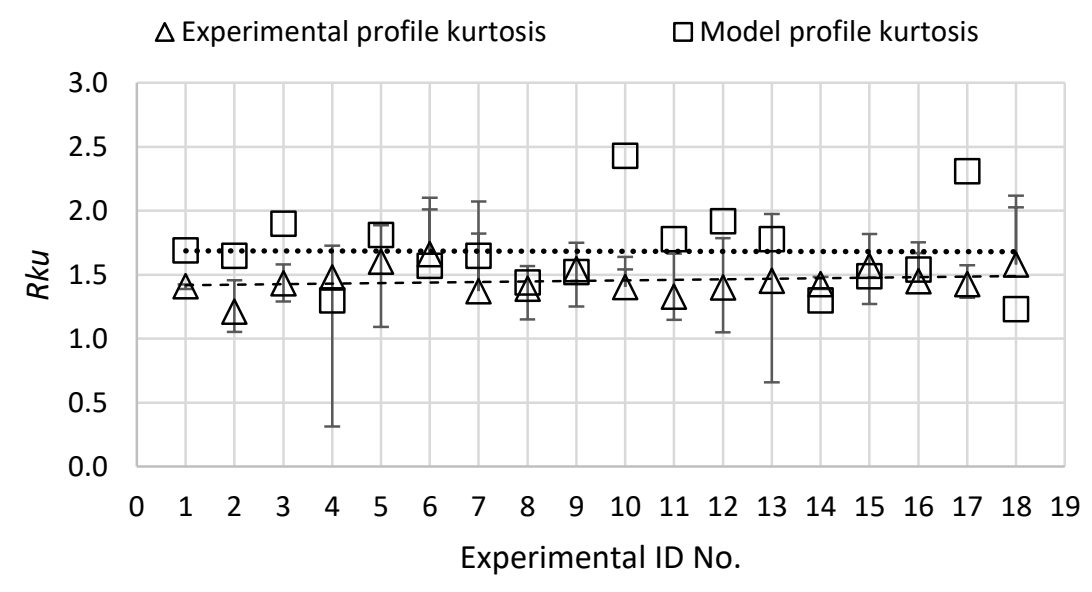


Fig. 2.18 a. Skewness, and b. kurtosis of the experimental and model profiles in Table 2.2. Error bars represent the difference between the minimum and maximum values of three measurements for each experimental sample.

Additionally, the rigid-plastic model did not account for the possibility of crater lip formation commonly associated with the oblique impact of ductile materials. However, these effects were minimized with the current materials, due to the high concentration of fibers in the relatively ductile matrix, resulting in effectively harder materials that produced smaller lips. This will be further discussed in Section 2.4.3.

Fig. 2.19 compares the predicted and measured $R_{a}$ values for the two target materials. The measured values offered a repeatability of $8 \%$ between tests on different days. The $2 \mathrm{D}$ surface roughness model agreed well with the experimental data, with a maximum error of $20 \%$ and an average error of $10 \%$.

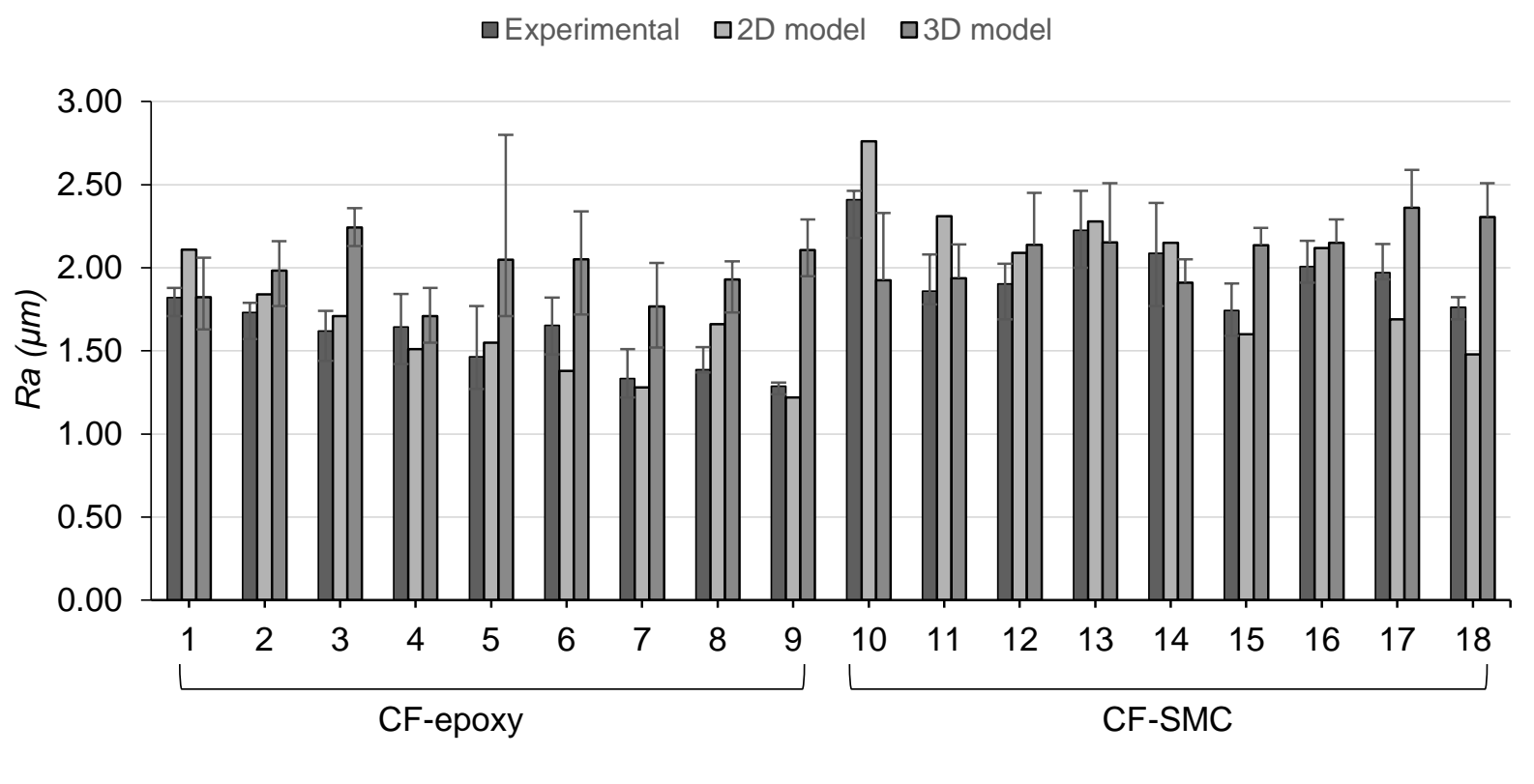

Experimental ID No.

Fig. 2.19 Experimental data, 2D model and 3D model roughness results for the experiments of Table 2.2. The error bars for the experimental results and the 3D model results correspond to the maximum and minimum surface roughness measurements on the same specimen (based on three and five measurements per specimen for the experimental and 3D model results, respectively). 


\subsubsection{Effect of AWJ Parameters on Roughness}

Based on the measured values in Table 2.2, Fig. 2.20 shows that the taper increased approximately linearly with increasing traverse speed, and hence decreasing dose on the kerf area. This is consistent with the observations of Shanmugam and Masood [84] when cutting layered composites, Azmir et al. [85] when AWJM aramid fiber reinforced plastics, Shanmugam et al. [86] when AWJM alumina ceramics and Zhoor and Nourain [60] when AWJM steel.

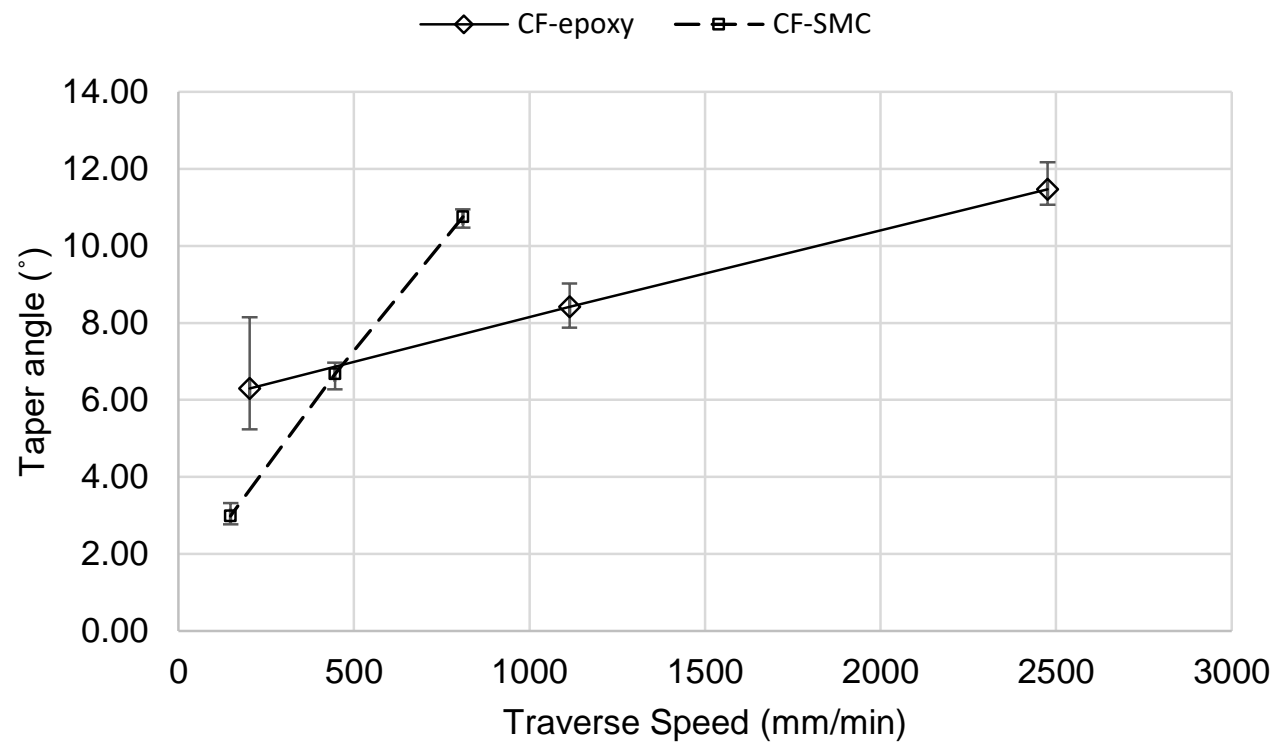

Fig. 2.20 Measured taper angle $(\beta)$ as a function of the nozzle traverse speed for the two materials $\left(P=211.5 \mathrm{MPa}, \mathrm{SOD}=1 \mathrm{~mm}, \dot{m}_{p}=0.5 \mathrm{~g} / \mathrm{s}\right)$.

Fig. 2.21 shows the relation between $R_{a}$ and taper for CF-epoxy and CF-SMC, with an average difference between the experimental and 2D model results of $9 \%$ and $11 \%$, respectively. The results show that increases in the taper angle as small as $5^{\circ}$ led to percentage increases in roughness as high as $27 \%$. The model confirms this trend, with higher taper angles resulting in a higher component of velocity perpendicular to the surface, leading to deeper impact craters that tended to increase the roughness. Since taper angle and traverse speed are directly related (Fig. 2.20), this implies that surface roughness increases with increasing traverse speed, and is consistent with the work of Azmir and Ahsan [37], Azmir et al. [85], Ramulu and Arola [62], 
Unde et al. [61], Wang [2] when AWJ cutting polymer composites, and many other investigators when AWJ cutting metals and metal composites.

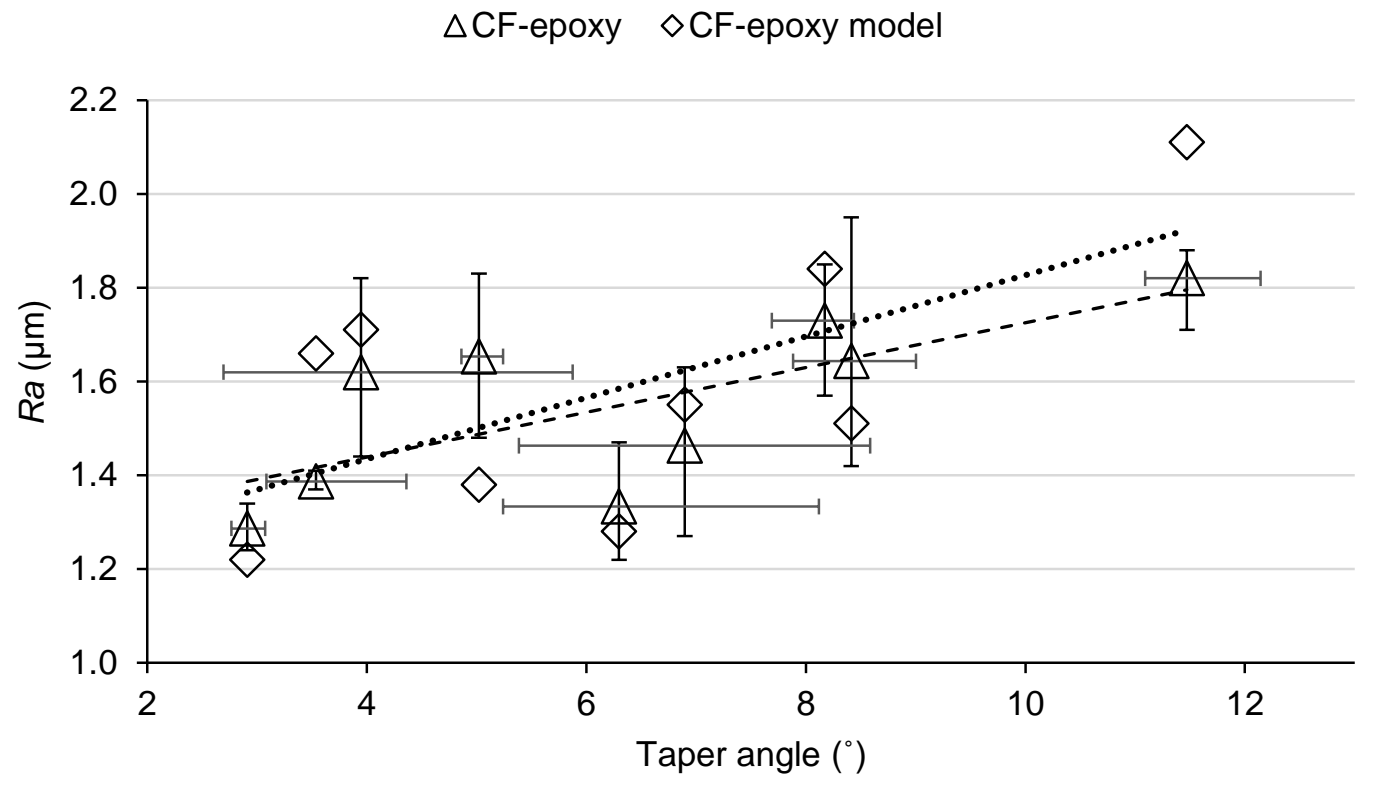

a.

OCF-SMC $\diamond C F-S M C$ Model

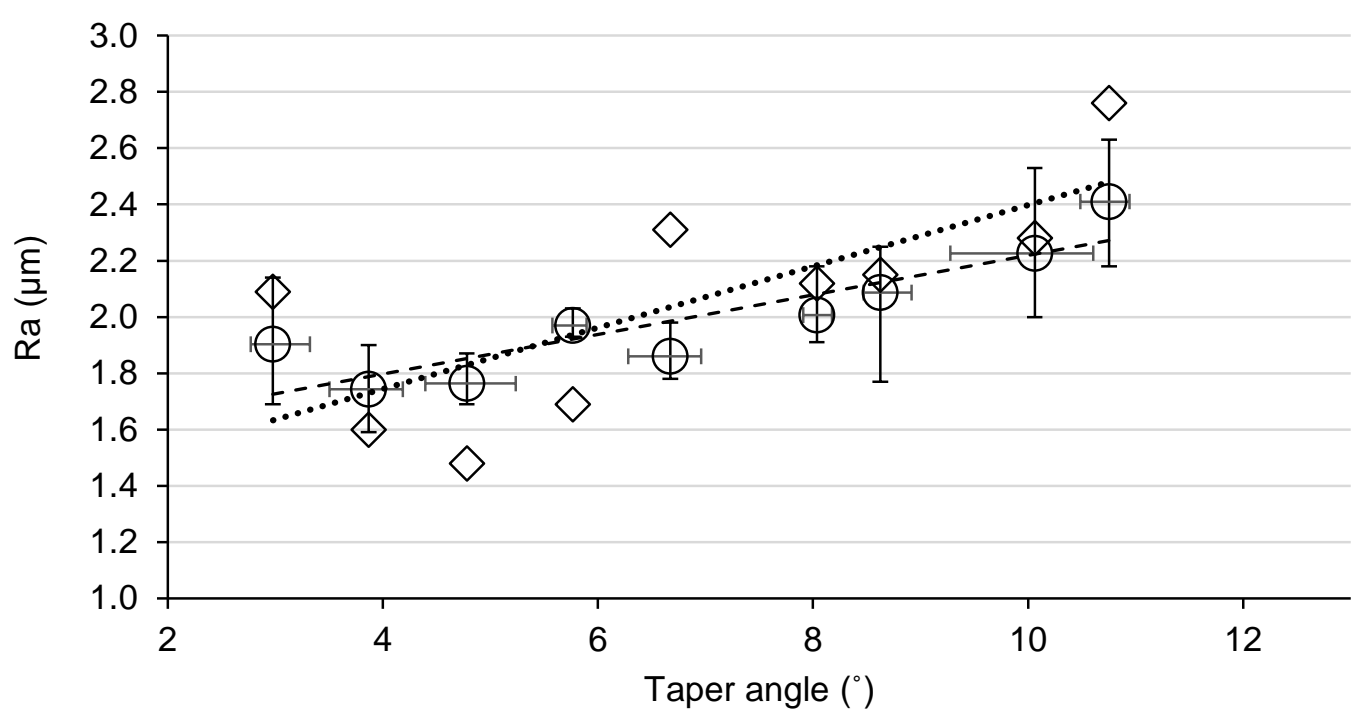

Fig. 2.21 $R_{a}$ vs. taper angle for a. CF-epoxy and b. CF-SMC, corresponding to the experimental conditions in Table 2.2. The horizontal and vertical error bars represent the maximum and minimum within the specimen measurements for the taper angle, and surface roughness, respectively. The dashed lines are the linear trend lines for the experimental and model data. 
Fig. 2.22 shows that roughness decreased with increasing particle velocity, which was primarily controlled by the pump pressure. This is consistent with the observations of previous researchers such as Azmir and Ahsan [37] for glass woven composites, Wang [2] for fiberreinforced polymer laminates [2], Azmir et al. [85] for aramid fiber reinforced plastics, Unde et al. [61] for carbon fiber reinforced plastics, and Ramulu and Arola [62] for graphite/epoxy laminates. This trend of decreased surface roughness with increased particle velocity appears to contradict the hypothesis used to explain the results of Fig. 2.21; i.e. that higher normal particle velocities result in increased crater depth, and thus increased surface roughness. However, this apparent contraction is resolved once it is realized that there is an interdependent relationship between particle velocity and taper, with higher velocities also leading to a reduced taper, which, as shown in Fig. 2.21, decreases the roughness. Based on the experimental data in Table 2.2 and Table 2.4, the taper can be approximated using an empirical model as a function of traverse speed $(U)$, particle velocity $\left(V_{p}\right)$ and dynamic hardness $\left(p_{d}\right)$ as

$$
\beta=-5.17+39.54 \cdot U+0.00168 \cdot V_{p}+0.00348 \cdot p_{d}-0.01976 \cdot U \cdot V_{p}-0.01092 \cdot U \cdot p_{d}
$$

with $R^{2}=0.89$ between experimental and empirical model results, and a correlation of $R^{2}=0.95$ and $R^{2}=0.90$ for CF-epoxy and CF-SMC, respectively, when considering $V_{p}$ and $U$. Since increasing the abrasive particle velocity in the model for a given constant taper was found to increase the surface roughness due to the generation of larger craters, it is likely that the reduced roughness in Fig. 2.22 mostly resulted from the decreased taper brought on by the higher velocity and specific doses, rather than the impact velocity itself. Equation (2.9) shows that increases in dynamic hardness will result in a decreased taper angle. However, this trend is bounded by a threshold traverse speed $(U), U_{\text {threshold }}=0.1825 \mathrm{~cm} / \mathrm{s}$ (i.e. $0.1825=0.00348 / 0.01092$ ), which is an impractically small traverse speed for the given materials. 


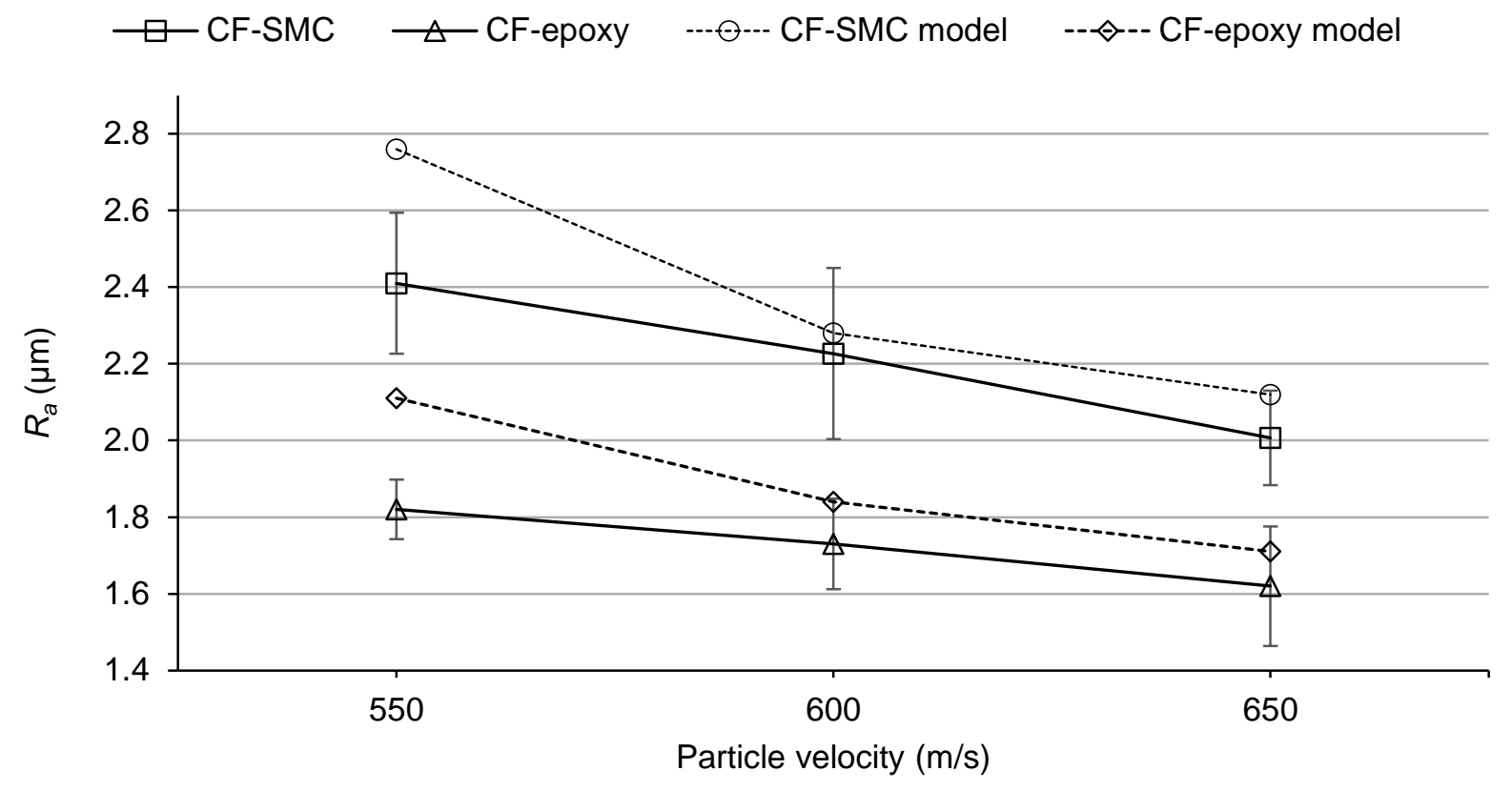

Fig. 2.22 Predicted (2D model) and measured $\mathrm{R}_{\mathrm{a}}$ as a function of predicted average particle velocity. Experimental values correspond to ID numbers: 1, 4, 7, 10, 13 and 16 in Table 2.2. Error bars show the standard deviation within each value based on three measurements.

The interdependent relationship between abrasive velocity, specific dose (equation (2.2)) and taper angle is shown by the surface plots in Fig. 2.23. Although there are some fluctuations due to inconsistent abrasive flowrate and pump pressure, the general trends show an increase in kerf taper (and hence impact angle) with decreases in dose and abrasive velocity. 
a.

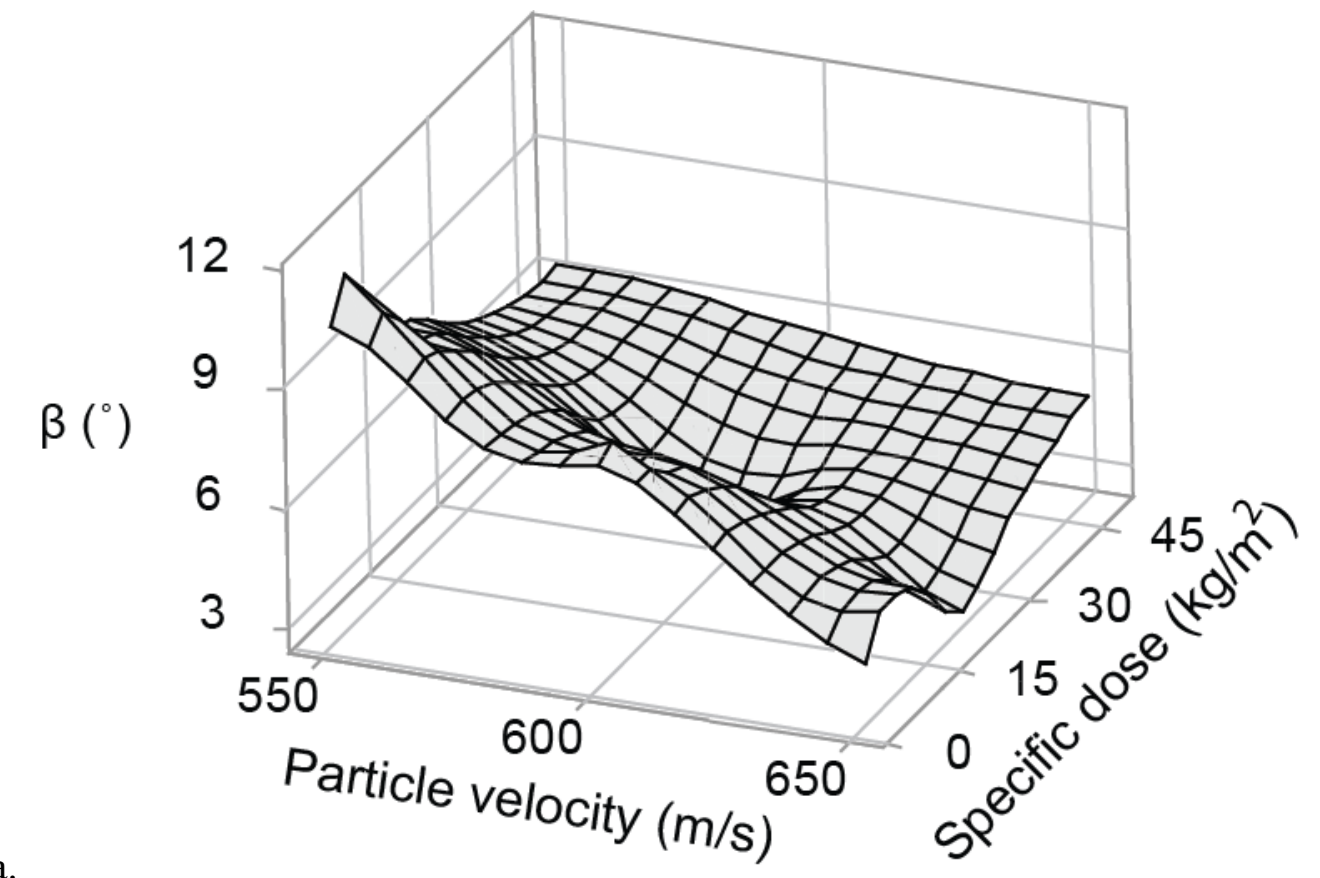

b.

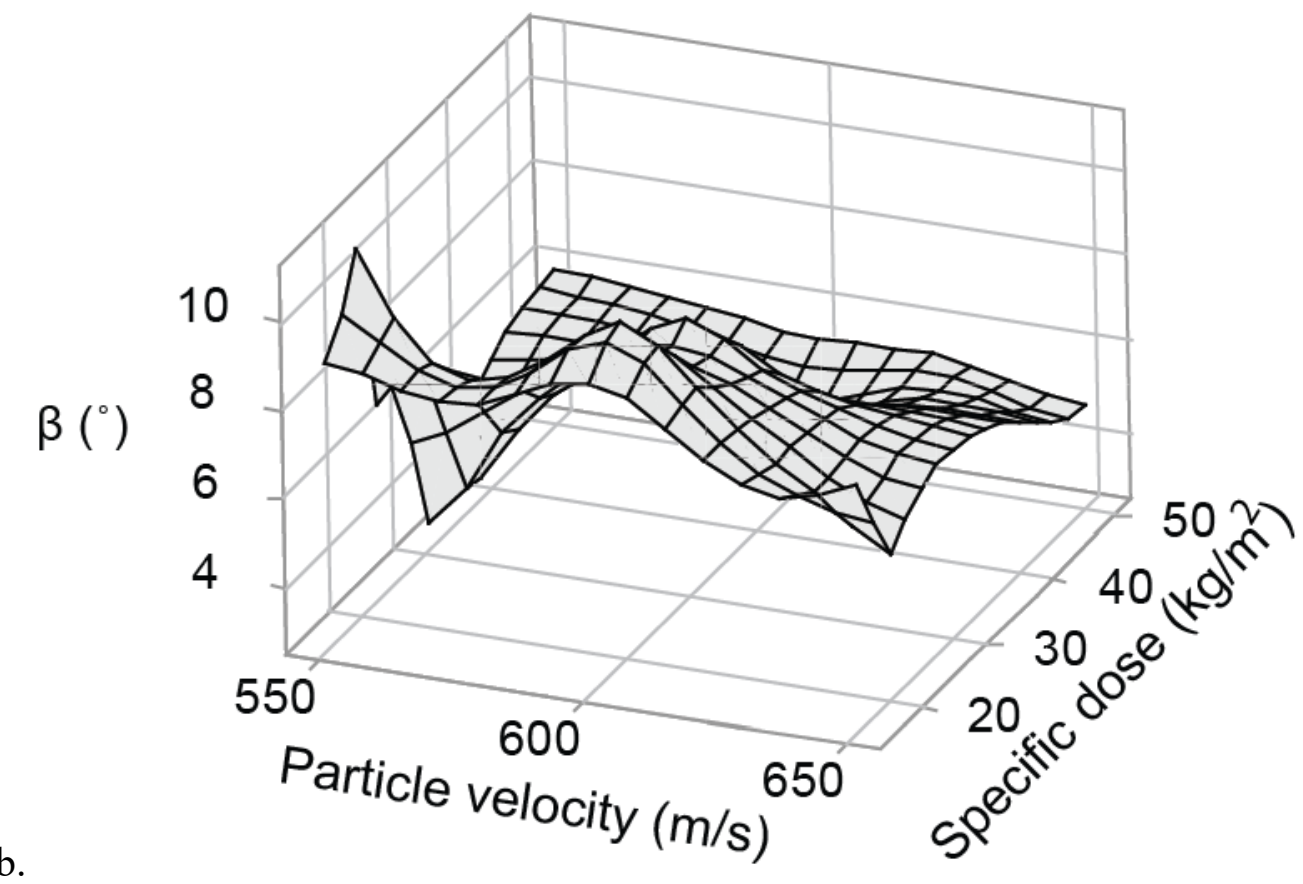

Fig. 2.23 Contour plots relating kerf projected dose, particle velocity and taper angle for a. CFepoxy, and b. CF-SMC.

Fig. 2.24 shows that surface roughness decreased with increasing material dynamic hardness. This is expected since a larger dynamic hardness resists indentation more, resulting in 
smaller craters. Although not directly presented, the same trend can be inferred by analysis of the roughness data presented by Akkurt et al., for AWJM cuts at a traverse speed of $20 \mathrm{~mm} / \mathrm{min}$ in pure aluminum, Al 6061, Brass 353, AISI 1030 and 304 SS [97], assuming that the dynamic hardness and yield strength are directly related. Zeng [67] developed a semi-empirical model for determining a material's AWJ machinability value; i.e. its propensity to erode. If Zeng's [67] machinability index is calculated for the materials in Akkurt et al. [97], the results indicate that the surface roughness decreases with decreasing machinability index. This supports the trend of increasing surface roughness with decreasing dynamic hardness (Fig. 2.24), the latter being inversely proportional to the extent of particle erosion. This implies that dynamic hardness can also be used to assess a material's AWJ machinability.

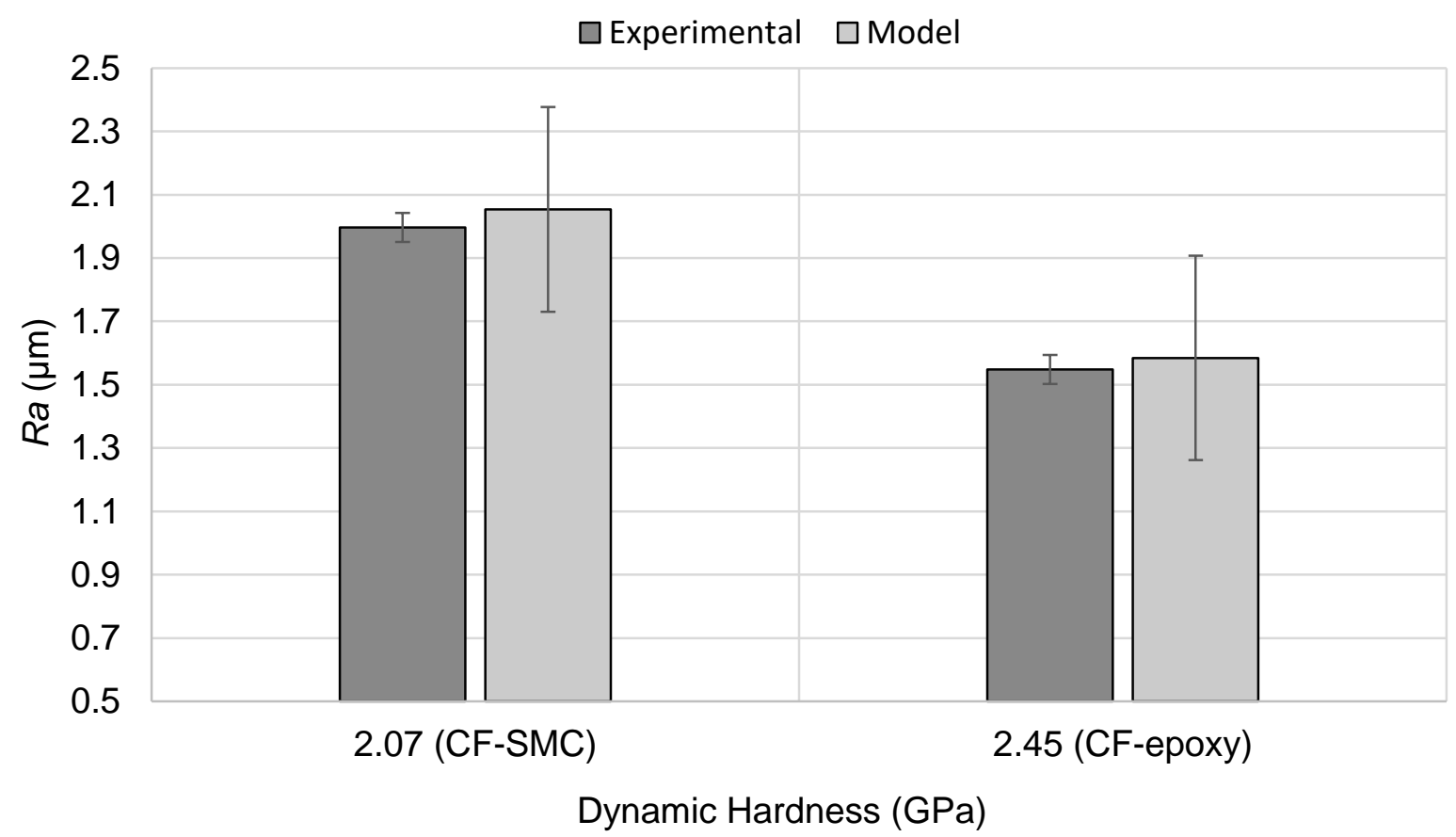

Fig. 2.24 $R_{a}$ as a function of target dynamic hardness (based on the average $\mathrm{R}_{\mathrm{a}}$ for all test cases for CF-epoxy and CF-SMC, respectively). Predicted results are from 2D roughness model. 


\subsubsection{Sensitivity Analysis}

A sensitivity analysis was conducted using the $2 \mathrm{D}$ surface roughness model in order to determine the relative influence of the model inputs (block A, Fig. 2.6). In other words, each parameter in Table 2.5 was individually varied, keeping the remaining variables constant at the baseline level 2.

Table 2.5 Model input parameters for sensitivity analysis.

\begin{tabular}{llll}
\hline Parameter & Level 1 & Level 2 & Level 3 \\
\hline Particle velocity $(\mathrm{m} / \mathrm{s})$ & 200 & 400 & 800 \\
Particle size $(\mu \mathrm{m})$ & 50 & 100 & 200 \\
Angularity $\left(^{\circ}\right)$ & 22.5 & 45 & 77.5 \\
Particle density $\left(\mathrm{kg} / \mathrm{m}^{3}\right)$ & 1000 & 2000 & 4000 \\
Impact angle $\left(^{\circ}\right)$ & 22.5 & 45 & 77.5 \\
Dynamic hardness $(\mathrm{MPa})$ & 1000 & 2000 & 4000 \\
\hline
\end{tabular}

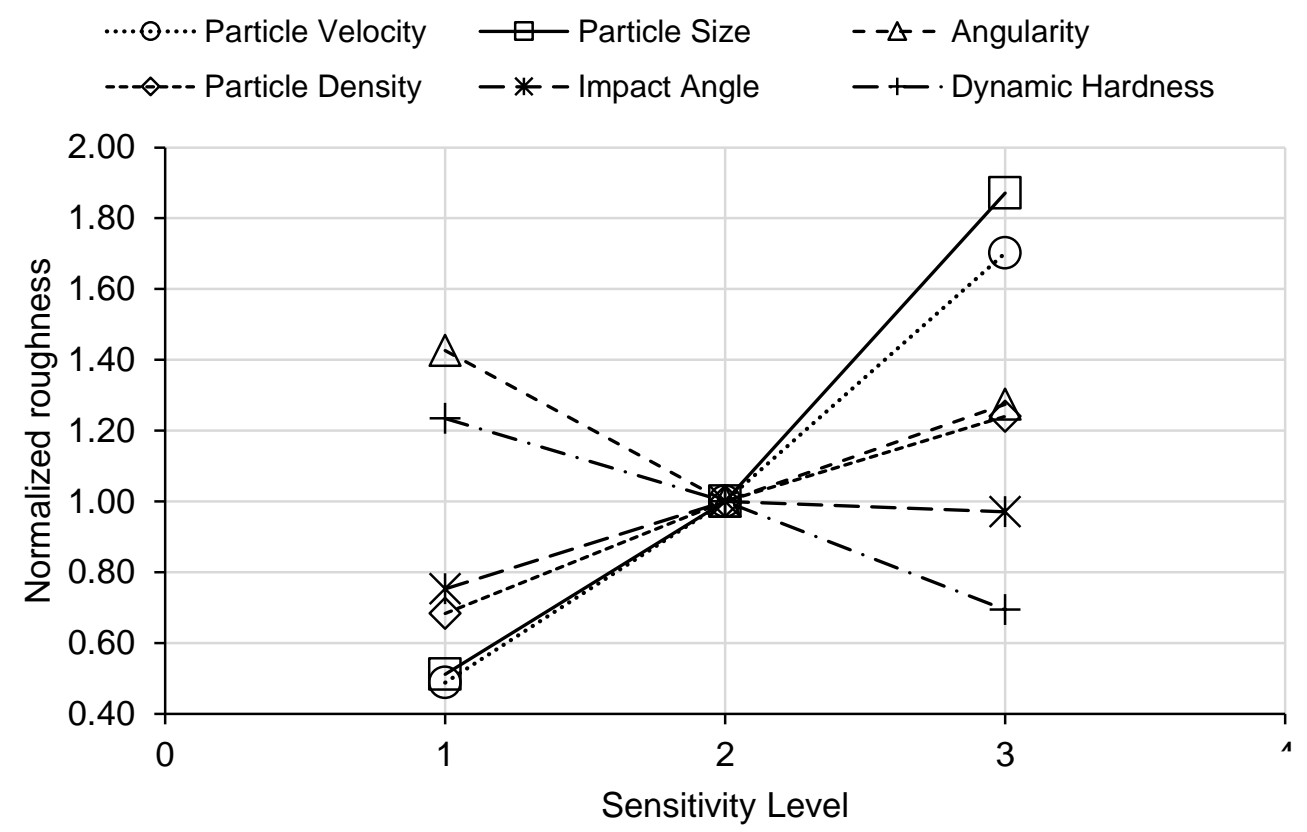

Fig. 2.25 Sensitivity analysis for the surface roughness input factors with input parameters corresponding to Table 2.5 . 
Fig. 2.25 shows that particle size and particle velocity had the most significant effect on surface roughness, followed by particle density, impact angle and angularity. The results of the sensitivity analysis show that particle velocity (viz. pressure) has a larger effect on surface roughness than impact angle (viz. taper), which has been unresolvable from experimental testing due to the interdependent relationship between pressure and traverse speed on AWJ cuts/trims. The result of the sensitivity analysis also shows that surface roughness is more dependent on the kinematics of the particle such as size and momentum, than the incident conditions. It was expected that particle angularity would have the least significant effect considering the symmetry of the idealized rhomboid particle and that the orientation of the particle was randomized. Lastly, dynamic hardness is shown to have the third largest difference on surface roughness, after size and velocity.

\subsubsection{D Model Results}

Fig. 2.26 compares a profilometer surface scan of specimen ID 1 in Table 2.2 to its replicated surface using the 3D model, and one of the randomly extracted 2D profiles. A steady-state solution of the 3D model required approximately 50,000 iterations for a virtual plane size of 335 $\mu \mathrm{m} \times 1200 \mu \mathrm{m}$, and required approximately 8 hours to complete using the computer specifications described in Section 2.4.2. The surface roughness predictions of the 3D model had an average error of $16 \%$, which typically overestimated the experimental measurements when tested for all conditions shown in Table 2.2. 


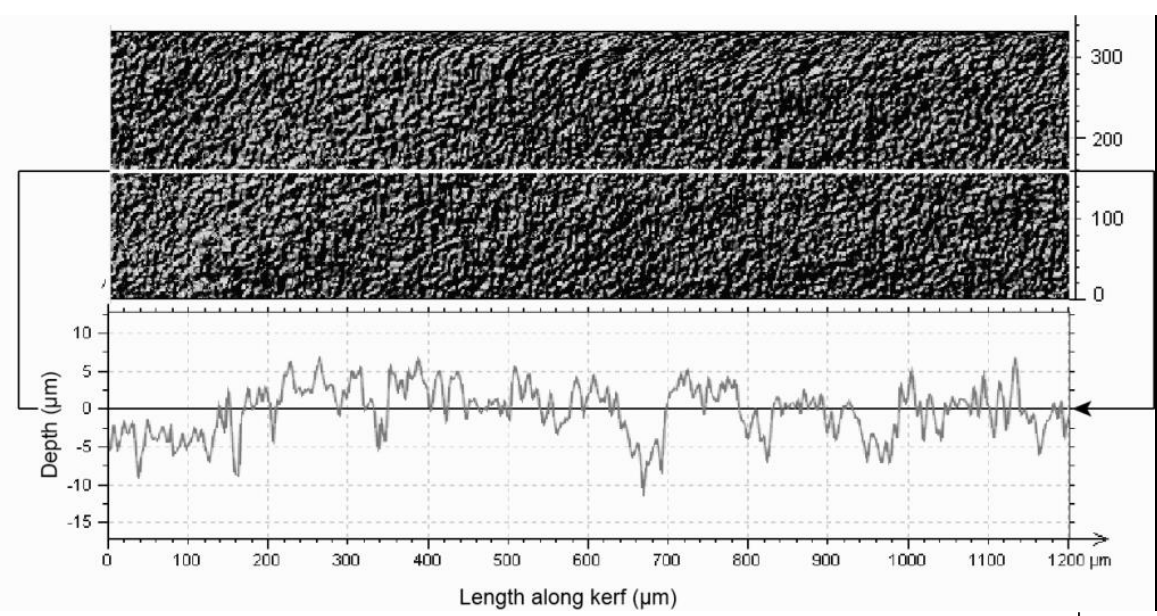

a.

b.

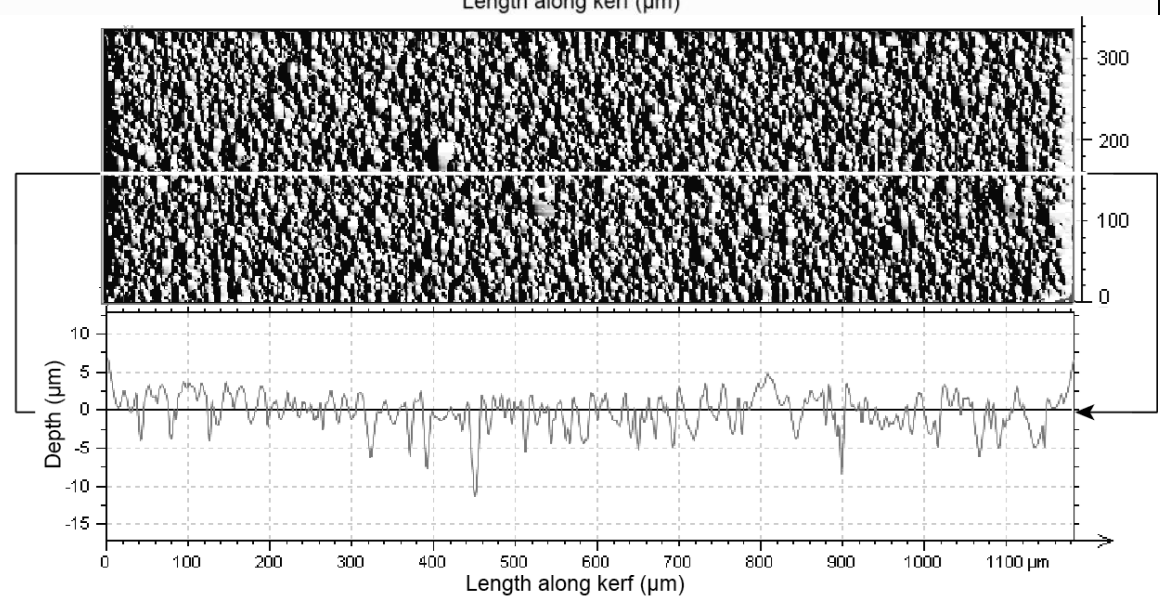

Fig. 2.26 3D surface topography and 2D extracted profiles for ID No. 1 in Table 2.2 for: a. experimental measurement, and b. 3D model results.

Qualitatively, the 3D surface roughness model and the experimental surfaces were similar, consisting of small indentations and peaks; however, idealized perfectly circular craters are visible in the $3 \mathrm{D}$ surface roughness model. When comparing the extracted $2 \mathrm{D}$ profiles of the $3 \mathrm{D}$ model surface (Fig. 2.26), experimental (Fig. 2.15a), and 2D model profiles (Fig. 2.15b), the 3D model more closely resembles that of the experimental profiles. This can be attributed to the differences in superposition, where the 3D model surface generation process more closely represents the stochastic impacts associated with the single particle erosion action occurring during AWJM, unlike the 2D model, which concatenated crater profiles along a line. Additionally, the low-frequency and wider craters observed in the experimental profile can be attributed to alternative mechanisms of material removal, such as fiber roll-out/pull-out, which were included in the model. Despite the advantages of the 3D model, i.e. the ability to randomly 
place craters on a plane, the $2 \mathrm{D}$ model roughness predictions were closer to the measured. The assumed conical craters apparently did not sufficiently capture the irregularities in crater shape that arose due to the varying particle trajectories as they ploughed and cut the substrate.

\subsubsection{Limitations}

The current model is limited in its ability to treat highly ductile/fibrous composite substrates that are prone to produce large lips during single particle erosion. For example, a third composite was modeled: polypropylene with $30 \%$ by wt. randomly oriented long glass fiber (PPLGF) (Fig. 2.28). Compared to CF-epoxy and CF-SMC, the PPLGF composite had a relatively high thermoplastic resin content, with fiber and matrix properties resulting in increased ductility and reduced hardness. As shown in Fig. 2.27, specimens for two typical test cases showed that the model consistently underestimated the measured roughness, because the rigid-plastic model used to predict the craters could not account for the extensive protruding lips and fibrous nature of the composite (Fig. 2.28). The effect of such protruding material was negligible in the CF-epoxy and CF-SMC samples.

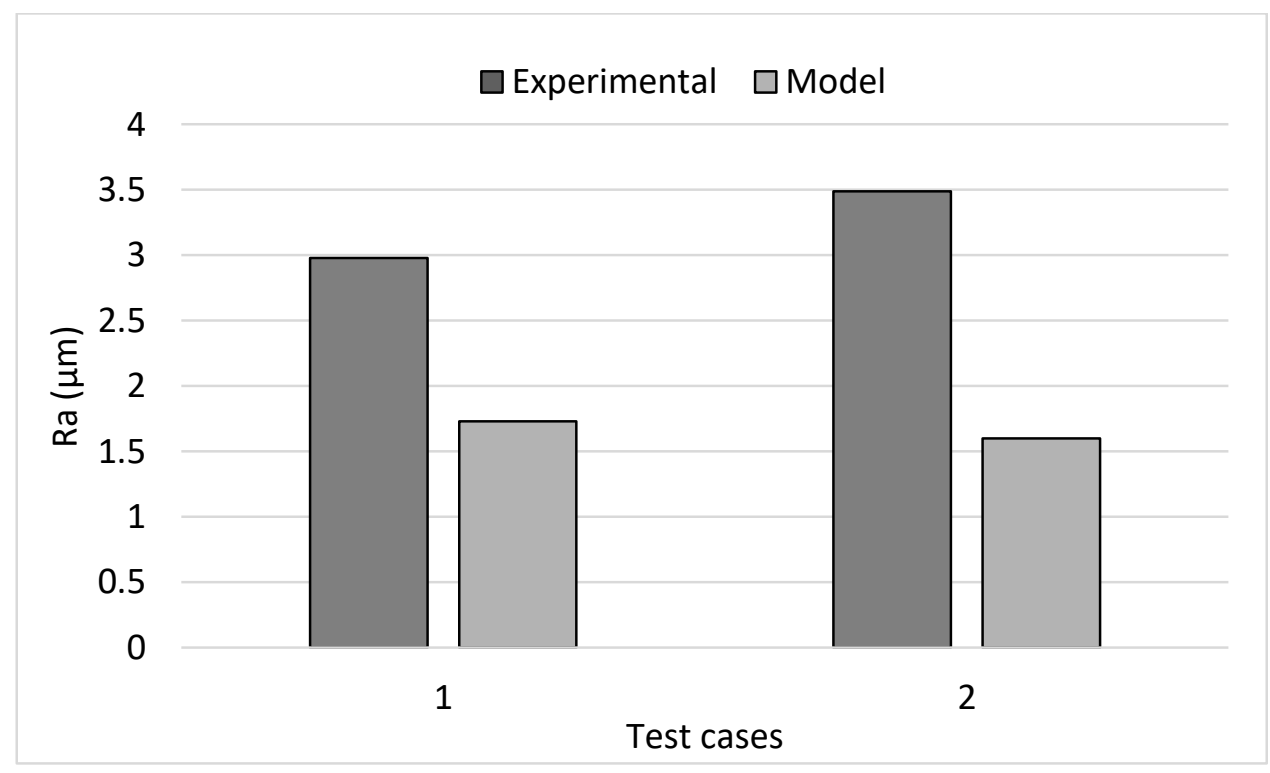


Fig. 2.27 Experimental vs. model results for two test cases of the PPLG composite machined using $75 \mu \mathrm{m}$ garnet at $\dot{m}_{a}=0.5$ with $A_{l}=32^{\circ} \pm 22^{\circ}, A_{2}=58^{\circ} \pm 22^{\circ}$. Test 1: $P=242 \mathrm{MPa}, V_{p}=589$ $\mathrm{m} / \mathrm{s}, U=0.0042 \mathrm{~m} / \mathrm{s}, \beta=2.08$. Test $2: P=195 \mathrm{MPa}, V_{p}=524 \mathrm{~m} / \mathrm{s}, U=0.0033 \mathrm{~m} / \mathrm{s}, \beta=1.83^{\circ}$.

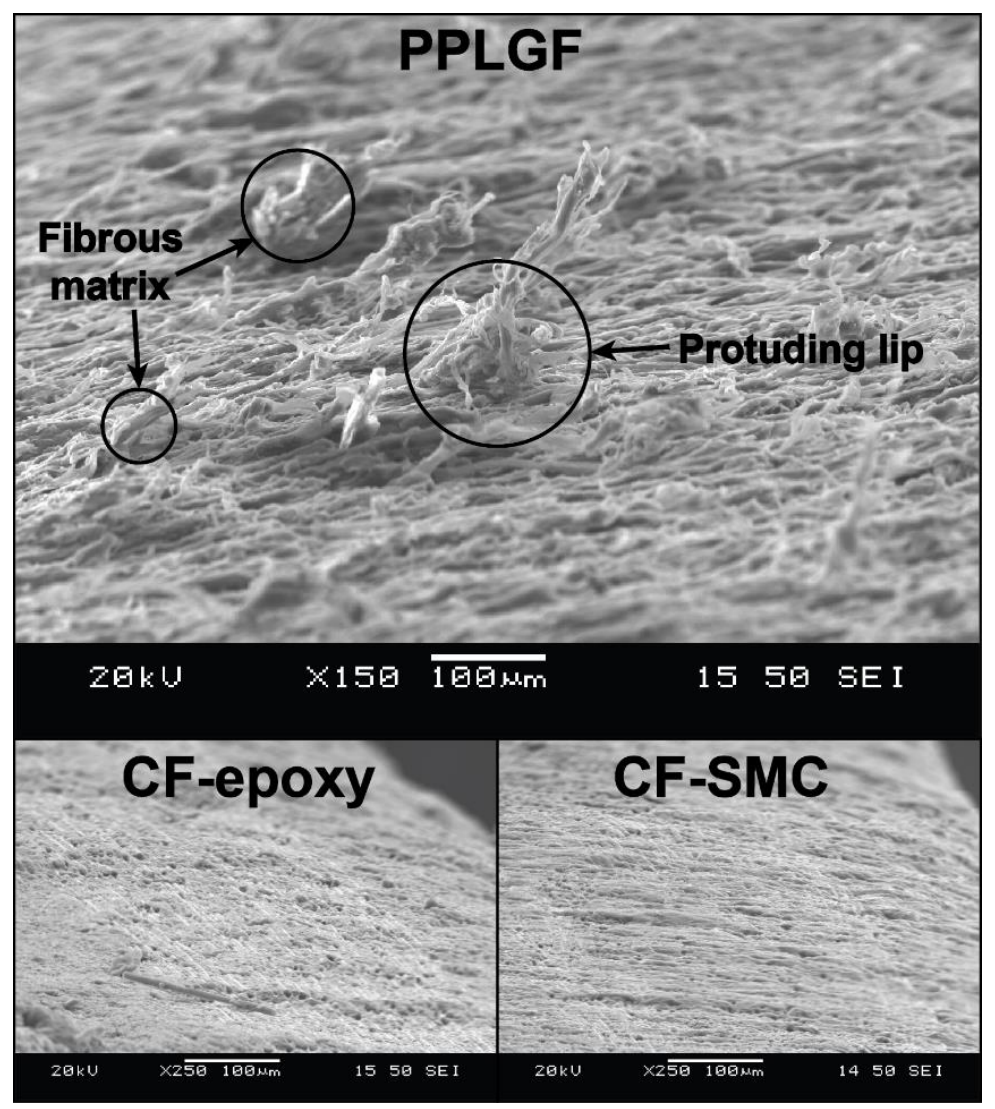

Fig. 2.28 Comparison of oblique scanning electron micrographs of the $\mu \mathrm{AWJ}$ cut surfaces for the three composite substrates.

A second limitation of the model is the required knowledge of the kerf taper in order to solve for the local particle impact angle. In the present case, this was obtained from an empirical relation based on experimental measurements under different process conditions. The model did also not take into consideration the damage of the water, which could affect the surface topology. 


\subsection{Summary}

Two and three-dimensional models were developed for predicting the tapered kerf surface roughness when trimming two types of composites. Both models superimposed crater profiles inferred from a rigid-plastic single impact model. The predicted surface roughness values were compared to measured profiles from trimming operations performed over a wide variety of operating conditions. The 2D and 3D models were found to predict the steady-state surface roughness with average errors of $10 \%$ and $16 \%$, respectively.

Increases in particle velocity were found to decrease the surface roughness because the associated increase in jet energy reduced the kerf taper, resulting in more oblique impacts that tended to erode the profile peaks. Similarly, increases in taper were associated with increases in surface roughness due to the increase of the local particle impact angle. Decreases in material dynamic hardness resulted in larger individual impact craters, and ultimately a higher roughness. An empirical taper model was developed to relate particle velocity, traverse speed and substrate dynamic hardness, and it was found that increases in particle velocity and particle specific dose resulted in an increased taper, whilst an increase in dynamic hardness resulted in a decreased taper. A sensitivity analysis revealed that surface roughness was affected by the particle size and velocity, more than the particle impact angle, and by the target dynamic hardness.

The model and experimental trends correlated well with the existing literature. Because the model is based on fundamental erosion principles, it is likely to find application for other classes of materials that operate in the ductile erosion regime, and for other AWJM operations. It is also possible that the model can be used for other manufacturing processes involving particle erosion such as air-based abrasive jet micromachining, and abrasive slurry jet micromachining.

Surface roughness is an important metric when quantifying AWJ cut quality in composite substrates, however delamination is also commonly generated during piercing and cutting AWJM processes. Chapter 3 will model the delamination processes that result from the hydraulic shock generated during liquid jet impact during the AWJ piercing process. 


\section{Chapter 3 Modelling of Delamination due to Hydraulic Shock when Piercing Anisotropic Carbon-Fiber Laminates using an Abrasive Waterjet}

This chapter is based on the following published paper:

J. Schwartzentruber, J.K. Spelt, M. Papini. "Modelling of delamination due to hydraulic shock when piercing anisotropic carbon-fiber laminates using an abrasive waterjet", International Journal of Machine Tools and Manufacture, vol. 132, pp. 81-95, 2018

\subsection{Introduction}

AWJ delamination damage in composite materials is a two-step process: i. damage initiation, and ii. crack propagation via hydrodynamic pressurization [44, 45]. Shanmugam et al. [45], Wang [2], and Hashish [98] have partially attributed the fracture initiation in composite materials

to hydraulic shock during AWJ piercing operations. Although previous studies have been mostly qualitative, with little work regarding the underlying mechanisms, it is generally agreed that crack initiation and propagation is most likely to occur at the resin-rich interface between laminate plies [29]. Ho-Cheng [44] has modelled delamination due to hydrodynamic pressurization during the AWJ drilling process using a LEFM approach, but models of initiation do not yet exist.

During AWJM, a high-velocity liquid jet forms a vacuum in the mixing chamber, thus entraining abrasive particles into the fluid jet. However, at startup the jet making the initial impact in most water-jet systems does not yet contain particles, and so it has little or no cutting action. The initial impact of this pure water jet in operations such as piercing generates high transient pressures $[99,100]$ that can lead to substrate damage [73, 101-105]. The most commonly used approximation of the impact hydraulic pressure, $p_{i}$ is [100]

$$
p_{i}=\rho c v
$$

where $\rho$ and $v$ are the fluid density and velocity, respectively, and $c$ is the speed -of -sound in the fluid. 
Hydraulic shock [99, 100, 106-109] and the hydrodynamics of a pure liquid jet impact [103, 110-117] on isotropic targets have been studied in detail, and found to result in a symmetric pressure distribution on the target. However, since admittance, i.e. deformation of the target due to the hydraulic shock loading, affects the magnitude of the impact pressure [107-109], the anisotropic response of the composite under shock loading is expected to result in an asymmetrical displacement field and thus pressure distribution. Very few studies exist of the interaction of high-pressure AWJs with anisotropic composite laminates. Zhang and Li [103] described AWJ impact based on previous literature, but they provided no experimental or modelling evidence for their conclusions.

Fig. 3.1 describes the AWJ impact process on a composite. Upon impact, the fluid is arrested almost instantaneously [117], and a release wave that propagates radially at the acoustic wave speed is formed [108]. At the point of initial contact, the periphery of the jet expands faster than the release wave [108], thus forming a shock front inside the jet. The water between the substrate and shock front is continually compressed $[100,107,108]$ as subsequent 'layers' of arriving fluid are decelerated through the front [99]. The compressed fluid begins to be released as the shock front detaches from the substrate as a contact angle $\beta$, which grows until a critical value $\beta_{c}$ is reached (Fig. 3.1), and the expanding shock front surpasses the jet contact periphery. At this point, lateral jetting [108] of the compressed liquid occurs, accompanied by a reduction in pressure [107-109]. Once abrasive particles are entrained into the jet, material removal begins, and the pressure reduces to its quasi-steady state stagnation value $[103,110]$.

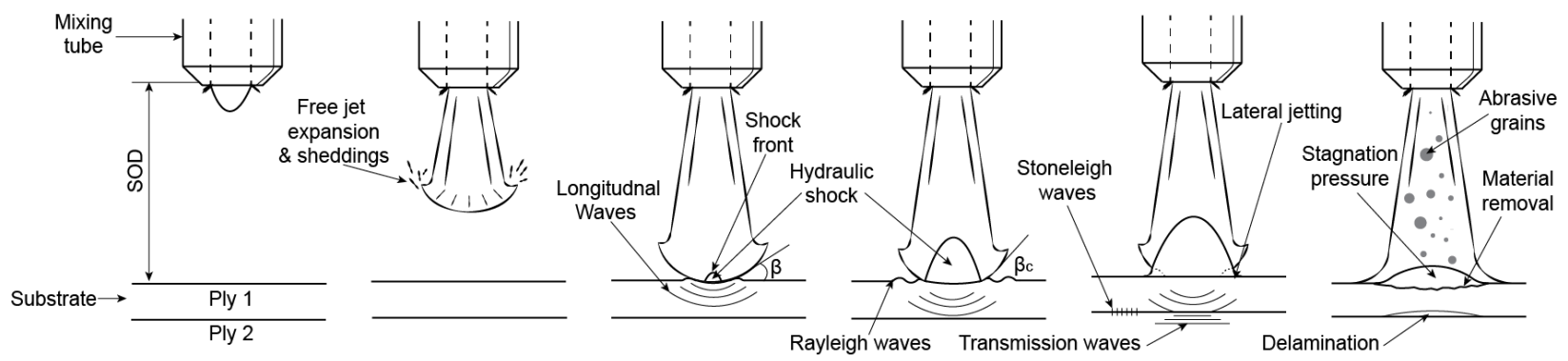

Fig. 3.1 Schematic impact process of AWJ piercing on laminates. 
The rapid energy transfer between the waterjet and laminate resulting from the hydraulic shock generates localized deformation and longitudinal, shear, Rayleigh, and Stoneleigh stress waves that travel at different velocities depending on their direction $[118,119]$ within the anisotropic laminate [120, 121]. For example, carbon-fiber epoxies have acoustic velocities of 10.8 and $3.04 \mathrm{~km} / \mathrm{s}$ in the longitudinal and transverse directions, respectively [122]. Consequently, the wave patterns in such anisotropic materials are very complex, comprising of multi-modal wave frequencies travelling in various directions at different velocities [120]. Upon impact, the elastic waves emanate with a compression front, followed by a tensile relief wave [120] which results in the initiation of damage [121]. Brunton [123] observed that the shape of impact damage in PMMA was velocity dependent and that the damage zone transitioned from ring fracture to an annular depression of plastic deformation at impact velocities greater than 450 $\mathrm{m} / \mathrm{s}$. As abrasive is subsequently entrained in the jet and material is removed, and the initiated cracks are exposed and subjected to hydrodynamic pressurization leading to crack propagation $[44,45]$.

The small spatial and temporal scales of jet impact have motivated the use of hydrocodes and numerical simulations to study the strains and stresses involved. All previous numerical work focused on the impact on isotropic materials. For example, Bourne performed two-dimensional simulations of water jets impacting poly-methyl methacrylate (PMMA) [114] and water [124] using the EDEN hydrocode. Hsu et al. [110] generated a three-dimensional fluid-structure interaction (FSI) model of a water jet impacting PMMA using ABAQUS. Both authors reported hydraulic impact pressures that were in good agreement with the Cook [100] one-dimensional impact model (Eq. 1) and determined the equivalent stresses induced in the material. However, the investigators employed explicit formulations which do not account for waterjet drag effects such as ablation as the jet travels through ambient air [115]. Furthermore, in the model of Hsu et al. [110], the liquid slug was modelled using a hemispherical front, but in reality the jet front is slightly flattened due to aerodynamic ablation $[112,125]$, following a $1 / 7$ th power law shape [126]. Since it has been shown that the coherence, shape, and size of the jet plays a significant role in the shock loading [127], such formulations may yield inaccurate results.

Out-of-plane interlaminar stresses $\sigma_{z}, \tau_{x z}$, and $\tau_{y z}$ (relative to the coordinate system in Fig. 3.2) are responsible for the interlaminar crack generation [128]. Delamination of interlaminar 
interfaces under impact is a complex phenomenon and has been attributed to several mechanisms such as the spatial distribution, amplitude and duration of stress waves, and the delamination strength of the interlayers [129]. Gorham and Field [121] found that large shear stresses along the resin-rich interfacial layer of composites arise from the normal incidence of the highintensity compressive pulse associated with liquid impact. Johnson [130] has shown that a compressive pulse develops hoop stresses parallel to the propagating wave front and tensile stresses normal to the wave tail, which act along the interface to promote ply separation [121]. Wilkinson and Reynolds [131] have shown that impact energy is radiated in the form of shear waves propagating along an interface which can promote debonding [121]. Interlaminar spall fractures have been observed in glass-fiber composites after liquid jet impact [121].

In summary, the majority of liquid jet impact studies have been highly theoretical/analytical with little work using higher order numerical modelling approaches. They focus on the highpressure phenomena within the liquid jet, rather than the material response to it. Of the studies that conducted numerical modelling, most are two-dimensional, and none consider prediction of the initiation of damage in anisotropic materials. For anisotropic materials, the dependence of contact angle on the anisotropic deflection of the substrate requires the development a threedimensional FSI model. The present research paper investigated the interaction of AWJs and subsequent initiation of delamination in a carbon-fiber/epoxy (CF-epoxy) symmetric laminate using a three-dimensional FSI model that implements cohesive zone modelling (CZM). The model was restricted to the prediction of damage initiation due to the initial jet impact on the uneroded target, and did not consider crack propagation due to hydrodynamic pressurization or the bending of the bottom plies as the jet approached the break-through of the laminate. The numerical results are compared to delamination measurements made on pierced specimens using micro-tomography.

\subsection{Experiments}

AWJ piercing experiments were performed on a CF-epoxy laminate with the aim of identifying the mechanisms of delamination as a function of operating pressure and nozzle size. 
Delamination was measured using 3D x-ray micro-tomography and compared with the FSI model predictions.

\subsubsection{Piercing experiments}

A continuous carbon fiber ( $60 \%$ by wt.)/epoxy, laminate made of 6 uni-directional plies in the stacking sequence shown in Fig. 3.2 was used in the experiments and having the properties given in Table 3.1. The mode II critical fracture energy $\left(G_{I I C}\right)$ was not provided by the manufacturer, and thus approximated based on fracture data of Fernlund et al. [132] for a similar toughened epoxy, where $G_{I C}=215 \mathrm{~J} / \mathrm{m}^{2}$ and $G_{I I c}=580 \mathrm{~J} / \mathrm{m}^{2}$. It was assumed that difference between $G_{I I C}$ of the composite provided by the manufacturer, and that of Fernlund et al. [132] was equal to the difference between their $G_{I C}$ values (i.e. $G_{I I C}=580-(215-137)=502 \mathrm{~J} / \mathrm{m}^{2}$ for the present epoxy). Also following [13], the fracture envelope (i.e. the criterion for mixed-mode fracture) was assumed to follow the relation $\frac{G_{I}}{G_{I C}}+\frac{G_{I I}}{G_{I I C}}=1$ for toughened epoxy resins [133, 134]. The $0^{\circ}$ fiber orientation angles were aligned with the $x$-axis Fig. 3.2.

Table 3.1 Composite material properties as provided by the manufacturer. Moduli and Poission's ratio correspond to properties of a single ply. Tensile strength and tensile modulus corresponded to properties of the composite stack.

\begin{tabular}{ll}
\hline \multicolumn{1}{c}{ Property } & \multicolumn{1}{c}{ Value } \\
\hline Matrix & Epoxy \\
Reinforcement & Continuous carbon fiber \\
Fiber weight fraction $\left(W_{f}\right)$ & 0.6 \\
Fiber orientation & Uni-directional \\
Total thickness $(t)(\mathrm{mm})$ & $1.2 \pm 0.017$ \\
Specific Gravity & $1.53($ ASTM D792 [135]) \\
Tensile Strength $(\mathrm{MPa})$ & $1277($ ASTM D3039 [136]) \\
Tensile Modulus $(\mathrm{GPa})$ & $122($ ASTM D3039 [136]) \\
$E_{11}(\mathrm{GPa})$ & 117 \\
$E_{22}=E_{33}(\mathrm{GPa})$ & 8 \\
$G_{12}=G_{13}(\mathrm{GPa})$ & 5.1 \\
$G_{23}(\mathrm{GPa})$ & 3.74 \\
$v_{12}=v_{13}$ & 0.31 \\
$v_{23}$ & 0.44 \\
$G_{I c}=G_{c, n}\left(\mathrm{~J} / \mathrm{m}^{2}\right)$ & 137 \\
$G_{I I c}=G_{c, t}\left(\mathrm{~J} / \mathrm{m}^{2}\right)$ & 502 \\
\hline
\end{tabular}


An OMAX 2626 Jet Machining Center (OMAX Corp. Kent, WA, USA) was used to perform the experiments. The AWJ pierces were performed with the jet in air (i.e. unsubmerged), at a stand-off distance (SOD) of $1 \mathrm{~mm}$.

Table 3.2 shows the operating pressures and nozzle sizes used in the piercing experiments. The AWJ power, $P_{o}(\mathrm{~W})$, of Table 3.2 was calculated using [3]

$$
P_{o}=P \times \frac{Q}{60}
$$

where $P(\mathrm{MPa})$ is the pump pressure, and $Q(\mathrm{~L} / \mathrm{min})$ is the mass flow rate. The mass flow rate was approximated using Bernoulli’s equation [3]

$$
Q=C_{d} A_{o} \sqrt{2 P / \rho}
$$

where $C_{d}$ is the discharge coefficient of the orifice, and $A_{o}$ is the orifice area. The discharge coefficient was approximated by [137]

$$
C_{d}=0.785-0.00014 P-0.197 d
$$

where $d$ is the diameter of the orifice in mm. The pump pressure dictates the velocity of the waterjet, which governs the magnitude of the hydraulic shock as seen in equation (3.1). Equations (3.2) - (3.3) show that the size of orifice contributes to the power of the jet, and thus its impact energy.

Based on video recordings (240 fps) of the jet at $150 \mathrm{MPa}$ using the $254 \mu \mathrm{m}$ nozzle, the measured piercing duration (less than about $0.1 \mathrm{~s}$ ) was much smaller than the time required to develop adequate abrasive entrainment ( $\sim 2 s)$. Therefore, a water-only jet was used in all the experiments. The time between the pneumatic valve opening and closing during the piercing experiments was $5 \mathrm{~s}$. The waterjet velocities and vena-contracta of the orifice inlet were estimated using the 1-D CFD model developed by Narayanan et al. [71], which has been shown to predict axial flow velocities exiting an AWJ nozzle within 5\%. 
Table 3.2 Experimental conditions for the piercing experiments.

\begin{tabular}{lllllll}
\hline Specimen & $\begin{array}{l}\text { Pump } \\
\text { pressure } \\
{[\mathbf{M P a}]}\end{array}$ & $\begin{array}{l}\text { Mixing } \\
\text { tube size } \\
{[\boldsymbol{\mu m}]}\end{array}$ & $\begin{array}{l}\text { Exit water } \\
\text { velocity }[\mathbf{m} / \mathbf{s}]\end{array}$ & $\begin{array}{l}\text { Orifice } \\
\text { dia. }[\boldsymbol{\mu m}]\end{array}$ & $\begin{array}{l}\text { Jet diam. } \\
{[\boldsymbol{\mu m}]}\end{array}$ & $\begin{array}{l}\text { AWJ } \\
\text { power } \\
{[\mathbf{k W}]}\end{array}$ \\
\hline 1 & 150 & 254 & 536 & 127 & 108 & 1.02 \\
2 & 200 & 254 & 618 & 127 & 108 & 1.58 \\
3 & 250 & 254 & 685 & 127 & 108 & 2.19 \\
4 & 150 & 381 & 536 & 178 & 151 & 4.07 \\
5 & 200 & 381 & 618 & 178 & 151 & 6.26 \\
6 & 250 & 381 & 685 & 178 & 151 & 8.68 \\
\hline
\end{tabular}

The samples were cut into $20 \mathrm{~mm} \times 20 \mathrm{~mm}$ square pieces from a $0.75 \times 0.5 \mathrm{~m}$ CF-epoxy sheet using the AWJ. These dimensions were dictated by the size of microCT scanner (Section 2.2). The surfaces were dried prior to piercing to remove any water films [138]. One specimen was used for each of the six conditions.

\subsubsection{Quantification of impact delamination}

The laminate pierces were analyzed using 3D x-ray micro-tomography (Skyscan 1172, Bruker Corp., Billerica, MA, USA) with a $0.5 \mu \mathrm{m}$ resolution. The raw data from the CT scans were analyzed using NRecon (version 1.6.5.0, Bruker Corp., Billerica, MA, USA) and opensource image analysis software, ImageJ. Due to the variation in the longitudinal and transverse elastic moduli of the CF-epoxy laminate, the delamination generated an elliptical damage zone in the $x y$-plane as illustrated in Fig. 3.2, which is typical of bi-directional laminate impacts [121, 139].

Since the focus of the present work was the damage created by the initial impact of the jet, only the delamination cracks in the vicinity of the top ply were measured in the longitudinal $\left(L_{D}\right)$ 
and transverse directions $\left(T_{D}\right)$, parallel to the ellipse major and minor axes, respectively. Due to the variation in nozzle size, the elliptical damage area $\left(D_{a}\right)$ was calculated by subtracting the circular area of the pierced hole of radius $R_{H}$ from the elliptical damage zone, and defined as,

$$
D_{a}=\pi L_{D} T_{D}-\pi R_{H}^{2}
$$

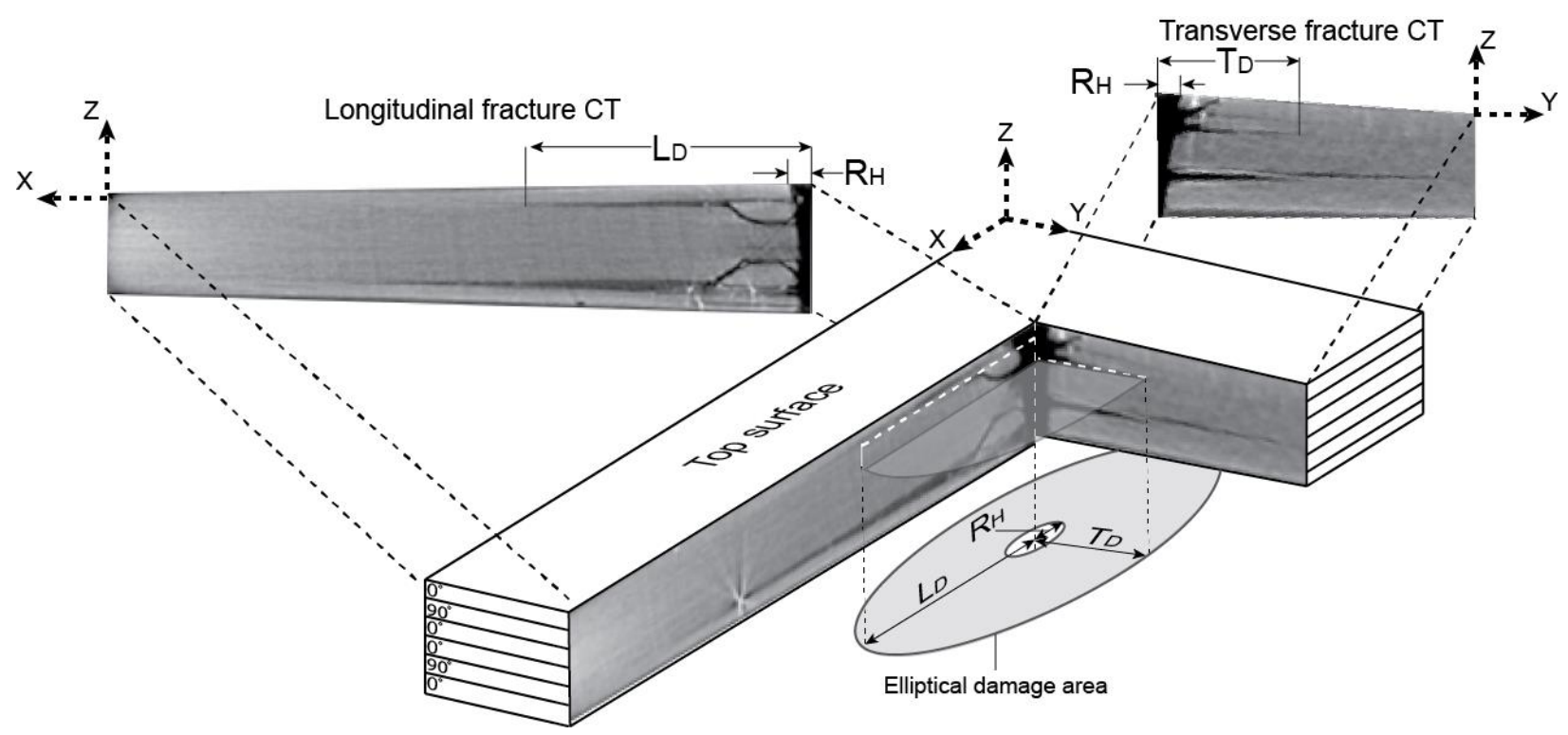

Fig. 3.2 Cross-sections of pierced laminate taken through the major and minor axes of the elliptical damage zone. $0^{\circ}$ plies had C-fibers that were parallel to the $x$-axis.

Large cracks were also observed in the bottom plies, but these were attributed to ply bending as the jet entered the final stages of piercing the laminate [44, 45]. Hence, bottom ply damage was assumed to be unaffected by the initial stages of the jet impact and was not considered in the present analyses.

\subsection{Modelling}




\subsubsection{Impact delamination}

\subsubsection{Model overview}

A fluid-structure interaction (FSI) model was developed using a 2-way finite-element model (FEM)/computational fluid dynamics (CFD) coupled analysis in ANSYS 15 (ANSYS Inc., Cecil Township, PA, USA). The structural and fluid domains and their respective boundary conditions are shown in Fig. 3.3. For each time-step of the analyses, the forces from the CFD model were shared with the structural FEM domain, and acted as the applied loading. The displacements were then calculated in the structural model and transferred back to the CFD model, resulting in an altered mesh for the following time-step. The impact time varied with the jet pressure (Table 3.2), so the simulation times were set to $2.5 \mu \mathrm{s}, 2.25 \mu \mathrm{s}$, and $2 \mu \mathrm{s}$, for the 150 , 200 and $250 \mathrm{MPa}$ cases, respectively. A general guideline for shock simulations is to ensure that the control volume diameter is at least 5-6 times the characteristic length (orifice radius in this case) in order to observe the effects of the initial wave propagation prior to the interference of rebounding waves from free and boundary surfaces [120]. Thus, a quartered cylindrical control volume was used with a radius of $2 \mathrm{~mm}$. 


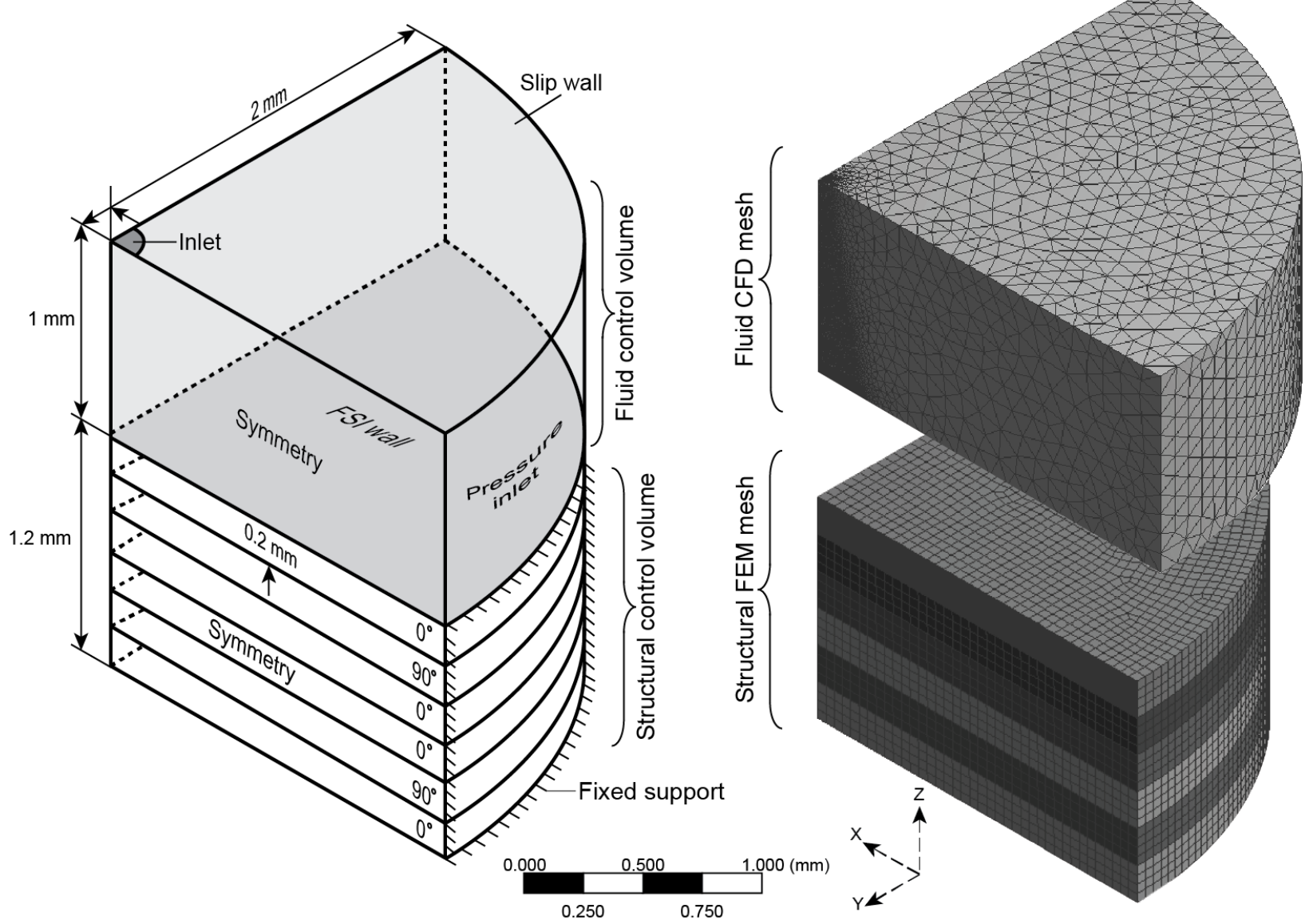

Fig. 3.3 CFD and structural control volumes used in the FSI model.

\subsubsection{CFD model}

Previous simulations have considered the waterjet as a two-phase flow of water with entrained particles [140]. In the present work, the fluid domain did not contain abrasive particles because, as explained in Section 3.1, they are absent during the generation of the shock wave that causes the initial damage. Although previous authors have successfully modelled AWJ flow exiting the nozzle using axi-symmetric simulations [140], the anisotropic behavior of the target composite material required the use of a three-dimensional model. Therefore, the CFD flow domain was quartered using symmetry boundary conditions. This methodology is valid considering the composite had a symmetric layup, resulting in symmetrical stress distributions in the longitudinal and transverse directions. The waterjet flow domain was solved using ANSYS Fluent 15.0 (Ansys Inc., Cecil Township, PA, USA). A multiphase, transient, volume-of-fluid 
(VOF), $k-\varepsilon$ shear-stress transport turbulence model was used for the waterjet surrounded by air $[108,141]$. The transient simulation used a 2 ns time step, which has been shown to be adequate to capture the hydraulic shock action [114].

The boundary conditions and flow domain of the CFD follow that of Lui et al. [140], with a slip wall adjacent to the velocity inlet and pressure inlets at the domain outflows. The shared wall of the structural/fluid domain was set as a stationary wall, but specified with a dynamic (deformable) mesh to account for substrate deformations occurring during impact. The waterjet velocities for the inlet boundary condition were estimated using the 1-D CFD model developed by Narayanan et al. [71], based on the mixing tube exit, rather than the orifice, to account for momentum considerations when accelerating the air inside the mixing tube during initial firing of the jet. The diameter of the inlet was based on the vena contracta of the waterjet nozzle orifices (Table 3.2), rather than the diameter of the mixing tube and was approximated based on the work of Corey et al. [142]. Basing the inlet diameter on the mixing tube diameter would lead to unrealistic mass flow rates for the nozzles. The fluid velocity distribution profile of the inlet boundary was modeled using a $1 / 7^{\text {th }}$ power law function via user defined sub-routines, based on the work of Wang et al. [126]. The CFD mesh was refined locally in the region from the inlet boundary to the substrate impact zone (1 mm standoff distance, Fig. 3.3) in order resolve the large pressure gradients associated with high-speed liquid jets. The meshes for the 254 and 381 $\mu \mathrm{m}$ nozzles consisted of $1.65 \times 10^{6}$ and $1.925 \times 10^{6}$ tetrahedral elements, respectively, with a convergence residual of $10^{-4}$, which was found to be appropriate in other CFD models of impinging jets [44]. A grid convergence index $(G C I=0.048)$ was calculated over three mesh sizes $\left(1.5 \times 10^{6}, 1.0 \times 10^{6}\right.$, and $\left.6.7 \times 10^{5}\right)$, and based on the maximum water hammer pressures for specimen 1 in Table 3.2. The convergence study showed that the results were independent of the element size, with tetrahedral elements producing the highest quality mesh compared to other methods for the given geometry.

Following the AWJ modeling work of Liu et al. [140], and Li et al. [99] for compressibility of liquid drop impact (LDI), a multi-phase VOF model was used. The VOF model is capable of simulating free surface jet flows between various types of fluids, both liquid and gas [141], where air and water were treated as the primary and secondary phases, respectively. The density 
$(\rho)$ and viscosity $(\mu)$ of the mixture are expressed as phase properties of the liquid volume fraction $\left(\alpha_{1}\right)$, which is the quotient of liquid volume over the total volume of the cell [99].

$$
\begin{aligned}
& \rho=\rho_{G}\left(1-\alpha_{L}\right)+\rho_{L} \alpha_{L} \\
& \mu=\mu_{G}\left(1-\alpha_{L}\right)+\mu_{L} \alpha_{L}
\end{aligned}
$$

The subscripts $G$ and $L$ represent the gas and liquid phases, respectively. The VOF gradient between the water and air interface was calculated using a higher-order compressive scheme (i.e. the compressive interface capturing scheme for arbitrary meshes - CICSAM [143]), consistent with the approach of Li et al. [99] for high-speed liquid drop impact.

Similar to the AWJ models of Liu et al. [140], the standard $k-\varepsilon$ turbulence model constants (Table 3.3) were taken from Launder and Spaulding [144]. The compressibility of the liquid jet upon impact was solved via the Tait equation using the parameters shown in Table 3.3. Such a model has been shown to be appropriate for calculating the release waves in high pressure $(<2.5 \mathrm{GPa})$ water [99].

Table 3.3. Standard $k-\epsilon$ turbulence model [144] and Tait equation parameters [99].

\begin{tabular}{lll}
\hline Model & Parameters & Values \\
\hline Standard $k-\epsilon$ & Constants & $C_{1 \epsilon}=1.44$ \\
turbulence & & $C_{2 \epsilon}=1.92$ \\
model & & $C_{\mu}=0.09$ \\
& Turbulent Prandtl & $\sigma_{k}=1$ \\
& numbers & $\sigma_{\epsilon}=1.3$ \\
\hline Tait equation & Reference pressure & $p_{o}=101.3 \mathrm{kPa}$ \\
& Reference density & $\rho_{o}=1000 \frac{\mathrm{kg}}{\mathrm{m}^{3}}$ \\
& Reference bulk & $K_{o}=2.2 \mathrm{GPa}$ \\
& modulus & \\
& Density exponent & $n=7.15$ \\
\hline
\end{tabular}

Heymann [107] postulated that the speed of sound is not constant during impact, but a function of the pressure. Thus, the speed of sound function and equation of state were implemented into the model via subroutines in order to stabilize the solution during compression. The speed of sound $(C)$ needed for pressure correction was defined as [99], 


$$
c=\sqrt{\frac{d p}{d \rho}}=\sqrt{\frac{K_{o}}{\rho}}
$$

\subsubsection{CFD model validation}

The CFD modelling approach was validated by comparing with the work of Bourne et al. [145], who recorded the jet impact pressures on a PMMA substrate using a piezoelectric sensor [146]. The CFD domain used in the present paper replicated the experimental geometry of Bourne et al. [145] with the exception that $\mathrm{SOD}=1 \mathrm{~mm}$ was used, whereas it was $15 \mathrm{~mm}$ in Bourne et al. [145]. Nevertheless, another work of Bourne [124] modelled the same experimental results under a reduced SOD and obtained similar results. The inlet boundary diameter of the model was based on the vena-contracta diameter of the jet exiting a nozzle and was approximated using the work of [142] as

$$
d_{j} \approx d \sqrt{C_{d}}
$$

where $d_{j}=1.07 \mathrm{~mm}$ was the vena-contracta diameter of the jet, and $C_{d}=0.45$ was approximated by Hashish [137]. Bourne et al. [145] only provided waterjet velocity, thus, the operating pressure required by equation (3.4) was approximated using Bernoulli's equation which has been shown to be in good agreement with the waterjet velocity exiting the orifice [79].

Fig. 3.4 shows that the measured [49] and CFD predicted pressures are in good agreement, and the model is capable of accurately predicting the water hammer brought on by the liquid jet impact. Although the model used for validation is different than that used in the composite modeling of the present work, this result nevertheless lends confidence to the validity of the modeling approach. 


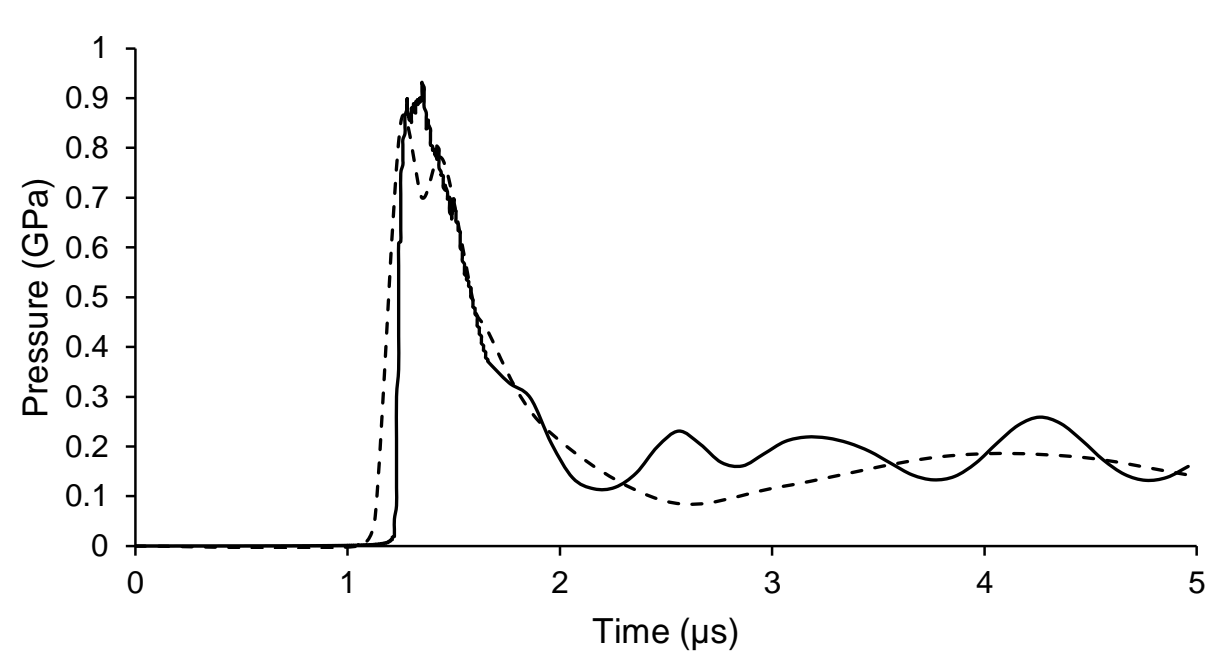

Fig. 3.4 Measured [145] and CFD predicted maximum impact pressure across the jet/substrate contact surface with respect to time.

\subsubsection{Structural FEM}

The structural simulation was conducted using ANSYS 15.0 Workbench (Ansys Inc., Cecil Township, PA, USA). The structural volume consisted of layered SOLID186 elements, a 20 node brick element typically used for 3D simulations using contact boundary conditions, using a reduced brick integration scheme, and meshed adaptively with a maximum element size of $25 \mu \mathrm{m}$ [147]. A convergence study showed that the results were independent of the element size. The orthotropic properties of the CF-epoxy lamina were based on the manufacturer provided specifications (Table 3.1). The CZM was implemented through the ANSYS "pure penalty formulation" using zero-thickness, 3D, surface-to-surface, pair-based contact elements (CONTA174 and TARGE170). A fracture-based debonding model was used to predict interlaminar crack propagation across the CZM contact elements. The contact elements were subject to interlaminar normal $\left(\sigma_{z}\right)$, and shear stresses $\left(\tau_{x z}\right.$ and $\left.\tau_{y z}\right)$, representing mode I, II and III crack propagation stresses, respectively [128]. The cohesive elements used a bi-linear, mixedmode, traction separation law, with the normal $\left(\sigma_{n}\right)$ and tangential $\left(\sigma_{t}\right)$ cohesive tractions expressed as [147],

$$
\sigma_{n}=K_{n} \delta_{n}\left(1-D_{m}\right)
$$




$$
\sigma_{t}=K_{t} \delta_{t}\left(1-D_{m}\right)
$$

where $K_{n}$ and $K_{t}$ are the normal and tangential stiffness, $\delta_{n}$ and $\delta_{t}$ are the normal and tangential displacements, and $D_{m}$ is the damage parameter associated with the CZM [147]. The contribution of the normal and tangential displacements on the interface separation were resolved by a nondimensional effective displacement $(\lambda)$ parameter [147]:

$$
\lambda=\sqrt{\left(\frac{\delta_{n}}{\delta_{n}^{c}}\right)^{2}+\beta_{d}^{2}\left(\frac{\delta_{t}}{\delta_{t}^{c}}\right)^{2}}
$$

where $\delta_{n}^{c}$ and $\delta_{t}^{c}$ are the normal and tangential displacements at the onset of debonding, and $\beta_{d}$ is a non-dimensional parameter set to 1 which enabled tangential slip of the contact elements under normal compressive stress.

The bi-linear CZM assumed that the normal (mode I) and tangential (mode II) contact stresses increased linearly to a maximum value $\left(\sigma_{c,(n, t)}\right)$, upon which damage was initiated. Beyond these critical stresses, the cohesive stress decreased until it reached a critical separation distance $\left(\delta_{c,(n, t)}\right)$. At this point, the cohesive stress vanished and debonding occurred. The area under the traction-separation curve (Fig. 3.5) was set equal to the critical strain-energy release rate $\left(G_{c,(n, t)}\right)$. The fracture envelope in the FE model was defined using a the widely-used criterion [147],

$$
\left(\frac{G_{n}}{G_{c n}}\right)^{2}+\left(\frac{G_{t}}{G_{c t}}\right)^{2}=1
$$

where $G_{n}$ and $G_{t}$ are the normal and tangential fracture energies. The normal and normal and tangential fracture energies were calculated via

$$
G_{n, t}=\int P_{n, t} d u_{n, t}
$$

where $P_{n, t}$ is the normal or tangential contact stress and $d u_{n, t}$ is the change in the normal/tangential contact gap. 


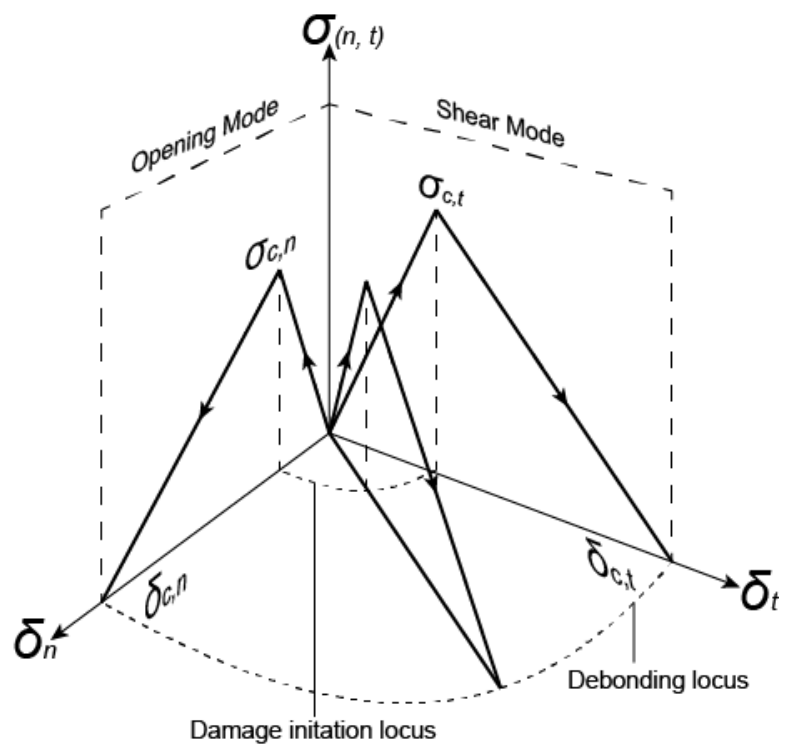

Fig. 3.5 Mixed-mode bi-linear, traction-separation relationship for cohesive elements [148].

The maximum normal $\left(\sigma_{c, n}=45 \mathrm{MPa}\right)$ and tangential $\left(\sigma_{c, t}=60 \mathrm{MPa}\right)$ contact stresses were an average approximation based on literature survey of ten CZM of carbon/epoxy laminates, with similar properites observed in the current laminate [149-158]. The initial stiffnesses of the interfaces $\left(K_{n}\right.$ and $\left.K_{t}\right)$ have been shown to have a negligible effect on the load predictions [159], thus both were set to $10^{14} \mathrm{~N} / \mathrm{m}^{3}$ following Refs. [160] and [161].

\subsection{Results and discussion}

\subsubsection{Impact modeling}

The model captured several phenomena that have been observed in previous research such as jet ablation $[112,125]$, shock front generation and shock detachment within the waterjet $[108,162]$, and stress wave propagation into the target [121]. These will be discussed in the next sections. 


\subsubsection{Development of jet front}

Fig. 3.6a shows the simulated AWJ at key time intervals as the jet travels in air across the SOD from the mixing tube exit, to the surface of specimen 1 in Table 3.2. The magnified images show the morphology of the jet front due to ablation during its flight.

\subsubsection{Development of hydraulic shock and interaction with substrate}

The frames of Fig. 3.6b show the water volume fraction (VF) contours (top-left of each time step), pressure (top-right of each time step) and equivalent elastic strain (EES) of the composite (bottom-right of each time step). Upon initial impact at $t=2.12 \mu \mathrm{s}$, the hydraulic shock

formed at the center of the jet due to the curved jet front and the compression of the fluid [115], and was transferred into the substrate. As the impact progressed $(t=2.13 \mu \mathrm{s})$, the jet contact area increased, while the shock front remained attached to the substrate surface. At $t=2.13 \mu \mathrm{s}$ the zone of maximum pressure had decreased and shifted radially outward and became isolated (solid arrow in $t=2.13 \mu \mathrm{s}$ frame), forming a circumferential band of high pressure as observed by Li et al. [99] for LDI impact and by Bowden and Brunton [112] for liquid jet impact. At $t=2.14 \mu \mathrm{s}$ the contact area of the jet and high-pressure region continued to expand, and lateral jetting had yet to occur so that the water remained in a high state of compression. Notably, the highest strain values were observed at this point. At time $t=2.16 \mu \mathrm{s}$, the pressure began to drop rapidly due to the initiation of the shock front detachment from the surface and the initial release of the compressed water as lateral jetting (solid arrow in $t=2.16 \mu \mathrm{s}$ frame) began. The jet and substrate now showed signs of a compression front disconnecting from the impact location (dashed arrows in $t=2.16 \mu \mathrm{s}$ frame). At $t=2.18 \mu \mathrm{s}$, the extent of lateral jetting increased (solid arrow in $t=2.18 \mu \mathrm{s}$ frame) and was marked by the continual decrease in pressure. The jet and substrate showed continual stress wave propagation into their respective interiors, with reducing pressure and strain values as a result of impact energy absorption. Finally, $t=2.20 \mu \mathrm{s}$ showed full lateral jetting (circle in $t=2.20 \mu \mathrm{s}$ frame) with a maximum pressure close to the stagnation pressure. The initial stress wave in the waterjet was now completely disconnected from the point of impact (solid 
arrow in $t=2.20 \mu \mathrm{s}$ frame) and was moving upward through the jet. These snapshots of the waterjet impact process reveal that it is a rapid and complex phenomena.

a.
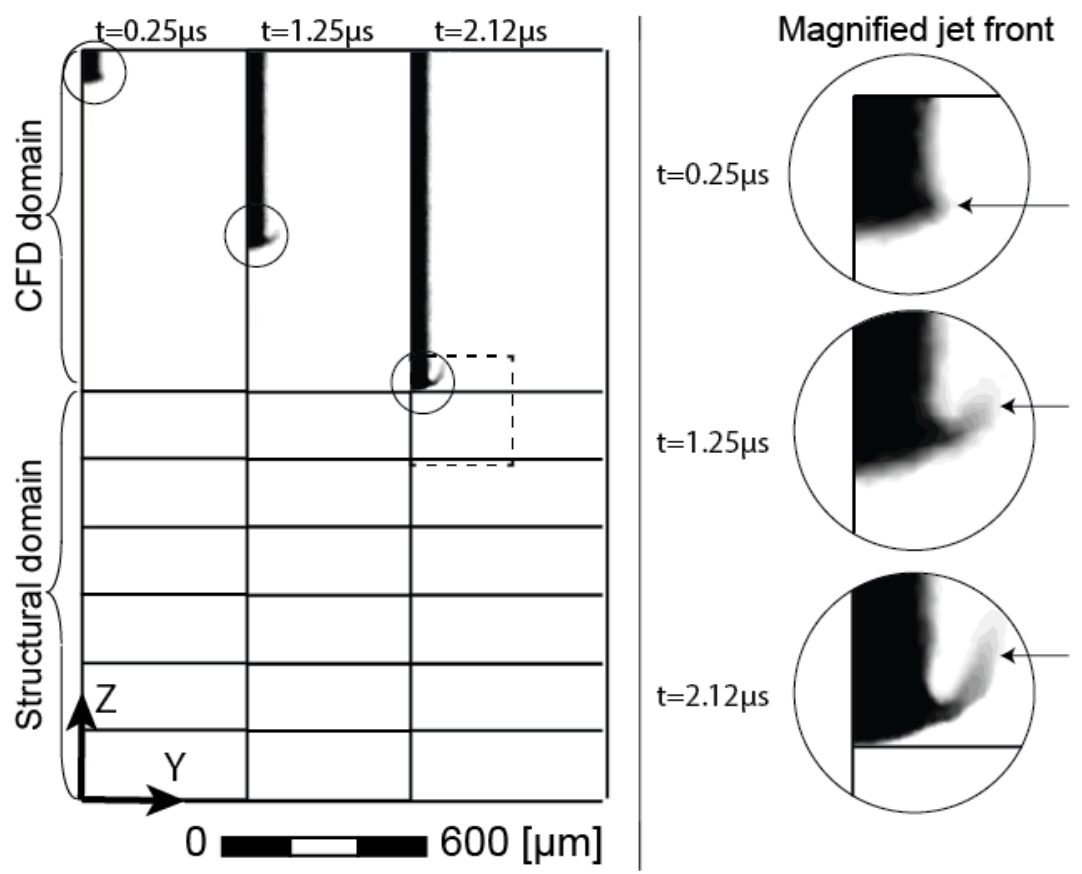

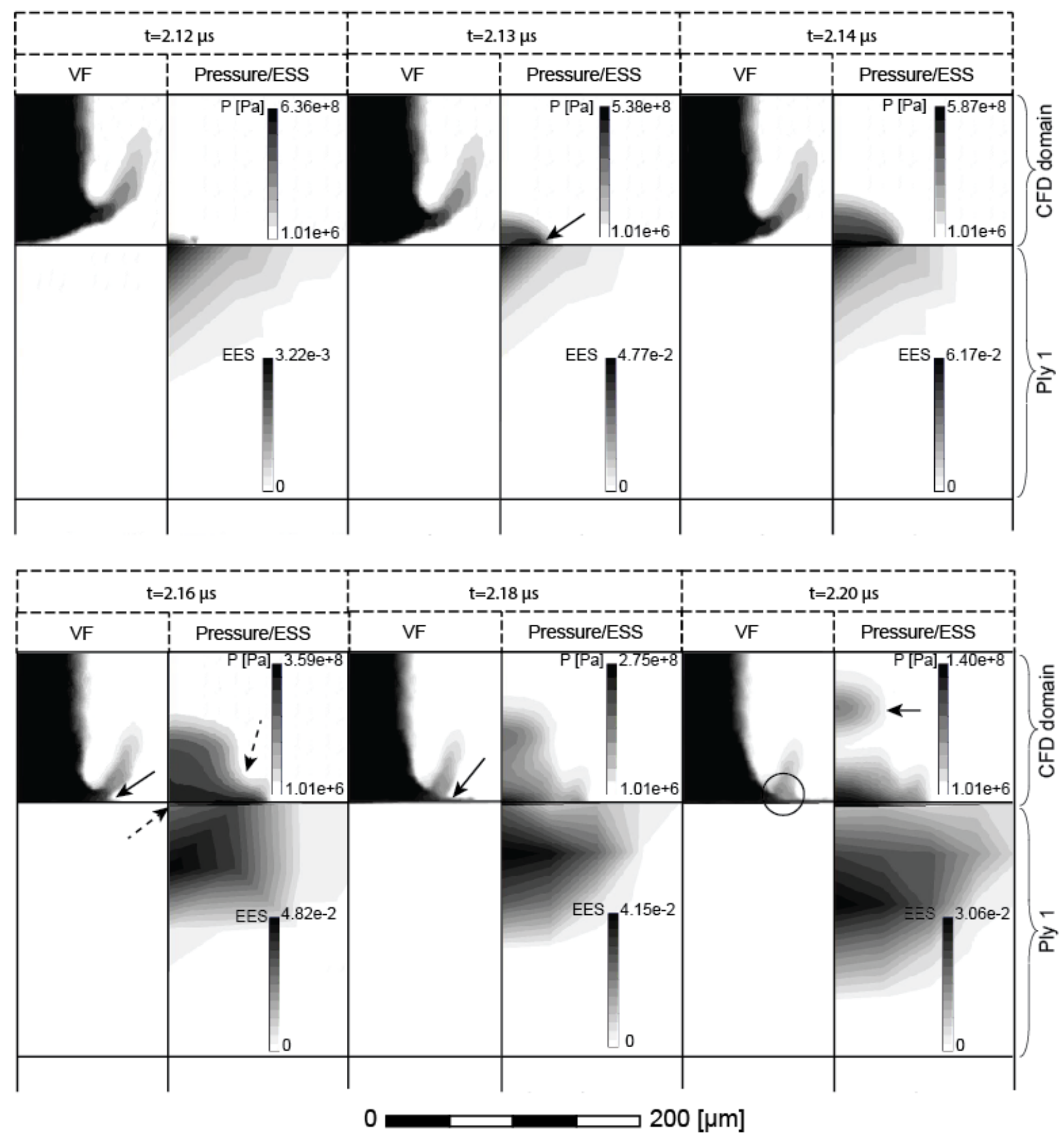

Fig. 3.6 FSI results of experiment 1 in Table 3.2 for: a. time-steps $0.25-2.12 \mu$ s showing the VF frames during its flight time across the SOD and corresponding to the $z y$-plane of the entire domain space as shown in Fig. 3.3 (circles show magnified views of the jet front), and, b. magnified results (corresponding to the region within the dashed box in a.) from time-steps $\mathrm{t}=$ $2.12-2.20 \mu$ s showing the hydraulic shock action. The VF frames of each time step show the shape and morphology of the waterjet during impact. Similarly, the Pressure/EES frames show the pressure generated inside the waterjet, and the equivalent elastic strain (ESS) response due to the waterjet loading, at each time step. 


\subsubsection{Hydraulic shock pressure and duration}

Fig. 3.7 shows that the maximum pressures predicted along the contact areas followed trends that are typical of hydraulic shock, with a rapid increase in pressure, followed by a more gradual decrease to the stagnation value [127]. As expected, higher jet velocities and larger nozzles (as shown in Table 3.2) generated greater hydraulic shock pressures.

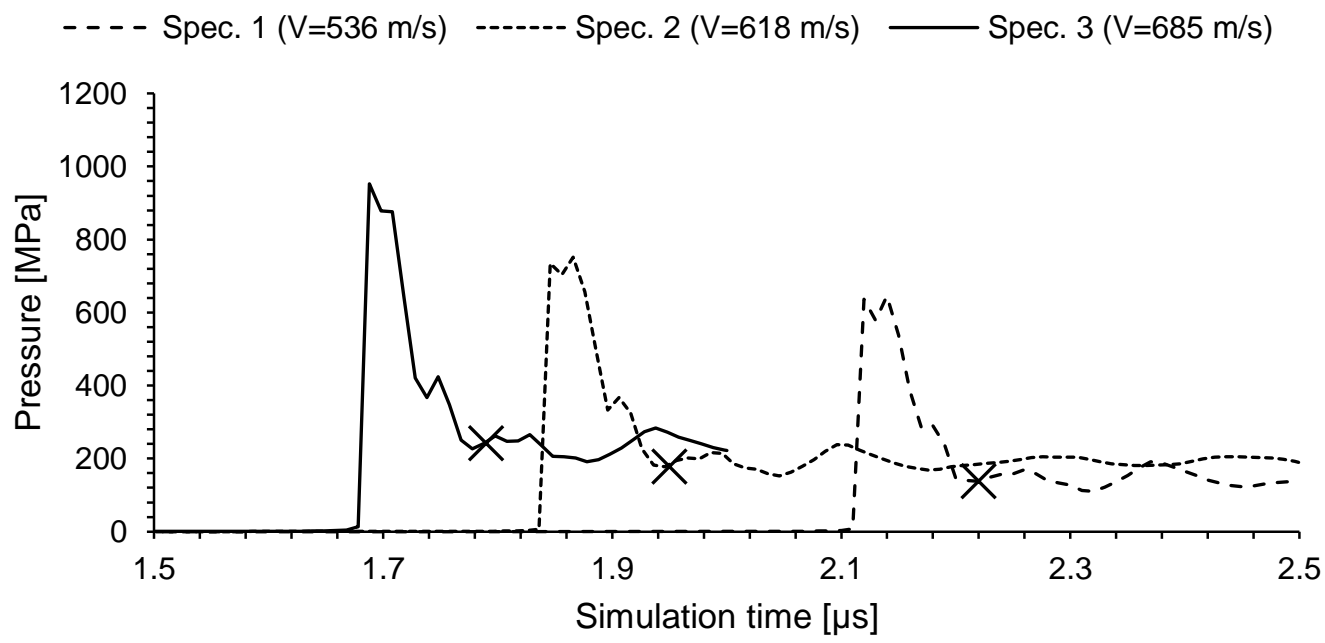

a. - - - - Spec. $4(\mathrm{~V}=536 \mathrm{~m} / \mathrm{s})$------ Spec. $5(\mathrm{~V}=618 \mathrm{~m} / \mathrm{s}) \quad$ - Spec. $6(\mathrm{~V}=685 \mathrm{~m} / \mathrm{s})$

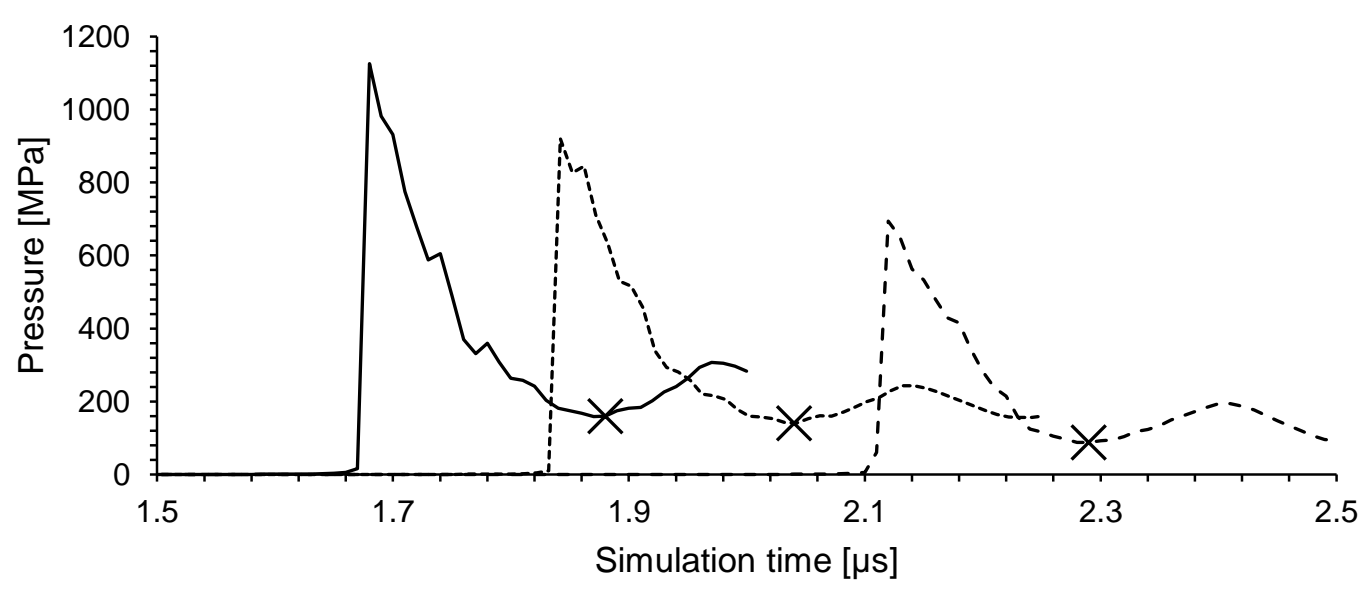

b.

Fig. 3.7 CFD results of the maximum pressure exerted on the substrate for: (a) the $254 \mu \mathrm{m}$ nozzle and (b) the $381 \mu \mathrm{m}$ nozzle. ' $\mathrm{X}$ ' marks the endpoint of the hydraulic shock, which is defined later in the current section. 
Fig. 3.8 compares the maximum hydraulic shock pressures predicted by the CFD with those of equation (3.1), which increased with both jet velocity and size. The increased pressure due to increased velocity was consistent with the predictions of the water hammer equation of Cook [100], with an average error of $12 \%$. However, the one-dimensional theory used by Cook does not consider the elastic response of the substrate, which has been shown to reduce the hydraulic shock action [107].

The approximation of water hammer pressure on an elastic substrate is given by [107],

$$
p_{s}=\frac{\rho_{w} c_{w}}{1+\left(\frac{\rho_{w} c_{w}}{\rho_{s} c_{s}}\right)} v
$$

where $\rho_{s}$ and $c_{s}$ are the density and acoustic wave velocity of the substrate. However, Heymanns' [107] approximation is only valid for isotropic materials since it assumes a constant density and wave velocity. The non-homogenous structure and directionally dependent wave velocities of the CF-epoxy violate these assumptions. Nevertheless, a single value of wave speed for the CF-epoxy can be approximated using the weight fraction of fibers and matrix, respectively (i.e. $c_{s, \text { ave }}=10.8(0.6)+3.04(0.4)=7.7 \mathrm{~km} / \mathrm{s}$ ), and the average density shown in Table 3.1. It is noted that wave speed depends on directionality and stack layup, of which the above approximation neglects. With these effective properties equation (3.14) better approximated the maximum pressure with an average error of $10 \%$ (Fig. 3.8).

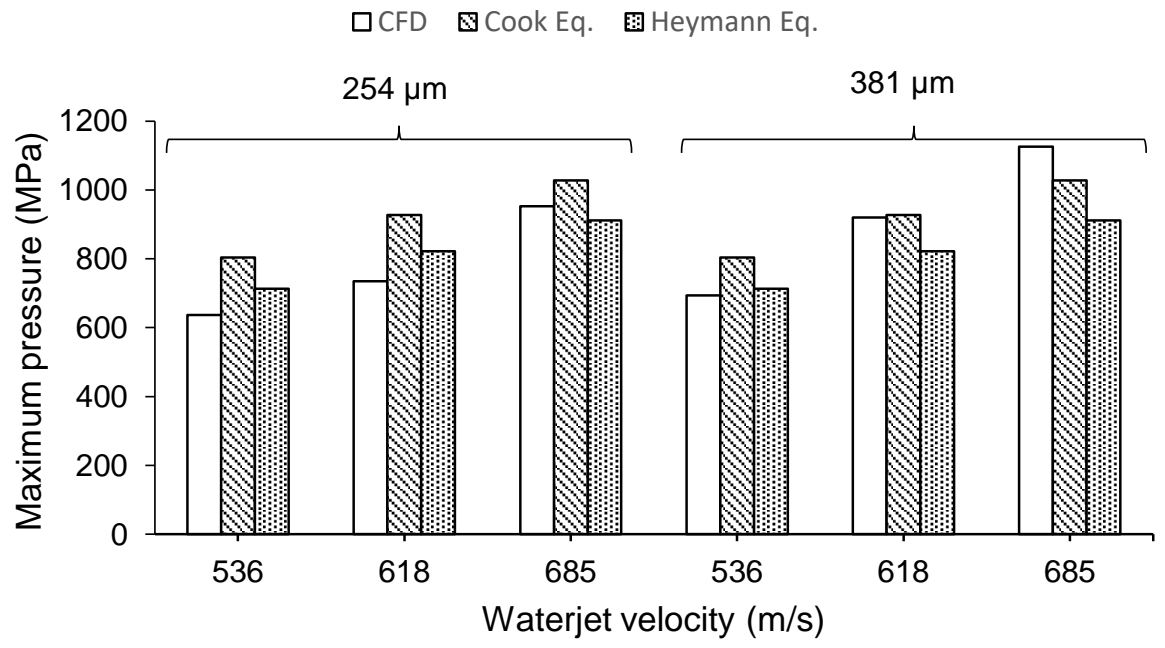

Fig. 3.8 Maximum hydraulic shock pressures predicted by CFD model and water hammer equations of Cook (equation 3.1)[100], and Heymann (equation 3.14)[107]. 
The trend of increased pressure with increased jet diameter was primarily attributed to the prolonged duration of the hydraulic shock action. The CFD predicted durations of the hydraulic shocks, defined from the instant the jet first contacted the substrate to the global minimum after the maximum pressure, as identified by an ' $X$ ' in Fig. 3.7. The average water hammer durations were $113 \pm 5 \mathrm{~ns}$ and $210 \pm 14 \mathrm{~ns}$, for the $254 \mu \mathrm{m}$ and $381 \mu \mathrm{m}$ nozzles, respectively. The increased duration for larger jets can be partially attributed to the increased contact area prior to the detachment of the shock front [114]. It can also be partially attributed to the $1 / 7^{\text {th }}$ power law shape of the jet exiting the nozzle.

Fig. 3.9 compares the predicted axial velocity distribution in the jet fronts exiting the nozzle for the $254 \mu \mathrm{m}$ and $381 \mu \mathrm{m}$ nozzles, for a pressure of $275 \mathrm{MPa}$. The initial contact angle of the jet front (i.e. the apex of the profiles) was shallower for the $381 \mu \mathrm{m}$ mixing tube, compared to the $254 \mu \mathrm{m}$ nozzle. Thus, for the same velocity, the contact angle of the larger nozzle would be reduced during impact, prolonging the time to reach the critical contact angle where the shock detaches from the substrate (i.e. $t=2.16 \mu$ s in Fig. 3.6). This implies that nozzle diameter influences the water hammer action, and might be optimized to reduce the influence of hydraulic shock. The influences of these phenomena on shock induced damage will be discussed in Section 3.4.2.2.

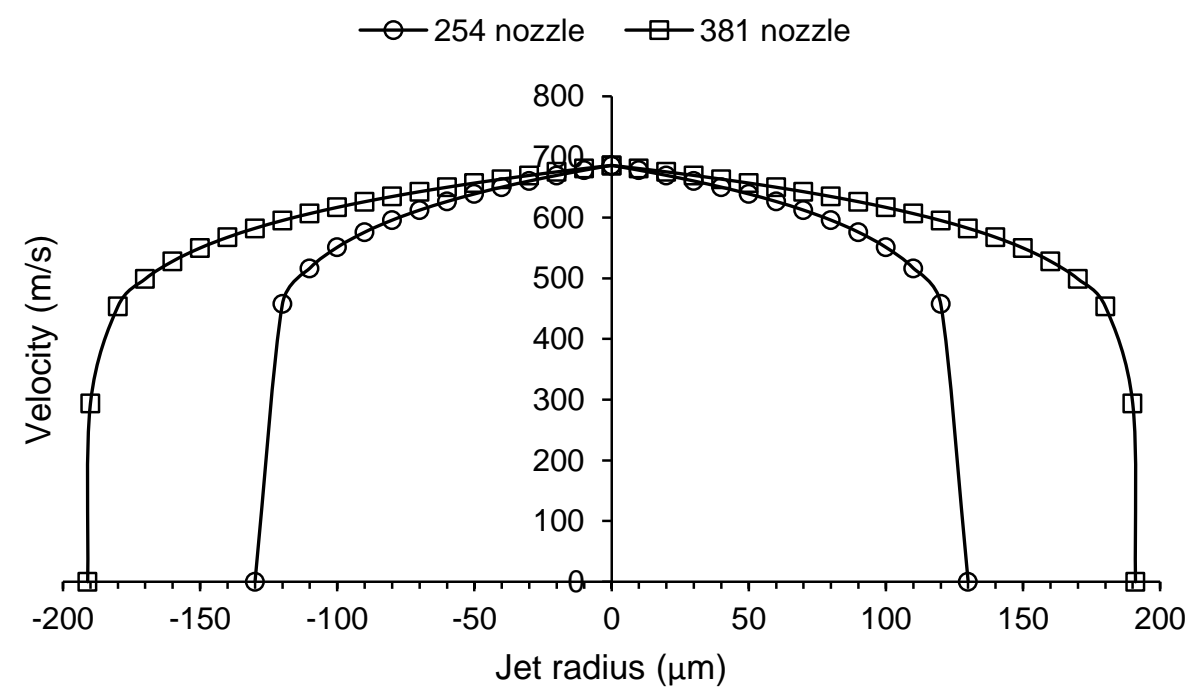


Fig. 3.9 Jet front axial velocity profiles as a function of mixing tube radius.

\subsubsection{AWJ admittance of the CF-epoxy substrate}

Admittance is formally defined as the change in wall deformation velocity with respect to change in pressure, and has been shown to have an effect on water hammer loading [108]. Higher admittance (i.e. greater deformation given a constant impact time and pressure) results in greater shock detachment contact angles [109], allowing for delayed initiation of lateral jetting, and reduced hydraulic shock loading due to energy consumption by the elastic deformation of the target $[107,127]$. Consequently, the anisotropic deformation of the substrate (Fig. 3.11b) in response to the jet impact resulted in asymmetrical stress distributions inside the material and an unequal pressure distribution along the solid-liquid interface of the jet. This varying pressure distribution was attributed to the changing critical contact angles along the jet periphery. Fig. 3.10 compares the pressure distribution along the surface of an isotropic target (polyethylene), the present anisotropic target (CF-epoxy), and a fictitious isotropic material with elastic properties based on the weight fractions of the epoxy and carbon fiber of the CF-epoxy under otherwise identical conditions. Since the constituent properties of the CF-epoxy were not provided by the manufacturer, the C-fiber and epoxy moduli were approximated as $E_{11}$ and $E_{22}$, respectively, using the properties in Table 3.1 (e.g. $E_{c}=W_{f} E_{11}+\left(1-W_{f}\right) E_{22}=0.6(117)+$ $0.4(8)=73.4 \mathrm{GPa})$. It should be noted that a marginal error was introduced using $E_{11}$ and $E_{22}$ instead of the fiber and matrix moduli, which neglects the micro mechanism of stress transfer between the matrix and fibers. However, this assumption is valid considering the longitudinal and transverse moduli of a uni-directional ply is close in value to the fiber and matrix moduli, respectively. Except for the small asymmetry due to the use of an unstructured mesh, the distributions of the isotropic cases were close to symmetric, as expected. However, clear asymmetry can be seen in the anisotropic case as a result of the asymmetric deformation of the substrate surface under loading. This directional and time dependence of the loading in both the waterjet and anisotropic substrate thus justifies the use of the 3D coupled transient simulation methodology. As previously explained, the relative magnitudes of the maximum pressure shown in Fig. 3.10 increased for more rigid targets in the jet axial direction (i.e. polyethylene $E=1.1$ $\mathrm{GPa}, \mathrm{CF}$-epoxy $E_{33}=8 \mathrm{GPa}$, and $\left.E_{c}=73.4 \mathrm{GPa}\right)$. 


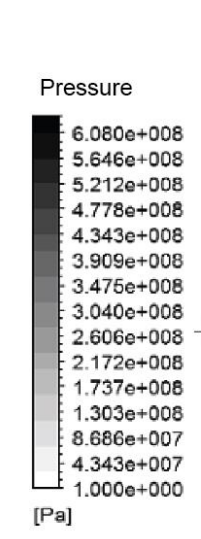

a.

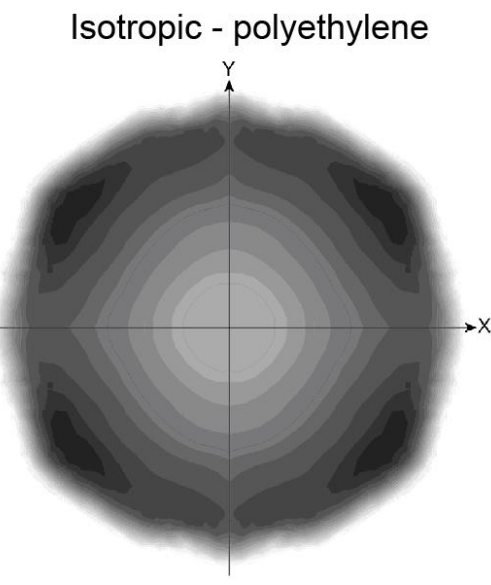

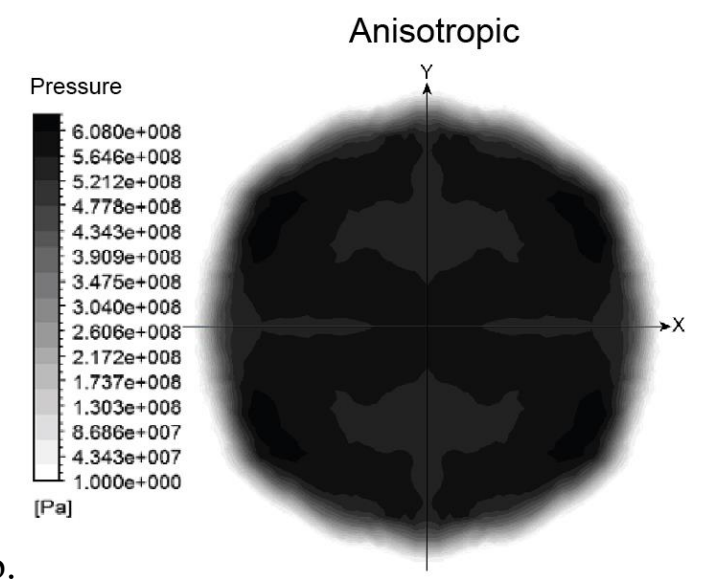

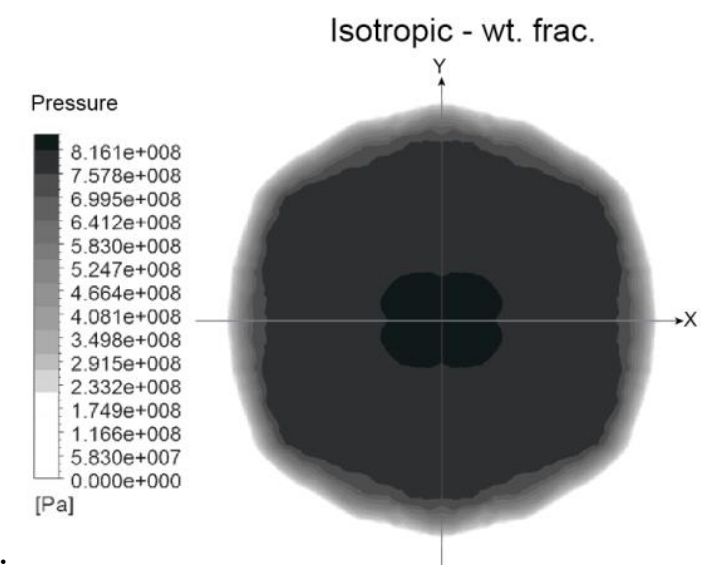

Fig. 3.10 Variation of impact pressure along the substrate surface when pressure was maximum for a. isotropic polyethylene, b. anisotropic model of the CF-epoxy, and c. isotropic model based on weight-fraction averaged moduli. Isotropic pressure contours generated using the same FE model as specimen 1 in Table 3.2, but with isotropic properties. Anisotropic pressure contour taken from specimen 1 in Table 3.2. Diameter of pressure zone was $104 \mu \mathrm{m}$.

Fig. 3.11 compares the target surface elevation at the time of maximum deformation of the isotropic polyethylene, anisotropic CF-epoxy, and the weight-averaged isotropic cases, acting along the centerline $x z$ and $y z$-planes, respectively. As expected, the deformation of the isotropic substrates were equal in the $x z$ and $y z$-planes, unlike that of the anisotropic composite. 


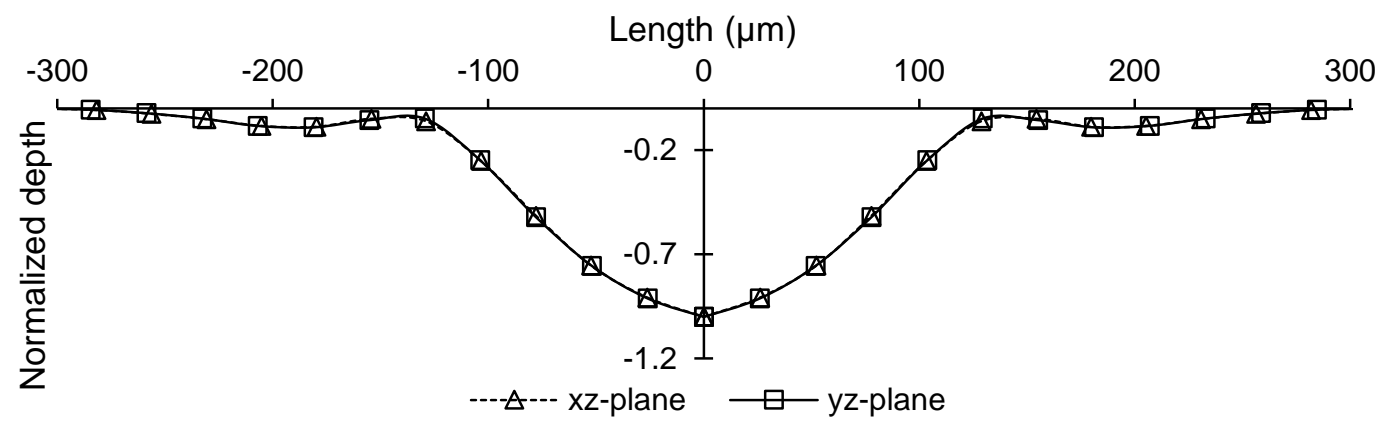

a.

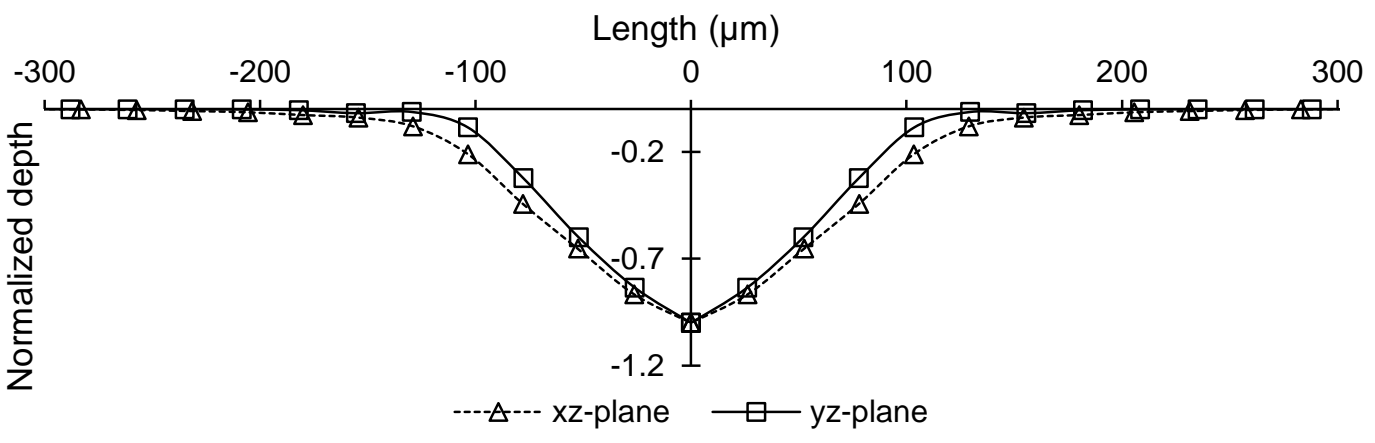

b.

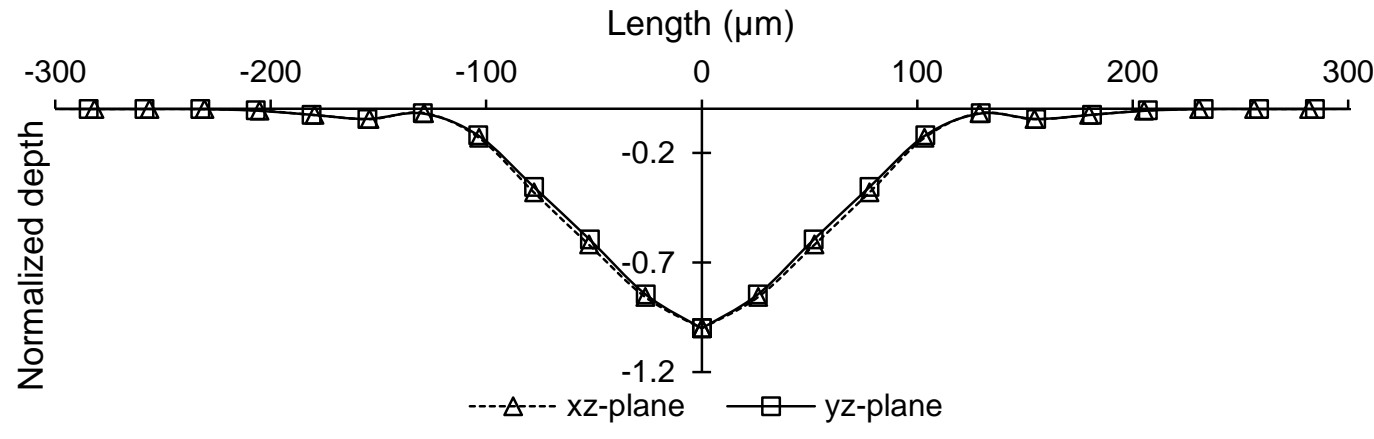

Fig. 3.11 Target surface deformation at maximum deflection for the a. isotropic (polyethylene), b. anisotropic (CF-epoxy), and c. weight-fraction averaged isotropic cases, along the centerline of the $x z$ and $y z$-planes, respectively.

\subsubsection{Damage in composite due to AWJ hydraulic shock}

\subsubsection{3D x-ray micro tomography}

Fig. 3.12 compares CT-scans of the $x z$-plane along the center of the pierced hole for specimens 1 and 6 in Table 3.2. Specimens 1 and 6 correspond to the minimum and maximum jet powers used in the experiments, respectively. The results show that, as expected, specimen 6 
had considerably more damage, with inter- and intra-laminar delamination occurring in all plies, whereas specimen 1 showing only inter-laminar damage along the bottom interfacial layer. The large delamination at the bottom of the hole was due to the bending of the last ply as the jet penetrated into it $[44,45]$, and was out of the scope of the present work which focuses on the interlaminar crack initiation on the top ply resulting from the initial AWJ impact. The increased delamination associated with specimen 6 (Fig. 3.12b) was attributed to the increased hydraulic shock loading and hydrodynamic pressurization associated with the more powerful jet. The white dots in the images are "beam hardening" artifacts due to the preferential attenuation of lowenergy x-rays, and do not represent changes in the laminate properties [163].

a.
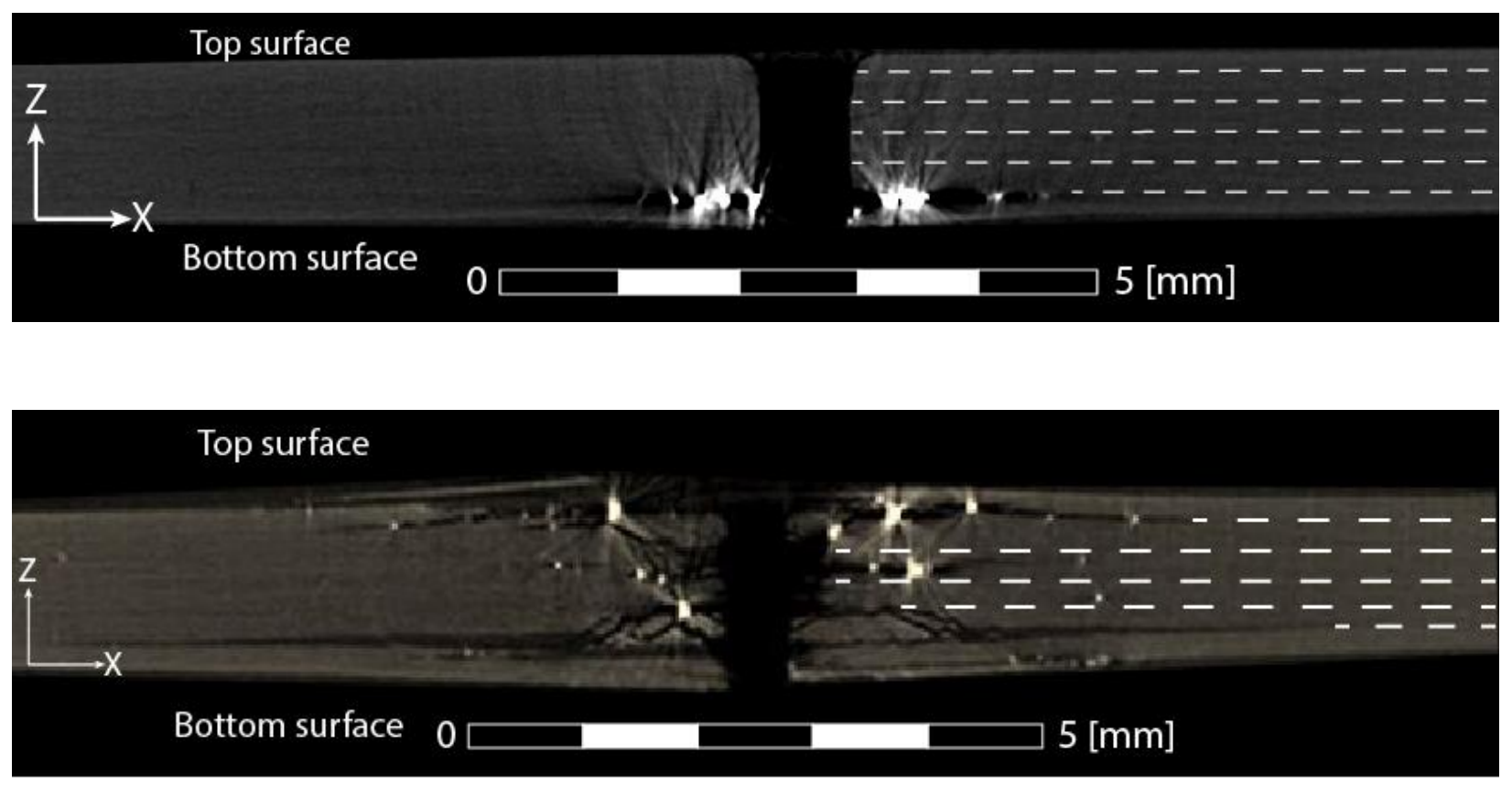

Fig. 3.12 X-ray micro-tomography scans along the centerline of the pierced holes along the $x z-$ plane for: a. specimen 1, and b. specimen 6 in Table 3.2. Dashed lines represent the lamina interfaces. Jet was incident from the top.

As shown in Fig. 3.2, delamination occurred in both the longitudinal and traverse directions during AWJ piercing. Across all specimens of Table 3.2, the elliptical damage zone had an average area of $31.1 \pm 18.8 \mathrm{~mm}^{2}$ ( \pm 1 standard deviation). The average longitudinal crack length was $3.39 \pm 1.90 \mathrm{~mm}$, which was $140 \%$ larger than the average transverse crack length of 
$2.39 \pm 1.35 \mathrm{~mm}$, as shown in Fig. 3.2. The average hole diameters for the 254 and $381 \mu \mathrm{m}$ nozzle were $0.92 \pm 0.10 \mathrm{~mm}$ and $1.01 \pm 0.12 \mathrm{~mm}$, respectively. The elliptical debonding pattern agreed with previous impact literature [121,164], with cracks extending further in the direction of higher elastic modulus [164].

Fig. 3.13 shows the effect of nozzle size and pressure on the measured elliptical damage zone from the micro-CT scans. As expected, increases in pressure and nozzle size resulted in increased delamination, due to the increased hydraulic shock loading associated with the increased impact energy of the jet. As discussed in Section 3.4.1.3, increased jet velocity (Fig. 3.8) and increased jet radius (Fig. 3.9) initiated larger subsurface defects. Likewise, the increased pressure and volumetric flow rate associated with faster and larger jets increase the propensity for further crack propagation due to hydrodynamic pressurization as the jet drilled through the target.

$\square 254 \mu \mathrm{m} \quad \approx 381 \mu \mathrm{m}$

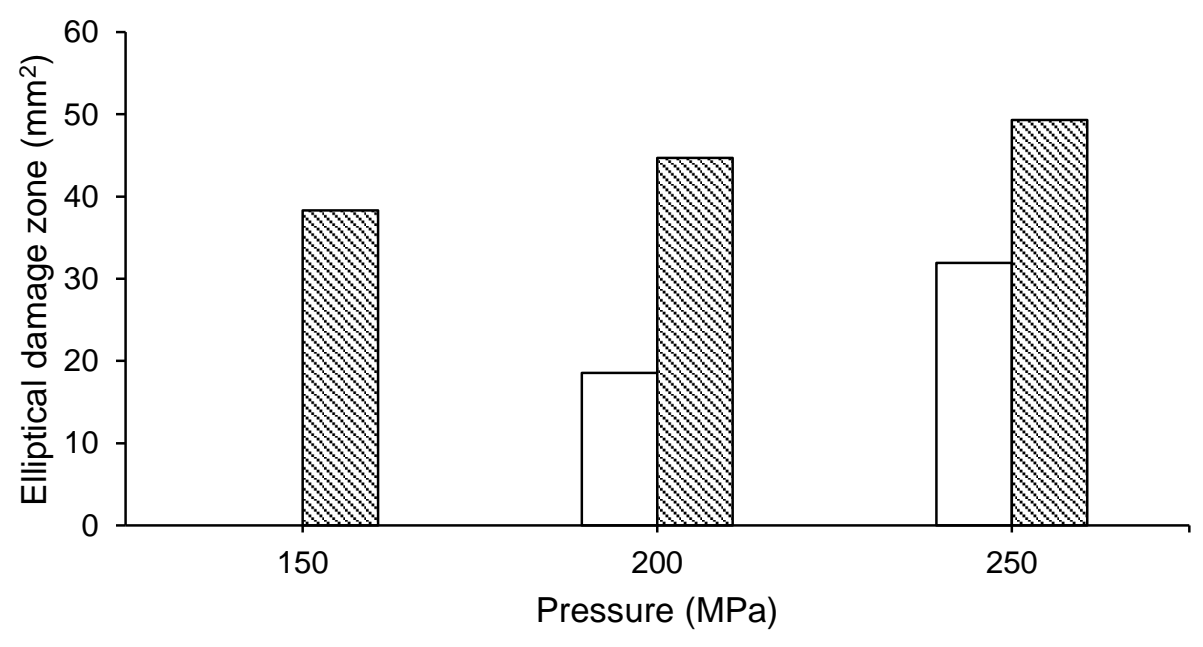

Fig. 3.13 Elliptical damage zone area in the top ply as a function of nozzle pressure for the two nozzle diameters.

As shown in equations (3.2) and (3.3), operating pressure and orifice diameter affect the power of the jet. Increased jet power results in greater impact energy absorption by the target. If the stress waves induced by the absorption of the impact energy exceed that of the damage threshold of the material, then deformation and/or crack initiation occurs in the substrate [120]. 
Fig. 3.14 shows that the measured elliptical damage zone increased with jet power, and that there may have been a critical piercing threshold energy below which no damage was created. It also indicates that the elliptical damage zone along the top ply tended toward a steady-state size with increased jet power. The results show that a threshold existed $(\sim 1 \mathrm{~kW})$ for the CF-epoxy, where delamination of the top ply due to piercing was eliminated.

\subsubsection{Damage prediction}

The impact energy of the water jet is absorbed by the target, and several stress waves (longitudinal, shear, Rayleigh) are propagated throughout the material [120,121]. Based on the anisotropic behavior of the present CF-epoxy, the impact energy absorption depended on the direction of wave propagation [122]. These stress waves were responsible for damage initiation and propagation within the material [121].

The present model did not simulate material removal, and was thus only valid for damage due to the propagation of the initial impact stress wave in the vicinity of the top ply. Similarly, the model did not consider intralaminar cracking, since the cohesive zone elements were restricted to the ply interfaces to model interlaminar delamination. This restriction was justified since it has been shown that there is a higher propensity for delamination along the ply interfaces during composite impact $[121,129,139]$ and abrasive waterjet machining [44, 84]. Since waves propagated in the CF-epoxy at far higher speeds than the drilling rate of the waterjet, crack initiation occurred prior to hydrodynamic pressurization which then propagated the delamination $[44,84]$. The present model predicted the location and size of the initial flaw generated by the waterjet impact, with the implication that larger flaw sizes result in greater hydrodynamic pressurization, and thus greater delamination.

Fig. 3.14 compares the predicted and micro-CT measured debonded areas. As discussed above, the model only predicted the initiation of debond and not its propagation, because that would require modeling of the pressurization of the initiated cracks. Nevertheless, when normalized by the highest value, the trends are very similar. This indicates that larger cracks initiated by the waterjet impulse ultimately resulted in greater hydrodynamic pressurization, and thus increased delamination. The increased crack size allows for the infiltrating pressurized 
water to act over a larger surface, generating increased loading and thus greater crack propagation.

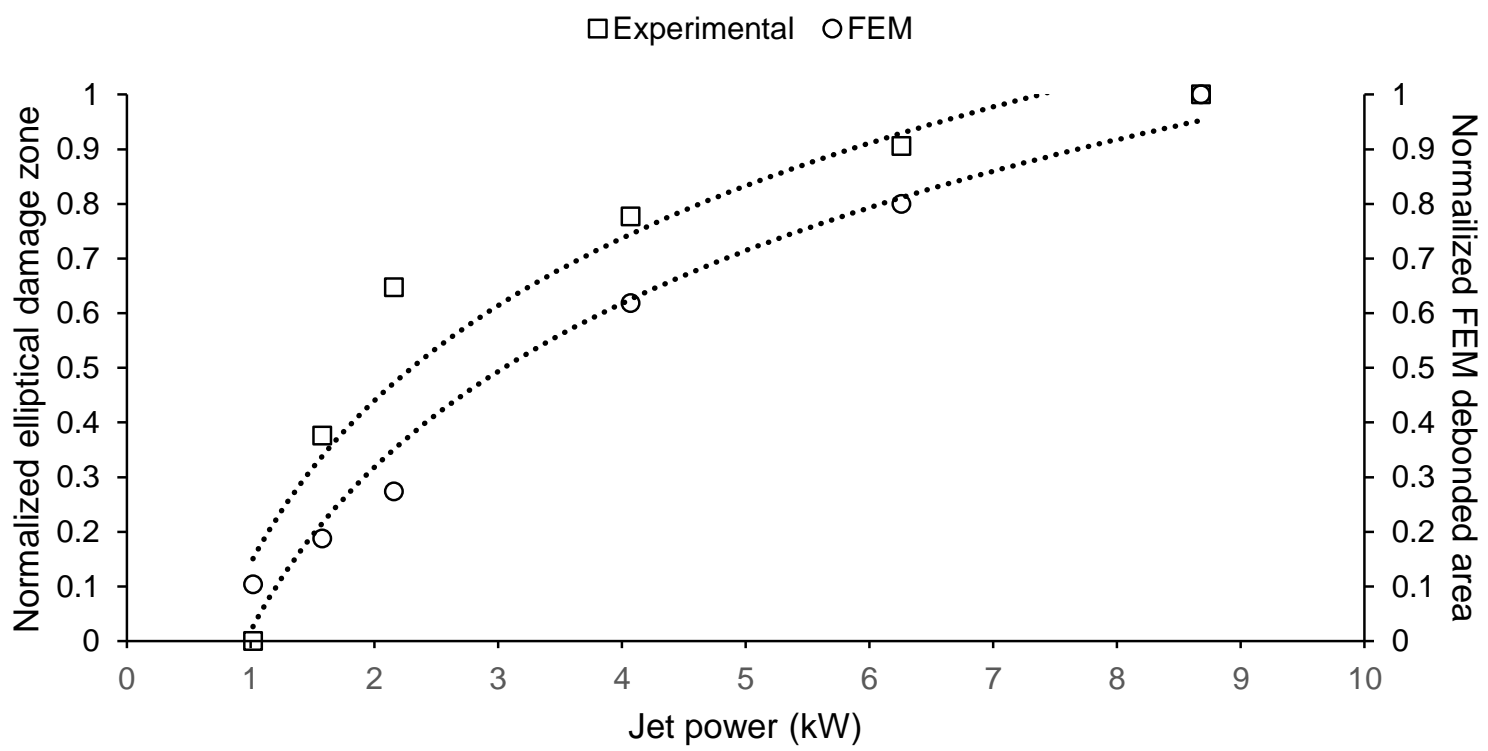

Fig. 3.14 Comparison of the influence of jet power on the measured top-ply elliptical damage zone (left axis) and FEM top-ply debonding area (right axis) (conditions of Table 3.2). Results normalized by maximum values. Trend lines are to guide the eye. No replicates were used in the experiments.

Fig. 3.15 shows the equivalent elastic strain (in the $y z$-plane) and debonding after the AWJ impact (in the $x y$-plane) at key time intervals. At $t=2.13 \mu \mathrm{s}$, the results show a high local deformation at the center of impact. This is consistent with the observations of Brunton [123], who has shown that plastically deforming materials will produce central depressions. Several authors have attempted to define the pressure distribution on targets [114, 165], however there is no consensus that fully explains the occurrence of all observed failure modes in the impact region (e.g. ring crater, cracks, and lip lines)[110]. The pressure plots of Fig. 3.10 show that the maximum loading of the jet impact on the present anisotropic target was non-uniform. Thus, the asymmetric surface loading interacted with the anisotropy properties of the composite to generate an irregular damage response in the substrate (Fig. 3.15). 
The release wave propagated radially from the point of impact (Fig. 3.15), losing intensity due to heat generation and wave attenuation [120]. The stress propagation into the substrate followed a similar trend as observed by Hsu. et al [110], Bourne [114] and Obara et al. [111] for jet impact on PMMA. At $t=2.25$, the stress wave reached the interface between plies 1 and 2, resulting in debonding of the CZM elements. The gap generated by the debonded region prohibited the transmission of stress waves to the second lamina, and thus restricted the wave motion to the generated free surfaces along the interface [120]. As the stress wave continued to propagate along the interface, the debonding area also increased until the stress wave had insufficient energy and crack propagation ceased. The FEM results in the last two frames of Fig. 3.15, which are magnified in Fig. 3.16, show that the debonding generated greater crack lengths in the longitudinal $(x)$ direction compared to the transverse $(y)$ direction. This correlated well with the experimental results shown in Fig. 3.2 and was another result of the targets' anisotropy. The model results show that increased pressure (Fig. 3.8) and nozzle size (Fig. 3.13) resulted in increased delamination. As discussed in Section 3.4.1.3, the increased pressure generated higher waterjet velocities, resulting in a larger hydraulic impact loading. Similarly, increased jet diameter prolonged the water hammer action, thus promoting increased delamination. This result reinforces the observation of Hashish [98] that smaller nozzles reduce impact delamination. 


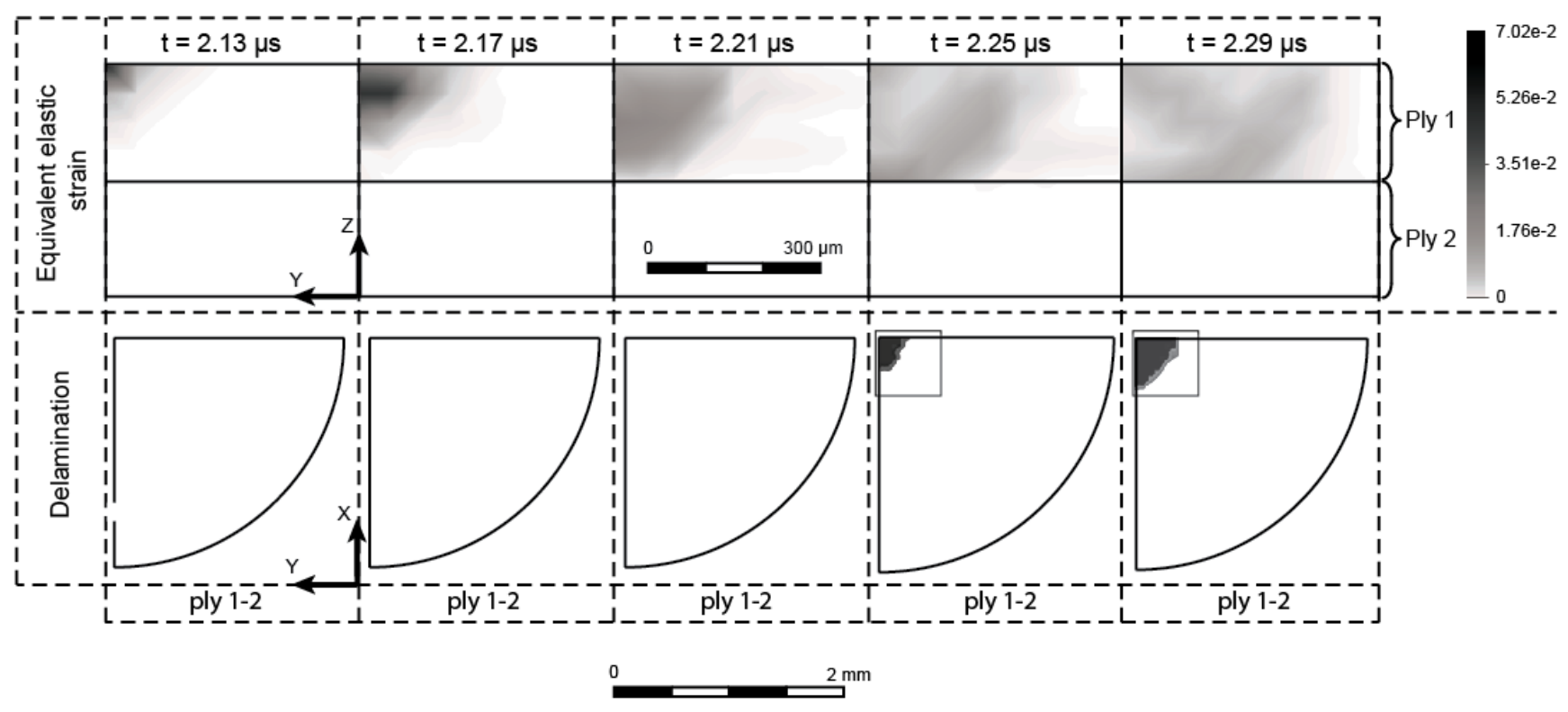

Fig. 3.15 Predicted equivalent elastic strain (top row in the $y z$-plane normal to the target surface) and debonding (bottom row in the $x y$-plane of the target surface) at key time steps during the AWJ impact simulation zoomed to plies 1 and 2. Debonding is shown as the dark areas in the delamination row.

Fully debonded
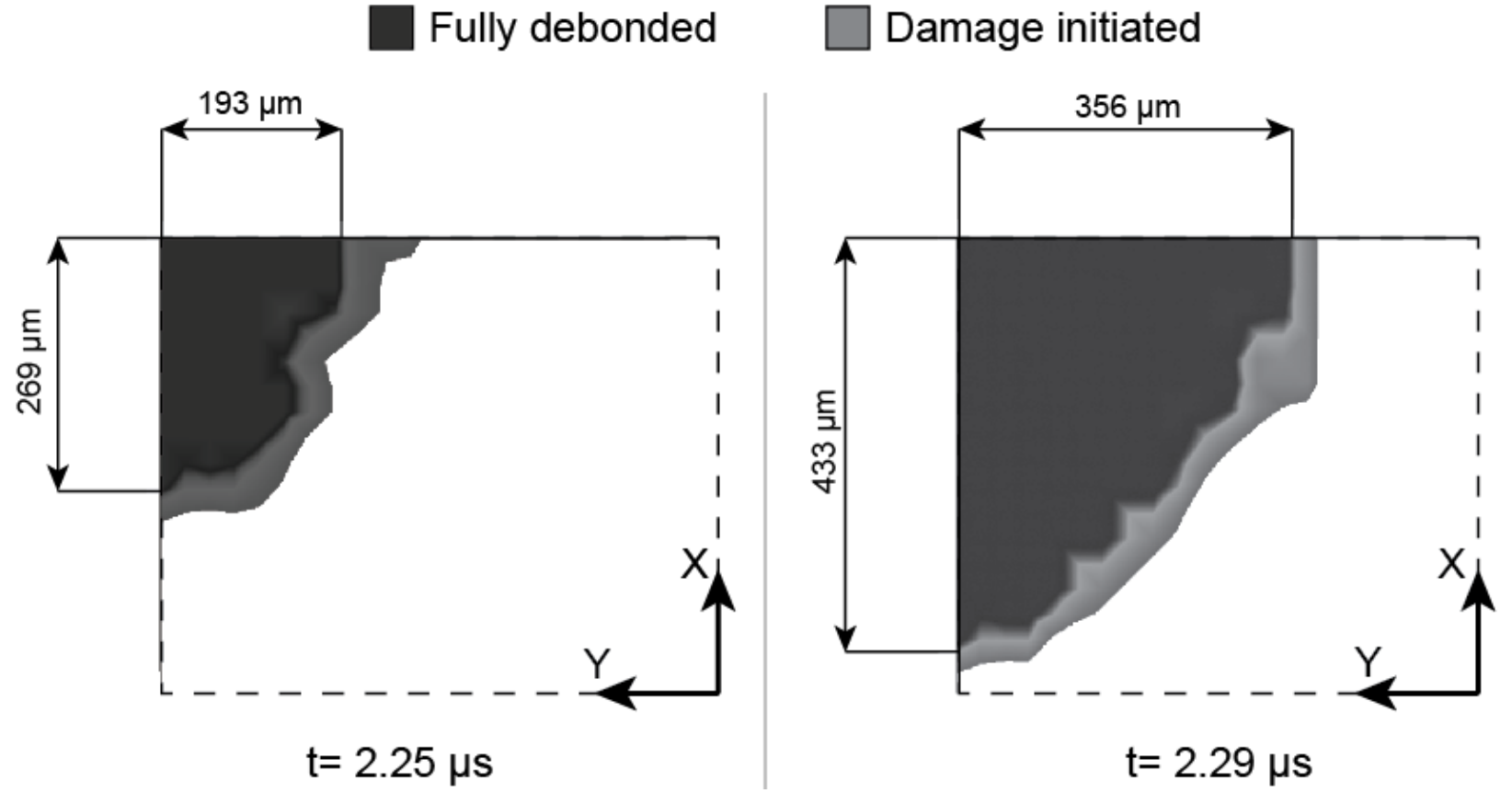

Fig. 3.16 Magnified portion of delamination corresponding to squares in Fig. 3.15 for time steps $\mathrm{t}=2.25 \mu \mathrm{s}$ and $\mathrm{t}=2.29 \mu \mathrm{s}$. 


\subsection{Limitations}

The FEM model focused on crack initiation loading and did not account for the hydrodynamic pressurization associated with delamination when AWJM [45]. Thus, the model was unable to predict the full extent of delamination when AWJ piercing laminate substrates.

A larger $762 \mu \mathrm{m}$ mixing tube was used and analyzed in the initial study, however, the extent of delamination exceeded that of the $20 \mathrm{~mm}$ x $20 \mathrm{~mm}$ dimensions of the specimen (Fig. 3.17). These specimens were removed from the dataset since the full extent of delamination could not be quantified, thus skewing the results.

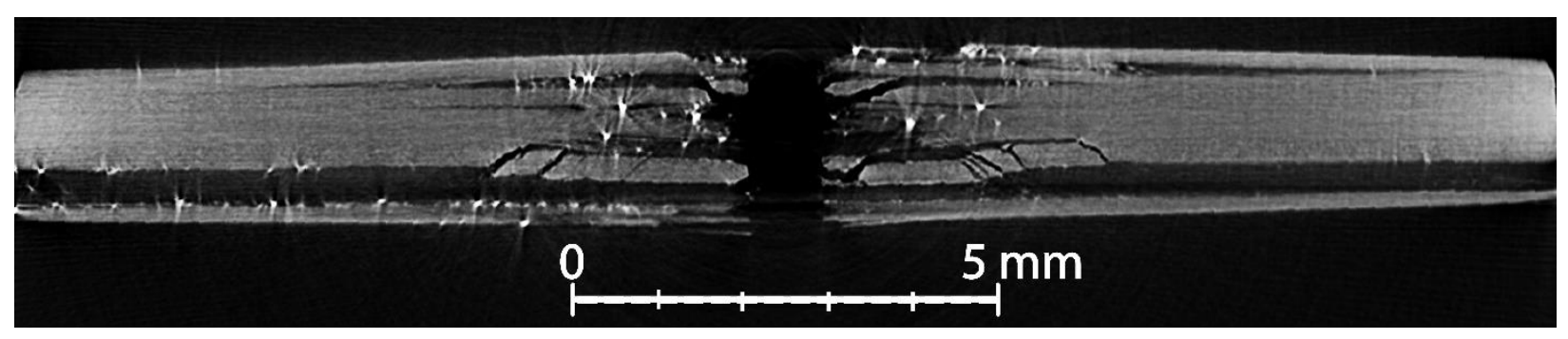

Fig. 3.17 Delamination from a larger $762 \mu \mathrm{m}$ mixing tube nozzle that exceeded the observable space from the micro CT-scans.

Although it is widely assumed that the water-jet front is largely devoid of abrasive particles, this was not verified in the present experiments. Droplet formation from ablated water at the front was also not modelled, but this was unlikely to have caused significant error since the shock pressures were created by the central core of the impacting jet. Similarly, energy equations were not used in FLUENT and thus the thermal effects associated with the compressed water during the impact simulations were not modelled. Consequently, the model did not consider the effect of cavitation during the impact process [162], but this has been shown to be acceptable for hydraulic shock simulations $[110,114]$. Liquid droplet impact differs from continuous jet impact in that the former does not create a stagnation pressure, and cavitation is central to the generation of the loading in droplet impact. The effect of strain rate on the material properties was not considered in the current analysis and this affected the assumed acoustic velocity of wave propagation in the target substrates [120]. 


\subsection{Summary}

A transient, two-way fluid-structure interaction (FSI) model was used to predict delamination of the top ply during the AWJ piercing process in a C-fiber epoxy laminate across a range of pressures and nozzle sizes. The structural component of the FSI model utilized a cohesive zone model to predict delamination onset and propagation. The model results showed that increased AWJ pressure and nozzle size generated larger hydraulic shock loadings as the jet struck the target, and that this increased crack initiation and debonding. The model captured the interdependent relation between the anisotropic properties of the of C-fiber laminate, the distribution of the hydraulic shock pressure on the target surface, and the resulting anisotropic deformation of the surface. The predicted delamination in the top ply of the composite damage was verified experimentally using the x-ray microtomography of the pierced specimens. In Chapter 4, the characterization and modelling of delamination during AWJM will be extended to cutting. 


\section{Chapter 4 Characterizing and modelling delamination of carbon-fiber epoxy laminates during abrasive waterjet cutting}

This chapter is based on the following paper submitted for publication:

J. Schwartzentruber, J.K. Spelt, M. Papini. "Characterizing and modelling delamination of carbon-fiber epoxy laminates during abrasive waterjet cutting", submitted to Composites Part A: Applied Science and Manufacturing

\subsection{Introduction}

AWJM uses an ultra-high pressure water jet to entrain a mixture of abrasive particles and air. AWJM is ideal for cutting and drilling composite materials containing hard reinforcing particles or fibers because it produces no heat affected zone, minimal tool wear, and results in negligible residual stresses and workpiece reaction forces [3]. However, the AWJM of composite materials is prone to fiber pull-out/roll-out [29], matrix cracking [166] and delamination [44].

Nevertheless, Saleem et al. [167], in a comparison of AWJM hole trepanning to conventional hole drilling in composite materials, showed that AWJM led to better fatigue strength characteristics. Delamination is a common problem in both the cutting and drilling of composite laminates because it can reduce the strength and durability of the laminate [128]. Ho-Cheng [44] and Shanmugam et al. [45] described the delamination as two events: the first is the interlaminar separation caused by the thrust of the jet, and the second is the propagation of delamination damage by hydrodynamic pressurization of the interlaminar crack. Ho-Cheng [44] described the delamination during drilling by waterjet piercing using a fracture mechanics approach with plate theory. He proposed a model that relates delamination damage to waterjet pressure and laminate properties. Ho-Cheng [44] observed that delamination occurs near the exit of a through pierce as a result of the reducing strength of the uncut thickness as drilling depth increases. As a result of the reduced strength, increased bending of the plies occurs causing delamination before the laminate is completely pierced. As an interlaminar crack is made, water enters the opening creating a hydrodynamic pressurization. The piercing delamination model of ref. [44] is not directly applicable to cutting because of the different forces generated by the jet on the machining front during piercing compared to cutting. 
Shanmugam et al. [45] explored the mechanisms of delamination when AWJ cutting graphite/epoxy composites and generated a semi-empirical model for predicting delamination. Using a microscope for visual inspection, they observed abrasive particles in cracks and voids adjacent to the cut and concluded that the predominant delamination mechanism in waterjet cutting is the water wedging effect of hydrodynamic infiltration. Their empirical model showed that increasing traverse speed resulted in increased crack length, which was attributed to the decreased abrasive dose, resulting in a decreased cutting efficiency. Wong et al. [83] and Dhanawade and Kumar [168] conducted parametric studies on the delamination of carbon-fiber epoxy composites. Their results showed that delamination was influenced by AFR, traverse speed, and pressure, and that delamination could be minimized by increasing the kinetic energy of the AWJ while reducing the cutting speed. The conclusions drawn from such studies are limited because of possible inaccuracies in measured interlaminar crack lengths using optical/electronic microscopes to examine cross-sections that were cut perpendicular to the cut edges.

Colligan et al. [169] determined that there exists a critical traverse speed and abrasive mass flow rate for a given set of machining parameters that promotes ply separation. This is in agreement with Wang [2] who showed that when the jet is unable to cut through the material, the upper geometry of the kerf resembles that of a through-cut, however, the bottom of the channel forms a pocket. The pocket is attributed to reduced jet effectiveness and stability, causing the jet to deflect sideways and promoting delamination. Hashish [42] performed multiple experiments on cutting, turning, milling and drilling composites and determined that delamination could be eliminated by reducing the jet diameter and supporting the material at the bottom.

In summary, the majority of existing studies have been focused on the morphological characteristics of delamination, and have inferred mechanisms from the visual classification of the extent of delamination. Existing models of delamination have been mostly empirical. This study characterized the extent of delamination during AWJ cutting of carbon-fiber composites using water uptake measurements, and then developed a numerical model of the delamination process to provide insight into the mechanisms responsible for crack propagation. 


\subsection{Experiments}

A moisture uptake experiment was conducted to quantify the extent of delamination under various operating conditions. To differentiate the water-jet loads on the side-wall of the cut from those on the cutting front, an experiment compared the force of the jet during through-cutting and edge trimming. This was then used to identify the location and relative magnitude of the maximum waterjet loading on the composite sheet. Three cutting kerf geometries were measured and then modeled in the FEA, and the predicted delamination was compared with the moisture uptake results.

The composite used in the experiments was a continuous carbon fiber (60\% by wt.)/epoxy, uni-directional ply, laminate $[0 / 90 / 0]_{\mathrm{s}}$ - referred to as CF-epoxy. The material properties of the composite substrate shown in Table 4.1 were provided by the manufacturer. The mode I $\left(G_{I c}=\right.$ $\left.137 \mathrm{~J} / \mathrm{m}^{2}\right)$ and mode II $\left(G_{I I C}=502 \mathrm{~J} / \mathrm{m}^{2}\right)$ were approximated using the same methodology as discussed in Section 3.2.1. The FEM results were relatively insensitive to changes in $G_{I c}$; e.g. increasing $G_{I C}$ by $10 \%$ yielded less than $1 \%$ change in the delamination area predicted by the FEM. The fiber orientation angles shown in Fig. 4.1 correspond to the alignment of the $y$-axis with respect to the jet traversing direction.

\subsubsection{Moisture Uptake Experiment}

AWJ cuts were performed on the composite laminate substrate to assess the extent of delamination (Fig. 4.1). 


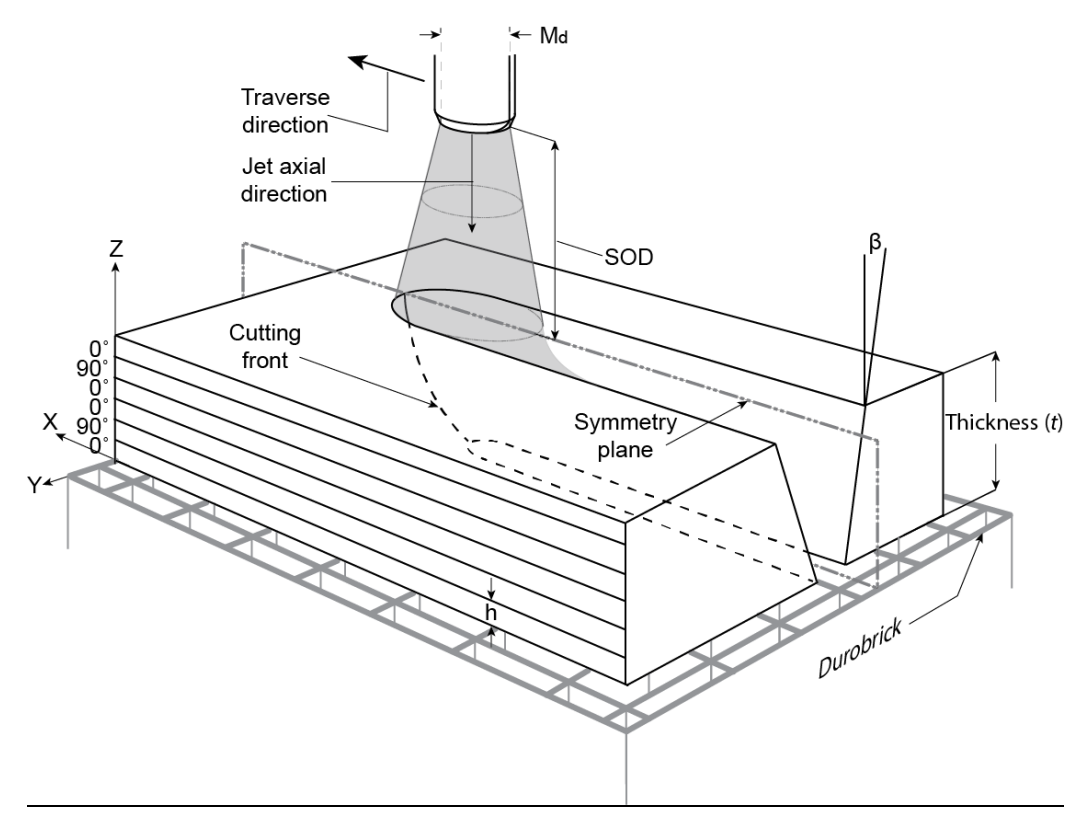

Fig. 4.1 Schematic of AWJ cutting of the carbon fiber epoxy laminate (not to scale) showing sidewall angle $\beta$. Jet traverse shown parallel to $0^{\circ}$ fibers of the first ply. The CF-epoxy sheet from which the specimens were cut fully supported on the bottom and clamped to the AWJ bed restricting lateral and vertical movement. Final specimen sizes were $85 \times 12.5 \mathrm{~mm} . M_{d}$ is the inner mixing tube diameter. The composite sheet $(30 \times 30 \mathrm{~cm})$ was fixed to the cutting bed along its four edges.

Table 4.1 Carbon-fiber/epoxy laminate material properties.

\begin{tabular}{ll}
\hline \multicolumn{1}{c}{ Property } & \multicolumn{1}{c}{ Value } \\
\hline Matrix & $\begin{array}{l}\text { Epoxy } \\
\text { Continuous carbon } \\
\text { Reinforcement }\end{array}$ \\
fiber \\
Fiber weight $\%$ & 60 \\
Fiber orientation & Uni-directional \\
$t(\mathrm{~mm})$ & $1.2 \pm 0.017$ \\
Specific Gravity & 1.53 (ASTM D792) \\
Tensile Strength $(\mathrm{MPa})$ & 1277 (ASTM D3039) \\
Tensile Modulus $(\mathrm{MPa})$ & $122($ ASTM D3039) \\
$E_{11}(\mathrm{GPa})$ & 117 \\
$E_{22}=E_{33}(\mathrm{GPa})$ & 8 \\
$G_{12}=G_{13}(\mathrm{GPa})$ & 5.1 \\
$G_{23}(\mathrm{GPa})$ & 3.74 \\
$v_{12}=v_{13}$ & 0.31 \\
$v_{23}$ & 0.44 \\
$G_{I c}=G_{c, n}\left(\mathrm{~J} / \mathrm{m}^{2}\right)$ & 137 \\
$G_{I I c}=G_{c, t}\left(\mathrm{~J} / \mathrm{m}^{2}\right)$ & 502 \\
\hline
\end{tabular}


The moisture uptake experiments were based on a 6 factor, 3 level Taguchi design of experiments (consisting of 27 tests, with zero replicates) as shown in Table 4.2. The factors used in the study were selected from a consensus in the literature regarding the AWJ process parameters that have been shown to influence crack formation in laminated composites. Pressure, traverse speed, and AFR have been shown by Shanmugam et al. [45], Ho-Cheng [44], and Ramulu and Arola [62] to affect delamination and kerf morphology. Hashish [42] has noted the effect of mixing tube size on delamination. Ho-Cheng [44] and Ramulu and Arola [170] have observed the effect of fiber-orientation when piercing and cutting polymer composites. The levels for each factor were based on previous experiments and ensured that the AWJ had adequate entrainment and cutting power to produce repeatable degrees of delamination damage as assessed using moisture uptake samples. The variation between the levels provided sufficient differences to generate meaningful results.

Table 4.2 Factors and levels used in the moisture uptake tests, the x-ray micro-tomography experiments at the two traverse speeds (medium and fast), and the conditions used in the FE models of the kerf for the slow, medium and fast traverse speeds for each nozzle size.

\begin{tabular}{|c|c|c|c|c|}
\hline Factor & $\begin{array}{l}\text { Moisture } \\
\text { uptake tests } \\
\text { levels }(1,2,3)\end{array}$ & $\begin{array}{l}\text { x-ray micro- } \\
\text { tomography } \\
\text { tests } \\
\text { (medium, fast) }\end{array}$ & $\begin{array}{l}\text { FE kerf models } \\
\text { (traverse } \\
\text { speeds: slow, } \\
\text { medium, fast) }\end{array}$ & $\begin{array}{l}\text { FE kerf models } \\
\text { (nozzle sizes: } \\
254,381,762 \\
\mu \mathrm{m})\end{array}$ \\
\hline Pressure $[\mathrm{MPa}]$ & $225,250,275$ & 250,250 & $150,150,150$ & $150,150,150$ \\
\hline $\begin{array}{l}\text { Stand-off distance } \\
\text { (SOD) }[\mathrm{mm}]\end{array}$ & $1,2.5,5$ & 2,2 & $2,2,2$ & $2,2,2$ \\
\hline $\begin{array}{l}\text { Abrasive flow-rate } \\
\text { (AFR) }[\mathrm{g} / \mathrm{s}]\end{array}$ & $0.2,0.4,0.8$ & $0.2,0.2$ & $3.3,3.3,3.3$ & $3.3,3.3,3.3$ \\
\hline $\begin{array}{l}\text { Mixing tube dia. } \\
\left(\mathrm{M}_{\mathrm{d}}\right) \text { (orifice dia.) } \\
{[\mu \mathrm{m}]}\end{array}$ & $\begin{array}{l}254(128), 381 \\
(178), 762 \\
(356)\end{array}$ & $\begin{array}{l}254(128), 254 \\
(128)\end{array}$ & $\begin{array}{l}254(128), 254 \\
(128), 254(128)\end{array}$ & $\begin{array}{l}254(128), 381 \\
(254), 762(356)\end{array}$ \\
\hline $\begin{array}{l}\text { Traverse speeds } \\
{[\mathrm{mm} / \mathrm{min}]}\end{array}$ & $100,500,1000$ & 1000,3000 & $\begin{array}{l}\text { Zero-taper, 1500, } \\
4000\end{array}$ & 1500 \\
\hline $\begin{array}{l}\text { Top ply fiber } \\
\text { orientation }\left[{ }^{\circ}\right]\end{array}$ & $0,45,90$ & 0 & 0 & 0 \\
\hline
\end{tabular}

An OMAX 2626 Jet Machining Center (OMAX Corp. Kent, WA, USA), fitted with programmable nozzle movement allowing positional accuracy of $\pm 80 \mu \mathrm{m}$ over a $30 \mathrm{~cm}$ length, was used to perform the experiments. All experiments were conducted unsubmerged; i.e. with 
the jet exposed to air. The jet was allowed to reach equilibrium before cutting to avoid impact delamination damage. The abrasive used in the experiments was $75 \mu \mathrm{m}$ garnet (Barton HPX ${ }^{\circledR}$, Glen Falls, NY, USA) with a Mohs hardness of 7.5-8.5, and a density of $4000 \mathrm{~kg} / \mathrm{m}^{3}$ [66]. Fig. 2.1 shows the distribution of the abrasive particle projected area diameters, based on sieve analysis data provided by the supplier.

Based on preliminary cutting experiments (Section 4.4), it was observed that the maximum delamination damage under the extreme conditions of Table 4.2 was approximately $5 \mathrm{~mm}$ in length based on visual inspection using a scanning electron microscope. The moisture uptake samples were cut into $12.5 \mathrm{~mm} \times 100 \mathrm{~mm}$ rectangular pieces from a $0.75 \times 0.5 \mathrm{~m}$ CF-epoxy sheet using the AWJ. These dimensions maximized kerf surface area for the moisture uptake analysis (which had a maximum length of $100 \mathrm{~mm}$ due to apparatus limitations). The samples were clamped along their edges to the top of an OMAX Durobrick (OMAX Corp., Kent, WA, USA) during cutting. The flat Durobrick was made from a perforated polypropylene which allowed the jet to pass through it without creating a secondary slurry flow, but yet provided sufficient support to minimize deflections of the thin composite plate during cutting.

In Fig. 4.1, the traverse direction is shown parallel to the $x$-axis. To assess other cutting directions, the composite substrate was rotated about the $z$-axis by $45^{\circ}$ and $90^{\circ}$, thus mimicking the jet cutting through a $\left[45^{\circ}, 45^{\circ}, 45^{\circ}\right]_{\mathrm{s}}$ and $\left[90^{\circ}, 0^{\circ}, 90^{\circ}\right]_{\mathrm{s}}$ layup, respectively. To eliminate the added local damage the jet can create when traversing onto and then off the end of the specimen [171], approximately 7.5. $\mathrm{mm}$ was cut from each end using a slow speed diamond saw, giving a final specimen length of $85 \mathrm{~mm}$. One specimen was used for each cutting condition.

Previous studies quantifying moisture absorption of composite materials are commonly associated with electrochemical impedance spectroscopy (EIS) [172-175]. Water absorption increases the conductivity and capacitance of the composite which can be quantified by the impedance of the structure. This method is commonly used to assess bond strength degradation over extended periods of time $[172,173]$. EIS is more suitable for quantifying moisture absorption through Fickian diffusion [176-178] of the epoxy matrix (carbon fibers do not absorb water [179]), rather than the relatively simplistic quantification of voids generated by delamination. Thus, an alternate method quantifying water uptake was developed to assess the damage generation. 
The extent of delamination during the AWJ cutting process was determined by measuring the mass of water infiltration into the surface micro-cracks of the composite substrate. The moisture uptake analysis was performed using an HR83 moisture analyzer (Mettler-Toledo, Columbus, Ohio, USA). Before submerging the samples, the specimens were baked at $105{ }^{\circ} \mathrm{C}$ for 10 min to remove any moisture absorbed during the machining process or from the ambient air. The specimens were then submerged in distilled boiling water for $45 \mathrm{~min}$ to ensure adequate penetration of the water into edge cracks. The thermosetting epoxy and carbon fibers were unaffected by the baking and submerging process. Once removed from the water, the exterior of the samples were superficially dried using a micro-fiber cloth and placed into the moisture analyzer. The moisture analyzer acted as a high precision scale and oven, evaporating the water contained in the specimens while recording the percentage change in moisture content. The samples were assumed to be at steady state when there was no change in the percentage of moisture content, beyond a minimum recording time of $10 \mathrm{~min}$.

\subsubsection{Measurement of Jet Loading Force}

The relative magnitudes of the forces of the jet on the cutting front and kerf sidewall were inferred by performing through-cuts and edge trims, respectively, on specimens fitted with a strain gauge (Fig. 4.2). Since the objective was only to obtain the relative magnitudes on the front and kerf sidewall, a stainless steel target was used instead of the CF-epoxy because of its isotropic properties, which made it easier to infer the load from the strain gauge readings. Previous comparisons amongst different ductile materials cut using AWJ [58, 180-183] suggested that the relative differences in cutting front and taper geometry between stainless steel and CF-epoxies would be small.

The linear pattern strain gauge $(3.18 \mathrm{~mm} \times 1.78 \mathrm{~mm}, 350 \mathrm{ohm} \pm 0.6 \%$, Micro Measurements, Raleigh, NC, USA) was bonded to a 304 stainless steel ( $E=200 \mathrm{GPa}$ [184]) sacrificial plate, and connected to a Cronos-PL2 (IMC Gmbh, Berlin, DE) data acquisition system, recording at 10 $\mathrm{kHz}$. Through and trim cuts were made at a pressure of $250 \mathrm{MPa}, 3 \mathrm{~mm} \mathrm{SOD}, 100 \mathrm{~mm} / \mathrm{min}$ traverse speed, $0.040 \mathrm{~kg} / \mathrm{min}$ AFR, using a nozzle with a $254 \mu \mathrm{m}$ mixing tube diameter, and 128 $\mu \mathrm{m}$ orifice diameter. The trimming offset (Fig. 4.2) was $50 \mu \mathrm{m}$. The through cuts represented the 
loading conditions produced by the waterjet, inclusive of the cutting front and kerf taper loadings. The trimming cuts revealed mostly the loading force on the side wall since the cutting front was negligibly small in this case.

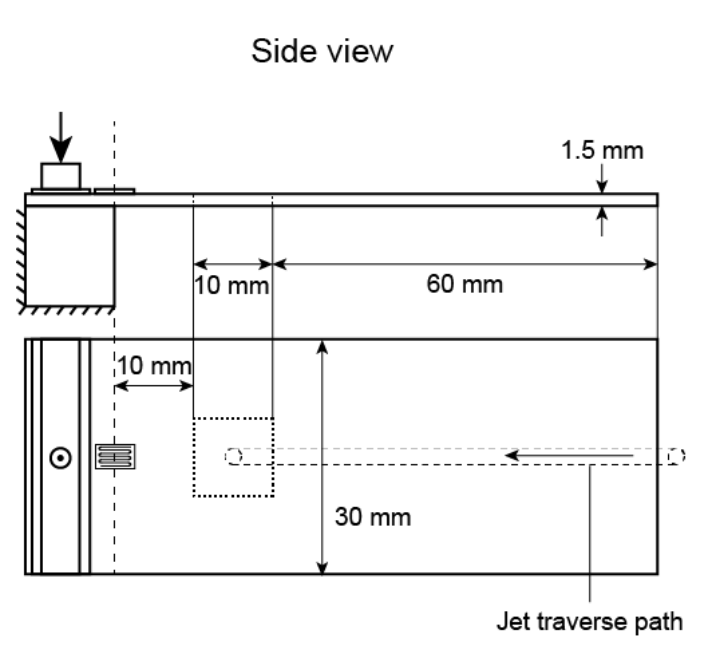

Top view

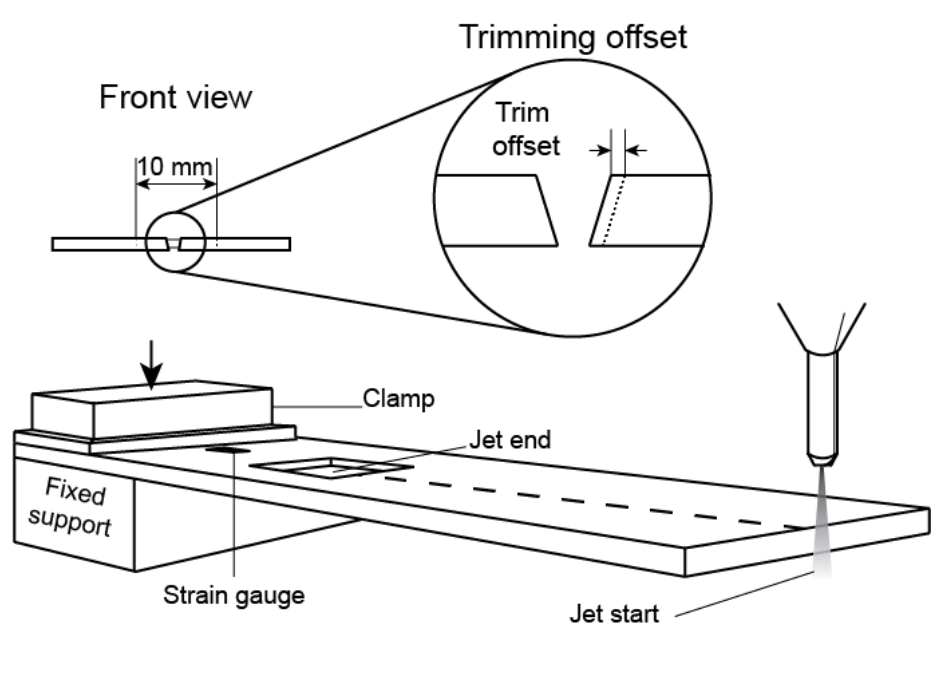

Isometric view

Fig. 4.2 Schematic of the force transducer used to measure the loads applied to the cutting front and kerf edges by the water jet.

\subsubsection{Micro-tomography and Characterization of the AWJ Cut Geometry}

Kerf taper [2, 35, 37, 61, 62] and cutting front curvature (i.e., jet lag) [13, 58, 180, 181, 185] have been found to increase with operating conditions that tend to decrease the erosive efficacy. For example, a high traverse speed and low AFR lead to a larger kerf sidewall taper [84], a higher cutting front curvature [38, 186], a lower cut quality $[2,61]$ [187] and ultimately more delamination $[166,188]$. These trends continue until the jet has insufficient cutting power to penetrate the material and a milled channel is formed. Thus, three case studies at slow (Fig. 4.5a), medium (Fig. 4.5b) and fast (Fig. 4.5c) traverse speeds were generated to represent the ranges of cut quality in AWJM. The case studies produced varying degrees of taper and cutting front curvature for use in the FE analyses of Section 4.3. The fast traverse case produced a milled 
channel that penetrated to half the thickness of the bottom ply (the channel depth was $92 \%$ of the total sheet thickness), while the medium traverse case was machined as a through-cut with a slight taper and reduced cutting front curvature. Although the fast case was a channel, because of its depth, it was taken to represent the transition kerf geometry at the point where a through-cut was first formed. The operating conditions used to produce the medium and fast cases are shown in Table 4.2. The slow case was not machined but modeled as an idealized AWJ cut with zero taper and zero cutting front curvature. Therefore, it represented the kerf geometry at the opposite extreme of the fast case.

To visualize the cutting front morphology and amount of delamination produced by AWJ cutting, 3D x-ray micro-tomography (micro-CT) was used. In addition to the medium and fast cases described above, additional cuts were assessed using traverse speeds from $500 \mathrm{~mm} / \mathrm{min}$ to $5000 \mathrm{~mm} / \mathrm{min}$, in $500 \mathrm{~mm} / \mathrm{min}$ increments, with the remaining operating conditions held constant at the values shown in Table 4.2. The x-ray micro-tomography scans were performed using a Skyscan 1172 (Bruker Corp., Billerica, MA, USA), with a $20 \mathrm{~mm}$ maximum object size and 0.5 $\mu \mathrm{m}$ resolution. The resulting scan data was analyzed using NRecon (Version 1.6.5.0, Bruker Corp., Billerica, MA, USA) and the open-source image analysis software, ImageJ.

Orthogonal slices of the CT scans in the positions and orientations shown in Fig. 4.3 were used to show the delamination surrounding the cutting front.

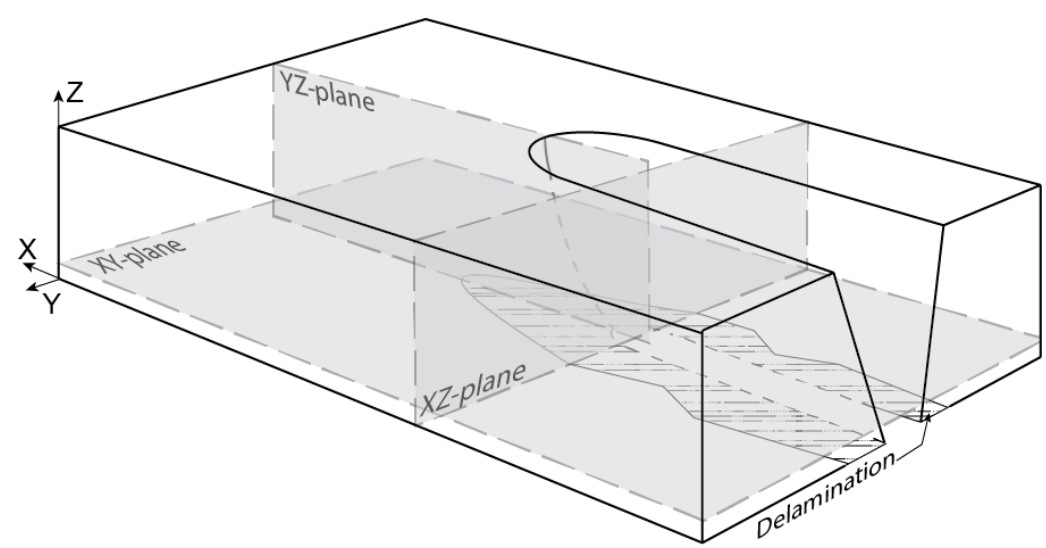

Fig. 4.3 Position and orientation of the orthogonal slices in the CT scans in the vicinity of the cutting front. $X Y$-plane is approximately $200 \mu \mathrm{m}$ from the bottom of the composite sheet. 
To resolve the cutting front shape, the experiment utilized a modification of the method proposed by Zhang et al. [180], who abruptly stopped the abrasive entrainment. The modified procedure was required since the water-only jet that remained after ending the abrasive entrainment was found to be of sufficient erosive energy to modify the cutting front geometry on the present material, which eroded at a higher rate than the aluminum used by Zhang et al. [180]. Therefore, the AWJ was instead traversed over a shielding material ( $1 \mathrm{~mm}$ stainless steel feeler gauge), abruptly stopping the cutting. The operating conditions used to generate the kerf profiles for modelling the medium and fast cutting geometries are shown in Table 4.2. The cutting front (Fig. 4.4a) and taper (Fig. 4.4b) of the two cutting cases were measured using an optical profilometer having a lateral resolution of $426 \mathrm{~nm}$ and depth resolution of $16 \mathrm{~nm}$ (ST400, Nanovea Inc., CA, USA), and normalized by the maximum depth of each feature. To generalize the shape of the cutting front profile, an initial measurement was taken along the centerline of the channel/cut, followed by two subsequent scans offset by $10 \mu \mathrm{m}$ on both sides of the initial scan. The measured trends in Fig. 4.4a agree with the generic curved cutting front found by Hashish [186], Orbanic and Junkar [182], and Deam et al.[183]. In agreement with Fig. 4.4b, other investigators have shown that the kerf taper can be well approximated as a line of constant slope (i.e., at a taper angle) [84, 189, 190].

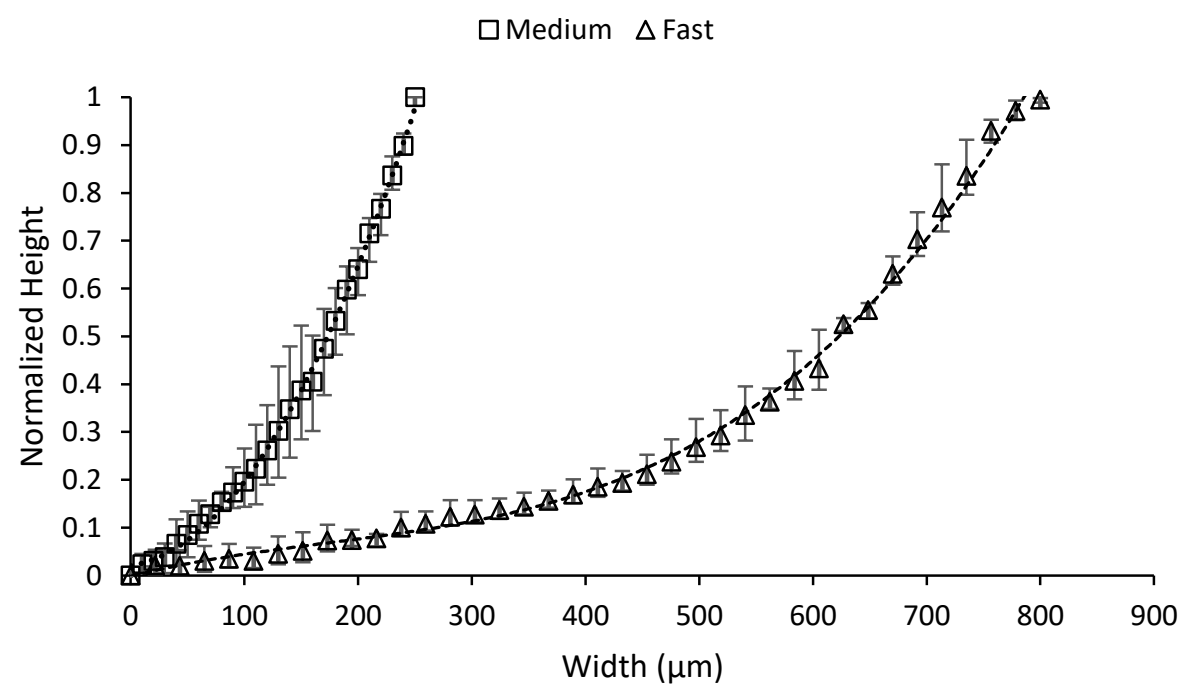

a. 


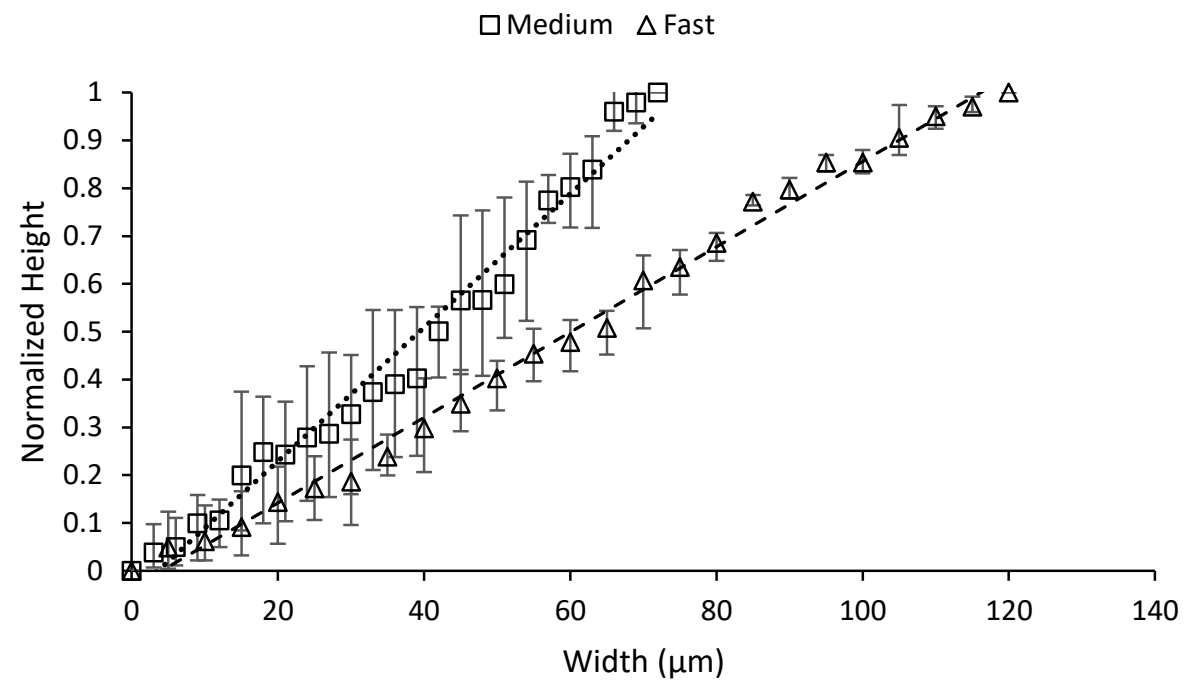

b.

Fig. 4.4 a. Profilometer scan of the cutting front for medium and fast traverse speeds. The best-fit curves are $3^{\text {rd }}$ degree polynomials. b. Profilometer scan of the kerf taper for medium and fast traverse speeds fitted to straight lines. Height measurements were normalized using the maximum value in each case. Error bars represent maximum and minimum values of 3 measurements at different sections on the same specimen.

\subsection{Modelling}

The general approach to modeling the cutting process is analogous to that used in Section 3.3 for piercing of the same material.

\subsubsection{FSI Model Overview}

A FSI analysis was conducted for the slow (zero-taper, Fig. 4.5a), medium (Fig. 4.5b), and fast (Fig. 4.5c) cases shown in Table 4.2, using a 1-way FEM/CFD coupled analysis in ANSYS 15 (ANSYS Inc., Cecil Township, PA, USA). The CFD domain was first solved, and the pressure loads were exported to the structural domain for stress and fracture analyses. The 3D representations of the AWJ cuts for the CF-epoxy substrate (Fig. 4.5) were obtained from the taper and cutting front measurements (Section 4.2.3), that were fitted to a polynomial curve fit 
and imported into ICEM (ANSYS Inc., Cecil Township, PA, USA) and ANSYS Design Modeler (ANSYS Inc., Cecil Township, PA, USA).

a.

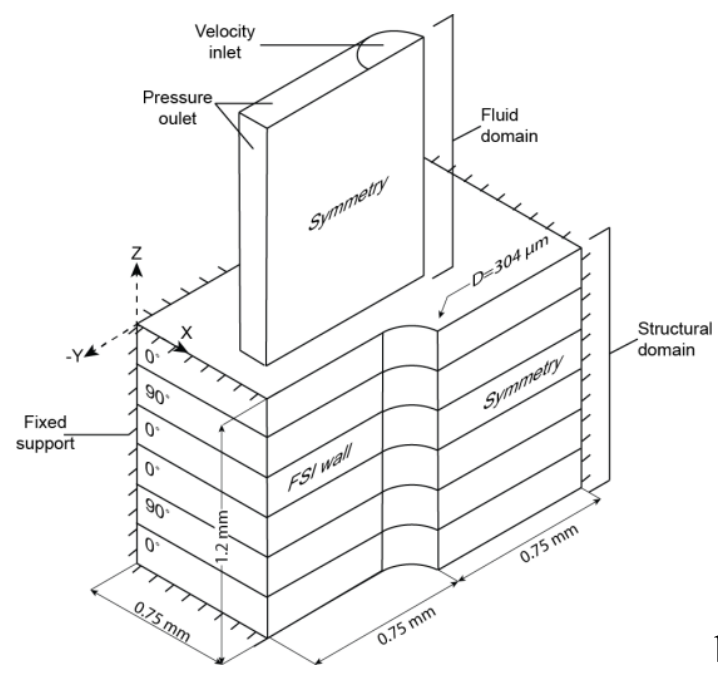

b.

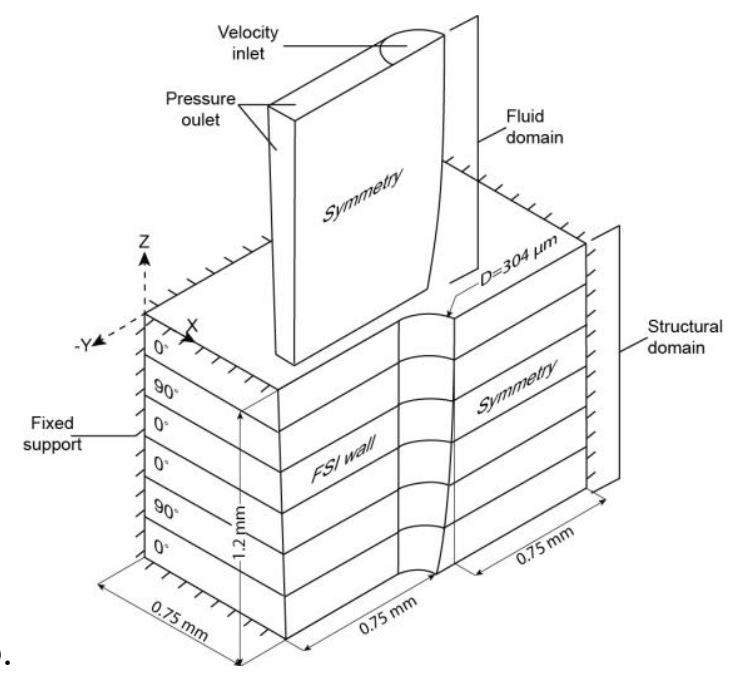

c.

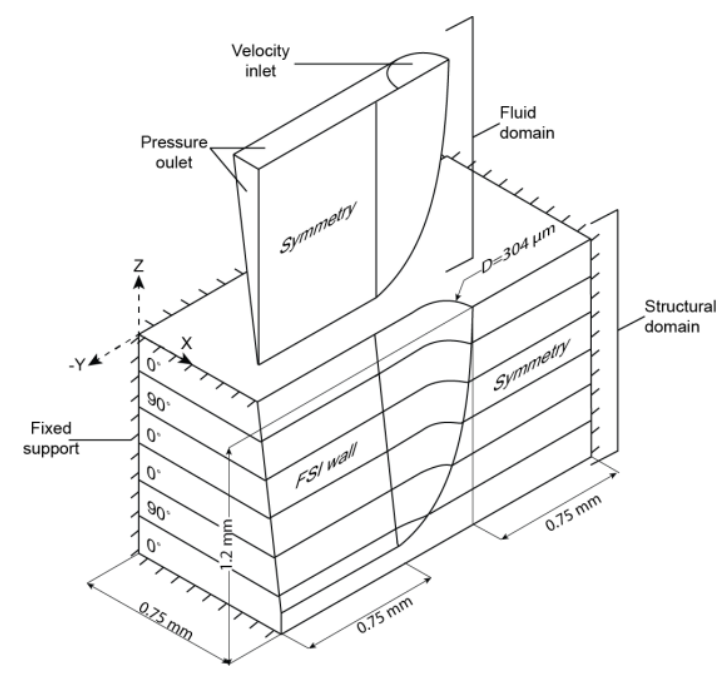

Fig. 4.5 Schematics of the three traverse speed case studies and their control volumes for the CFD/FEM coupled analysis: a. slow traverse speed, b. medium traverse speed, c. fast traverse speed. The footprint diameter of $304 \mu \mathrm{m}$ was for the $254 \mu \mathrm{m}$ nozzle.

The control volume of the FSI simulation was a combination of a CFD domain (Section 4.3.2) and structural domain (Section 4.3.3), as shown in Fig. 4.6. The coupled analysis first solved the waterjet loading exerted on the wall using the CFD domain. The calculated pressure contour was then imported into the structural model and mapped to the shared kerf wall. The structural model replicated the orthotropic properties of each ply (Table 4.1) and bonded the 
plies in their respective layup orientation using CZM elements. The boundaries of the structural control volume were fixed (Fig. 4.5).

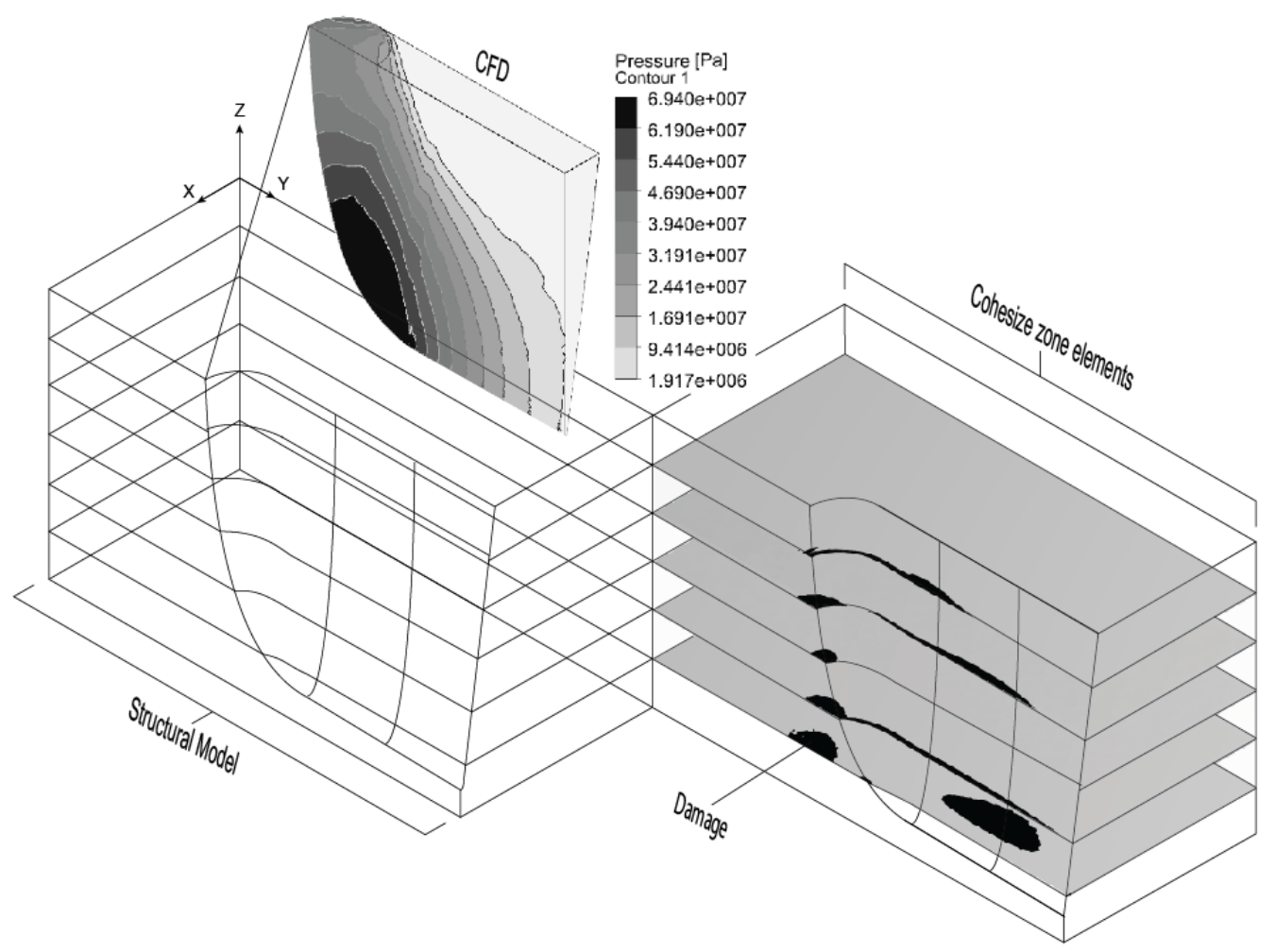

Fig. 4.6 Predicted pressures and CZM inter-ply damage regions on the cut sidewalls from the 1way FSI model for the fast traverse speed.

To maintain consistent reactionary forces between the models, the cutting front geometry was given a $0.75 \mathrm{~mm}$ bounding box from the front tip to the rear of the cutting front in the $\pm y$ direction, and $0.75 \mathrm{~mm}$ from the bottom of the kerf in the positive $x$-direction. As shown in Fig. 4.5, the coupled FSI model required the generation of two control volumes: a fluid domain CFD (Section 4.3.2), and a structural FE domain (Section 4.3.3). 


\subsubsection{CFD Model}

The waterjet flow domains were solved by ANSYS Fluent 15.0 (Ansys Inc., Cecil Township, PA, USA). A symmetric, multiphase, steady, VOF model was used for the waterjet surrounded by air. A $k-\varepsilon$ shear-stress transport turbulence model was used with 170000, 73000, and 94000 quad/tri elements, for the fast, medium and slow cases, respectively, with a convergence residual of $10^{-4}$. The fluid domain did not consider the discrete abrasive particles since the majority of the loading during AWJ cutting is due to the water; i.e., the mass fraction of water 15-140 times that of the abrasive for the present operating conditions [71]. The waterjet velocities and VOF air-towater ratio of the inlet boundary condition were estimated using the 1-D CFD model developed by Narayanan et al. [71], which has been shown to predict axial particle velocities exiting an AWJ nozzle within 5\%. The fluid radial velocity distribution profiles used at the inlet boundary were implemented in a user-defined sub-routine and assumed to follow a $1 / 7^{\text {th }}$ power law function, based on the work of Wang et al. [126].

The models for the $381 \mu \mathrm{m}$ and $762 \mu \mathrm{m}$ nozzles (Table 4.2) were approximated by scaling the $x$ - $y$ coordinates of the $254 \mu \mathrm{m}$ nozzle kerf geometry for the medium traverse speed case (i.e., Fig. $4.5 \mathrm{~b}$ ) by the ratio of jet spreading (which was assumed to be constant); i.e. the ratio of the jet footprint diameter to the mixing tube diameter. The medium traverse speed case for the $254 \mu \mathrm{m}$ nozzle had a top surface cut width of $304 \mu \mathrm{m}$, resulting in a cut width $(W)$ to mixing tube $\left(D_{m}\right)$ ratio of 1.2. The $z$-direction kerf coordinates were not scaled since the plate thickness was constant in the experiments. The domain dimensions were then adjusted so that the $0.75 \mathrm{~mm}$ distance (Fig. 4.5) around the cutting front geometry was retained, thereby ensuring a consistent sheet stiffness surrounding the kerf region. The CFD inlet velocity was the same in all cases.

\subsubsection{Structural Model}

A symmetric, steady, structural simulation was conducted using ANSYS 15.0 Workbench (Ansys Inc., Cecil Township, PA, USA). The structural volume was meshed adaptively with SOLID186 elements (using reduced integration) with a maximum element size of $25 \mu \mathrm{m}$. A convergence study showed that the results were independent of the element size. The orthotropic 
properties of the laminate plies were modelled based on the manufacturer provided specifications (Table 4.1). The layers were bonded with zero thickness 3D, surface-to-surface, pair-based, contact elements (CONTA174 and TARGE170) with the Ansys "pure penalty formulation" to enable the use of the CZM. Interlaminar crack propagation was predicted by activating CZM for the contact elements using a fracture-based debonding model. The contact elements were subject to interlaminar normal $\left(\sigma_{z}\right)$, and shear stresses $\left(\tau_{x z}\right.$ and $\left.\tau_{y z}\right)$, representing mode I, II and III crack propagation stresses, respectively [128]. Because of the varying geometry of the AWJ cut, the side wall and cutting front experienced both normal and shear tractions. The cohesive elements used a mixed-mode, bi-linear, traction separation law to define debonding as shown in Fig. 3.5, with the normal and tangential cohesive tractions expressed in equations (3.10)- (3.13) and fracture envelope defined in equation (3.14) [147].

The maximum normal $\left(\sigma_{c, n}=45 \mathrm{MPa}\right)$ and tangential $\left(\sigma_{c, t}=60 \mathrm{MPa}\right)$ contact stresses were approximated as an average of those found in the literature involving the CZM of carbon/epoxy laminates [149-158]. It was confirmed that the initial stiffnesses of the interfaces $\left(K_{n}\right.$ and $\left.K_{t}\right)$ had a negligible effect on the load predictions, as was also observed in [159]. Therefore, following Refs. [160] and [161] both were set to $10^{14} \mathrm{~N} / \mathrm{m}^{3}$.

To compare to the moisture uptake results which assessed the total delamination damage due to cutting, the delamination predicted by the model was approximated as the total area of CZM nodes in all plies that had separated. This was obtained by solving the model iteratively in load steps to the full jet load.

\subsubsection{Experimental Results and Discussion}

Fig. 4.7 shows that the delamination crack length of the CF-epoxy substrate as a result of the AWJ cutting was on the order of $5 \mathrm{~mm}$. Thus, the width of the moisture uptake samples (12.5 $\mathrm{mm}$ ) was more than twice the length of the maximum observed crack length, thereby mitigating the possibility of interlaminar cracks bridging from either side of the AWJ cut specimen. 


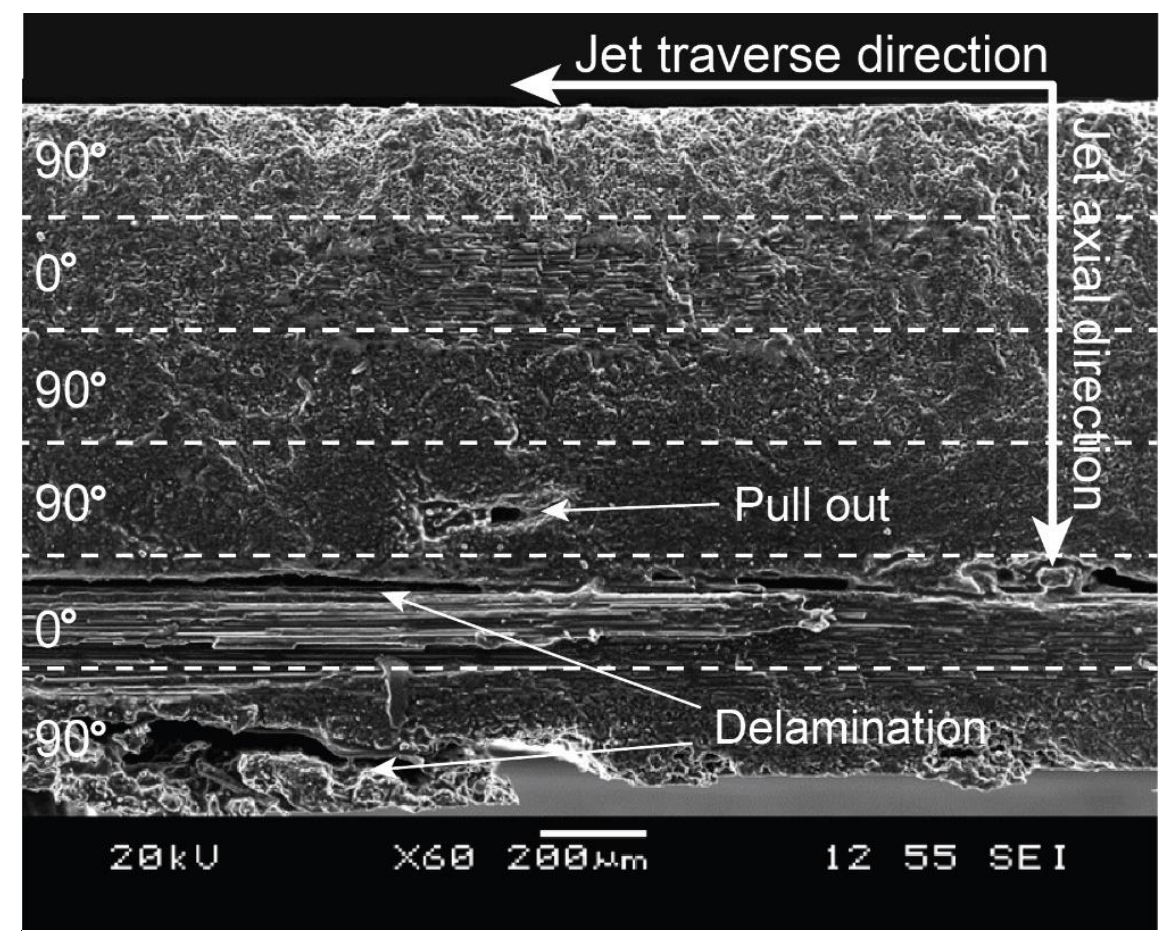

Fig. 4.7 Scanning electron micrograph of delamination in the CF-epoxy substrate as a result of AWJ cutting. Operating conditions: $P=225 \mathrm{MPa}, \mathrm{SOD}=2 \mathrm{~mm}, \mathrm{AFR}=0.93 \mathrm{~g} / \mathrm{s}$, traverse speed: $2000 \mathrm{~mm} / \mathrm{min}, 254 \mu \mathrm{m}$ mixing tube diameter and $128 \mu \mathrm{m}$ orifice diameter. Top ply was oriented with fibers perpendicular to the jet traverse; i.e. at $90^{\circ}$ as shown.

\subsubsection{Comparison of the Jet Loading for Cutting and Trimming}

Fig. 4.8 shows the strain-time recordings comparing the through and trim cuts. The average strain along the length of the through-cut in the 304 stainless steel was $47.2 \mu \varepsilon$, compared to 7.5 $\mu \varepsilon$ for the $50 \mu \mathrm{m}$ trim cut. Therefore, the loading to both kerf side walls produced approximately $15 \mu \varepsilon$ which was $32 \%$ of that for the through-cut, with the remaining $68 \%$ attributable to the cutting front load. This suggested that crack initiation and delamination during AWJ cutting was primarily due to the loading on the cutting front. This is consistent with the FE model predictions of Fig. 4.6, which shows that the large pressure at the bottom of the curved cutting front resulted in a large damage zone in the cohesive elements ahead of the cutting front. This result was confirmed by the micro-CT scans of Fig. 4.9, discussed in the next section, which shows a large crack propagating ahead of the cutting front. It is also consistent with the experimental results of Fig. 4.10 and numerical results of Fig. 4.15, discussed below. Thus, it is shown that increased 
traverse speed (and thus increased cutting front curvature) resulted in increased loading, and thus increased damage.

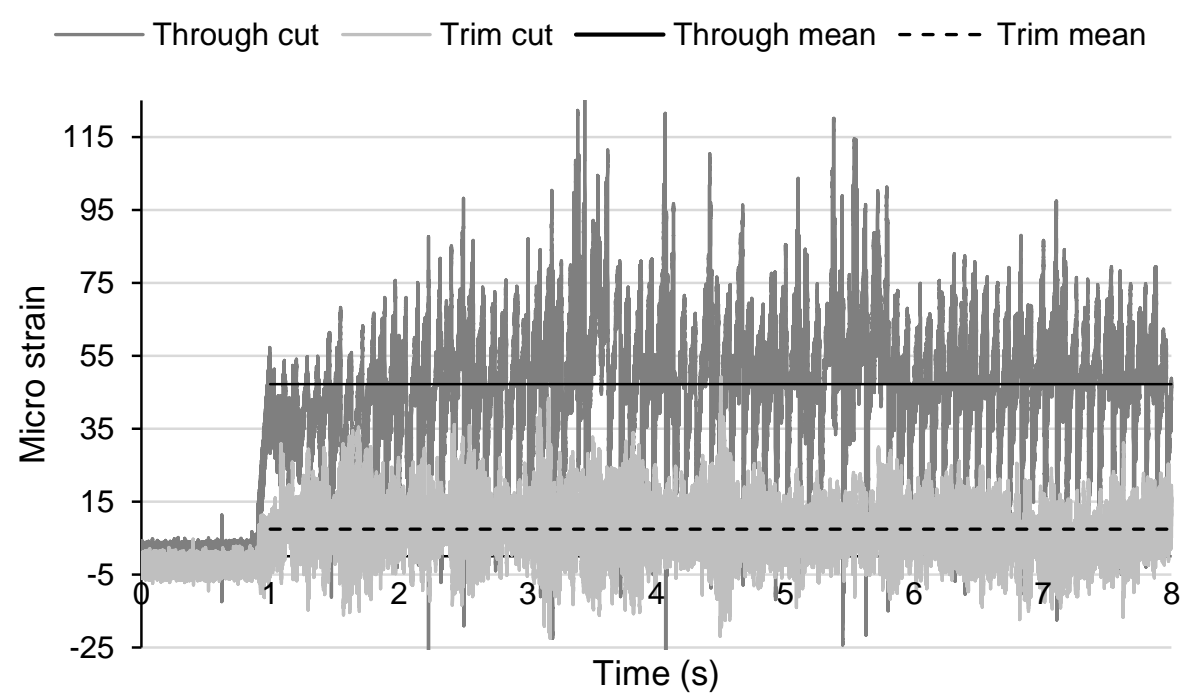

Fig. 4.8 Strain-gauge measurements for the through and trim cut experiments.

\subsubsection{D X-ray Micro-tomography of AWJM Through-Cuts}

Fig. 4.9 shows cross-sections of AWJ cuts in the CF-epoxy. Both the high and medium traverse speed cases showed crack initiation occurring ahead of the cutting front and along the sidewalls of the kerf, after which hydrodynamic pressurization of the initiated micro-cracks extended delamination [44, 45]. Fig. 4.10 shows abrasive grains wedged into an interlaminar crack along the side-wall of the CF-epoxy as a result of re-directed jet flow, thus confirming that hydrodynamic pressurization is contributing to delamination during AWJ cutting. The $x y$-plane in Fig. $4.9 \mathrm{a}$ and b, shows that delamination enveloped the entire cutting front. As cutting continued, the delamination ahead of the cutting front was eroded away (i.e., shaded area in the $x y$-plane of Fig. $4.9 \mathrm{a}$ and b, with the crack propagation in the $y$-direction remaining. The cracks ahead of the cutting front in Fig. 4.9 were measured to be $3.0 \mathrm{~mm}$ and $2.2 \mathrm{~mm}$ for the fast and medium case, respectively. The effect of traverse speed on damage will be discussed in Section 4.5.2.2. 

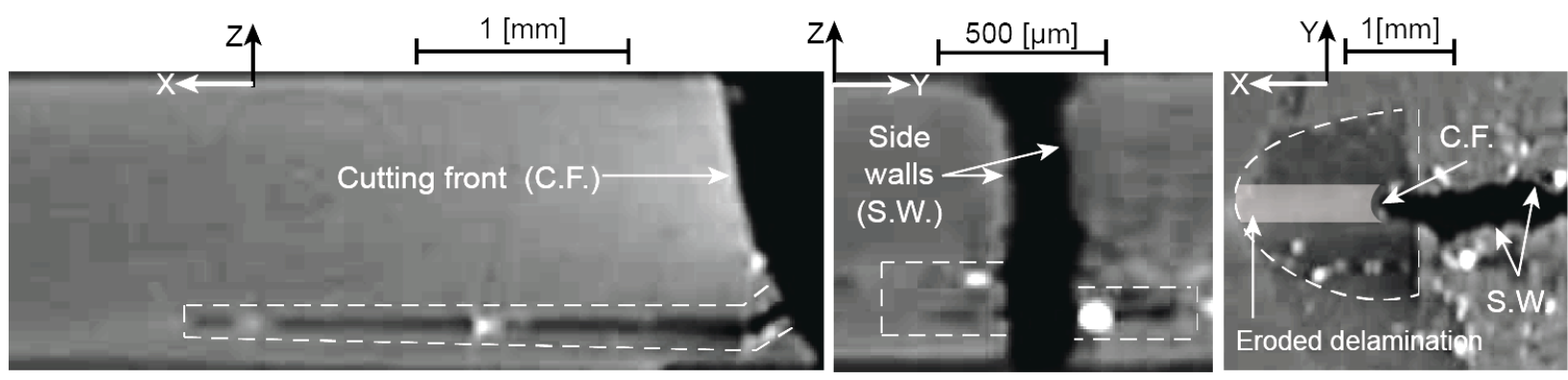

a.

b.
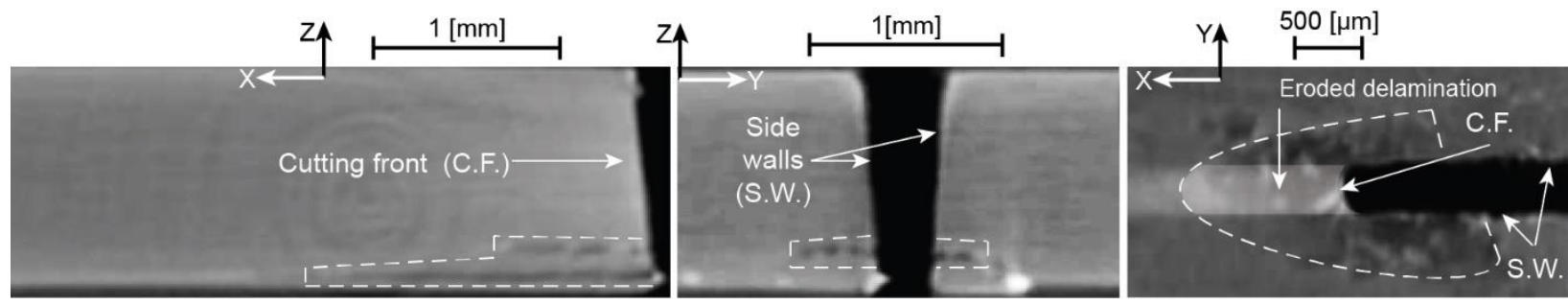

Fig. 4.9 CT scans of AWJ cuts corresponding to the a. fast, and b. medium traverse speeds with operating conditions shown in Table 4.2. Dashed lines encompass delamination damage. Fibers oriented as in Fig. 4.1. The rectangular shaded areas shown in the $x y$-plane indicate the delamination that would be eroded by the advancing cutting front. The depth of the $x y$-planes (right-most micrographs) in the $z$-direction was $\sim 200 \mu \mathrm{m}$.

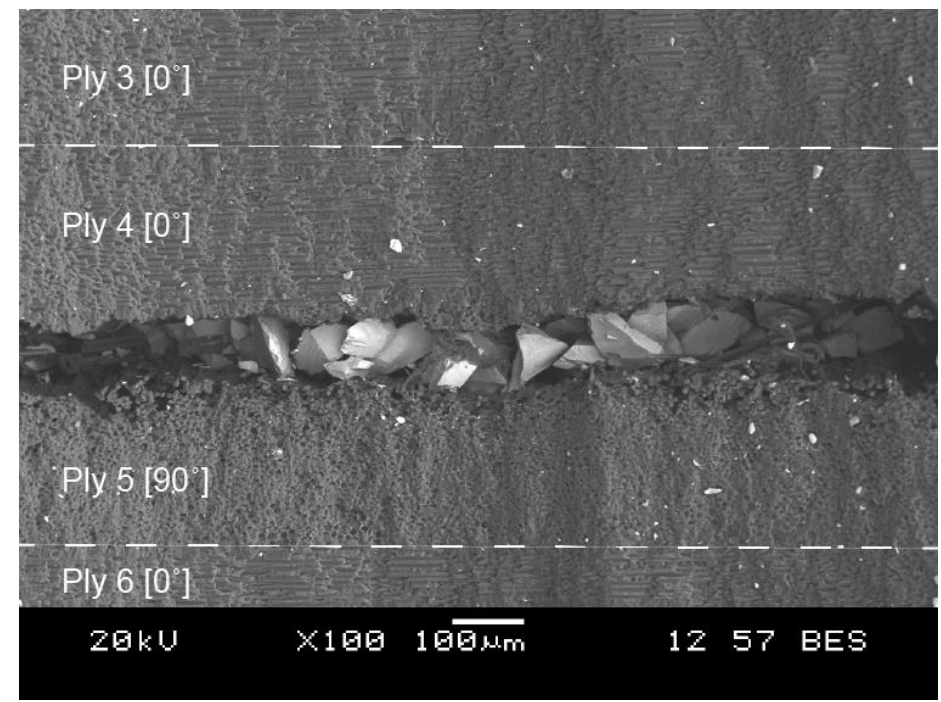

Fig. 4.10 Scanning electron micrograph of abrasive grains (garnet) wedged into an interlaminar crack along the side-wall of an AWJ cut. Operating parameters were: pressure $=225 \mathrm{MPa}$, $\mathrm{AFR}=0.9 \mathrm{~g} / \mathrm{s}$, and a traverse speed $=4000 \mathrm{~mm} / \mathrm{min}$, using the smallest nozzle size. 


\subsubsection{Moisture Uptake Results}

The results of the moisture uptake experiment are shown in Fig. 4.11 as a function of the six selected process parameters (Table 4.2). The number of specimens used in the moisture uptake experiments were limited based on available resources, however, statistical analysis of results provided additional evidence to support the findings of the FSI model, and the X-ray microtomography.

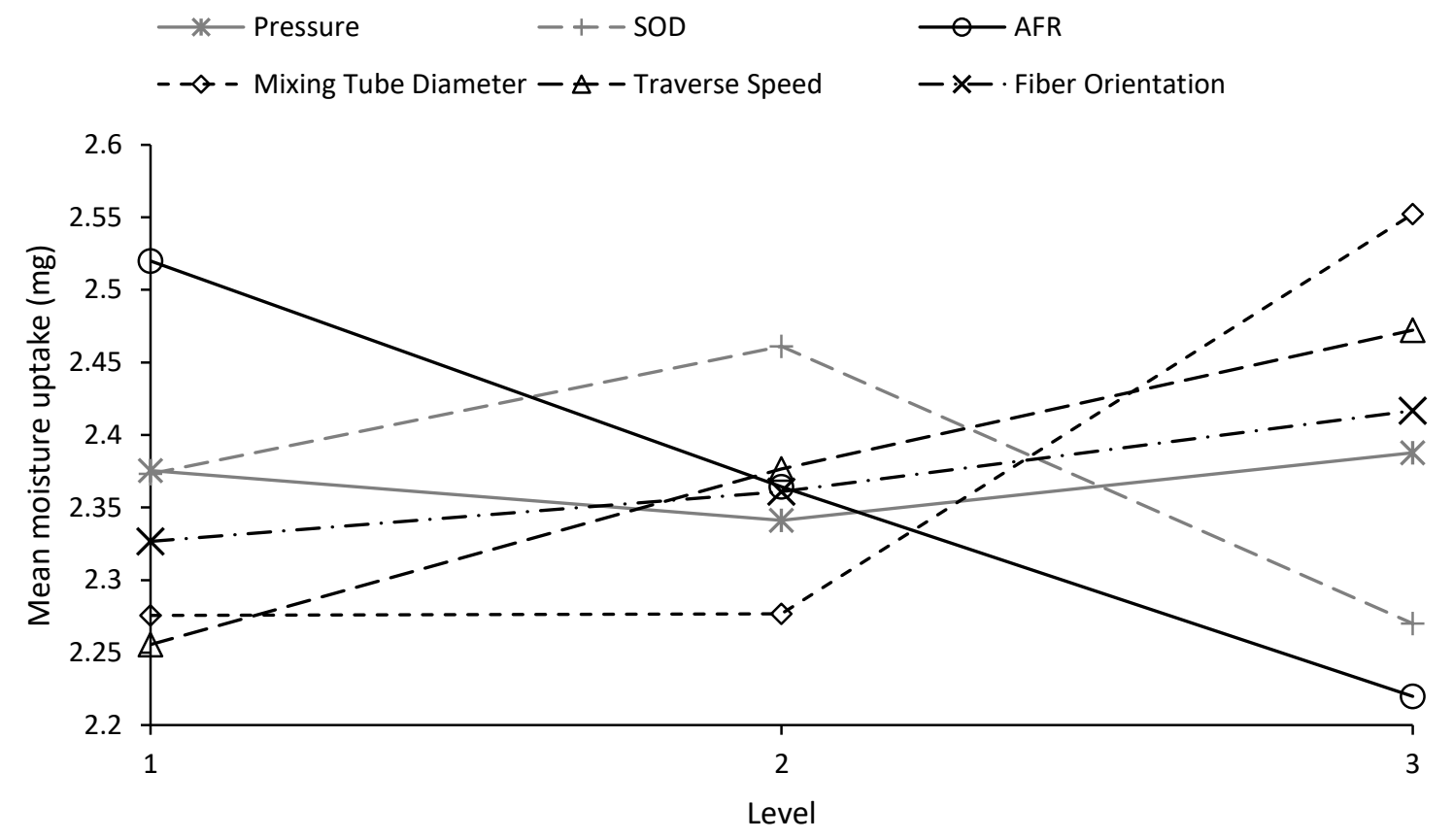

Fig. 4.11 Moisture uptake experimental results.

An analysis of variance (ANOVA) was conducted on the results from the 6-factor, 3level Taguchi design of the moisture uptake experiments using Minitab 17 (Minitab Inc., State College, PA, USA) to determine the significant responses. Prior to analyzing the data, a Box-Cox transformation was applied to improve the normality of the data. As indicated in Table 4.3, the ANOVA computed significant or nearly significant main effects for AFR, traverse speed and nozzle size on the moisture uptake response. 
Table 4.3 Significant ANOVA results. 'Sig.' represents the probability that data could occur by chance.

\begin{tabular}{llll}
\hline Effect & F-Value & Degrees of freedom & Sig. \\
\hline AFR & 4.00 & 2 & 0.035 \\
Traverse speed & 3.37 & 2 & 0.055 \\
Nozzle Size & 6.61 & 2 & 0.006 \\
\hline
\end{tabular}

Increased AFR resulted in decreased delamination (Fig. 4.11) as observed in Refs. [166] and [188]. The increase in mass flow rate per unit area increased the cutting action of the jet, resulting in reduced side-wall loading via decreased cutting front curvature and kerf taper [45].

Although traverse speed fell slightly outside of the 5\% significance level, its close relation to AFR on cutting front geometry $[182,183,186]$ and trends in previous literature $[83$, 191] suggest that traverse speed is indeed a significant parameter. The results show that increased traverse speed resulted in increased delamination. This trend is also supported by Ramulu and Arola [166], Groppetti and Cattaneo [188], Wong et al. [83], and Dhanawade and Kumar [191]. Increasing traverse speed resulted in a decreased dose and thus reduced jet erosion.

Fig. 4.11 shows that the largest mixing tube produced much greater delamination, consistent with Ref. [42, 101]. This has been attributed to the increase in the power of the jet [3], which resulted in increased volumetric flow rate and increased loading on the cutting front, thereby producing greater crack propagation. The small difference between the 254 and $381 \mu \mathrm{m}$ nozzles under these conditions may be attributed to relatively small crack sizes which could not be distinguished using the moisture uptake method.

The effect of pressure, or equivalently, the jet velocity, on the extent of damage was negligible (Fig. 4.11). This observation should be not be confused with trends observed for piercing operations where increased pressure resulted in increased delamination due to hydrodynamic shock caused by the initial waterjet impact [101] and large blind-hole stagnation pressures [44, 192]. However, Wong et al. [83] and Dhanawade and Kumar [191] have shown that delamination during cutting decreases with increasing pressure, and attributed this to increased particle kinetic energy resulting in reduced cutting front and kerf taper (for a constant traverse speed and AFR), and thus smaller bending of the plies. 


\subsection{FEA Results and Discussion}

\subsubsection{Delamination Onset when AWJ Cutting}

To examine the stresses leading to the onset of delamination, the cohesive zone elements were removed to prevent debonding, while preserving the contact boundary conditions along the ply interfaces in order to resolve the interlaminar tractions. Interlaminar delamination is a result of the out-of-plane interlaminar stresses $\sigma_{z}, \tau_{x z}$, and $\tau_{y z}$ relative to the coordinate system in Fig. 4.5 and Fig. 4.12 [128]. Fig. 4.12 also shows that the maximum values of $\sigma_{z}, \tau_{x z}$, and $\tau_{y z}$ occurred at the interfaces between plies 4-5, 2-3, and 1-2, respectively. These are predicted to be the locations of greatest susceptibility to delamination when AWJ cutting. The interlaminar stresses are highly sensitive to the stacking sequence [193-195] and are largest near free edges $[196,197]$ and decrease rapidly with distance from free boundaries [128]. This is consistent with the numerical results of Fig. 4.12 where the maximum interlaminar stresses (denoted by the flags) prior to debonding occurred at the ply interfaces along the cutting front and the side-wall surfaces. 


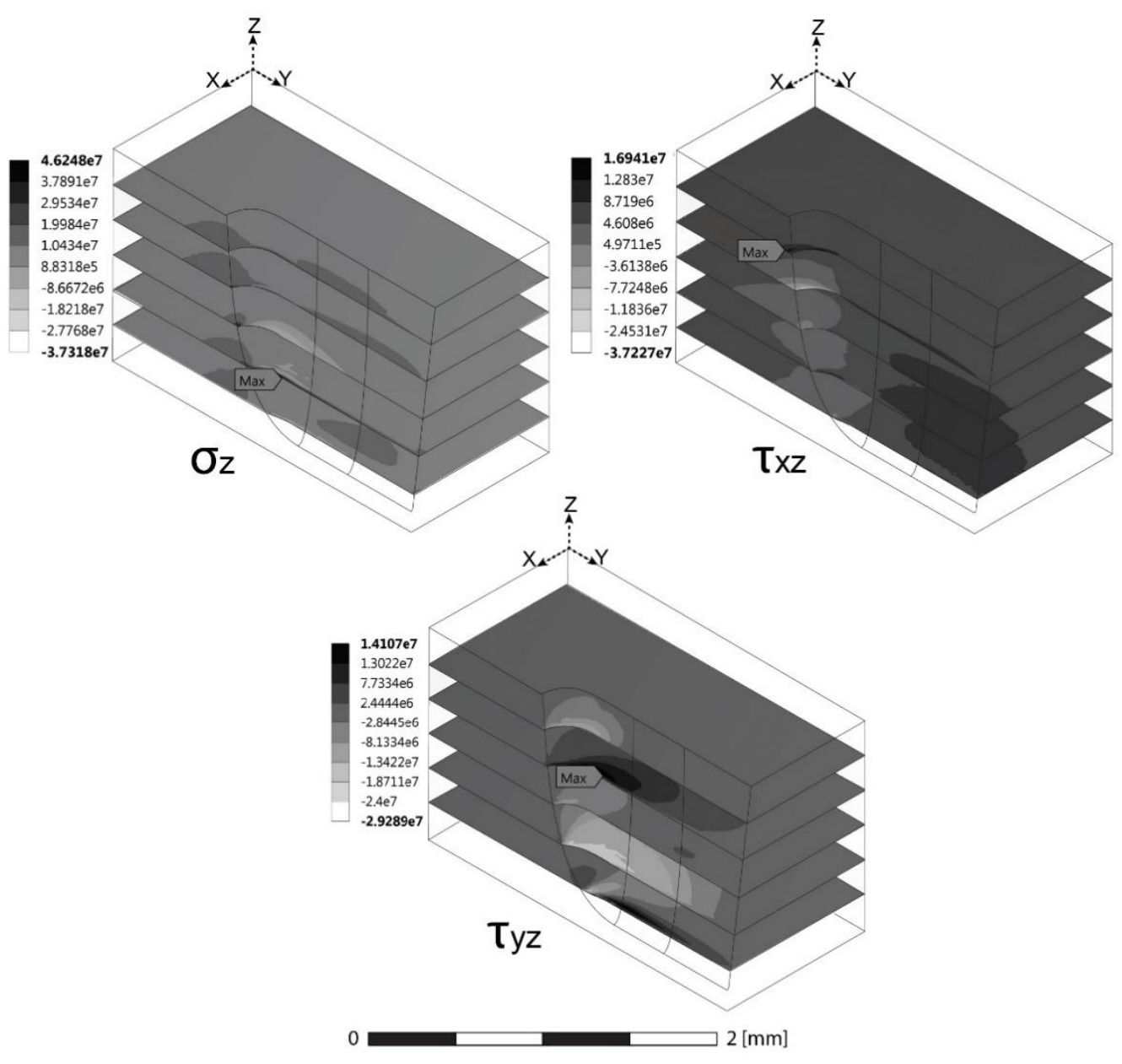

Fig. 4.12 Interlaminar stresses for the fast traverse and smallest nozzle. Flags represent the location of maximum stresses. Fibers have the same orientation as in Fig. 4.1.

The right side of Fig. 4.13 shows the damaged elements (debonding) as predicted by the CZM for the fastest traverse speed with the smallest nozzle. As the normal and tangential tractions grow due to the loading applied by the AWJ, they induce critical displacements where the debonding occurs (Section 4.3.3). Although all three interlaminar stresses contributed to the debonding predicted by the mixed mode CZM, the predicted pattern of debonded elements closely match that of the $\sigma_{z}$ stress field (Fig. 4.13), and in agreement with the observations of Whitney and Browning [198]. The out-of-plane tensile stress $\left(\sigma_{z}\right)$ is associated with a mode I fracture between the plies which occurred more readily than mixed-mode delamination, as reflected by the lower value of $G_{I c}$, compared to $G_{I I c}$, (Table 4.1). This phenomenon is 
represented in the model by a decreased critical normal separation distance $\left(\delta_{c,(n, t)}\right)$ and critical normal traction $\left(\sigma_{c,(n, t)}\right)$ along the CZM elements.

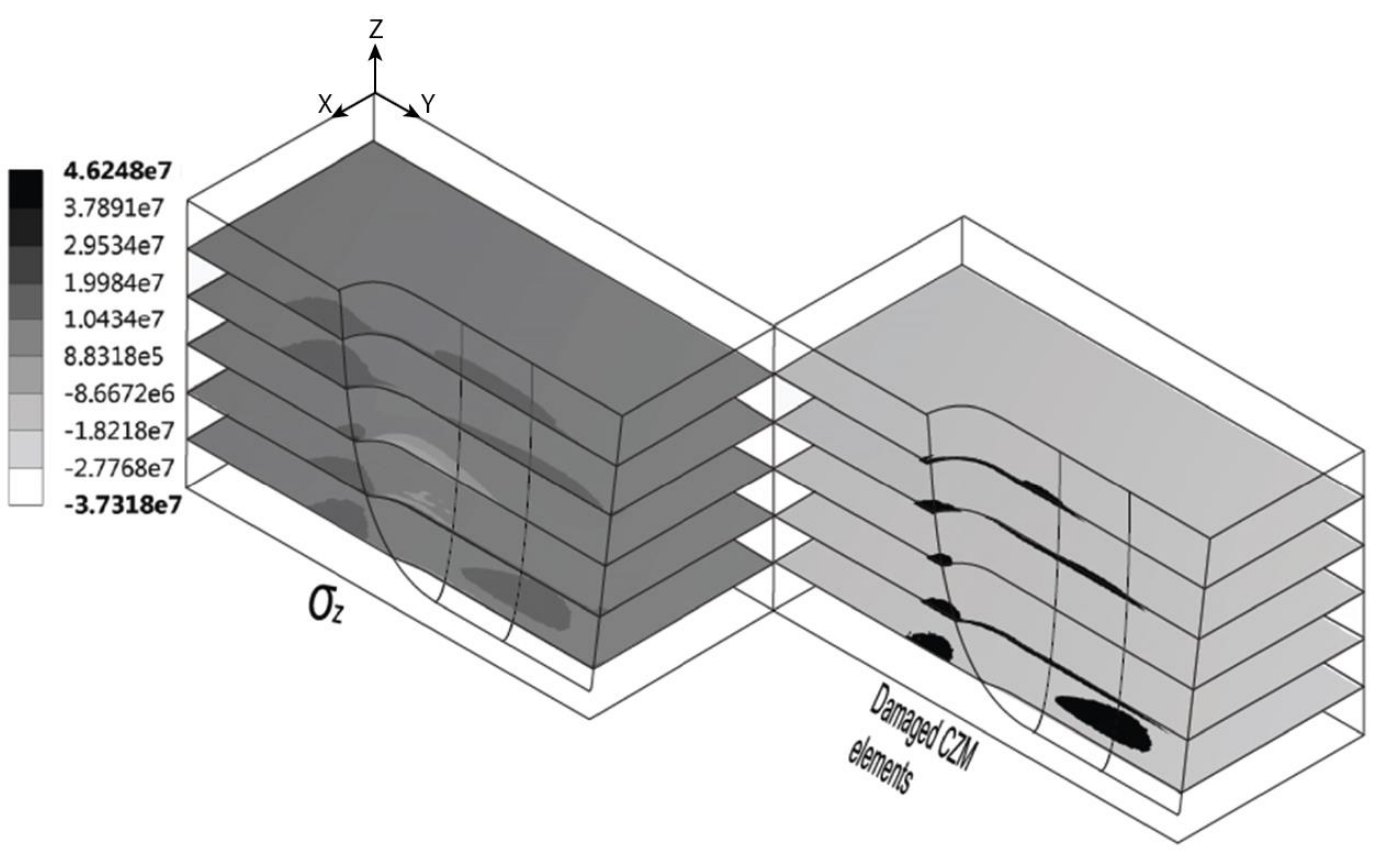

Fig. 4.13 Comparison of predicted $\sigma_{\mathrm{z}}$ interface stresses $(\mathrm{Pa})$ with damaged CZM elements for the fast traverse speed case with the $254 \mu \mathrm{m}$ nozzle.

Fig. 4.13 shows that the numerical results predicted that the largest debonding zone occurred ahead of the crack front along the interface with the bottom-most ply, with comparatively minor delamination along the kerf sidewall. This observation corresponded well with the micro-CT scans of Fig. 4.9 and is consistent with the distribution of the tensile stress in the $z$-direction shown in the left half of the figure. The larger damage ahead of the cutting front, compared to the side wall can be attributed to the larger waterjet loads on the cutting front, as shown in Section 4.4.1. The large oval disbond on the bottom ply beside the cut was also due to the elevated $\sigma_{z}$ in this location as a result of the waterjet loading and the anisotropic behavior of the composite. The FEM predicted smaller amounts of debonding along ply interfaces 1-2 (top ply), 2-3, 4-3 and 4-5, but these were not evident in the micro-CT scans. Likewise, Fig. 4.9 shows delamination encompassing the entire cutting front along the bottom ply, unlike the more 
localized debonding pattern predicted by the FE model in Fig. 4.13. These discrepancies were due of the limitations of the model, which did not consider hydrodynamic pressurization of microcracks by the waterjet, nor the non-homogenous structure of the plies, defects in the layup (e.g., micro-voids, variable ply thicknesses, resin rich areas), and the possibility of intralaminar fracture. The resolution of the CT-scans also limited the number of observable defects to those that were relatively large, particularly in the $x y$-plane.

Finally, Fig. 4.13 shows that minimal damage was predicted at the interface between the central plies 3 and 4 , where the stacking sequence joined two plies with the same fiber orientation, which eliminated the shearing effects caused by different Poisson's ratios and elastic moduli. Thus, these two plies effectively behaved as a single ply, and therefore the $\sigma_{z}$ compression zone along the center ply interface in the left half of Fig. 4.13 can be attributed to the downward loading of the jet, which produced a uniform compression of the interface between plies 3 and 4.. This result was also observed in the micro-CT scans where no delamination was present along the center interface.

The results of the numerical model showed that the variable geometry of the AWJ loading and kerf, and the anisotropic behavior of the laminate generated complex stress fields as shown in Fig. 4.12 and Fig. 4.13. Delamination of these cross-ply laminates during AWJ was found to depend largely on the magnitude and location of the maximum $\sigma_{z}$, rather than $\tau_{x z}$ and $\tau_{y z}$ (Fig. 4.13).

\subsubsection{Comparison of FEA and Moisture Uptake Results}

\subsubsection{Influence of Mixing Tube Diameter}

Although the moisture uptake data was based only a single measurement at a given condition, Fig. 4.14 shows that it broadly followed the same trend with nozzle size as the predicted cumulative area of damaged nodes from the FEM. Based on the model results and coordinate system defined in Fig. 4.5, the increase in delamination is attributed to the increased loading of the jet with increased mixing-tube size. This was examined by calculating the resultant forces acting on the CFD domain shown in Fig. 4.5. For the 762, 381 and $254 \mu \mathrm{m}$ cases, 
these were, respectively: $\left(F_{x}, F_{y}, F_{z}\right)=(-16.8,-21.1,-5.9) \mathrm{N},(-2.3,-3.2,-0.6) \mathrm{N}$, and $(-0.7,-1.0,-$ $0.2)$ N. $F_{y}$ was the largest component of loading in all cases, resulting in ply bending/push out as discussed by Ho-Cheng [44] and Shanmugam et al. [45]. Comparatively, the $762 \mu \mathrm{m}$ nozzle had a significantly larger resultant loading compared to the smaller nozzles, suggesting that smaller nozzles produced less delamination due to reduced reactionary forces, which is consistent with the work of Hashish [98].

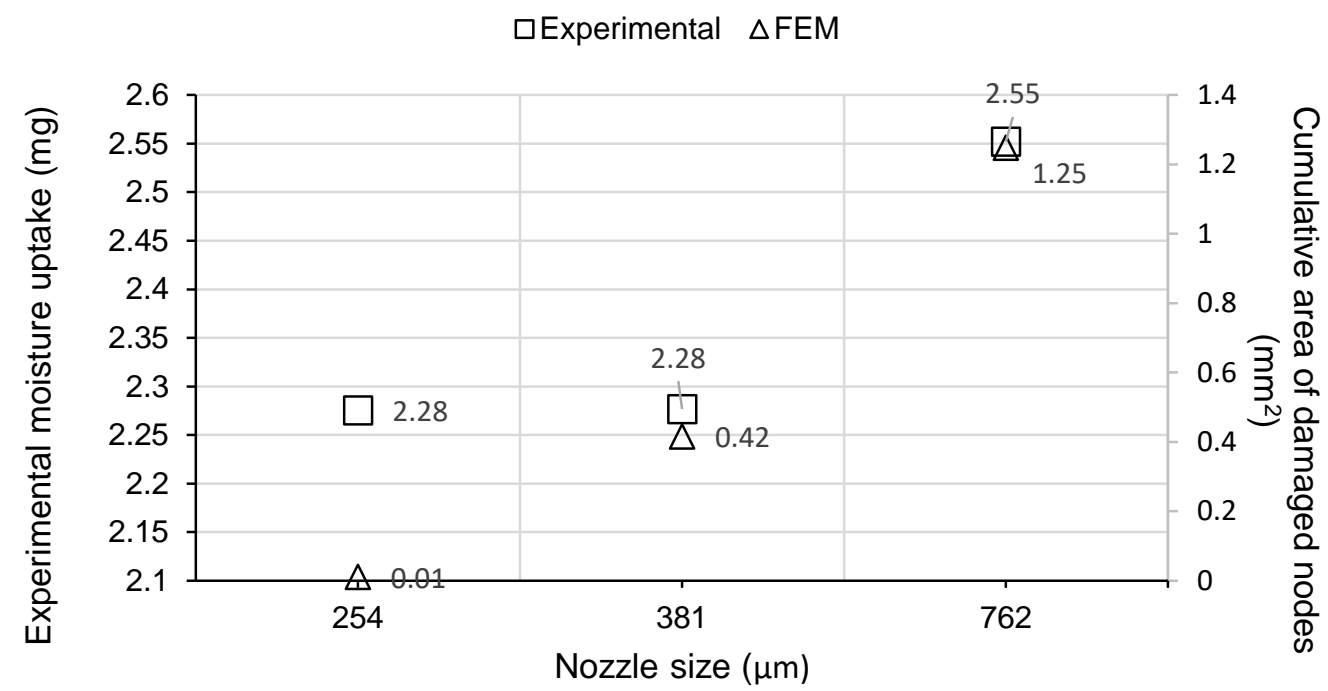

Fig. 4.14 Comparison of experimental damage assessment using moisture uptake and the delamination predicted by the FEA/CZM for three nozzle sizes.

Fig. 4.15 compares the $\sigma_{z}$ stress field and delaminated CZM elements for the 254, 381, and $762 \mu \mathrm{m}$ cases, respectively. The disbonded areas in Fig. 4.15 corresponded to the 'cumulative area of damaged nodes' values shown in Fig. 4.14. Similar to the discussion in Section 4.5.1, the damaged nodes corresponded well to the regions having high $\sigma_{z}$ in all cases. 


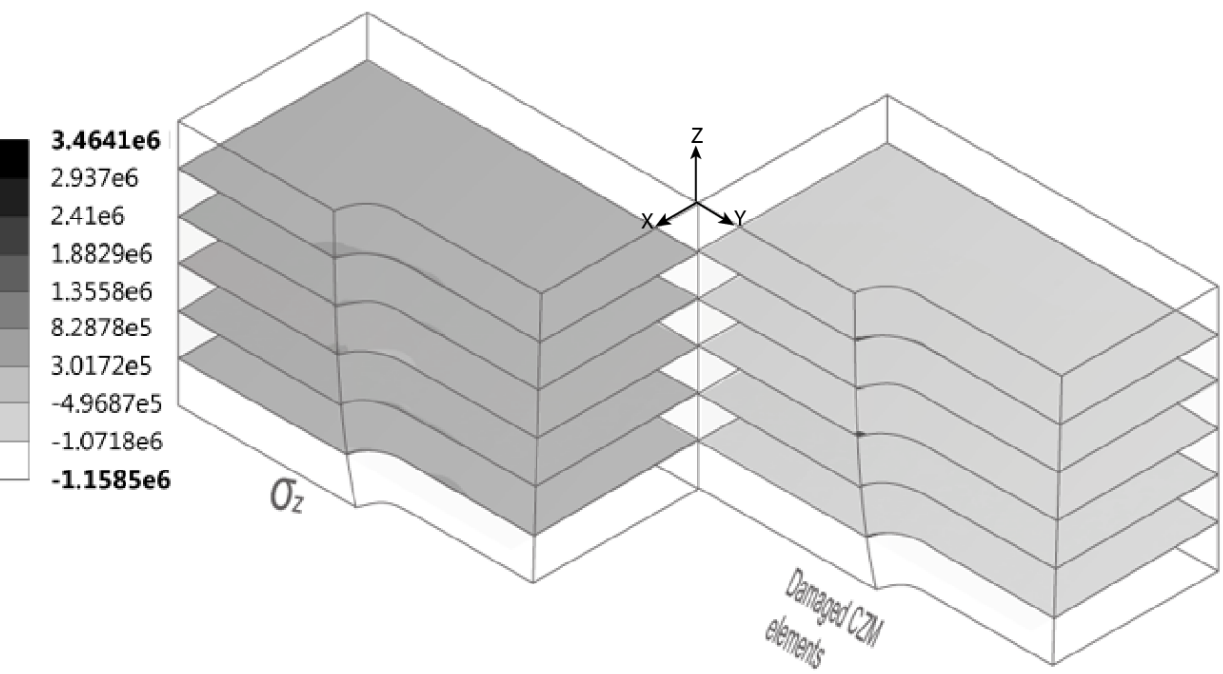

a.

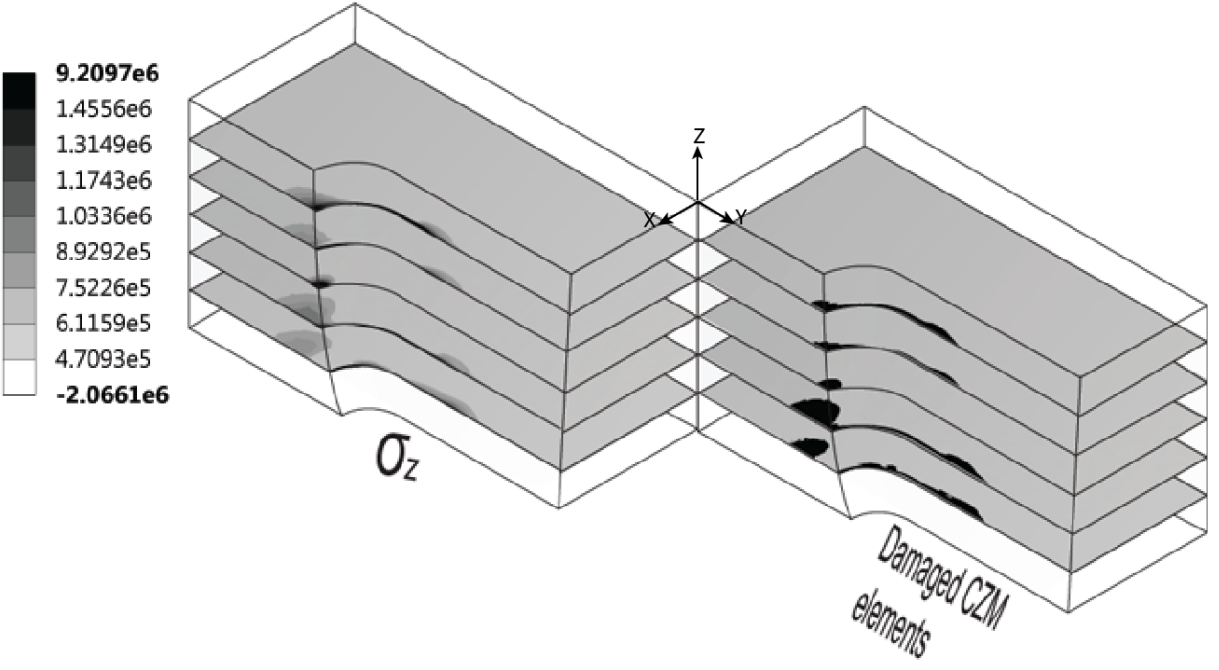

b.

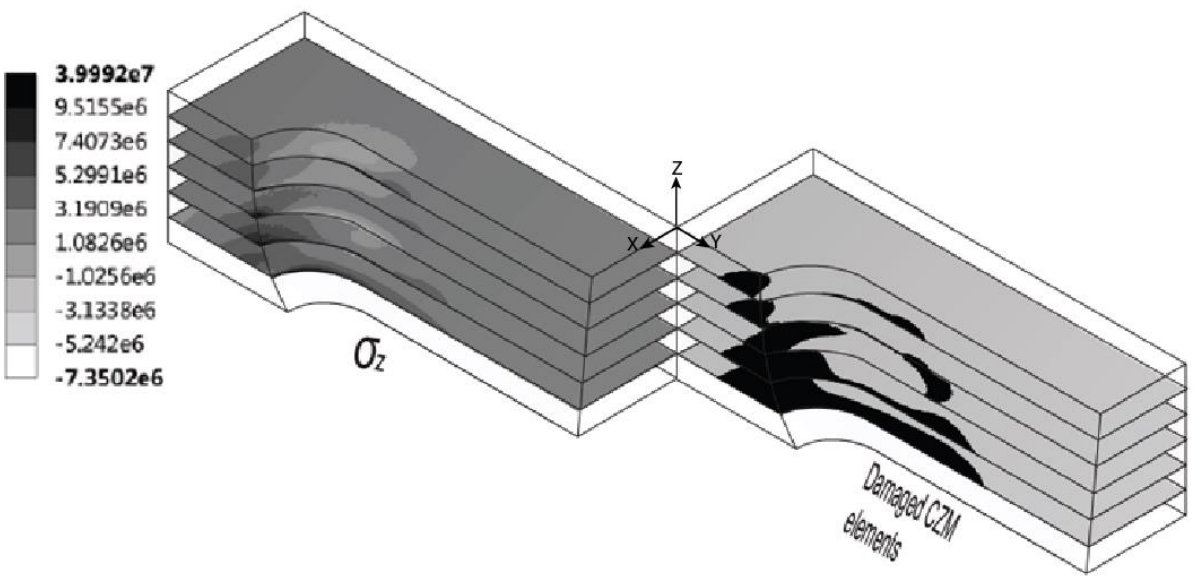

Fig. 4.15 Comparison of predicted $\sigma_{\mathrm{z}}$ stress fields $(\mathrm{Pa})$ at the ply interfaces with delaminated CZM elements for nozzle diameters: a. $254 \mu \mathrm{m}$, b. $381 \mu \mathrm{m}$, and c. $762 \mu \mathrm{m}$, at the fastest traverse speed. 


\subsubsection{Influence of Traverse Speed on Delamination}

Fig. 4.16 compares the moisture uptake results with the total area of debonded CZM nodes for the three traverse speeds, using the smallest nozzle, producing the kerf geometries of Fig. 4.5. The trend of increased delamination with increased traversed speed is supported by the work of Ramulu and Arola [166], Wong et al. [83], Dhanawade and Kumar [191], and Groppetti and Cattaneo [188].

The interlaminar stresses were due to the magnitude and direction of the waterjet loading, which can be quantified by the CFD force vectors. The forces vectors $\left(F_{x}, F_{y}, F_{z}\right)-$ and their resultants $(R)$ - acting on half the cut were: $(-4.3,-23.6,-4.6)(R=24.4) \mathrm{N},(-0.7,-1.0,-0.2)(R=$ 1.2) $\mathrm{N}$, and $(-0.02,1.0,-0.2)(R=1.02) \mathrm{N}$, for the fast, medium and slow cases, respectively. The resultant loading decreased by 20 times from channel milling (i.e., fast case) to through cutting (i.e., medium case). This can be attributed to a majority of the jet volume permeating the exit of the kerf during through cutting, rather than bearing the full load of the jet during milling. Compounding the increased loading with a lengthened moment arm as a result of increased jet lag, resulted in increased bending moment stresses and thus increased damage.

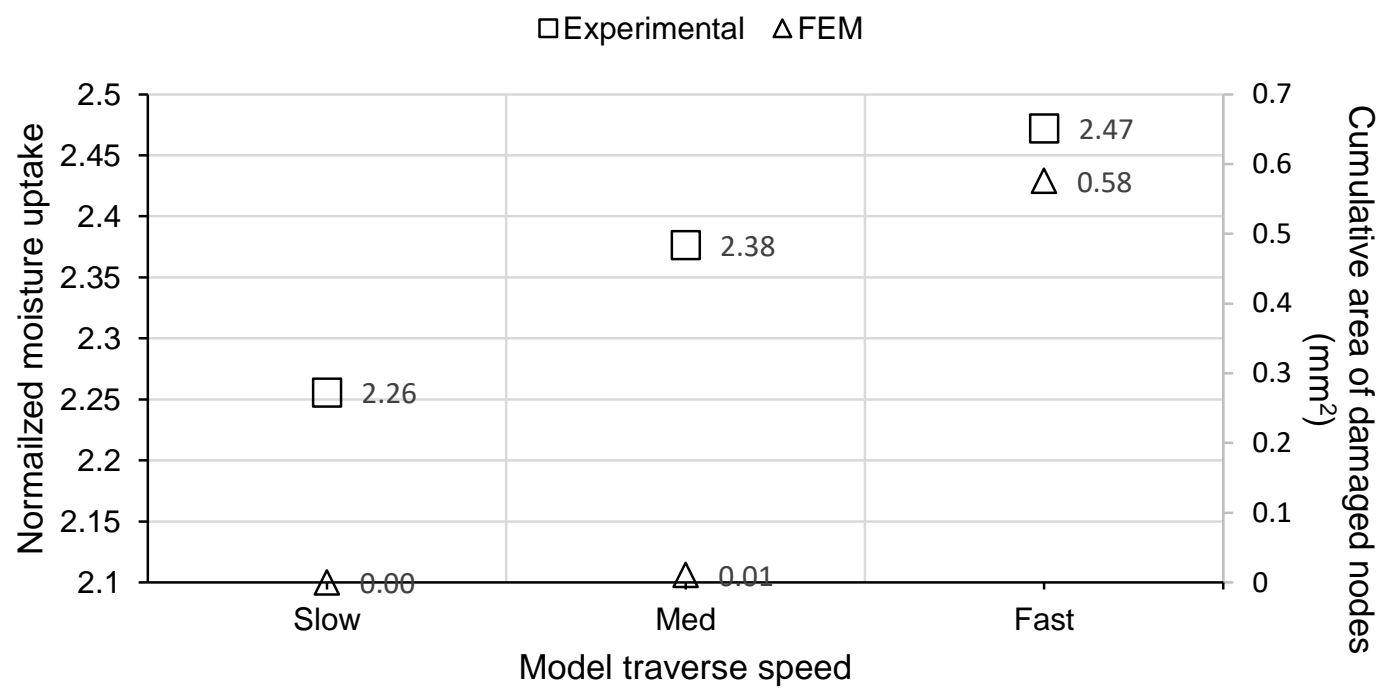

Fig. 4.16 Comparison of experimental damage assessment using moisture uptake and the delamination predicted by the FEA/CZM for three traverse speeds for the 254 nozzle size.

Fig. 4.17 compares the $\sigma_{z}$ stress field and disbonded CZM elements for the slow, medium and fast cases, respectively. The disbonded areas in Fig. 4.17 corresponded to the 'cumulative 
area of damaged nodes' values shown in Fig. 4.16. Similar to the discussion in Section 4.5.1. and 5.2.1, the disbonded nodes corresponded well to the regions of high $\sigma_{z}$ in all cases. Based on the numerical results, the effect of traverse speed on delamination was much less than that of nozzle size.

a.

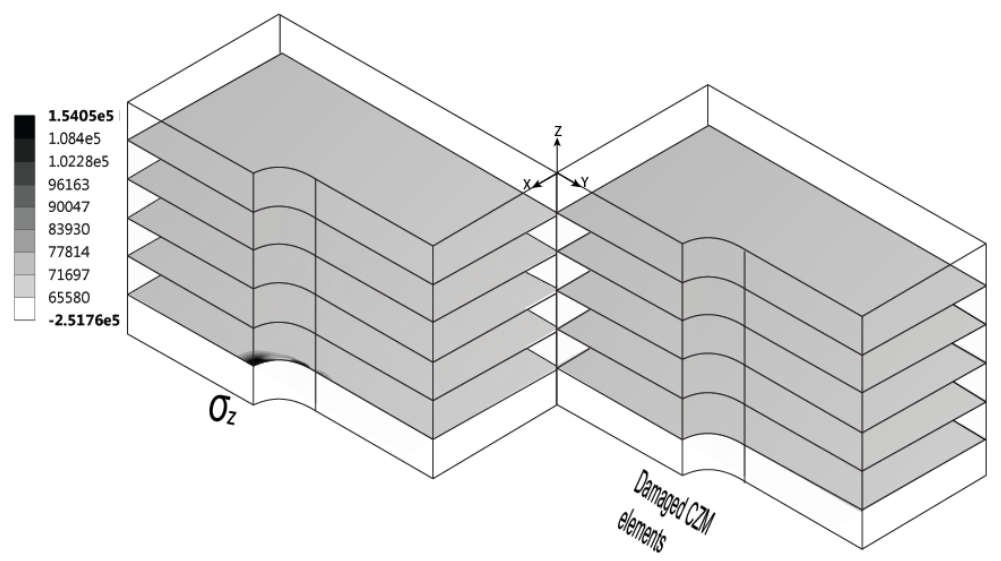

b.
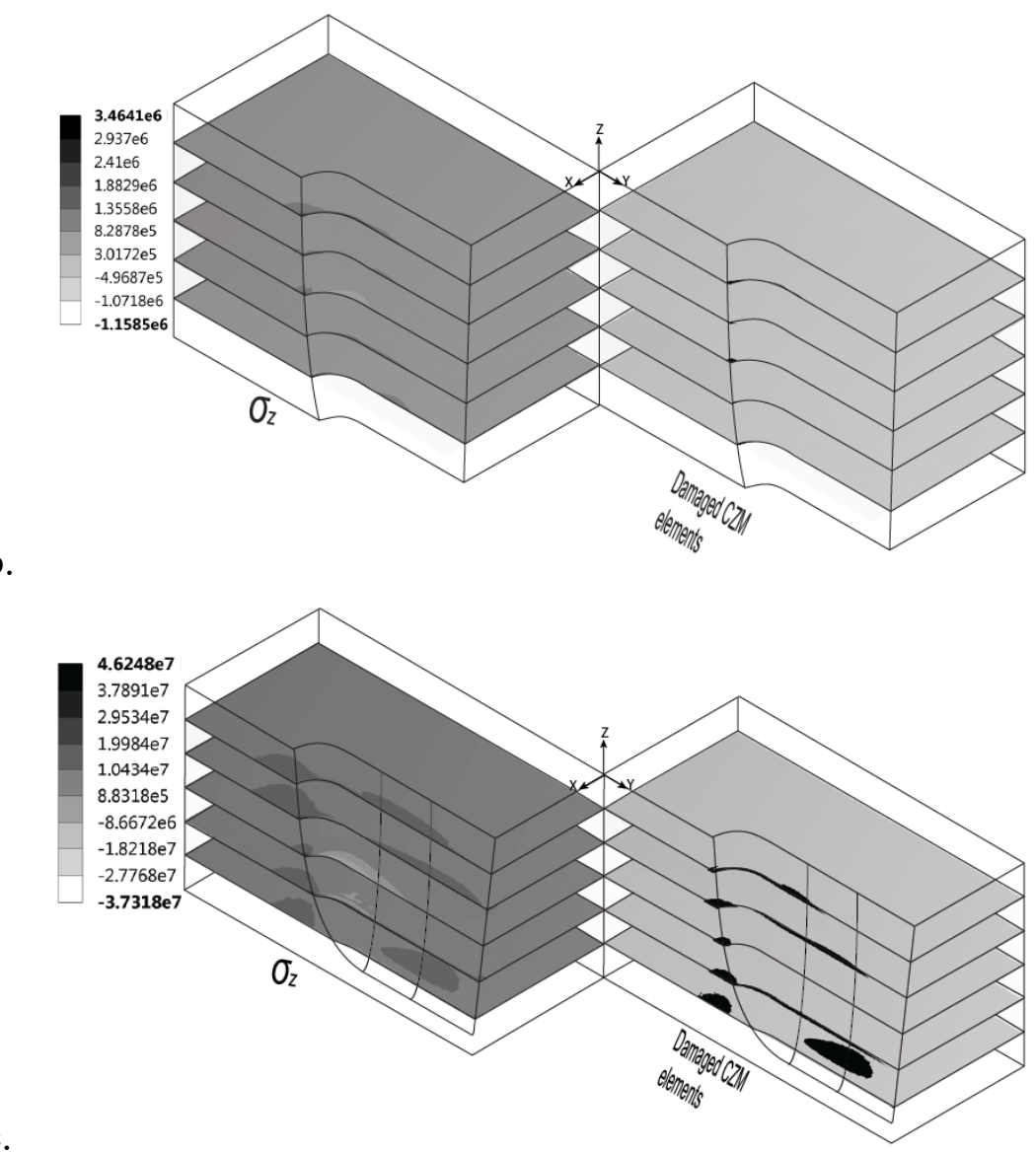

Fig. 4.17 Comparison of predicted $\sigma_{z}$ stress field $(\mathrm{Pa})$ at the ply interfaces with disbonded CZM elements for three traverse speeds: a. slow, b. medium, and c. fast, with the $254 \mu \mathrm{m}$ nozzle. 
A limitation of the CFD/FEM model is its inability to model the effect of AFR. The model did not include discrete particles (i.e. abrasive grains), nor could it model the single particle impact erosion process. However, the work of Wang [199] with alumina ceramics, Kovacevic [200] with AISI 1020 steel, Babu and Chetty [201] with aluminum and Karakurt et al. [202] with granite have shown that traverse speed and AFR share an inverse relationship on the erosive potential of the jet. Thus, it can be inferred that the slow, medium and fast traverse speed model geometries can represent high, medium, and low AFR's, respectively, with a similar but inverse trend to Fig. 4.16 (i.e. increased delamination with decreased AFR).

\subsubsection{Limitations}

The present interpretation of the moisture uptake data assumed that delamination was the only mechanism of damage that could lead to water absorption. However, micro-voids, fiber pullout, and fiber rollout could create features that also absorb water. Therefore, the present inference of the extent of delamination from the moisture uptake results may be an overestimate.

The FEM model focused on crack initiation loading and did not account for the hydrodynamic dynamic pressurization associated with delamination when AWJM [45], and thus it was unable to predict the full extent of the debonding area. Section 4.4.3 to 4.4.5 compare the $\mathrm{CFD} / \mathrm{FEM}$ models to the experimental results of nozzle size, traverse speed, and fiber orientations, respectively. Due to limitations on the control volume used in the model, the effect of SOD, and its effect on jet spreading and deceleration could not be modelled. The effect of AFR (discrete particles) was not incorporated in the FEM model. AFR affects the erosion efficacy of the jet, and thus the cut surface morphology. However, the effect of AFR can be inferred based on the traverse speed results, where both have similar responses on the cutting front and kerf (i.e., as AFR decreases, taper and cutting front curvature increases [37, 180, 181]).

Although the literature and experiments show that interlaminar delamination commonly occurred along the resin-rich interface layer of adjacent plies, the 3D micro-tomography results show that intra-laminar delamination also occurred. The possibility of intralaminar delamination 
was not included in the current model as the CZM elements were restricted to the interfaces between the plies.

\subsection{Summary}

Abrasive water-jet machining of a CF-epoxy laminate is complex due to the anisotropic nature of the material, and the variation of the jet cutting forces, cutting front curvature and kerf taper change with traverse speed and nozzle diameter. Several finite element models were used to model measured AWJ kerf geometries over a range operating conditions (i.e., varying traverse speed and nozzle size). A one-way fluid-structure interaction model was used to simulate the AWJ cutting action, with cohesive zone elements in the structural domain to model crack propagation along the lamina interfaces. The numerical results showed that cutting delamination of the CF-epoxy was primarily dependent on the normal interlaminar stress $\left(\sigma_{z}\right)$, and that a relatively large damage area was observed ahead of the cutting front with less delamination predicted on the side-walls. These results were confirmed by 3D x-ray micro-tomographs of an AWJ cut, which showed larger crack propagation ahead of the cutting front compared to the side wall. The results showed that selecting operating parameters that reduce cutting front curvature (i.e. high pressure, high AFR, small nozzle size and low cutting speed) resulted in decreased delamination.

A jet loading experiment was conducted to compare the difference between through-cutting and trimming as a method to distinguish between side-wall and cutting-front loading by the jet. The results of the loading experiment showed that the cutting front generated larger loadings, and thus increased the tendency for delamination compared to the side wall.

The delamination trends of the numerical model were validated experimentally using a novel moisture uptake testing methodology. The experimental design used a six-factor (pressure, SOD, AFR, traverse speed, mixing-tube size, and fiber orientation), 3-level, Taguchi design of experiments. The results of the experiment and numerical models revealed that increased traverse speed and AFR resulted in decreased moisture uptake (i.e., damage), while increased mixing-tube diameter increased the delamination damage. 


\section{Chapter 5 Summary and Conclusions}

\subsection{Conclusions}

The main conclusions of this dissertation are:

1. $2 \mathrm{D}$ and $3 \mathrm{D}$ surface roughness models were generated to predict surface roughness when AWJ trimming composite substrates. The 2D and 3D surface roughness models had an average error of $10 \%$ and $16 \%$, respectively, over a range of operating conditions. A sensitivity analysis was conducted on the models and showed that particle kinetic properties (i.e. particle velocity and mass) have a more significant effect on surface roughness compared to particle angularity, surface roughness and material dynamic hardness.

2. Delamination as a result of hydraulic shock on anisotropic, carbon-fiber/epoxy laminate was modelled using a two-way FSI model. The experimental delamination trends in the CF-epoxy were analyzed using x-ray micro tomography, and agreed well with the numerical results. The model and experimental results showed that increased jet velocity and increased jet diameter resulted in increased delamination damage. Due to the interdependent relationship between substrate deformation and the critical contact angle of the shock front during impact, the two-way FSI model was able to capture the asymmetric pressure generation, unlike that of isotropic substrates.

3. A one-way FSI model was created to predict delamination when AWJ cutting. The model showed that normal interlaminar stress was predominantly responsible for delamination. The model showed that increased traverse speed (i.e. cutting front curvature) and increased nozzle size, generated higher reactionary forces and thus increased propensity for delamination. The results showed that a majority of the jet loading acted along the cutting front, thus increasing its propensity for delamination. These results were confirmed by x-ray micrographs of AWJ cuts, which showed crack propagation ahead of the cutting front. The effect of AWJ operating parameters on delamination was assessed using a novel moisture uptake methodology and x-ray microtomography, which showed that increased traverse speed and nozzle size had a significant effect on delamination. 


\subsection{Contributions}

The novel contributions of this dissertation can be summarized as:

1. For the first time, the effect of AWJ trimming width on surface roughness was examined. The results showed that roughness vs. trim width followed a periodic relationship, with a minimum surface roughness occurring at approximately half the jet diameter.

2. A novel 2D surface roughness model was generated to predicted kerf surface roughness when AWJ trimming composites. The 2D model used a rigid-plastic particle impact model for predicting crater sizes. These craters were then superimposed to generate a steady-state surface roughness profile that could predict surface roughness within $10 \%$.

3. A novel 3D surface roughness model was generated to predict kerf surface roughness when AWJ trimming composites. 3D conical craters were generated based on the $2 \mathrm{D}$ craters generated by a rigid-plastic particle impact model. The conical craters were then superimposed upon a plane to create a surface capable of predicting surface roughness within $16 \%$.

4. Delamination damage as a result of hydraulic shock during AWJ piercing was visualized and examined using x-ray microtomography, across a range of pressure and nozzle sizes. The results show that the delaminated area was best represented by an ellipse, with greater crack propagation in the longitudinal (fiber) direction, compared to the traverse. It was also shown that increased pressure and nozzle size resulted in increased damage.

5. For the first time, a fluid structure interaction model was developed to simulate the hydraulic shock action of the liquid jet impact on an anisotropic surface, and its subsequent damage generation. The results correlate well with the experimental evidence, with increased pressures and nozzle sizes generating larger hydraulic shocks, and thus increased damage generation.

6. For the first time, a fluid structure interaction model was capable of capturing the interdependent relationship between material deformation and the critical contact angle of the liquid jet, and the subsequent asymmetric pressure distribution generated during the liquid jet impact on anisotropic targets.

7. A novel pressure transducer was developed to capture the difference between the side wall and cutting front loading during AWJ trimming operations. The results showed that 
a majority of the jet loading during cutting was acting along the cutting front, and thus has increased the propensity for delamination when AWJ cutting.

8. Crack propagation ahead of the cutting front was observed via x-ray micrographs of an AWJ cutting front. The results confirm that delamination during AWJ cutting initiates ahead of the cutting front and subsequently envelops it. The delamination ahead of the cutting front is then eroded away as cutting continues, leaving the side kerf delamination remaining. A novel fluid structure interaction model was developed to simulate AWJ cutting of a composite laminate substrate. The model correlated well with the experimental evidence and showed that delamination was highly dependent on the normal interlaminar stresses. 


\subsection{Recommendations for Future Work}

The research presented in this dissertation has the potential to be extended in the following aspects:

I. The current surface roughness model is only applicable for trimming operations. The surface roughness model for trimming could be extended into the cutting regime by considering the effect of secondary particle strikes.

II. The current surface roughness model is not capable of resolving the varying surface roughnesses along the depth of an AWJ cut. Extending the surface roughness model to account for the various surface regions along the kerf, i.e. initial damage region, smooth cutting region, and rough cutting region, and their respective surface roughnesses, would be an asset. This could be accomplished by varying the local particle impact angle based on the kerf shape predicted using previous AWJ surface evolution models.

III. The experimental design of surface roughness used the fundamental variables of particle velocity and dose. However, a majority of AWJ research is discussed in terms of operating parameters such as abrasive flow rate, traverse speed, stand-off distance, etc., which becomes experimentally cumbersome when attempting to account for all operating conditions. Extrapolation of these variables from particle velocity and dose using previous analytical and theoretical models for AWJ fluid flow and erosion would be advantageous for correlating machine operating parameters with material responses, and ease future experimental investigations.

IV. The 3D surface roughness model used conical craters to replicate the surface topology. These conical craters could also be used to generate an erosion model for AWJ machining or used to predict kerf shape. This could be accomplished by 
structuring the superposition of the conical craters based on the particle impact trajectories inside an AWJ (i.e. including the effects of particle distribution inside the jet plume, dose, traverse speed, local particle impact angle and subsequent particle rebound), on a virtual 3D bulk material.

V. Although not discussed in the current dissertation, the liquid jet impact simulation was able to capture the high velocity $(\sim 2000 \mathrm{~m} / \mathrm{s})$ lateral jetting action associated with hydraulic shock. It has been shown in previous literature that the large shear forces generated by the jetting action create localized damage. This damage mechanism has not been modelled or quantified in previous liquid jet impact literature.

VI. Thermal effects were not used in the current impact simulations (Chapter 3), however, highly compressed water is known to increase in temperature and cavitate. The investigation of the thermal effects and cavitation on damage generation of anisotropic materials would be advantageous.

VII. The impact simulation of Chapter 3 only considered a single type of anisotropic target. Thus, it would be of great interest to examine the effect of the hydraulic shock under varying anisotropic material properties. Similarly, several analytical solutions have been generated for liquid jet impact on isotropic materials, yet none exist for anisotropic, presenting a novel research opportunity. This could be accomplished by sectioning the anisotropic substrate into multiple planes, at varying angles, relative to its' global principle directions. The elastic moduli of the sectioned planes (based on local principle directions) could be resolved using stress tensor transformations for bulk anisotropic materials, or classical lamination theory for anisotropic laminates. By assuming a quasi-isotropic material (for a specific sectioned plane), previously developed analytical solutions for liquid impact on elastic targets could be used to extract the plane-specific critical contact angle and hydraulic shock pressure. The solved 2D planes could then be reconstructed about the axis-of-rotation to approximate the hydraulic shock loading of the $3 \mathrm{D}$ bulk anisotropic material. 
VIII. Results suggest there is a critical jet power for which impact induced delamination occurs during AWJ piercing. Although it was beyond the scope of this thesis, developing an analytical or semi-empirical relationship between interfacial material properties and critical impact energy would be valuable for manufacturing purposes. This could be done by taking an energy approach, and relating the impact power of the jet to critical fracture energy release rate of the interfacial layer between bonded lamina.

IX. The effect of hydrodynamic pressurization on delamination during AWJM has yet to be examined. This phenomenon could be resolved using a fluid penetration FEM. 


\section{References}

[1] B. A. Mackey, "How to drill precision holes in reinforced plastics in a hurry," Plastics Engineering, vol. 36, pp. 22-24, 1980.

[2] J. Wang, "Abrasive waterjet machining of polymer matrix composites - cutting performance, erosive process and predictive models," International Journal of Advanced Manufacturing Technology, vol. 15, pp. 757-768, 1999.

[3] H. Liu and E. Schubert, "Micro abrasive-waterjet technology," in Micromachining Techniques for Fabrication of Micro and Nano Structures, M. Kahrizi, Ed., ed: InTech, 2012, pp. 205-233.

[4] L. L. Tirrell, "Method and apparatus for sand blasting," ed: Google Patents, 1940.

[5] D. S. Miller, "Micromachining with abrasive waterjets," Journal of Materials Processing Technology, vol. 149, pp. 37-42, 2004.

[6] N. Haghbin, F. Ahmadzadeh, J. K. Spelt, and M. Papini, "High pressure abrasive slurry jet micro-machining using slurry entrainment," The International Journal of Advanced Manufacturing Technology, vol. 84, pp. 1031-1043, 2016.

[7] A. W. Momber and R. Kovacevic, Principles of abrasive water jet machining. London New York: Springer, 1998.

[8] I. Finnie, "Erosion of surfaces by solid particles," Wear, vol. 3, pp. 87-103, 1960.

[9] J. G. A. Bitter, "A study of erosion phenomena part I," Wear, vol. 6, pp. 5-21, 1963.

[10] J. G. A. Bitter, "A study of Erosion phenomena - Part 2," 1963.

[11] G. L. Sheldon and I. Finnie, "The mechanism of material removal in the erosive cutting of brittle materials," Journal of Engineering for Industry, vol. 88, p. 393, 1966.

[12] D. Aquaro and E. Fontani, "Erosion of ductile and brittle materials," Meccanica, vol. 36, pp. 651-661, 2001.

[13] H. Orbanic and M. Junkar, "Simulation of abrasive water jet cutting process: Part 2. Cellular automata approach," Modelling and Simulation in Materials Science and Engineering, vol. 12, pp. 1171-1184, 2004.

[14] M. Palleda, "A study of taper angles and material removal rates of drilled holes in the abrasive water jet machining process," Journal of Materials Processing Technology, vol. 189, pp. 292-295, 2007.

[15] I. Hutchings, "Mechanisms of the erosion of metals by solid particles," in Erosion: prevention and useful applications, ed: ASTM International, 1979.

[16] I. Hutchings, N. Macmillan, and D. Rickerby, "Further studies of the oblique impact of a hard sphere against a ductile solid," International Journal of Mechanical Sciences, vol. 23, pp. 639-646, 1981.

[17] D. Rickerby and N. Macmillan, "On the oblique impact of a rigid sphere against a rigidplastic solid," International Journal of Mechanical Sciences, vol. 22, pp. 491-494, 1980. 
[18] M. Papini and J. K. Spelt, "Impact of rigid angular particles with fully-plastic targets Part I: Analysis," International Journal of Mechanical Sciences, vol. 42, pp. 991-1006, 2000.

[19] M. Papini and J. K. Spelt, "Impact of rigid angular particles with fully-plastic targets Part II: Parametric study of erosion phenomena," International Journal of Mechanical Sciences, vol. 42, pp. 1007-1025, 2000.

[20] S. Dhar, T. Krajac, D. Ciampini, and M. Papini, "Erosion mechanisms due to impact of single angular particles," Wear, vol. 258, pp. 567-579, 2005.

[21] A. Patnaik, A. Satapathy, S. S. Mahapatra, and R. R. Dash, "A modeling approach for prediction of erosion behavior of glass fiber-polyester composites," Journal of Polymer Research, vol. 15, pp. 147-160, 2008.

[22] S. M. Wiederhorn, "Strength degradation of glass impacted with sharp particles: i, annealed surfaces," American Ceramic Society, Journal, vol. 62, pp. 66-70, 1979.

[23] A. Evans, M. Gulden, and M. Rosenblatt, "Impact damage in brittle materials in the elastic-plastic response regime," in Proceedings of the Royal Society of London A: Mathematical, Physical and Engineering Sciences, 1978, pp. 343-365.

[24] D. Marshall, B. Lawn, and A. Evans, "Elastic/plastic indentation damage in ceramics: the lateral crack system," Journal of the American Ceramic Society, vol. 65, pp. 561-566, 1982.

[25] M. Buijs, "Erosion of glass as modeled by indentation theory," J. Am. Ceram. Soc., vol. 77, pp. 1676-1678, 1994.

[26] J. Y. Sheikh-Ahmad, Machining of Polymer Composites. New York, NY: Springer, 2009.

[27] M. Ramulu and D. Arola, "The influence of abrasive waterjet cutting conditions on the surface quality of grpahite/epoxy laminates," International Journal of Machine Tools and Manurfacturing, vol. 34, pp. 295-313, 1994.

[28] D. Arola and M. Ramula, "A study of kerf characteristics in abrasive waterjet machining of graphite/epoxy composite," Journal of Engineering Materials and Technology, vol. 118, pp. 256-265, 1996.

[29] J. Y. Sheikh-Ahmad, Machining of polymer composites, New York: Springer, 2009.

[30] H. T. Liu, "Hole drilling with abrasive fluidjets," International Journal of Advanced Manufacturing Technology, vol. 32, pp. 942-957, (2007).

[31] M. Simpson, "Abrasive particle study in high pressure water jet cutting," International Journal of Water Jet Technology, vol. 1, pp. 17-28, 1990.

[32] U. Himmelreich and W. Riess, "Laser-velocimetry investigations of the flow in abrasive water jets with varying cutting head geometry," in Proceedings of the 6th American Water Jet Conference, Houston, Texas, 1991.

[33] D. Srinivasu and D. Axinte, "An analytical model for top width of jet footprint in abrasive waterjet milling: a case study on $\mathrm{SiC}$ ceramics," Proceedings of the Insitution of Mechanical Engineers, Part B: Journal of Enigneering Manufacture, vol. 225, pp. 319335, 2011. 
[34] H. Liu, J. Wang, N. Kelson, and R. J. Brown, "A study of abrasive waterjet charachertistics by CFD simulation," Jounral of Materials Processing Technology, vol. 153-154, pp. 488-493, 2004.

[35] M. A. Azmir, A. K. Ahsan, and A. Rahmah, "Effect of abrasive water jet machining parameters on aramid fibre reinforced plastics composite," International Journal of Material Forming, vol. 2, pp. 37-44, 2008.

[36] M. A. Azmir and A. K. Ahsan, "Investigation on glass/epoxy composite surfaces machined by abrasive water jet machining," Journal of Materials Processing Technology, vol. 198, pp. 122-128, 2008.

[37] M. A. Azmir and A. K. Ahsan, "A study of abrasive water jet machining process on glass/epoxy composite laminate," Journal of Materials Processing Tech, vol. 209, pp. 6168-6173, 2009.

[38] J. Wang and D. M. Guo, "A predictive depth of penetration model for abrasive waterjet cutting of polymer matrix composites," Journal of Materials Processing Tech, vol. 121, pp. 390-394, 2002.

[39] M. Shukla and P. B. Tambe, "Predictive modelling of surface roughness and kerf widths in abrasive water jet cutting of Kevlar composites using neural network," International Journal of Machining and Machinability of Materials, vol. 8, pp. 226-246, 01/01/ 2010.

[40] K. Colligan, M. Ramulu, and D. Arola, "Investigation of edge quaity on and ply delamination in abrasive waterjet machining of graphite/epoxy " Machingin of Advanced Composites, vol. 66, pp. 167-185, 1993.

[41] W. Koenig, C. Wulf, H. Grasz, and H. Willerscheid, "Machining of fibre reinforced plastics," Manufacturing Technology, vol. 34, pp. 537-548, 1985.

[42] M. Hashish, "Machining of advanced composites with abrasive-waterjets," in ASME Winter Annual Meeting, Chicago, Illinois, 1988.

[43] H. Ho-Cheng, "A failure analysis of water jet drilling in composite laminates," Int. J. Mach. Tools Manufact., vol. 30, pp. 426-429, 1990.

[44] H. Ho-Cheng, "A failure analysis of water jet drilling in composite laminates," International Journal of Machine Tools and Manufacture, vol. 30, pp. 423-429, 1990.

[45] D. K. Shanmugam, T. Nguyen, and J. Wang, "A study of delamination on graphite/epoxy composites in abrasive waterjet machining," Composites Part A, vol. 39, pp. 923-929, 2008.

[46] M. Papini and J. K. Spelt, "Indentation-induced buckling of organic coatings part I: theory and analysis," International Journal of Mechanical Sciences, vol. 40, pp. 10431059, 1998.

[47] J. Kechagias, G. Petropoulos, and N. Vaxevanidis, "Application of Taguchi design for quality characterization of abrasive water jet machining of TRIP sheet steels," The International Journal of Advanced Manufacturing Technology, vol. 62, pp. 635-643, 2012. 
[48] D. A. Axinte, B. Karpuschewski, M. C. Kong, A. T. Beaucamp, S. Anwar, D. Miller, et al., "High Energy Fluid Jet Machining (HEFJet-Mach): From scientific and technological advances to niche industrial applications," CIRP Annals - Manufacturing Technology, vol. 63, pp. 751-771, 2014.

[49] R. Kovacevic, "Surface texture in abrasive waterjet cutting," Journal of Manufacturing systems, vol. 10, pp. 32-40, 1991.

[50] J. Zeng and A. Henning, "Kerf characterization in abrasive waterjet cutting," in Proceedings of the 2009 WJTA American Waterjet Conference, Houston, TX, Paper, 2009.

[51] F. Müller and J. Monaghan, "Non-conventional machining of particle reinforced metal matrix composites," Journal of Materials Processing Tech, vol. 118, pp. 278-285, 2001.

[52] J. J. R. Jegaraj and N. R. Babu, "A strategy for efficient and quality cutting of materials with abrasive waterjets considering the variation in orifice and focusing nozzle diameter," International Journal of Machine Tools and Manufacture, vol. 45, pp. 1443-1450, 2005.

[53] U. Çaydaş and A. Hasçalık, "A study on surface roughness in abrasive waterjet machining process using artificial neural networks and regression analysis method," Journal of Materials Processing Tech, vol. 202, pp. 574-582, 2008.

[54] H. Liu, T. Gnäupel-Herold, Y. Hovanski, and M. Dahl, "Fatigue performance enhancement of AWJ-machined aircraft aluminum with dry-grit blasting," in Proceedings of American WJTA Conference, Aug, 2009, pp. 18-20.

[55] M. Annoni, F. Arleo, A. Trolli, A. Suarez, A. Alberdi, and R. Tecnalia, "Fine abrasive water jet machining of piezoelectric ceramics: cutting parameters optimization," in 21st Int. Conf. Water Jetting, 2012.

[56] R. H. Mohammad Jafar, J. K. Spelt, and M. Papini, "Numerical simulation of surface roughness and erosion rate of abrasive jet micro-machined channels," Wear, vol. 303, pp. 302-312, 2013.

[57] R. Haj Mohammad Jafar, J. K. Spelt, and M. Papini, "Surface roughness and erosion rate of abrasive jet micro-machined channels: Experiments and analytical model," Wear, vol. 303, pp. 138-145, 2013.

[58] G. Vikram and N. Ramesh Babu, "Modelling and analysis of abrasive water jet cut surface topography," International Journal of Machine Tools and Manufacture, vol. 42, pp. 1345-1354, 2002.

[59] J. P, I. T, and N. D, "Process parameters effect on material removal mechanism and cut quality of abrasive water jet machining," Theoretical and Applied Mechanics, vol. 40, pp. 277-291, 2013.

[60] M. Zohoor and S. Nourian, "Development of an algorithm for optimum control process to compensate the nozzle wear effect in cutting the hard and tough material using abrasive water jet cutting process," The International Journal of Advanced Manufacturing Technology, vol. 61, pp. 1019-1028, 2012. 
[61] P. D. Unde, M. D. Gayakwad, N. G. Patil, R. S. Pawade, D. G. Thakur, and P. K. Brahmankar, "Experimental Investigations into Abrasive Waterjet Machining of Carbon Fiber Reinforced Plastic," Journal of Composites, vol. 2015, 2015.

[62] M. Ramulu and D. Arola, "The influence of abrasive waterjet cutting conditions on the surface quality of graphite/epoxy laminates," International Journal of Machine Tools and Manufacture, vol. 34, pp. 295-313, 1994.

[63] M. Kök, E. Kanca, and Ö. Eyercioğlu, "Prediction of surface roughness in abrasive waterjet machining of particle reinforced MMCs using genetic expression programming," The International Journal of Advanced Manufacturing Technology, vol. 55, pp. 955-968, 2011.

[64] Ž. Ćojbašić, D. Petković, S. Shamshirband, C. W. Tong, S. Ch, P. Janković, et al., "Surface roughness prediction by extreme learning machine constructed with abrasive water jet," Precision Engineering, vol. 43, pp. 86-92, 2016.

[65] N. Haghbin, J. K. Spelt, and M. Papini, " Abrasive waterjet micro-machining of channels in metals: Model to predict high aspect-ratio channel profiles for submerged and unsubmerged machining," Journal of Materials Processing Technology, vol. 222, pp. 399-409, 2013.

[66] Barton. (2013, June). Technical Data and Physical Characteristics for Hard Rock Garnet Abrasives. Available: http://www.barton.com/wp-content/uploads/2013/05/Tech-DataHard-Rock-Garnet_BARTON.pdf

[67] J. Zeng, "Determination of machinability and abrasive cutting properties in AWJ cutting," in Proceedings of 2007 American WJTA Conference and Expo, Houston, Texas, 2007, pp. 19-21.

[68] D. Arola and M. Ramulu, "Material removal in abrasive waterjet machining of metals Surface integrity and texture," Wear, vol. 210, pp. 50-58, 1997.

[69] F. L. Chen and E. Siores, "The effect of cutting jet variation on surface striation formation in abrasive water jet cutting," Journal of Materials Processing Tech, vol. 135, pp. 1-5, 2003.

[70] D. H. Ahmed, J. Naser, and R. T. Deam, "Particles impact characteristics on cutting surface during the abrasive water jet machining: numerical study," Journal of Materials Processing Technology, vol. 232, pp. 116-130, 2016.

[71] C. Narayanan, R. Balz, D. A. Weiss, and K. C. Heiniger, "Modelling of abrasive particle energy in water jet machining," Journal of Materials Processing Technology, vol. 213, pp. 2201-2210, 2013.

[72] N. Haghbin, J. K. Spelt, and M. Papini, "Abrasive waterjet micro-machining of channels in metals: Comparison between machining in air and submerged in water," International Journal of Machine Tools and Manufacture, vol. 88, pp. 108-117, 2015.

[73] J. Schwartzentruber and M. Papini, "Abrasive waterjet micro-piercing of borosilicate glass," Journal of Materials Processing Technology, vol. 219, pp. 143-154, 2015. 
[74] N. Haghbin, J. K. Spelt, and M. Papini, "Abrasive waterjet micro-machining of channels in metals: Model to predict high aspect-ratio channel profiles for submerged and unsubmerged machining," Journal of Materials Processing Technology, vol. 222, pp. 399-409, 2015.

[75] I. M. Hutchings, "A model for the erosion of metals by spherical particles at normal incidence," Wear, vol. 70, pp. 269-281, 1981.

[76] Y. Tirupataiah and G. Sundararajan, "A dynamic indentation technique for the characterization of the high strain rate plastic flow behaviour of ductile metals and alloys," Journal of the Mechanics and Physics of Solids, vol. 39, pp. 243-271, 1991.

[77] H. Getu, J. K. Spelt, and M. Papini, "Conditions leading to the embedding of angular and spherical particles during the solid particle erosion of polymers," Wear, vol. 292-293, pp. 159-168, 2012.

[78] M. Papini, "Organic coating removal by single particle impact," Dissertation/Thesis, ProQuest, UMI Dissertations Publishing, 1999.

[79] H. T. Liu, P. Miles, N. Cooksey, and C. Hibbard, "Measurements of Water-Droplet and Abrasive Speeds in a Ultrahigh-Pressure Abrasive-Waterjets," in 10th American Waterjet Conference, Houston, Texas, 1999.

[80] G. Zhou, M.-C. Leu, E. Geskin, Y. Chung, and J. Chao, "Investigation of topography of waterjet generated surfaces," 1992.

[81] K. Neusen, T. Gores, and R. Amano, "Axial variation of particle and drop velocities downstream from an abrasive water jet mixing tube," in Proceedings of the 12th Conference on Jet Cutting Technology, 1994, pp. 93-103.

[82] R. Balz, R. Mokso, C. Narayanan, D. A. Weiss, and K. C. Heiniger, "Ultra-fast X-ray particle velocimetry measurements within an abrasive water jet," Experiments in Fluids, vol. 54, pp. 1-13, 2013.

[83] I. W. Mm, A. I. Azmi, C. C. Lee, and A. F. Mansor, "Kerf taper and delamination damage minimization of FRP hybrid composites under abrasive water-jet machining," The International Journal of Advanced Manufacturing Technology, vol. 94, pp. 17271744, 2016.

[84] D. K. Shanmugam and S. H. Masood, "An investigation on kerf characteristics in abrasive waterjet cutting of layered composites," Journal of Materials Processing Technology, vol. 209, pp. 3887-3893, 2009.

[85] M. A. Azmir, A. K. Ahsan, and A. Rahmah, "Effect of abrasive water jet machining parameters on aramid fibre reinforced plastics composite," International Journal of Material Forming, vol. 2, pp. 37-44, 2009.

[86] D. K. Shanmugam, J. Wang, and H. Liu, "Minimisation of kerf tapers in abrasive waterjet machining of alumina ceramics using a compensation technique," International Journal of Machine Tools and Manufacture, vol. 48, pp. 1527-1534, 2008.

[87] A. Hascalik, U. Çaydaş, and H. Gürün, "Effect of traverse speed on abrasive waterjet machining of Ti-6Al-4V alloy," Materials and Design, vol. 28, pp. 1953-1957, 2007. 
[88] L. M. Hlaváč, I. M. Hlaváčová, V. Geryk, and Š. Plančár, "Investigation of the taper of kerfs cut in steels by AWJ," The International Journal of Advanced Manufacturing Technology, vol. 77, pp. 1811-1818, 2015.

[89] K. L. Johnson and K. L. Johnson, Contact Mechanics: Cambridge University Press, 1987.

[90] H. Li, "Synthesis, characterization and properties of vinyl ester matrix resins," Dissertation/Thesis, ProQuest Dissertations Publishing, 1998.

[91] J. F. Shackelford, W. Alexander, and CrcnetBase, CRC materials science and engineering handbook vol. 3rd: CRC Press, 2001.

[92] H. Qian, G. Kalinka, K. L. A. Chan, S. G. Kazarian, E. S. Greenhalgh, A. Bismarck, et al., "Mapping local microstructure and mechanical performance around carbon nanotube grafted silica fibres: methodologies for hierarchical composites," Nanoscale - Journal Article, vol. 3, p. 4759, 2011.

[93] S. Hirayama, H. Iwai, and Y. Tanimoto, "Mechanical evaluation of five flowable resin composites by the dynamic micro-indentation method," Journal of dental biomechanics, vol. 5, p. $1758736014533983,2014$.

[94] G. T. Smith, Industrial metrology: surfaces and roundness: Springer, 2002.

[95] R. Kovacevic, R. Mohan, and Y. M. Zhang, "Cutting Force Dynamics as a Tool for Surface Profile Monitoring in AWJ," Journal of Engineering for Industry, vol. 117, p. 340, 1995.

[96] S. Kunaporn, A. Chillman, M. Ramulu, and M. Hashish, "Effect of waterjet formation on surface preparation and profiling of aluminum alloy," Wear, vol. 265, pp. 176-185, 2008.

[97] A. Akkurt, M. K. Kulekci, U. Seker, and F. Ercan, "Effect of feed rate on surface roughness in abrasive waterjet cutting applications," Journal of Materials Processing Tech, vol. 147, pp. 389-396, 2004.

[98] M. Hashish, "Machining of advanced composites with abrasive waterjets," Manufacturing review, vol. 2, pp. 142-150, 1989.

[99] R. Li, H. Ninokata, and M. Mori, "A numerical study of impact force caused by liquid droplet impingement onto a rigid wall," Progress in Nuclear Energy, vol. 53, pp. 881885, 2011.

[100] S. S. Cook, "Erosion by water-hammer," Proceedings of the Royal Society of London. Series A, Containing Papers of a Mathematical and Physical Character, vol. 119, pp. 481-488, 1928.

[101] H. T. Liu and E. Schubert, "Piercing in delicate materials with abrasive-waterjets," The International Journal of Advanced Manufacturing Technology, vol. 42, pp. 263-279, 2008.

[102] L. Huang, J. Folkes, P. Kinnell, and P. H. Shipway, "Mechanisms of damage initiation in a titanium alloy subjected to water droplet impact during ultra-high pressure plain waterjet erosion," Journal of Materials Processing Technology, vol. 212, p. 1906, 2012.

[103] S. Zhang and X. Li, "Theoretical analysis of piercing delicate materials with abrasive water-jet," Journal of the Chinese Institute of Engineers, vol. 33, pp. 1015-1019, 2010. 
[104] A. W. Momber, "Deformation and fracture of rocks due to high-speed liquid impingement," International Journal of Fracture, vol. 130, pp. 683-704, 2004.

[105] P. A. Engel, Impact wear of materials vol. 2: Elsevier, 1978.

[106] J. Field, M. Farhat, P. Kobel, D. Obreschkow, M. Tinguely, A. de Bosset, et al., "Confined shocks inside isolated liquid volumes: A new path of erosion?," Physics of Fluids, vol. 23, pp. 101702-101702-4, 2011.

[107] F. J. Heymann, "High-speed impact between a liquid drop and a solid surface," Journal of Applied Physics, vol. 40, pp. 5113-5122, 1969.

[108] M. B. Lesser, "Analytic solutions of liquid-drop impact problems," Proceedings of the Royal Society of London. Series A, Mathematical and Physical Sciences, vol. 377, pp. 289-308, 1981.

[109] J. E. Field, M. B. Lesser, and J. P. Dear, "Studies of two-dimensional liquid-wedge impact and their relevance to liquid-drop impact problems," Proceedings of the Royal Society of London. Series A, Mathematical and Physical Sciences, vol. 401, pp. 225-249, 1985.

[110] C.-Y. Hsu, C.-C. Liang, T.-L. Teng, and A.-T. Nguyen, "A numerical study on highspeed water jet impact," Ocean Engineering, vol. 72, pp. 98-106, 2013.

[111] T. Obara, N. K. Bourne, and J. E. Field, "Liquid-jet impact on liquid and solid surfaces," Wear, vol. 186-187, pp. 388-394, 1995.

[112] F. P. Bowden and J. H. Brunton, "The deformation of solids by liquid impact at supersonic speeds," Proceedings of the Royal Society of London. Series A, Mathematical and Physical Sciences, vol. 263, pp. 433-450, 1961.

[113] J. P. Dear and J. E. Field, "High-speed photography of surface geometry effects in liquid/solid impact," Journal of Applied Physics, vol. 63, p. 1015, 1988.

[114] N. K. Bourne, "On impacting liquid jets and drops onto polymethylmethacrylate targets," Proceedings of the Royal Society A: Mathematical, Physical and Engineering Science, vol. 461, pp. 1129-1145, 2005.

[115] J. E. Field and M. B. Lesser, "On the Mechanics of High Speed Liquid Jets," Proceedings of the Royal Society of London. Series A, Mathematical and Physical Sciences, vol. 357, pp. 143-162, 1977.

[116] A. A. Aganin and T. S. Guseva, "Numerical simulation of impact of a jet on a wall," Mathematical Models and Computer Simulations, vol. 9, pp. 623-635, 2017.

[117] M. B. Lesser and J. E. Field, "The impact of compressible liquids," Annual Review of Fluid Mechanics, vol. 15, pp. 97-122, 1983.

[118] H. Jeong and Y.-S. Jang, "Wavelet analysis of plate wave propagation in composite laminates," Composite Structures, vol. 49, pp. 443-450, 2000.

[119] D. H. Hurley and J. B. Spicer, "An investigation of the effects of material anisotropy and heterogeneity on pulsed, laser-generated acoustic signals," IEEE Transactions on Ultrasonics, Ferroelectrics and Frequency Control, vol. 46, pp. 1387-1395, 1999.

[120] J. Zukas, Introduction to hydrocodes vol. 49: Elsevier, 2004. 
[121] D. A. Gorham and J. E. Field, "The failure of composite materials under high-velocity liquid impact," Journal of Physics D: Applied Physics, vol. 9, p. 1529, 1976.

[122] C. S. Alexander, C. Key, and S. Schumacher, "Improved understanding of the dynamic response in anisotropic directional composite materials through the combination of experiments and modeling," in Journal of Physics: Conference Series, 2014, p. 112002.

[123] J. H. Brunton, "High speed liquid impact," Philosophical Transactions of the Royal Society of London. Series A, Mathematical and Physical Sciences, vol. 260, pp. 79-85, 1966.

[124] N. K. Bourne, "On stress wave interactions in liquid impact," Wear, vol. 258, pp. 588595, 2005.

[125] R. J. Hand, J. E. Field, and D. Townsend, "The use of liquid jets to simulate angled drop impact," Journal of Applied Physics, vol. 70, pp. 7111-7118, 1991.

[126] J. Wang, "Particle velocity models for ultra-high pressure abrasive waterjets," Journal of Materials Processing Technology, vol. 209, pp. 4573-4577, 2009.

[127] S. Dehkhoda and N. K. Bourne, "Production of a high-velocity water slug using an impacting technique," The Review of scientific instruments, vol. 85, p. 025109, 2014.

[128] B. D. Agarwal, L. J. Broutman, and K. Chandrashekhara, Analysis and Performance of Fiber Composites. Hoboken, New Jersey: Wiley, 2006.

[129] P. H. Geubelle and J. S. Baylor, "Impact-induced delamination of composites: a 2D simulation," Composites Part B, vol. 29, pp. 589-602, 1998.

[130] J. N. Johnson, "Single-Particle Model of a Solid: The Mie-Grüneisen Equation," American Journal of Physics, vol. 36, pp. 917-919, 1968.

[131] S. Wilkinson and W. Reynolds, "The propagation of ultrasonic waves in carbon-fibrereinforced plastics," Journal of Physics D: Applied Physics, vol. 7, p. 50, 1974.

[132] G. Fernlund, M. Papini, D. McCammond, and J. K. Spelt, "Fracture load predictions for adhesive joints," Composites Science and Technology, vol. 51, pp. 587-600, 1994.

[133] S. Azari, M. Eskandarian, M. Papini, J. A. Schroeder, and J. K. Spelt, "Fracture load predictions and measurements for highly toughened epoxy adhesive joints," Engineering Fracture Mechanics, vol. 76, pp. 2039-2055, 2009.

[134] X. X. Xu, A. D. Crocombe, and P. A. Smith, "Mixed-mode fatigue and fracture behaviour of joints bonded with either filled or filled and toughened adhesive," International Journal of Fatigue, vol. 17, pp. 279-286, 1995.

[135] ASTM, "ASTM D792-13, Standard test methods for density and specific gravity (relative density) of plastics by displacement," ed. West Conshohocken, PA: ASTM, 2013.

[136] ASTM, "ASTM D3039 / D3039M-14, Standard test method for tensile properties of polymer matrix composite materials, ," ed. West Conshohocken, PA, 2014.

[137] M. Hashish, "Inside AWJ Nozzles," in 2003 WJTA American Waterjet Conference, Houston, Texas, 2003, pp. 243-260. 
[138] Z. Yong and R. Kovacevic, "Effects of water-mixture film on impact contact in abrasive waterjet machining," International Journal of Mechanical Sciences, vol. 39, pp. 729-739, 1997.

[139] F. Aymerich, F. Dore, and P. Priolo, "Prediction of impact-induced delamination in cross-ply composite laminates using cohesive interface elements," Composites Science and Technology, vol. 68, pp. 2383-2390, 2008.

[140] H. Liu, J. Wang, N. Kelson, and R. J. Brown, "A study of abrasive waterjet characteristics by CFD simulation," Journal of Materials Processing Technology, vol. 153-154, pp. 488-493, 2004.

[141] K. Kowsari, H. Nouraei, B. Samareh, M. Papini, and J. K. Spelt, "CFD-aided prediction of the shape of abrasive slurry jet micro-machined channels in sintered ceramics," Ceramics International, vol. 42, pp. 7030-7042, 2016.

[142] P. Coray, B. Jurisevic, M. Junkar, and K. C. Heiniger, "Measurements on 5: 1 scale abrasive water jet cutting head models," in Proceedings of the 6th International Conference on Management of Innovative Technologies MIT, 2003, pp. 87-102.

[143] O. Ubbink and R. I. Issa, "A method for capturing sharp fluid interfaces on arbitrary meshes," Journal of Computational Physics, vol. 153, pp. 26-50, 1999.

[144] B. E. Launder and D. B. Spalding, Lectures in mathematical models of turbulence: Academic Press, 1972.

[145] N. K. Bourne, T. Obara, and J. E. Field, "High-speed photography and stress gauge studies of jet impact upon surfaces," Philosophical Transactions: Mathematical, Physical and Engineering Sciences, vol. 355, pp. 607-623, 1997.

[146] T. Obara, N. K. Bourne, and Y. Mebar, "The construction and calibration of an inexpensive PVDF stress gauge for fast pressure measurements," Measurement Science and Technology, vol. 6, p. 345, 1995.

[147] P. Kohnke, "Theory reference for the mechanical APDL and mechanical applications," Ansys Inc, release, vol. 12, 2009.

[148] X. Xu, M. R. Wisnom, X. Li, and S. R. Hallett, "A numerical investigation into size effects in centre-notched quasi-isotropic carbon/epoxy laminates," Composites Science and Technology, vol. 111, pp. 32-39, 2015.

[149] A. Atas, G. F. Mohamed, and C. Soutis, "Modelling delamination onset and growth in pin loaded composite laminates," Composites Science and Technology, vol. 72, pp. 10961101, 2012.

[150] L. M. P. Durão, M. F. S. F. de Moura, and A. T. Marques, "Numerical simulation of the drilling process on carbon/epoxy composite laminates," Composites Part A, vol. 37, pp. 1325-1333, 2006.

[151] D. C. Pham, X. S. Sun, V. B. C. Tan, B. Chen, and T. E. Tay, "Progressive failure analysis of scaled double-notched carbon/epoxy composite laminates," International Journal of Damage Mechanics, vol. 21, pp. 1154-1185, 2012. 
[152] A. M. G. Pinto, R. D. S. G. Campilho, M. F. S. F. de Moura, and I. R. Mendes, "Numerical evaluation of three-dimensional scarf repairs in carbon-epoxy structures," International Journal of Adhesion and Adhesives, vol. 30, pp. 329-337, 2010.

[153] N. Hu, Y. Zemba, T. Okabe, C. Yan, H. Fukunaga, and A. M. Elmarakbi, "A new cohesive model for simulating delamination propagation in composite laminates under transverse loads," Mechanics of Materials, vol. 40, pp. 920-935, 2008.

[154] O. Isbilir and E. Ghassemieh, "Three-dimensional numerical modelling of drilling of carbon fiber-reinforced plastic composites," Journal of Composite Materials, vol. 48, pp. 1209-1219, 2014.

[155] L. Lampani, "Finite element analysis of delamination of a composite component with the cohesive zone model technique," Engineering Computations, vol. 28, pp. 30-46, 2011.

[156] Y. Shi, C. Pinna, and C. Soutis, "Interface cohesive elements to model matrix crack evolution in composite laminates," Applied Composite Materials, vol. 21, pp. 57-70, 2014.

[157] S. S. Shams and R. F. El-Hajjar, "Effects of scratch damage on progressive failure of laminated carbon fiber/epoxy composites," International Journal of Mechanical Sciences, vol. 67, pp. 70-77, 2013.

[158] X. Xu, A. Qing, Y. B. Gan, and Y. P. Feng, "Effective properties of fiber composite materials," Journal of Electromagnetic Waves and Applications, vol. 18, pp. 649-649, 2004.

[159] A. Turon, C. G. Dávila, P. P. Camanho, and J. Costa, "An engineering solution for mesh size effects in the simulation of delamination using cohesive zone models," Engineering Fracture Mechanics, vol. 74, pp. 1665-1682, 2007.

[160] S. P. V. Nadimpalli and J. K. Spelt, "Fracture load prediction of lead-free solder joints," Engineering Fracture Mechanics, vol. 77, pp. 3446-3461, 2010.

[161] A. Nourani, S. Akbari, and J. K. Spelt, "Fracture load prediction of BGA solder joints: Cohesive zone modeling and experimental verification," International Journal of Solids and Structures, vol. 90, pp. 30-44, 2016.

[162] M. Lesser and J. Field, "Studies in shock waves, liquid impact, jets and cavitation," in Shock Waves: Proceedings of the 18th International Symposium on Shock Waves, Held at Sendai, Japan 21-26 July 1991, K. Takayama, Ed., ed Berlin, Heidelberg: Springer Berlin Heidelberg, 1992, pp. 61-72.

[163] E. Pessis, R. Campagna, J.-M. Sverzut, F. Bach, M. Rodallec, H. Guerini, et al., "Virtual monochromatic spectral imaging with fast kilovoltage switching: reduction of metal artifacts at CT," Radiographics : a review publication of the Radiological Society of North America, Inc, vol. 33, pp. 573-583, 2013.

[164] P. Deconinck, J. Capelle, V. Bouchart, P. Chevrier, and F. Ravailler, "Delamination propagation analysis in tufted carbon fibre-reinforced plastic composites subjected to high-velocity impact," Journal of Reinforced Plastics and Composites, vol. 33, pp. 1353$1363,2014$. 
[165] D. G. Smith and R. Kinslow, "Pressure due to high-velocity impact of a water jet: The transient pressure distribution across the impact area of a small high-velocity water jet is studied by means of a pressure-transducer technique," Experimental Mechanics, vol. 16, pp. 21-25, 1976.

[166] M. Ramulu and D. Arola, "Water jet and abrasive water jet cutting of unidirectional graphite/epoxy composite," Composites, vol. 24, pp. 299-308, 1993.

[167] M. Saleem, H. Bougherara, L. Toubal, F. Cénac, and R. Zitoune, "Analysis of Stresses in CFRP Composite Plates Drilled with Conventional and Abrasive Water Jet Machining," Materials Science Forum, vol. 763, pp. 127-143, 2013.

[168] A. Dhanawade and S. Kumar, "Experimental study of delamination and kerf geometry of carbon epoxy composite machined by abrasive water jet," Journal of Composite Materials, vol. 51, pp. 3373-3390, 2017.

[169] K. Colligan, M. Ramulu, and D. Arola, "Investigation of edge quality and ply delamination in abrasive waterjet machining of graphite/epoxy," in Proceedings of the 1993 ASME Winter Annual Meeting, 1993.

[170] M. Ramulu, "Machining and surface integrity of fibre-reinforced plastic composites," Sadhana, vol. 22, pp. 449-472, 1997.

[171] J. Zeng, J. Olsen, C. Olsen, and B. Guglielmetti, "Taper-free abrasive waterjet cutting with a tilting head," in Proceedings of the 2005 American Waterjet Conference, 2005, pp. 21-23.

[172] G. Davis, "Evaluating adhesive bonds with carbon-composites using electrochemical impedance spectroscopy," in 224th Electrochemical Society Meeting, San Franscisco, USA, 2013.

[173] G. D. Davis, M. J. Rich, and L. T. Drzal, "Monitoring moisture uptake and delamination in cfrp-reinforced concrete structures with electrochemical impedance sensors," Journal of Nondestructive Evaluation, vol. 23, pp. 1-9, 2004.

[174] R. C. Glass, S. R. Taylor, G. L. Cahen, and G. E. Stoner, "Electrochemical impedance spectroscopy as a method to nondestructively monitor simulated in-service damage in a carbon fiber reinforced plastic," Journal of Nondestructive Evaluation, vol. 6, pp. 181188, 1987.

[175] Y. Shimamura, T. Urabe, A. Todoroki, and H. Kobayashi, "Electrical impedance change method for moisture absorption monitoring of CFRP," Advanced Composite Materials, vol. 13, pp. 297-310, 2004.

[176] D. J. Boll, W. D. Bascom, and B. Motiee, "Moisture absorption by structural epoxymatrix carbon-fiber composites," Composites Science and Technology, vol. 24, pp. 253273, 1985.

[177] C. Soutis and D. Turkmen, "Moisture and temperature effects of the compressive failure of cfrp unidirectional laminates," Journal of Composite Materials, vol. 31, pp. 832-849, 1997. 
[178] J. Zhou and J. P. Lucas, "The effects of a water environment on anomalous absorption behavior in graphite/epoxy composites," Composites Science and Technology, vol. 53, pp. 57-64, 1995.

[179] B. G. Kumar, R. P. Singh, and T. Nakamura, "Degradation of carbon fiber-reinforced epoxy composites by ultraviolet radiation and condensation," Journal of Composite materials, vol. 36, pp. 2713-2733, 2002.

[180] S. Zhang, Y. Wu, and S. Wang, "An exploration of an abrasive water jet cutting front profile," The International Journal of Advanced Manufacturing Technology, vol. 80, pp. 1685-1688, 2015.

[181] L. M. Hlaváć, "Investigation of the abrasive water jet trajectory curvature inside the kerf," Journal of Materials Processing Technology, vol. 209, pp. 4154-4161, 2009.

[182] H. Orbanic and M. Junkar, "Analysis of striation formation mechanism in abrasive water jet cutting," Wear, vol. 265, pp. 821-830, 2008.

[183] R. T. Deam, E. Lemma, and D. H. Ahmed, "Modelling of the abrasive water jet cutting process," Wear, vol. 257, pp. 877-891, 2004.

[184] H. C. Wu and W. C. Yeh, "On the experimental determination of yield surfaces and some results of annealed 304 stainless steel," International Journal of Plasticity, vol. 7, pp. 803-826, 1991.

[185] M. Jerman, J. Valentinčič, A. Lebar, and H. Orbanić, "The study of abrasive water jet cutting front development using a two-dimensional cellular automata model," Strojniški vestnik-Journal of Mechanical Engineering, vol. 61, pp. 292-302, 2015.

[186] M. Hashish, "Visualization of the abrasive-waterjet cutting process," Experimental Mechanics, vol. 28, pp. 159-169, 1988.

[187] J. Zeng, "Mechanisms of brittle material erosion associated with high-pressure abrasive waterjet processing: A modeling and application study," Dissertation/Thesis, University of Rhode Island, 1992.

[188] R. Groppetti and A. Cattaneo, "Model for hydro and hydro-abrasive jet machining of carbon fiber-reinforced plastic composites," NIST Spec. Publ., 847 pp., vol. 397, 1993.

[189] A. Alberdi, T. Artaza, A. Suárez, A. Rivero, and F. Girot, "An experimental study on abrasive waterjet cutting of CFRP/Ti6Al4V stacks for drilling operations," The International Journal of Advanced Manufacturing Technology, vol. 86, pp. 691-704, 2016.

[190] G. Cosansu and C. Cogun, "An investigation on use of colemanite powder as abrasive in abrasive waterjet cutting (AWJC)," Journal of Mechanical Science and Technology, vol. 26, pp. 2371-2380, 2012.

[191] A. Dhanawade and S. Kumar, "Experimental study of delamination and kerf geometry of carbon epoxy composite machined by abrasive water jet," Journal of Composite Materials, p. 2199831668895, 2017. 
[192] K. Phapale, R. Singh, S. Patil, and R. K. P. Singh, "Delamination characterization and comparative assessment of delamination control techniques in abrasive water jet drilling of CFRP," Procedia Manufacturing, vol. 5, pp. 521-535, 2016.

[193] N. J. Pagano and R. B. Pipes, "The Influence of Stacking Sequence on Laminate Strength," Journal of Composite Materials, vol. 5, pp. 50-57, 1971.

[194] J. Whitney, "Free-edge effects in the characterization of composite materials," in Analysis of the Test Methods for High Modulus Fibers and Composites, ed: ASTM International, 1973.

[195] R. B. Pipes, B. Kaminski, and N. Pagano, "Influence of the free edge upon the strength of angle-ply laminates," in Analysis of the Test Methods for High Modulus Fibers and Composites, ed: ASTM International, 1973.

[196] R. B. Pipes and N. J. Pagano, "Interlaminar stresses in composite laminates under uniform axial extension," Journal of Composite Materials, vol. 4, pp. 538-548, 1970.

[197] R. B. Pipes, "Boundary layer effects in composite laminates," Fibre Science and Technology, vol. 13, pp. 49-71, 1980.

[198] J. M. Whitney and C. E. Browning, "Free-edge delamination of tensile coupons," Journal of Composite Materials, vol. 6, pp. 300-303, 1972.

[199] J. Wang, "Predictive depth of jet penetration models for abrasive waterjet cutting of alumina ceramics," International Journal of Mechanical Sciences, vol. 49, pp. 306-316, 2007.

[200] N. V. Klyuchnikova and E. A. Lymar', "Production of metal composite materials," Glass and Ceramics, vol. 63, pp. 68-69, 2006.

[201] M. Kantha Babu and O. V. Krishnaiah Chetty, "A study on the use of single mesh size abrasives in abrasive waterjet machining," The International Journal of Advanced Manufacturing Technology, vol. 29, pp. 532-540, 2006.

[202] I. Karakurt, G. Aydin, and K. Aydiner, "An Experimental study on the depth of cut of granite in abrasive waterjet cutting," Materials and Manufacturing Processes, vol. 27, pp. 538-544, 2012/05/01 2012. 